\title{
A propriedade de Borsuk-Ulam para funções entre superfícies
}

\author{
Vinicius Casteluber Laass
}

TESE APRESENTADA

$\mathrm{AO}$

Instituto de Matemática e Estatística

DA

UNIVERSIDADE DE SÃO PAUlO

PARA

OBTENÇÃO DO TÍTULO

$\mathrm{DE}$

Doutor EM CiÊnCIAS

Programa: Matemática

Orientador: Prof. Dr. Daciberg Lima Gonçalves

Coorientador: Prof. Dr. John Guaschi

Durante o desenvolvimento deste trabalho o autor recebeu auxílio financeiro do CNPq, projeto 140836/2012-8, e

do programa de cooperação Capes/COFECUB, projeto 12693/13-8.

São Paulo, julho de 2015 


\section{A propriedade de Borsuk-Ulam para funções entre superfícies}

Esta versão da tese contém as correções e alterações sugeridas pela Comissão Julgadora durante a defesa da versão original do trabalho, realizada em 21/07/2015. Uma cópia da versão original está disponível no

Instituto de Matemática e Estatística da Universidade de São Paulo.

Comissão Julgadora:

- Prof. Dr. Daciberg Lima Gonçalves (orientador) - IME-USP

- Profa. Dra. Lucília Daruiz Borsari - IME-USP

- Prof. Dr. Oziride Manzoli Neto - ICMC-USP

- Prof. Dr. Daniel Vendrúscolo - UFSCAR

- Prof. Dr. Weslem Liberato Silva - UESC 
Para

Giovanna e Giulianna. 


\section{Agradecimentos}

Acho que eu sou um cara privilegiado. Digo isso, porque durante a minha formação acadêmica tive vários professores empolgados e amigos e isso por si só já seria um enorme privilégio. Estes professores sempre me diziam que para fazer um bom doutorado em matemática, muito mais do que capacidade ou instituição, era necessário ter um bom orientador. Bom, gostaria de dizer a esses meus mestres que eles tinham total razão e que sou um cara muito privilegiado, pois não tive um bom orientador, tive três.

À professora Lucília Borsari, minha orientadora acadêmica, gostaria de agradecer toda a atenção durante o período de disciplinas e também no decorrer do curso. Muito obrigado por aquele telefonema em que me empolgou e me convenceu a ir fazer o doutorado no IME - USP.

Aos professores Daciberg Gonçalves e John Guaschi, meus orientadores de tese, eu tenho que dizer uma coisa: Não sei quem escolheu o tema (ou se foram os dois), mas eu adorei. Tudo bem que teve meia dúzia de momentos em que eu me desesperei porque não sabia o que fazer com certas equações no grupo de tranças do Toro ou da garrafa de Klein, mas vocês cumpriram com seus papéis de orientadores e no resumo da ópera eu me diverti bastante. Muito obrigado a vocês dois pela paciência e por todos os ensinamentos, inclusive os matemáticos.

No final do curso me deu uma sensação que passou tudo num piscar de olhos, mas aí eu parei e pensei, caramba, foram quatro anos e meio. E certamente este tempo todo valeu a pena não apenas pelo crescimento acadêmico, mas porque este tempo foi feito de momentos com pessoas mais que especiais.

Logo no começo do curso, a então secretária da Comissão de Pós-Graduação alugou um quarto em sua casa para eu morar. De brinde eu ganhei uma família, com direito a duas cachorras festeiras e duas novas irmãs mais festeiras ainda (a quem dedico este trabalho). Muito obrigado Helena Oliveira, pelo carinho maternal que você teve comigo.

Também tive a oportunidade de estreitar os laços com meu tio Waldemar Kunsch e minha tia Margarida Kunsch, os quais eu gostaria de agradecer por todo o carinho. Vocês são para mim os exemplos de que a dedicação ao trabalho sempre vale a pena.

Se eu tive a oportunidade de chegar até aqui, grande mérito se deve ao meu pai, Sergio, e a minha mãe, Dora, pois os mesmos sempre me deram apoio incondicional. Mesmo morando a mil quilômetros de distância, ou até mesmo separados por um oceano, eu só tenho a agradecer por sempre estarem tão pertos. Ah, e ao meu irmão Diogo, valeu pelas figuras. E para que não role ciúme, ao meu irmão Gabriel, valeu pela camaradagem ou qualquer coisa genérica.

Bem, quando a família não estava tão perto, os amigos cumpriram seus papéis. Muito obrigado pelos vários momentos maravilhosos com todos vocês. Me desculpe quem eu não vou citar, mas seria injusto eu não falar explicitamente sobre quatro pessoas.

À minha amiga Travesti, que também é conhecida por Andreza Beezão e não é um travesti, não 
vou me esquecer dos seus SMS que chegavam às três e meia da madrugada. Muito obrigado por toda atenção na mudança de São Carlos para São Paulo.

Ao meu amigo Falcão, vulgo Rafael Souza, foram muito boas as nossas discussões acaloradas sobre recobrimentos, homologias, sequências espectrais... E muito obrigado por quase sempre elas terminarem com muita risada e com um copo gelado na mão.

À minha amiga Carolina Pereiro, que se chama Carolina mesmo, merci beaucoup por toda a amizade, principalmente no tempo em que fomos vizinhos em Caen. Jamais vou me esquecer das inúmeras quebradas de galho quando você era minha intérprete de francês.

Ao meu amigo Maikel Samuays, carinhosamente Mundissa, eu poderia escrever um livro sobre de como nos ajudamos mutuamente durante quatro anos e sobre todas as nossas aventuras em Sampa, mas resumindo, eu prefiro simplesmente dizer muito obrigado por existir e por fazer parte da minha vida. 


\section{Resumo}

LAASS, V. C. A propriedade de Borsuk-Ulam para funções entre superfícies. 2015. Tese (Doutorado) - Instituto de Matemática e Estatística, Universidade de São Paulo, São Paulo, 2015.

Sejam $M$ e $N$ superfícies fechadas e $\tau: M \rightarrow M$ uma involução livre de pontos fixos. Dizemos que uma classe de homotopia $\beta \in[M, N]$ tem a propriedade de Borsuk-Ulam se para toda função contínua $g: M \rightarrow N$ que representa $\beta$, existe $x \in M$ tal que $g(\tau(x))=g(x)$. No caso em que $N \neq \mathbb{S}^{2}, \mathbb{R P}^{2}$, mostramos que $\beta$ não ter a propriedade de Borsuk-Ulam é equivalente a existência de um diagrama algébrico envolvendo $\pi_{1}(M), \pi_{1}\left(M_{\tau}\right), P_{2}(N)$ e $B_{2}(N)$, sendo $M_{\tau}$ o espaço de órbitas de $\tau$ e sendo $P_{2}(N)$ e $B_{2}(N)$, respectivamente, o grupo de tranças puras e totais de $N$. Para cada caso listado abaixo, nós classificamos todas as classes de homotopia $\beta \in[M, N]$ que têm a propriedade de Borsuk-Ulam:

- $M=\mathbb{T}^{2}, M_{\tau}=\mathbb{T}^{2}$ e $N=\mathbb{T}^{2}$

- $M=\mathbb{K}^{2}$ e $N=\mathbb{K}^{2}$;

- $M=\mathbb{T}^{2}, M_{\tau}=\mathbb{K}^{2}$ e $N=\mathbb{T}^{2}$;

- $M=\mathbb{T}^{2}, M_{\tau}=\mathbb{T}^{2}$ e $N=\mathbb{K}^{2}$.

No caso em que $N=\mathbb{S}^{2}$, para cada superfície $\mathrm{M}$ e involução $\tau: M \rightarrow M$, nós classificamos os elementos $\beta \in\left[M ; \mathbb{S}^{2}\right]$ que têm a propriedade de Borsuk-Ulam. Para fazer tal classificação, nós usamos a teoria de funções equivariantes e a teoria de grau de aplicações. Para classes de homotopia $\beta \in\left[M ; \mathbb{R}^{2}\right]$, classificamos aquelas que se levantam para $\mathbb{S}^{2}$. No final, nós consideramos a propriedade de Borsuk-Ulam para ações livres de $\mathbb{Z}_{p}$, com $p$ um inteiro primo positivo. Neste caso, mostramos que se $M$ e $N$ são superfícies fechadas e $\mathbb{Z}_{p}$ age livremente em $\mathrm{M}$, com $p \neq 2$, então sempre existe uma função $f: M \rightarrow N$ homotópica a uma função constante e cuja restrição a cada órbita da ação é injetora.

Palavras-chave: Teorema de Borsuk-Ulam, superfícies, grupos de tranças. 


\section{Abstract}

LAASS, V. C. The Borsuk-Ulam property for functions between surfaces. 2015. Tese (Doutorado) - Instituto de Matemática e Estatística, Universidade de São Paulo, São Paulo, 2015.

Let $\mathrm{M}$ and $\mathrm{N}$ be compact surfaces without boundary, and let $\tau: M \rightarrow M$ be a fixed-point free involution. We say that a homotopy class $\beta \in[M, N]$ has the Borsuk-Ulam property if for every continuous function $g: M \rightarrow N$ that represents $\beta$, there exists $x \in M$ such that $g(\tau(x))=g(x)$. In the case where $N \neq \mathbb{S}^{2}, \mathbb{R P}^{2}$, we show that the fact that $\beta$ does not have the Borsuk-Ulam property is equivalent to the existence of an algebraic diagram involving $\pi_{1}(M), p i_{1}\left(M_{\tau}\right), P_{2}(N)$ and $B_{2}(N)$, where $M_{\tau}$ is the orbit space of $\tau$ and $P_{2}(N)$ and $B_{2}(N)$ are the pure and the full braid groups of the surface $N$ respectively. We then go on to consider the cases of the torus $\mathbb{T}^{2}$ and the Klein bottle $\mathbb{K}^{2}$. Let $M$ and $N$ satisfy one of the following:

- $M=\mathbb{T}^{2}, M_{\tau}=\mathbb{T}^{2}$ and $N=\mathbb{T}^{2}$

- $M=\mathbb{K}^{2}$ and $N=\mathbb{K}^{2}$;

- $M=\mathbb{T}^{2}, M_{\tau}=\mathbb{K}^{2}$ and $N=\mathbb{T}^{2}$;

- $M=\mathbb{T}^{2}, M_{\tau}=\mathbb{T}^{2}$ and $N=\mathbb{K}^{2}$.

In these cases we classify the homotopy classes $\beta \in[M, N]$ that possess the Borsuk-Ulam property. If $N=\mathbb{S}^{2}$, for every surface $M$ and an involution $\tau: M \rightarrow M$, we classify the elements $\beta \in\left[M, \mathbb{S}^{2}\right]$ that possess the Borsuk-Ulam property. To obtain this classification, we make use of the theory of equivariant functions and degree theory of maps. For homotopy classes $\beta \in\left[M, \mathbb{R P}^{2}\right]$, we classify the classes that admit a lifting to $\mathbb{S}^{2}$. Finally, we consider the Borsuk-Ulam property for free actions of $\mathbb{Z}_{p}$, where $p$ is a prime number. If $M$ and $N$ are compact surfaces without boundary such that $\mathbb{Z}_{p}$ acts freely on $M$, with $p \neq 2$, we show that there is always a function $f: M \rightarrow N$ homotopic to the constant function whose restriction to every orbit of $\tau$ is injective.

Keywords: Borsuk-Ulam Theorem, surfaces, braid groups. 


\section{Sumário}

$\begin{array}{ll}\text { Introdução } & 1\end{array}$

1 Preliminares e Generalidades $\quad 5$

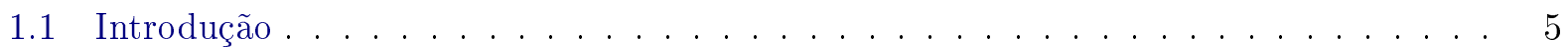

1.2 Classes de Homotopia . . . . . . . . . . . . . . . . . . 5

1.3 Classificação das involuções . . . . . . . . . . . . . . . . . . 6

1.4 A propriedade de Borsuk-Ulam e os Grupos de Tranças . . . . . . . . . . . . . . . . . . . .

1.5 Algumas notações sobre $\mathbb{T}^{2}$ e $\mathbb{K}^{2}$ e seus grupos fundamentais . . . . . . . . . . . . 16

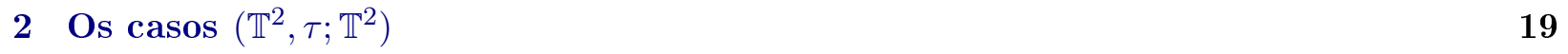

2.1 Introdução . . . . . . . . . . . . . . . . . . . . . . . . 19

2.2 Classificação das classes de homotopia com a propriedade de Borsuk-Ulam com res-

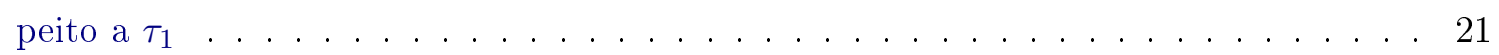

2.3 Classificação das classes de homotopia com a propriedade de Borsuk-Ulam com res-

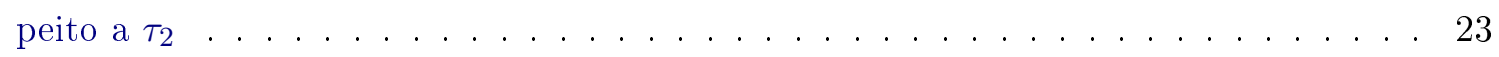

3 O caso $\left(\mathbb{K}^{2}, \tau ; \mathbb{K}^{2}\right) \quad \mathbf{3 1}$

3.1 Introdução . . . . . . . . . . . . . . . . . . . . . . 31

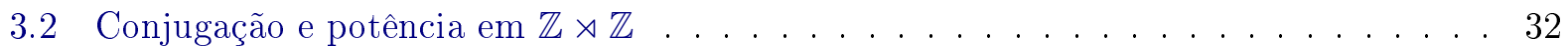

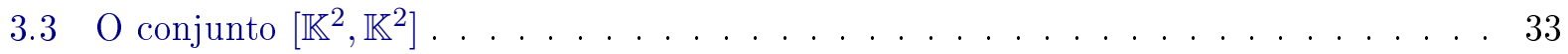

3.4 Classificação das classes de homotopia com a propriedade de Borsuk-Ulam . . . . . . 34

$4 \mathrm{O}$ caso $\left(\mathbb{T}^{2}, \tau ; \mathbb{K}^{2}\right)$, com $\mathbb{T}_{\tau}^{2}=\mathbb{T}^{2}$

4.1 Introdução . . . . . . . . . . . . . . . . . . . . 39

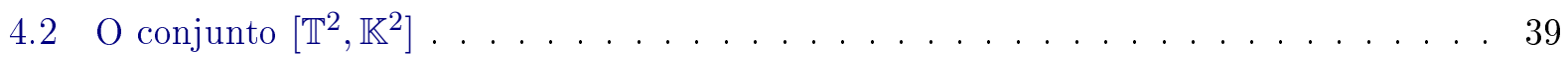

4.3 Algumas propriedades de $\overline{\left\langle\sigma^{2}\right\rangle} \ldots \ldots \ldots \ldots \ldots \ldots \ldots \ldots \ldots$

4.4 Classificação das classes de homotopia com a propriedade de Borsuk-Ulam . . . . . 53

4.4.1 Prova das Proposições 4.4.3, 4.4.4, 4.4.6, 4.4.7 e 4.4.9 . . . . . . . . . . . 55

4.4 .2 Prova da Proposição $4.4 .5 \ldots \ldots \ldots \ldots$. . . . . . . . . . . . . 59

4.4 .3 Prova das Proposições 4.4 .8 e $4.4 .10 \ldots \ldots$. . . . . . . . . . . . . 64

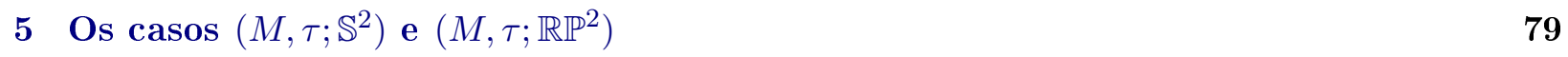

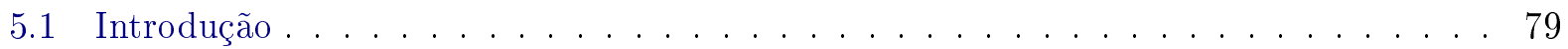

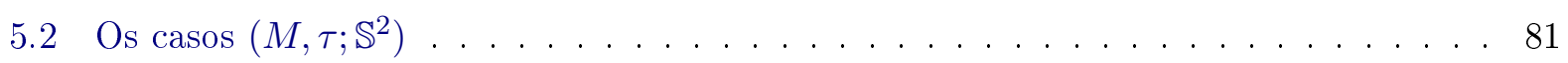

5.2 .1 Prova do Teorema $5.1 .2 \ldots \ldots \ldots \ldots \ldots \ldots$

5.2 .2 Prova do Teorema $5.1 .3 \ldots \ldots \ldots \ldots \ldots$ 
5.2 .3 Prova do Teorema $5.1 .4 \ldots \ldots \ldots \ldots$. . . . . . . . . . . 88

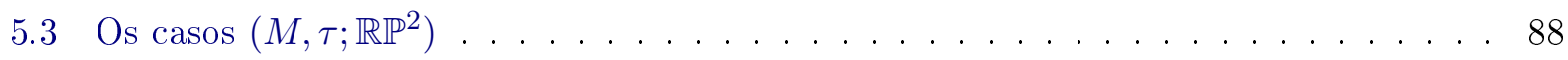

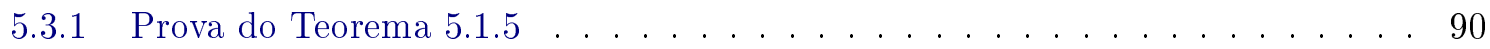

5.3 .2 Prova do Teorema $5.1 .6 \ldots \ldots \ldots \ldots \ldots$

6 A propriedade de Borsuk-Ulam para ações de $\mathbb{Z}_{p} \quad 93$

6.1 Introdução . . . . . . . . . . . . . . . . . . . . . . 93

6.2 Ações livre de $\mathbb{Z}_{p}$ e a propriedade de Borsuk-Ulam . . . . . . . . . . . . . . 93

$\begin{array}{ll}\text { A Os grupos de tranças do Toro } & 99\end{array}$

$\begin{array}{ll}\text { B Os grupos de tranças da garrafa de Klein } & 105\end{array}$

C O grupo fundamental dos espaços de configuração $\mathcal{A}_{2}\left(\mathbb{S}^{2}\right)$ e $\mathcal{B}_{2}\left(\mathbb{S}^{2}\right) \quad 125$

$\begin{array}{ll}\text { Referências Bibliográficas } & 127\end{array}$ 


\section{Introdução}

No início do século XX, o matemático polonês Stanislaw Ulam conjecturou que se $f: \mathbb{S}^{n} \rightarrow \mathbb{R}^{n}$ é uma aplicação contínua, então existe $x \in \mathbb{S}^{n}$ tal que $f(A(x))=f(x)$, sendo $A: \mathbb{S}^{n} \rightarrow \mathbb{S}^{n}$ a aplicação antipodal. Em 1933, o também matemático polonês Karol Borsuk confirmou este resultado em [5]. Este trabalho é o início da história do que hoje chamamos Teoremas do tipo Borsuk-Ulam. Mais detalhes sobre a história e as generalizações do Teorema de Borsuk-Ulam podem ser encontradas em [23].

Entre as muitas generalizações do problema proposto por Ulam e resolvido por Borsuk, a seguinte generalização é próxima do presente trabalho:

Definição 0.0.1. Sejam $M$ e $N$ espaços topológicos e $\tau: M \rightarrow M$ uma involução livre de pontos fixos, isto é, $\tau^{2}=I d_{M}$ e $\tau(x) \neq x$ para todo $x \in M$. Dizemos que a tripla $(M, \tau ; N)$ tem a propriedade de Borsuk-Ulam se para toda função contínua $f: M \rightarrow N$, existe $x \in M$ tal que $f(\tau(x))=f(x)$.

Vamos descrever alguns resultados que são próximos e relevantes para este trabalho.

Em 2006, Daciberg Lima Gonçalves classificou todas as triplas $\left(M, \tau ; \mathbb{R}^{2}\right)$ que têm a propriedade de Borsuk-Ulam nos casos em que $M$ é uma superfície fechada [15].

Em 2010, Daciberg Lima Gonçalves e John Guaschi classificaram todas as triplas $(M, \tau ; N)$ que têm a propriedade de Borsuk-Ulam nos casos em que $M$ e $N$ são superfícies fechadas [16].

Observemos que se $\left(M, \tau ; \mathbb{R}^{2}\right)$ não tem a propriedade de Borsuk-Ulam, por definição, existe uma aplicação contínua $f: M \rightarrow \mathbb{R}^{2}$ tal que $f(\tau(x)) \neq f(x)$ para todo $x \in M$. Como o conjunto das classes de homotopia de funções de $M$ em $\mathbb{R}^{2}$ tem cardinalidade 1 , isto é, o conjunto $\left[M, \mathbb{R}^{2}\right]$ tem apenas um elemento, vale que se $g: M \rightarrow \mathbb{R}^{2}$ é uma função contínua tal que $g(\tau(x))=g(x)$ para algum $x \in M$ (por exemplo, $g$ é uma função constante), então $g$ é homotópica a $f$, ou seja, $g$ é homotópica a uma função que não colapsa nenhuma órbita da involução $\tau$.

Agora, se $(M, \tau ; N)$ não tem a propriedade de Borsuk-Ulam, então existe uma função contínua $f: M \rightarrow N$ tal que $f(\tau(x)) \neq f(x)$ para todo $x \in M$. Mas se $g: M \rightarrow N$ é tal que $g(\tau(x))=g(x)$ para algum $x \in M$, não sabemos (a priori) se $g$ é homotópica a $f$ ou se $g$ é homotópica a alguma aplicação que não colapse conjuntos da forma $\{x, \tau(x)\}, x \in M$. Ou seja, no caso em que $(M, \tau ; N)$ não tem a propriedade de Borsuk-Ulam não temos uma boa informação de quais funções que a menos de homotopia não colapsam órbita da involução $\tau$.

A partir destas considerações, temos a formulação de um problema mais refinado, que motiva a seguinte definição, a qual pode ser considerada um refinamento da Definição 0.0.1.

Definição 0.0.2. Sejam $\tau: M \rightarrow M$ uma involução livre de pontos fixos e $\beta \in[M, N]$ uma classe de homotopia de funçôes entre $M$ e $N$. Dizemos que $\beta$ tem a propriedade de Borsuk-Ulam (com respeito a $\tau$ ) se para toda aplicação continua $g: M \rightarrow N$ que representa $\beta$, existe $x \in M$ tal que $g(\tau(x))=g(x)$.

Por negação, segue que se $\beta$ não tem a propriedade de Borsuk-Ulam, então existe uma função $f: M \rightarrow N$ que representa $\beta$ tal que $f(\tau(x)) \neq f(x)$ para todo $x \in M$.

Notemos que uma involução livre de pontos fixos $\tau: M \rightarrow M$ pode ser identificada como uma ação livre de $\mathbb{Z}_{2}$ em $M$. Uma outra forma de generalizar o Teorema de Borsuk-Ulam, que é bem 
conhecida e tem sido estudada, é substituirmos esta ação de $\mathbb{Z}_{2}$ por uma ação livre do grupo cíclico $\mathbb{Z}_{p}$, onde $p$ é um primo ímpar. O problema que se coloca, e que generaliza a questão clássica, é determinar se existe uma função contínua $f: M \rightarrow N$ tal que $f$ é injetora quando restrita a cada órbita da ação de $\mathbb{Z}_{p}$.

Neste trabalho de tese, a parte principal consiste em dar uma contribuição ao seguinte problema: para cada terna $(M, \tau ; N)$, sendo $M$ e $N$ superfícies fechadas e $\tau: M \rightarrow M$ uma involução livre de pontos fixos, classificar as classes de homotopia de funções de $M$ e $N$ que têm a propriedade de Borsuk-Ulam. De forma suscinta, neste trabalho classificaremos as classes de homotopia que têm a propriedade de Borsuk-Ulam para as ternas $(M, \tau ; N)$ listadas abaixo, sendo que $M_{\tau}$ denota o espaço de órbitas:

(1) $M=\mathbb{T}^{2}, M_{\tau}=\mathbb{T}^{2}$ e $N=\mathbb{T}^{2}$

(2) $M=\mathbb{T}^{2}, M_{\tau}=\mathbb{K}^{2}$ e $N=\mathbb{T}^{2}$;

(3) $M=\mathbb{K}^{2}$ e $N=\mathbb{K}^{2}$;

(4) $M=\mathbb{T}^{2}, M_{\tau}=\mathbb{T}^{2}$ e $N=\mathbb{K}^{2}$;

(5) $M$ sendo um superfície fechada orientável com $M_{\tau}$ orientável e $N=\mathbb{S}^{2}$;

(6) $M$ sendo um superfície fechada orientável com $M_{\tau}$ não orientável e $N=\mathbb{S}^{2}$;

(7) $M$ sendo um superfície fechada não orientável e $N=\mathbb{S}^{2}$.

De forma parcial, responderemos o problema de Borsuk-Ulam para as classes de homotopia de funções de $M$ em $\mathbb{R P}^{2}$ que se levantam para $\mathbb{S}^{2}$, onde $M$ poderá assumir todas as possibilidades de superfícies fechadas.

Relativo ao problema onde temos uma ação livre de $\mathbb{Z}_{p}$ em $M$, com $p$ primo e $p \neq 2$, no final deste trabalho, mostraremos que se $M$ e $N$ são superfícies fechadas, então sempre existe uma função contínua $f: M \rightarrow N$ homotópica a uma função constante e cuja restrição a cada órbita da ação é injetora.

Como etapa inicial para obtenção dos resultados acima, se faz necessário para cada dupla de superfícies fechadas $M$ e $N$ entender bem o conjunto das classes de homotopia de funções entre estes espaços. Também se faz necessário entender bem a classificação das involuções livres em $M$.

Relativo ao estudo das involuções livres em superfícies, os resultados são conhecidos e fizemos uma exposição dos mesmos na Seção 1.3, a qual é um resumo dos trabalhos [15, 16].

Já para se determinar o conjunto $[M, N]$, dependendo dos casos, as técnicas utilizadas são diferentes. Por isso, é conveniente a divisão em duas famílias distintas, que são as seguintes:

$$
\begin{aligned}
& \text { (a) } N \neq \mathbb{S}^{2}, \mathbb{R P}^{2} \text {; } \\
& \text { (b) } N=\mathbb{S}^{2} \text { ou } N=\mathbb{R P}^{2} \text {. }
\end{aligned}
$$

No caso (a), nós fixaremos, na Seção 1.2, a notação do fato bem conhecido de que o conjunto das classes de homotopia de funções entre $M$ e $N$ está em bijeção com as classes de conjugação dos homomorfismos de $\pi_{1}(M)$ e $\pi_{1}(N)$.

Usando este fato, na Seção 1.4, nós demonstraremos um critério algébrico para que um elemento $\beta \in[M, N]$ não tenha a propriedade de Borsuk-Ulam com respeito a uma involução $\tau: M \rightarrow M$ (Lema 1.4.3 e Teorema 1.4.4). Tal critério utiliza os grupos fundamentais de $M$ e do espaço de órbitas $M_{\tau}$ e também os grupos de tranças puras e totais de $N$, os quais denotamos por $P_{2}(N)$ e $B_{2}(N)$, respectivamente.

Este resultado generaliza a conexão iniciada em [16] de duas áreas importantes e ativas na matemática:

Teoremas do tipo Borsuk-Ulam $\longleftrightarrow$ Grupos de tranças 
Esta ligação de áreas se mostrou bastante frutífera para estudar o problema de Borsuk-Ulam para classes de homotopia.

Lembrando que os grupos de tranças foram introduzidas pelo matemático austríaco Emil Artin em 1925 [2]. Em 1962, os matemáticos americanos Ralph Hartzler Fox e Lee Neuwirth definiram os grupos de tranças de superfícies utilizando o grupo fundamental de um certo espaço construído a partir da superfície, chamado espaço de configuração, e mostrando que os grupos definidos por Artin eram um caso particular no caso em que a superfície considerada era o disco unitário $\mathbb{D}^{2}$ [14]. A formulação em termos do grupo fundamental é a que é conveniente para o nosso estudo.

Com os resultados teóricos estabelecidos, passamos a trabalhar em casos específicos.

No Capítulo 2, nós classificaremos os elementos do conjunto $\left[\mathbb{T}^{2}, \mathbb{T}^{2}\right]$ que têm a propriedade de Borsuk-Ulam com respeito a uma involução $\tau_{1}: \mathbb{T}^{2} \rightarrow \mathbb{T}^{2}$, cuja principal característica é que o espaço de órbitas $\mathbb{T}_{\tau_{1}}^{2}$ é homeomorfo a $\mathbb{T}^{2}$ e também classificaremos as classes de homotopia que têm a propriedade de Borsuk-Ulam com respeito a uma involução $\tau_{2}: \mathbb{T}^{2} \rightarrow \mathbb{T}^{2}$, cuja principal característica é que o espaço de órbitas $\mathbb{T}_{\tau_{2}}^{2}$ é homeomorfo a $\mathbb{K}^{2}$. No primeiro caso, nós mostraremos que se $\beta \in\left[\mathbb{T}^{2}, \mathbb{T}^{2}\right.$, então $\beta$ não tem a propriedade de Borsuk-Ulam com respeito a $\tau_{1}$ (Teorema 2.2.3). No segundo caso, a situação é mais complexa e mostraremos quando $\beta$ tem a propriedade de Borsuk-Ulam com respeito a $\tau_{2}$ em termos do homomorfismo induzido em nível de grupo fundamental por algum representante de $\beta$, sendo que a condição sobre o homomorfismo é dada de forma completamente explícita (Teorema 2.3.10).

Para tal estudo, nós utilizaremos uma certa presentação de $P_{2}\left(\mathbb{T}^{2}\right)$ e como um certo elemento $\sigma \in B_{2}\left(\mathbb{T}^{2}\right)$ que não está em $P_{2}\left(\mathbb{T}^{2}\right)$ age por conjugação nesta presentação. Este é o conteúdo do Apêndice A.

No Capítulo 3, nós classificaremos os elementos do conjunto $\left[\mathbb{K}^{2}, \mathbb{K}^{2}\right]$ que têm a propriedade de Borsuk-Ulam com respeito a uma involução $\tau: \mathbb{K}^{2} \rightarrow \mathbb{K}^{2}$ fixada. Nós mostraremos que uma classe de homotopia $\beta \in\left[\mathbb{K}^{2}, \mathbb{K}^{2}\right]$ tem a propriedade de Borsuk-Ulam se, e somente se, esta classe de homotopia se levanta para $\mathbb{T}^{2}$ (Teorema 3.4.5).

Para tal estudo, também foi necessário mostrar uma presentação de $P_{2}\left(\mathbb{K}^{2}\right)$ e como um certo elemento $\sigma \in B_{2}\left(\mathbb{K}^{2}\right)$ que não está em $P_{2}\left(\mathbb{K}^{2}\right)$ age por conjugação nesta presentação. Este é um dos principais objetivos do Apêndice B.

No Capítulo 4, nós classificaremos os elementos do conjunto $\left[\mathbb{T}^{2}, \mathbb{K}^{2}\right]$ que têm a propriedade de Borsuk-Ulam com respeito a mesma involução $\tau_{1}: \mathbb{T}^{2} \rightarrow \mathbb{T}^{2}$ que está definida no Capítulo 2, mostrando exatamente quando uma classe de homotopia $\beta$ tem a propriedade de Borsuk-Ulam com respeito a $\tau_{1}$ em termos do homomorfismo induzido em nível de grupo fundamental por algum representante de $\beta$ (Teorema 4.4.2). Este caso se mostrou o de maior complexidade para se obter a classificação entre aqueles do caso $(a)$.

Para tal estudo, é necessário obter uma presentação do subgrupo normal gerado pelo elemento $\sigma^{2} \in P_{2}\left(\mathbb{K}^{2}\right)$. No Apêndice B, nós mostraremos que tal subgrupo é um grupo livre de posto infinito e explicitaremos uma base, o que é de suma importância para os cálculos.

Sobre o estudo teórico do caso (b), onde $N=\mathbb{S}^{2}$ ou $N=\mathbb{R P}^{2}$, na introdução do Capítulo 5 e mais especialmente no Teorema 5.1.1, nós fixaremos a notação do fato bem conhecido que o conjunto $\left[M, \mathbb{S}^{2}\right]$ está em bijeção, através da noção de grau, com $\mathbb{Z}$ se $M$ é orientável ou está em bijeção com $\mathbb{Z}_{2}$ se $M$ é não orientável. Mostraremos que uma classe de homotopia $\beta \in\left[M\right.$, $\left.\mathbb{S}^{2}\right]$ não tem a propriedade de Borsuk-Ulam com respeito a uma involução $\tau: M \rightarrow M$ se, e somente se, $\beta$ tem um representante que é $(\tau, A)$ equivariante, sendo $A: \mathbb{S}^{2} \rightarrow \mathbb{S}^{2}$ a involução antipodal (Lema 5.2.1).

Usando tais fatos teóricos, para todas as possibilidades de $M$ e de uma involução $\tau: M \rightarrow M$, nós classificaremos as classes de homotopia $\beta \in\left[M, \mathbb{S}^{2}\right]$ que têm a propriedade de Borsuk-Ulam (Teoremas 5.1.2, 5.1.3 e 5.1.4).

Como consequência dos resultados sobre funções com contra-domínio $\mathbb{S}^{2}$, foi possível estudar uma família de funções com contra-domínio $\mathbb{R P}^{2}$. Mais precisamente, para todas as possibilidades de $M$ e de uma involução $\tau: M \rightarrow M$, consideramos os elementos $\beta \in\left[M, \mathbb{R} \mathbb{P}^{2}\right]$ que se levantam para $\mathbb{S}^{2}$ e nós mostraremos exatamente quais destes elementos têm a propriedade de Borsuk-Ulam 
com respeito a uma involução $\tau$ (Teoremas 5.1.5 e 5.1.6).

No caso em que $M$ é não orientável, para fazer tal classificação, é necessário obter uma presentação do espaço de configuração $\mathcal{A}_{2}\left(\mathbb{S}^{2}\right)$ e de órbitas $\mathcal{B}_{2}\left(\mathbb{S}^{2}\right)$. Tais espaços são uma generalização dos espaços de configuração de $\mathbb{S}^{2}$. A definição destes espaços, bem como outras informações e resultados obtidos sobre os mesmos, estão no Apêndice C.

Por fim, no Capítulo 6, nós generalizaremos a Definição 0.0.1 para ações livres de $\mathbb{Z}_{p}$ em $M$, sendo $p$ um número inteiro primo positivo (Definição 6.2.3). Nós mostraremos que se $p \neq 2$, então para toda ação livre de $\mathbb{Z}_{p}$ em $M$, existe um função $f: M \rightarrow N$ homotópica a uma função constante tal que $f$ é injetora em cada órbita da ação (Teorema 6.2.5). 


\section{Capítulo 1}

\section{Preliminares e Generalidades}

\subsection{Introdução}

Os principais objetivos deste capítulo são:

- Fixar os fatos e as notações sobre classes de homotopia de funções que tem como contradomínio espaços do tipo $\mathrm{K}(\pi, 1)$. Tais resultados serão utilizados em boa parte deste trabalho.

- Fazer um resumo dos resultados encontrados nos trabalhos [15, 16], os quais serão úteis para estudar a propriedade de Borsuk-Ulam para classes de homotopia de funções.

- Mostrar o Lema Fundamental 1.4.3, assim como o Teorema 1.4.4, que são resultados téoricos que nos permitiram estudar a propriedade de Borsuk-Ulam para classes de homotopia de funções entre superfícies através de um diagrama algébrico envolvendo grupos de tranças.

Os fatos teóricos que serão descritos neste capítulo, serão usados nos próximos capítulos para estudar funções tendo como domínio o Toro ou a Garrafa de Klein e como contra-domínio também o Toro ou a Garrafa de Klein. Por esse motivo, nós incluímos uma seção fixando a notação que utilizaremos sobre tais espaços.

Em todo este trabalho funções e aplicações serão sempre contínuas, sendo omitido este termo.

\subsection{Classes de Homotopia}

Sejam $X$ e $Y$ espaços topólogicos e $f, g: X \rightarrow Y$ duas funções. Dizemos que $f$ é homotópica a $g$, e escrevemos $f \simeq g$, se existe $H: X \times[0,1] \rightarrow Y$ tal que $H(x, 0)=f(x)$ e $H(x, 1)=g(x)$ para todo $x \in X$.

O conjunto das classes de equivalência por esta relação, também chamado de conjunto de classes de homotopia livre de funções entre $X$ e $Y$, é denotado por $[X, Y]$. Se $f: X \rightarrow Y$ é uma função, então o símbolo $[f]$ representa a classe de homotopia de $f$. Se $\beta=[f] \in[X, Y]$, dizemos que $f$ é um representante da classe de homotopia $\beta$.

Vamos fazer precisamente as definições análogas para funções pontuadas.

Dizemos que $f:\left(X, x_{1}\right) \rightarrow\left(Y, y_{1}\right)$ é uma função pontuada, se $f: X \rightarrow Y$ é uma função e $f\left(x_{1}\right)=y_{1}$.

Dadas $f, g:\left(X, x_{1}\right) \rightarrow\left(Y, y_{1}\right)$, dizemos que $f$ é homotópica a $g$ relativamente ao ponto $x_{1}$, e escrevemos $f \simeq g\left(\right.$ rel $\left.x_{1}\right)$, se existe $H: X \times[0,1] \rightarrow Y$ tal que $H(x, 0)=f(x)$ e $H(x, 1)=g(x)$ para todo $x \in X$ e $H\left(x_{1}, t\right)=y_{1}$ para todo $t \in[0,1]$.

O conjunto das classes de equivalência por esta relação, também chamado de conjunto de classes de homotopia pontuada de funções entre $X$ e $Y$, é denotado por $\left[X, x_{1} ; Y, y_{1}\right]$. Se $f:\left(X, x_{1}\right) \rightarrow\left(Y, y_{1}\right)$ é uma função pontuada, então o símbolo $[f]$ representa a classe de homotopia de $f$. Se $\alpha=[f]$, então dizemos que $f$ é um representante de $\alpha$. 
Sejam $f, g:\left(X, x_{1}\right) \rightarrow\left(Y, y_{1}\right)$ duas funções pontuadas e suponhamos que $f \simeq g$ (rel $\left.x_{1}\right)$. Lembremos da teoria de homotopia que se $f_{\#}, g_{\#}: \pi_{1}\left(X, x_{1}\right) \rightarrow \pi_{1}\left(Y, y_{1}\right)$ são os homomorfismos induzidos nos grupos fundamentais, então $f_{\#}=g_{\#}$. Portanto, dada uma classe de homotopia pontuada $\alpha \in\left[X, x_{1} ; Y, y_{1}\right]$, podemos associar um elemento de $\operatorname{Hom}\left(\pi_{1}\left(X, x_{1}\right), \pi_{1}\left(Y, y_{1}\right)\right)$ do seguinte modo: escolhemos um representante $f$ de $\alpha$ e associamos o homomorfismo induzido nos grupos fundamentais. Ou seja, a seguinte função é bem definida:

$$
\begin{aligned}
\Gamma_{X, Y}:\left[X, x_{1} ; Y, y_{1}\right] & \longrightarrow \operatorname{Hom}\left(\pi_{1}\left(X, x_{1}\right), \pi_{1}\left(Y, y_{1}\right)\right) \\
\alpha=[f] & \longmapsto \Gamma_{X, Y}(\alpha)=f_{\#} .
\end{aligned}
$$

Sejam novamente $f, g:\left(X, x_{1}\right) \rightarrow\left(Y, y_{1}\right)$ duas funções pontuadas e suponhamos que $f \simeq g\left(\right.$ rel $\left.x_{1}\right)$. É claro que omitindo os pontos bases, ou mais precisamente, considerando as funções $f, g: X \rightarrow Y$, então $f \simeq g$, isto é, se $f$ é homotópica a $g$ relativamente ao ponto base $x_{1}$, então $f$ é homotópica a $g$. Portanto, dada uma classe de homotopia pontuada $\alpha \in\left[X, x_{1} ; Y, y_{1}\right]$, podemos associar uma classe de homotopia livre $\beta \in[X, Y]$ do seguinte modo: escolhemos um representante $f$ de $\alpha$ e associamos a classe de homotopia livre $\beta$ tal que $f$ é um representante. Ou seja, a seguinte função é bem definida:

$$
\begin{aligned}
\Lambda_{X, Y}:\left[X, x_{1} ; Y, y_{1}\right] & \longrightarrow[X, Y] \\
\alpha=[f] & \longmapsto \Lambda_{X, Y}(\alpha)=\beta=[f] .
\end{aligned}
$$

Dados dois homomorfismos $h_{1}, h_{2} \in \operatorname{Hom}\left(\pi_{1}\left(X, x_{1}\right), \pi_{1}\left(Y, y_{1}\right)\right)$, dizemos que $h_{1}$ é equivalente a $h_{2}$, e escrevemos $h_{1} \sim h_{2}$, se existe $\omega \in \pi_{1}\left(Y, y_{1}\right)$ tal que $h_{1}(v)=\omega h_{2}(v) \omega^{-1}$ para todo $v \in \pi_{1}\left(X, x_{1}\right)$, ou seja, os homomorfismos $h_{1}$ e $h_{2}$ são conjugados por um elemento de $\pi_{1}\left(Y, y_{1}\right)$. É fácil ver que $\sim$ é uma relação de equivalência. Seja

$$
\Upsilon_{X, Y}: \operatorname{Hom}\left(\pi_{1}\left(X, x_{1}\right), \pi_{1}\left(Y, y_{1}\right)\right) \longrightarrow \frac{\operatorname{Hom}\left(\pi_{1}\left(X, x_{1}\right), \pi_{1}\left(Y, y_{1}\right)\right)}{\sim}
$$

a projeção natural de um homomorfismo na sua classe de equivalência (ou classe de conjugação).

Em [27, Capítulo V, Teorema 4.3], está enunciado o fato bem conhecido que se $X$ e $Y$ são variedades topológicas conexas, com $\pi_{i}(Y)$ trivial se $i \neq 1$, então $\Gamma_{X, Y}$ é uma bijeção e $\Lambda_{X, Y}$ é uma sobrejeção. É claro que $\Upsilon_{X, Y}$ é uma sobrejeção. Em [27, Capítulo V, Corolário 4.4], está enunciado o fato que existe uma bijeção

$$
\Delta_{X, Y}:[X, Y] \longrightarrow \frac{\operatorname{Hom}\left(\pi_{1}\left(X, x_{1}\right), \pi_{1}\left(Y, y_{1}\right)\right)}{\sim}
$$

tal que $\Delta_{X, Y} \circ \Lambda_{X, Y}=\Upsilon_{X, Y} \circ \Gamma_{X, Y}$.

Nós podemos resumir estes fatos no seguinte resultado, o qual será muito utilizado neste trabalho de tese:

Teorema 1.2.1. Se $X$ e $Y$ são variedades topológicas conexas, com $\pi_{i}\left(Y, y_{1}\right)$ trivial se $i \neq 1$, então o seguinte diagrama é comutativo, sendo que as flechas horizontais são bijeções e as flechas verticais são sobrejeçôes:

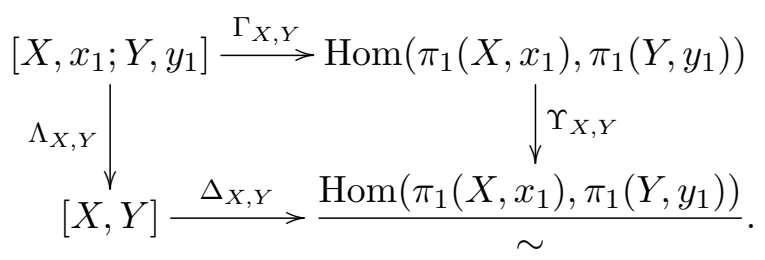

\subsection{Classificação das involuções}

Ao estudar a validade de teoremas do tipo Borsuk-Ulam, uma pergunta inicial que se faz é a seguinte: dado um espaço topológico $M$, "quantas" involuções existem em $M$ ? Mais ainda, se temos 
duas involuções $\tau_{1}, \tau_{2}: M \rightarrow M$ e sabemos a resposta para uma tripla $\left(M, \tau_{1} ; N\right)$, é possível a partir desta informação decidir se $\left(M, \tau_{2} ; N\right)$ tem a propriedade de Borsuk-Ulam ou não? Faremos aqui um resumo das respostas destas questões obtidas nos trabalhos [15, 16]. Posteriormente, vamos mostrar como estas informações nos ajudam a estudar o problema de Borsuk-Ulam para classes de homotopia.

Definição 1.3.1. Dizemos que duas involuções livres de pontos fixos $\tau_{1}, \tau_{2}: M \rightarrow M$ são equivalentes, se existe um homeomorfismo $H: M \rightarrow M$ tal que $H\left(\tau_{2}(x)\right)=\tau_{1}(H(x))$ para todo $x \in M$.

Teorema 1.3.2. Se $\tau_{1}, \tau_{2}: M \rightarrow M$ são equivalentes, então $\left(M, \tau_{1} ; N\right)$ não tem a propriedade de Borsuk-Ulam se, e somente se, $\left(M, \tau_{2} ; N\right)$ não tem a propriedade de Borsuk-Ulam.

Demonstração. Se $\left(M, \tau_{1} ; N\right)$ não tem a propriedade de Borsuk-Ulam, por definição, existe $f: M \rightarrow N$ tal que para todo $x \in M$ vale

$$
f\left(\tau_{1}(x)\right) \neq f(x)
$$

Assim, para todo $x \in M$ temos que

$$
(f \circ H)\left(\tau_{2}(x)\right)=f\left(H\left(\tau_{2}(x)\right)\right)=f\left(\tau_{1}(H(x)) \neq f(H(x))=(f \circ H)(x) .\right.
$$

Segue que $\left(M, \tau_{2} ; N\right)$ não tem a propriedade de Borsuk-Ulam. A outra implicação segue de maneira análoga, pois notemos $H \circ \tau_{2}=\tau_{1} \circ H \Rightarrow H^{-1} \circ \tau_{1}=\tau_{2} \circ H^{-1}$.

Notemos que a Definição 1.3.1 e o Teorema 1.3.2 nos fornecem uma resposta para a segunda questão. Vamos agora descrever um critério para dizer se duas involuções são equivalentes, e deste modo, obter uma resposta para a primeira questão.

Seja $\tau: M \rightarrow M$ uma involução livre de pontos fixos. Em $M$ definimos a seguinte relação:

$$
x \sim y \Leftrightarrow y \in\{x, \tau(x)\} .
$$

Usando o fato que $\tau^{2}=I d_{M}$, é fácil mostrar que $\sim$ é uma relação de equivalência. Denotamos por $M_{\tau}$ o espaço quociente (também chamado espaço de órbitas) e por $p_{\tau}: M \rightarrow M_{\tau}$ a projeção natural. Suponhamos que $M$ seja uma superfície fechada. Neste caso, $M_{\tau}$ também é uma superfície fechada e $p_{\tau}$ é um recobrimento duplo. Logo, temos a seguinte sequência exata

$$
1 \longrightarrow \pi_{1}\left(M, m_{1}\right) \stackrel{\left(p_{\tau}\right)_{\#}}{\longrightarrow} \pi_{1}\left(M_{\tau}, p_{\tau}\left(m_{1}\right)\right) \stackrel{\theta_{\tau}}{\longrightarrow} \mathbb{Z}_{2} \longrightarrow 1
$$

sendo que estamos identificando $\frac{\pi_{1}\left(M_{\tau}, p_{\tau}\left(m_{1}\right)\right)}{\left(p_{\tau}\right)_{\#}\left(\pi_{1}\left(M, m_{1}\right)\right)} \operatorname{com} \mathbb{Z}_{2}$ e $\theta_{\tau}$ é a projeção natural.

A partir de [15, Proposição 2.4], nós podemos enunciar o seguinte resultado:

Proposição 1.3.3. Sejam $M$ uma superfície fechada e $\tau_{1}, \tau_{2}: M \rightarrow M$ involuções livres. Então $\tau_{1}$ é equivalente a $\tau_{2}$ se, e somente se, existe um isomorfismo $\phi: \pi_{1}\left(M_{\tau_{1}}\right) \rightarrow \pi_{1}\left(M_{\tau_{2}}\right)$ tal que é comutativo o seguinte diagrama (omitimos os pontos bases):

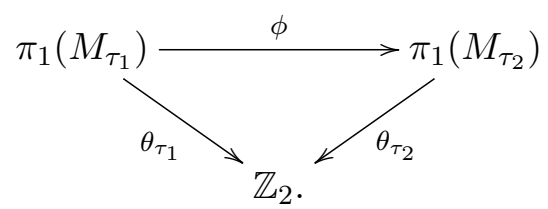

Observação 1.3.4. Pela Proposição acima e pelo Teorema de classificação de superfícies, segue que se $\tau_{1}$ e $\tau_{2}$ são equivalentes, então $M_{\tau_{1}}$ e $M_{\tau_{2}}$ são homeomorfos.

Em [16, Apêndice A], os autores usam o resultado da Proposição 1.3 .3 e fornecem um algoritmo efetivo para decidir quando duas involuções em uma superfície são equivalentes. A partir de [16, Proposição 30, Proposição 32], nós podemos enunciar o seguinte resultado de classificação: 
Teorema 1.3.5. Sejam $M$ uma superfície fechada e $\chi(M)$ a característica de Euler de $M$.

1. Se $M$ é orientável e $\frac{\chi(M)}{2}$ é par, então, a menos de equivalência, existem duas involuçôes $\tau_{1}, \tau_{2}: M \rightarrow M$. Se $M_{\tau_{1}}$ é orientável, então $M_{\tau_{2}}$ é não orientável.

2. Se $M$ é orientável e $\frac{\chi(M)}{2}$ é ímpar, então, a menos de equivalência, só existe uma involução $\tau: M \rightarrow M$ e o espaço de órbitas $M_{\tau}$ é não orientável.

3. Se $M=\mathbb{K}^{2}$, a menos de equivalência, só existe uma involução $\tau: \mathbb{K}^{2} \rightarrow \mathbb{K}^{2}$ e o espaço de órbitas $M_{\tau}$ é homeomorfo a $\mathbb{K}^{2}$.

4. Se $M$ é não orientável, $M \neq \mathbb{K}^{2}, \chi(M)$ é divisível por 2 e $\frac{\chi(M)}{2}$ é par, então, a menos de equivalência, existem duas involuções $\tau_{1}, \tau_{2}: M \rightarrow M$. Temos que

$$
\pi_{1}\left(M_{\tau_{1}}\right) \cong \pi_{1}\left(M_{\tau_{2}}\right) \cong\left\langle u, v, a_{1}, a_{2}, a_{2 n-1}, a_{2 n} \mid u v u v^{-1}\left[a_{1}, a_{2}\right] \ldots\left[a_{2 n-1}, a_{2 n}\right]\right\rangle
$$

sendo $n=-\frac{1}{2} \cdot \frac{\chi(M)}{2}$. Mais ainda, após aplicar o algoritmo fornecido em [16, Apêndice A], nós podemos supor, sem perda de generalidade, que os homomorfismos $\theta_{\tau_{1}}$ e $\theta_{\tau_{2}}$ são definidos, respectivamente, por:

$$
\theta_{\tau_{1}}:\left\{\begin{array}{l}
u \mapsto \overline{0} \\
v \mapsto \overline{0} \\
a_{1} \mapsto \overline{1} \\
a_{i} \mapsto \overline{0}, 1<i \leq 2 n
\end{array} \quad \theta_{\tau_{2}}:\left\{\begin{array}{l}
u \mapsto \overline{1} \\
v \mapsto \overline{0} \\
a_{i} \mapsto \overline{0}, 1 \leq i \leq 2 n .
\end{array}\right.\right.
$$

5. Se $M$ é não orientável, $M \neq \mathbb{K}^{2}, \chi(M)$ é divisivel por 2 e $\frac{\chi(M)}{2}$ é ímpar, então, a menos de equivalência, existem duas involuções $\tau_{1}, \tau_{2}: M \rightarrow M$. Temos que

$$
\pi_{1}\left(M_{\tau_{1}}\right) \cong \pi_{1}\left(M_{\tau_{2}}\right) \cong\left\langle c, a_{1}, a_{2}, a_{2 n-1}, a_{2 n} \mid c^{2}\left[a_{1}, a_{2}\right] \ldots\left[a_{2 n-1}, a_{2 n}\right]\right\rangle
$$

sendo $n=-\frac{1}{2}\left(\frac{\chi(M)}{2}-1\right)$. Mais ainda, após aplicar o algoritmo fornecido em [16, Apêndice A], nós podemos supor, sem perda de generalidade, que os homomorfismos $\theta_{\tau_{1}}$ e $\theta_{\tau_{2}}$ são definidos, respectivamente, por:

$$
\theta_{\tau_{1}}:\left\{\begin{array}{l}
c \mapsto \overline{0} \\
a_{1} \mapsto \overline{1} \\
a_{i} \mapsto \overline{0}, 1<i \leq 2 n
\end{array} \quad \theta_{\tau_{2}}:\left\{\begin{array}{l}
c \mapsto \overline{1} \\
a_{1} \mapsto \overline{1} \\
a_{i} \mapsto \overline{0}, 1<i \leq 2 n .
\end{array}\right.\right.
$$

6. Se $M$ é não orientável e $\chi(M)$ é ímpar, não é possível definir uma involução em $M$.

Nos dois seguintes teoremas nós reescrevemos os resultados [15, Teorema 2.5] e [16, Teorema 12]. Tais resultados nos fornecem uma classificação de triplas que têm a propriedade de Borsuk-Ulam, conforme a Definição 0.0.1.

Teorema 1.3.6. Sejam $M$ uma superfície fechada e $\tau: M \rightarrow M$ uma involução livre de pontos fixos. A tripla $\left(M, \tau ; \mathbb{R}^{2}\right)$ tem a propriedade de Borsuk-Ulam se, e somente se, ocorre uma das seguintes condiçôes: 
- $M$ é orientável e $\frac{\chi(M)}{2}$ é impar;

- $M=\mathbb{K}^{2}$;

- $M$ é não orientável, $M \neq \mathbb{K}^{2}, \chi(M)$ é par, $\frac{\chi(M)}{2}$ é par e, sem perda de generalidade, $\theta_{\tau}$ é igual ao homomorfismo $\theta_{\tau_{2}}$ descrito no item 4 do Teorema 1.3.5;

- $M$ é não orientável, $M \neq \mathbb{K}^{2}, \chi(M)$ é par, $\frac{\chi(M)}{2}$ é impar e, sem perda de generalidade, $\theta_{\tau}$ é igual ao homomorfismo $\theta_{\tau_{2}}$ descrito no item 5 do Teorema 1.3.5.

Teorema 1.3.7. Sejam $M$ e $N$ superfícies fechadas e $\tau: M \rightarrow M$ uma involução livre de pontos fixos. A tripla $(M, \tau ; N)$ tem a propriedade de Borsuk-Ulam se, e somente se, ocorre uma das seguintes condições:

- $M=\mathbb{S}^{2}$ e $N \neq \mathbb{S}^{2}$;

- $M=\mathbb{K}^{2}$ e $N$ é orientável e diferente de $\mathbb{S}^{2}$;

- $M=\mathbb{K}^{2} \# \mathbb{T}^{2}, \theta_{\tau}$ é, sem perda de generalidade, igual ao homomorfismo $\theta_{\tau_{2}}$ descrito no item 5 do Teorema 1.3 .5 e $N$ é orientável e diferente de $\mathbb{S}^{2}$.

Voltemos agora ao estudo do problema de Borsuk-Ulam para classes de homotopia.

Se $\left(M, \tau_{1} ; N\right)$ não tem a propriedade de Borsuk-Ulam, por definição, existe $f_{1}: M \rightarrow N$ tal que $f\left(\tau_{1}(x)\right) \neq f(x)$ para todo $x \in M$. Esta função $f_{1}$ representa alguma classe de homotopia $\beta_{1}$ e novamente, por definição, $\beta_{1}$ não tem a propriedade de Borsuk-Ulam com respeito a $\tau_{1}$. Se $\tau_{2}: M \rightarrow M$ é uma involução equivalente a $\tau_{1}$, e digamos que $H: M \rightarrow M$ é um homeomorfismo tal que $H \circ \tau_{2}=\tau_{1} \circ H$, então $f_{2}=f_{1} \circ H: M \rightarrow N$ representa um classe de homotopia $\beta_{2}$ que não tem a propriedade de Borsuk-Ulam com respeito a $\tau_{2}$. Não temos nenhuma garantia (a priori) que $\beta_{i}$ não tem a propriedade de Borsuk-Ulam com respeito a $\tau_{j}, i, j=1,2$ e $i \neq j$. Entretanto, com base nestas observações, nós podemos enunciar o seguinte resultado, o qual é de simples intuição, cuja demonstração não faremos, pois é, salvo alguns detalhes, igual a prova do Teorema 1.3.2.

Proposição 1.3.8. Sejam $\tau_{1}, \tau_{2}: M \rightarrow M$ duas involuções livres de pontos fixos e $H: M \rightarrow M$ um homeomorfismo tal que $H \circ \tau_{2}=\tau_{1} \circ H$. A seguinte funçâo é uma bijeção:

$$
\begin{aligned}
\mathcal{H}:[M, N] & \longrightarrow[M, N] \\
\beta=[f] & \longmapsto[f \circ H] .
\end{aligned}
$$

Mais ainda, uma classe de homotopia $\beta \in[M, N]$ não tem a propriedade de Borsuk-Ulam com respeito a $\tau_{1}$ se, e somente se, a classe de homotopia $\mathcal{H}(\beta)$ não tem a propriedade de Borsuk-Ulam com respeito a $\tau_{2}$.

Observação 1.3.9. Notemos que a Proposição 1.3 .8 nos diz que se $\tau_{1}, \tau_{2}: M \rightarrow M$ são duas involuçôes equivalentes, a quantidade de classes de homotopia que não têm a propriedade de BorsukUlam com respeito a $\tau_{1}$ é igual a quantidade de classes de homotopia que não têm a propriedade de Borsuk-Ulam com respeito a $\tau_{2}$. Por este motivo, sempre que formos estudar o problema de BorsukUlam para classes de homotopia, o faremos com base na classificação das involuções equivalentes dadas pelo Teorema 1.3.5.

\subsection{A propriedade de Borsuk-Ulam e os Grupos de Tranças}

Vamos obter nesta seção uma condição algébrica necessária e suficiente para que uma classe de homotopia tenha a propriedade de Borsuk-Ulam. Utilizaremos fortemente o Teorema 1.2.1 e sua notação, assim como a notação da Seção 1.3.

Para tanto, vamos primeiramente mostrar um resultado que nos dá uma completa caracterização algébrica para que uma classe de homotopia pontuada tenha um representante equivariante. 
Lema 1.4.1. Fixemos $M$ e $N$ variedades topólogicas conexas com $\pi_{i}\left(N, n_{1}\right)$ trivial se $i \neq 1$. Sejam $\tau: M \rightarrow M$ e $\tau_{1}: N \rightarrow N$ involuçôes livres de pontos fixos e $\alpha \in\left[M, m_{1} ; N, n_{1}\right]$ uma classe de homotopia pontuada. As seguintes condições são equivalentes:

1. $\alpha$ tem um representante $f:\left(M, m_{1}\right) \rightarrow\left(N, n_{1}\right)$ que é $\left(\tau, \tau_{1}\right)$-equivariante, isto é, para todo $x \in M$ vale a igualdade $f(\tau(x))=\tau_{1}(f(x))$;

2. existe um homomorfismo $\psi: \pi_{1}\left(M_{\tau}, p_{\tau}\left(m_{1}\right)\right) \rightarrow \pi_{1}\left(N_{\tau_{1}}, p_{\tau_{1}}\left(n_{1}\right)\right)$ tal que o seguinte diagrama é comutativo ( $\Gamma_{M, N}$ está definida na Seção 1.2):

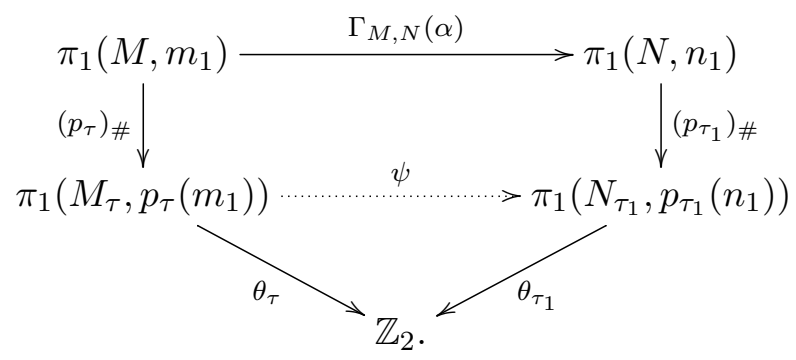

Mais ainda, valendo a condição 2 (e portanto a condição 1), então a não tem a propriedade de Borsuk-Ulam com respeito a $\tau$.

Demonstração. $(1 \Rightarrow 2)$ Suponhamos que a classe de homotopia pontuada $\alpha \in\left[M, m_{1} ; N, n_{1}\right]$ tem um representante $f:\left(M, m_{1}\right) \rightarrow\left(N, n_{1}\right)$ que é $\left(\tau, \tau_{1}\right)$-equivariante. Por este motivo, temos que para cada $x \in M$ vale $f\{x, \tau(x)\}=\left\{f(x), \tau_{1}(f(x))\right\}$. Logo, $f$ se passa ao quociente, isto é, existe $\bar{f}: M_{\tau} \rightarrow N_{\tau_{1}}$ tal que o seguinte diagrama é comutativo:

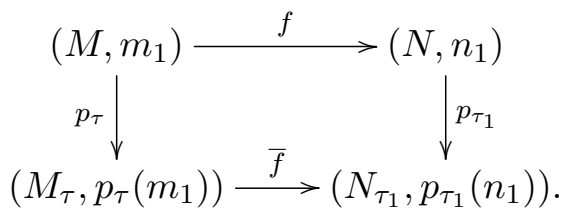

Tomamos $\psi=\bar{f}_{\#}$. Vamos mostrar que temos o diagrama comutativo (1.2). Primeiramente, temos que

$$
\psi \circ\left(p_{\tau}\right)_{\#}=\bar{f}_{\#} \circ\left(p_{\tau}\right)_{\#}=\left(\bar{f} \circ p_{\tau}\right)_{\#} \stackrel{(1.3)}{=}\left(p_{\tau_{1}} \circ f\right)_{\#}=\left(p_{\tau_{1}}\right)_{\#} \circ f_{\#}=\left(p_{\tau_{1}}\right)_{\#} \circ \Gamma_{M, N}(\alpha) .
$$

Seja agora $\alpha_{0} \in \pi_{1}\left(M_{\tau}, p_{\tau}\left(m_{1}\right)\right)$ tal que $\theta_{\tau}\left(\alpha_{0}\right)=\overline{0}$.

Então existe um elemento $\widetilde{\alpha_{0}} \in \pi_{1}\left(M, m_{1}\right)$ tal que $\left(p_{\tau}\right)_{\#}\left(\widetilde{\alpha_{0}}\right)=\alpha_{0}$.

Assim, temos que (o símbolo $*$ indica que estamos usando o fato que $\operatorname{Im}\left(p_{\tau_{1}}\right)_{\#}=\operatorname{ker} \theta_{\tau_{1}}$ )

$$
\left(\theta_{\tau_{1}} \circ \psi\right)\left(\alpha_{0}\right)=\left(\theta_{\tau_{1}} \circ \bar{f}_{\#} \circ\left(p_{\tau}\right)_{\#}\right)\left(\widetilde{\alpha_{0}}\right) \stackrel{(1.3)}{=}\left(\theta_{\tau_{1}} \circ\left(p_{\tau_{1}}\right)_{\#} \circ f_{\#}\right)\left(\widetilde{\alpha_{0}}\right) \stackrel{*}{=} \overline{0}=\theta_{\tau}\left(\alpha_{0}\right) .
$$

Por fim, seja $\alpha_{1} \in \pi_{1}\left(M_{\tau}, p_{\tau}\left(m_{1}\right)\right)$ tal que $\theta_{\tau}\left(\alpha_{1}\right)=\overline{1}$.

Seja $\gamma:([0,1],\{0,1\}) \rightarrow\left(M_{\tau}, p_{\tau}\left(m_{1}\right)\right)$ tal que $[\gamma]=\alpha_{1}$, sendo $[\gamma]$ a classe de homotopia do laço $\gamma$. Seja ainda $\xi:[0,1] \rightarrow M$ um caminho que é um levantamento do laço $\gamma$ pelo recobrimento $p_{\tau}$ tal que $\xi(0)=m_{1}$ e $\xi(1)=\tau\left(m_{1}\right)$. Como $f$ é equivariante, então $f \circ \xi:[0,1] \rightarrow N$ é tal que

$$
(f \circ \xi)(0)=f\left(m_{1}\right)=n_{1} \quad \text { e } \quad(f \circ \xi)(1)=f\left(\tau\left(m_{1}\right)\right)=\tau_{1}\left(f\left(m_{1}\right)\right)=\tau_{1}\left(n_{1}\right)
$$

e portanto é um caminho que não é um laço. Como $f \circ \xi$ é um levantamento do laço $\bar{f} \circ \gamma$ pelo recobrimento $p_{\tau_{1}}$, então a classe de homotopia de laço $[\bar{f} \circ \gamma]$ é um elemento de $\pi_{1}\left(N_{\tau_{1}}, p_{\tau_{1}}\left(n_{1}\right)\right)$ 
tal que $\theta_{\tau_{1}}[\bar{f} \circ \gamma]=\overline{1}$. Assim, temos que

$$
\left(\theta_{\tau_{1}} \circ \psi\right)\left(\alpha_{1}\right)=\left(\theta_{\tau_{1}} \circ \bar{f}_{\#}\right)[\gamma]=\theta_{\tau_{1}}[\bar{f} \circ \gamma]=\overline{1}=\theta_{\tau}\left(\alpha_{1}\right),
$$

o que completa o diagrama comutativo.

$(2 \Leftarrow 1)$ Suponhamos que temos o diagrama comutativo $(1.2)$ como no enunciado do Lema. Por hipótese, $N$ é uma variedade topólogica conexa tal que $\pi_{i}(N)$ é trivial se $i \neq 1$. Como $p_{\tau_{1}}$ é uma aplicação de recobrimento, então $N_{\tau_{1}}$ também é uma variedade topológica conexa tal que $\pi_{i}\left(N_{\tau_{1}}\right)$ é trivial se $i \neq 1$. Pelo Teorema 1.2.1, nós temos as bijeções $\Gamma_{M, N}$ e $\Gamma_{M_{\tau}, N_{\tau_{1}}}$. Logo, os homomorfismos $\Gamma_{M, N}(\alpha)$ e $\psi$ são induzidos por funções contínuas, ou seja, existem

(1) $g:\left(M, m_{1}\right) \rightarrow\left(N, n_{1}\right)$ tal que $\Gamma_{M, N}(\alpha)=g_{\#}$;

(2) $\bar{f}:\left(M_{\tau}, p_{\tau}\left(m_{1}\right)\right) \rightarrow\left(N_{\tau_{1}}, p_{\tau_{1}}\left(n_{1}\right)\right)$ tal que $\bar{f}_{\#}=\psi$.

Consideremos os seguintes elementos do conjunto $\left[M, m_{1} ; N_{\tau_{1}}, p_{\tau_{1}}\left(n_{1}\right)\right]$ :

$$
\alpha_{1}=\left[p_{\tau_{1}} \circ g\right] \quad \text { e } \alpha_{2}=\left[\bar{f} \circ p_{\tau}\right] .
$$

Notemos que temos a seguinte sequência de igualdades:

$$
\begin{gathered}
\Gamma_{M, N_{\tau_{1}}}\left(\alpha_{1}\right)=\left(p_{\tau_{1}} \circ g\right)_{\#}=\left(p_{\tau_{1}}\right)_{\#} \circ g_{\#} \stackrel{(1)}{=}\left(p_{\tau_{1}}\right)_{\#} \circ \Gamma_{M, N}(\alpha) \\
\stackrel{(1.2)}{=} \psi \circ\left(p_{\tau}\right)_{\#} \stackrel{(2)}{=} \bar{f}_{\#} \circ\left(p_{\tau}\right)_{\#}=\left(\bar{f} \circ p_{\tau}\right)_{\#}=\Gamma_{M, N_{\tau_{1}}}\left(\alpha_{2}\right)
\end{gathered}
$$

Novamente pelo Teorema 1.2.1, como $\Gamma_{M, N_{\tau_{1}}}$ é uma bijeção, então

$$
\left[p_{\tau_{1}} \circ g\right]=\alpha_{1}=\alpha_{2}=\left[\bar{f} \circ p_{\tau}\right]
$$

e portanto, $p_{\tau_{1}} \circ g \simeq \bar{f} \circ p_{\tau}\left(\right.$ rel $\left.m_{1}\right)$. Logo, existe uma homotopia $H: M \times[0,1] \rightarrow N_{\tau_{1}}$ tal que para todo $x \in M$ e para todo $t \in[0,1]$ valem:

(3) $H(x, 0)=\left(p_{\tau_{1}} \circ g\right)(x)$;

(4) $H(x, 1)=\left(\bar{f} \circ p_{\tau}\right)(x)$;

(5) $H\left(m_{1}, t\right)=p_{\tau_{1}}\left(n_{1}\right)$.

Por (3) e (5), segue que é comutativo o seguinte diagrama:

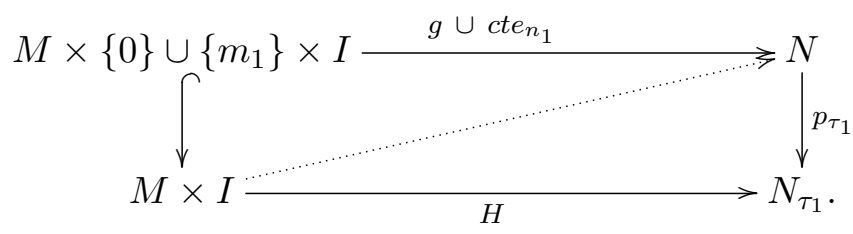

Usando [27, Capítulo II, Teorema 1.6 e Capítulo I, Teorema 7.16], podemos garantir que existe um levantamento da homotopia $H$, ou seja, existe $\widetilde{H}: M \times I \rightarrow N$ que completa o diagrama comutativo.

Definimos $f: M \rightarrow N$ por $f(x)=\widetilde{H}(x, 1)$. Pela comutatividade do diagrama anterior, temos que $f \simeq g$ rel $\left(m_{1}\right)$ e portanto $f_{\#}=g_{\#} \stackrel{(1)}{=} \Gamma_{M, N}(\alpha)$. Pelo Teorema 1.2.1, como $\Gamma_{M, N}$ é uma bijeção, então $f$ é um representante da classe de homotopia $\alpha$, isto é, $\alpha=[f]$. Vamos mostrar que $f$ é $\left(\tau, \tau_{1}\right)$-equivariante. Para todo $x \in M$ temos que:

$$
\left(p_{\tau_{1}} \circ f\right)(x)=p_{\tau_{1}}(\widetilde{H}(x, 1))=H(x, 1) \stackrel{(4)}{=}\left(\bar{f} \circ p_{\tau}\right)(x) .
$$


Assim, nós temos os seguintes diagramas comutativos:
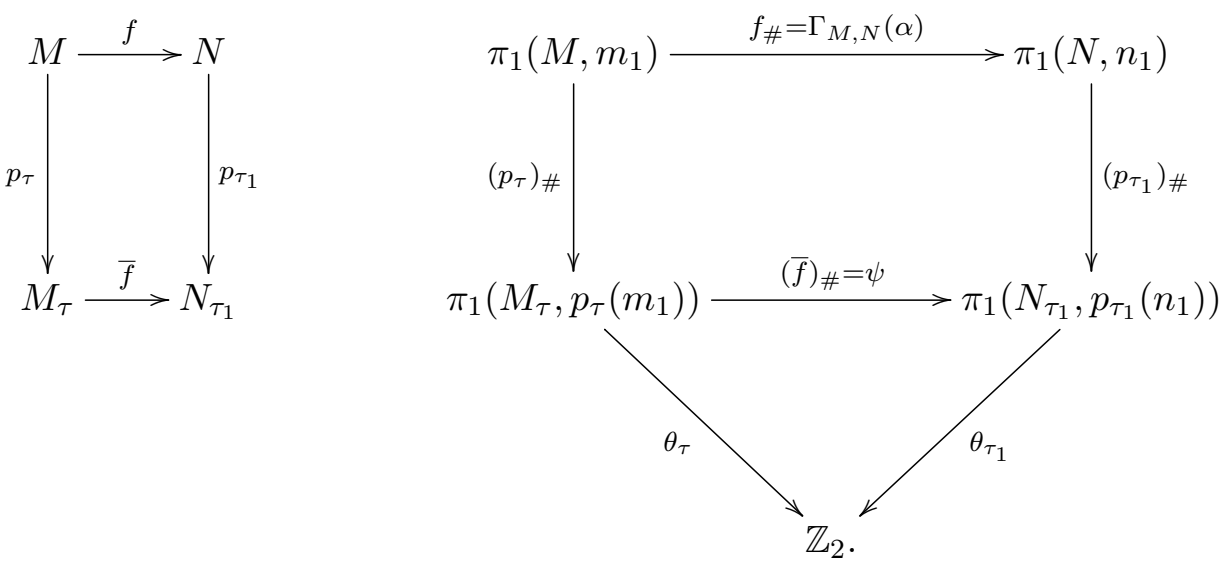

Do diagrama geométrico temos que para todo $x \in M$ vale

$$
p_{\tau_{1}}(f(\tau(x)))=\left(p_{\tau_{1}} \circ f\right)(\tau(x))=\left(\bar{f} \circ p_{\tau}\right)(\tau(x))=\left(\bar{f} \circ p_{\tau}\right)(x)=\left(p_{\tau_{1}} \circ f\right)(x)=p_{\tau_{1}}(f(x)) .
$$

Logo, ou $f(\tau(x))=f(x)$ ou $f(\tau(x))=\tau_{1}(f(x))$.

Seja $\xi:[0,1] \rightarrow M$ um caminho tal que $\xi(0)=m_{1}$ e $\xi(1)=\tau\left(m_{1}\right)$. Então o laço $\gamma=p_{\tau} \circ \xi$ é tal que a sua classe de homotopia $[\gamma]$ é mapeada por $\theta_{\tau}$ em $\overline{1}$. Pela comutatividade do diagrama algébrico temos que

$$
\theta_{\tau_{1}}[\bar{f} \circ \gamma]=\theta_{\tau_{1}} \circ \bar{f}_{\#}[\gamma]=\theta_{\tau}[\gamma]=\overline{1}
$$

e portanto, como $f \circ \xi$ é um levantamento do laço $\bar{f} \circ \gamma$ pelo recobrimento $p_{\tau_{1}}$, ele é um caminho que não é um laço. Assim, temos que

$$
f\left(m_{1}\right)=(f \circ \xi)(0) \neq(f \circ \xi)(1)=f\left(\tau\left(m_{1}\right)\right)
$$

e portanto vale

$$
f\left(\tau\left(m_{1}\right)\right)=\tau_{1}\left(f\left(m_{1}\right)\right) .
$$

Para mostrar que $f$ é equivariante, devemos mostrar que a igualdade acima é válida não somente para o ponto $m_{1}$, mas para todo os pontos de $M$. Seja então $x \in M$ e como $M$ é conexa, seja $\zeta:[0,1] \rightarrow M$ um caminho tal que

$$
\zeta(0)=m_{1} \quad \text { e } \quad \zeta(1)=x .
$$

Sejam $\delta_{1}, \delta_{2}:[0,1] \rightarrow N$ definidos, respectivamente, por $\delta_{1}=\tau_{1} \circ f \circ \zeta$ e $\delta_{2}=f \circ \tau \circ \zeta$. Temos que

$$
p_{\tau_{1}} \circ \delta_{1}=p_{\tau_{1}} \circ \tau_{1} \circ f \circ \zeta=p_{\tau_{1}} \circ f \circ \zeta=\bar{f} \circ p_{\tau} \circ \zeta=\bar{f} \circ p_{\tau} \circ \tau \circ \zeta=p_{\tau_{1}} \circ f \circ \tau \circ \zeta=p_{\tau_{1}} \circ \delta_{2}
$$

e

$$
\delta_{1}(0)=\left(\tau_{1} \circ f \circ \zeta\right)(0)=\tau_{1}\left(f\left(m_{1}\right)\right) \stackrel{(1.4)}{=} f\left(\tau\left(m_{1}\right)\right)=(f \circ \tau \circ \zeta)(0)=\delta_{2}(0),
$$

ou seja, $\delta_{1}$ e $\delta_{2}$ são levantamentos pelo recobrimento $p_{\tau_{1}}$ de um mesmo caminho (a saber $p_{\tau_{1}} \circ \delta_{1}$ ) e têm os mesmos pontos iniciais. Portanto, $\delta_{1}=\delta_{2}$. Assim, temos que

$$
\tau_{1}(f(x))=\left(\tau_{1} \circ f \circ \zeta\right)(1)=\delta_{1}(1)=\delta_{2}(1)=(f \circ \tau \circ \zeta)(1)=f(\tau(x)) .
$$

Por fim, notemos que por $f$ ser equivariante, para todo $x \in M$ vale

$$
f(\tau(x))=\tau_{1}(f(x)) \neq f(x),
$$


donde segue que $\alpha$ não tem a propriedade de Borsuk-Ulam com respeito a $\tau$.

Com o Lema 1.4.1, nós vamos usar grupos de tranças para estabelecer uma condição algébrica necessária e suficiente para que uma classe de homotopia pontuada tenha a propriedade de BorsukUlam.

Para tanto, vamos fixar alguns fatos e notações sobre espaços de configuração e grupos de tranças, os quais podem ser encontrados, por exemplo, em [4, 20, 24].

Em todo restante desta seção, $M$ e $N$ serão superfícies fechadas com $N \neq \mathbb{S}^{2}, \mathbb{R P}^{2}$ e $\tau: M \rightarrow M$ uma involução livre de pontos fixos.

A partir de $N$, definimos

$$
F_{2}(N)=\{(x, y) \in N \times N \mid x \neq y\},
$$

que é chamado de 2-espaço de configuração ordenado de $N$. Em $F_{2}(N)$ definimos a seguinte involução livre de pontos fixos:

$$
\begin{aligned}
\tau_{1}: F_{2}(N) & \longrightarrow F_{2}(N) \\
(x, y) & \longmapsto(y, x) .
\end{aligned}
$$

O espaço de órbitas por essa ação é denotado por $C_{2}(N)$, e é chamado de 2-espaço de configuração não ordenado de $N$. Denotamos por $p: F_{2}(N) \rightarrow C_{2}(N)$ a projeção natural.

Seja $n=\left(n_{1}, n_{2}\right) \in F_{2}(N)$. O grupo $\pi_{1}\left(F_{2}(N), n\right)$ é denotado por $P_{2}(N, n)$ e é chamado de grupo de 2-tranças puras de $N$. O grupo $\pi_{1}\left(C_{2}(N), p(n)\right)$ é denotado por $B_{2}(N, p(n))$ e é chamado de grupo de 2-tranças de $N$.

Em [13, Corolário 2.2], está demonstrado que $F_{2}(N)$ e $C_{2}(N)$ são variedades topológicas conexas tal que $\pi_{i}\left(F_{2}(N)\right)$ e $\pi_{i}\left(C_{2}(N)\right)$ são triviais se $i \neq 1$.

Analogamente a Sequência (1.1), Seção 1.3, nós temos a sequência exata

$$
1 \longrightarrow P_{2}(N, n) \stackrel{\iota}{\longrightarrow} B_{2}(N, p(n)) \stackrel{\pi}{\longrightarrow} \mathbb{Z}_{2} \longrightarrow 1,
$$

sendo $\iota=p_{\#}$ e $\pi$ definida de maneira natural.

Com essa notação definida e fixada, vamos começar a obter uma condição algébrica equivalente a propriedade de Borsuk-Ulam.

Notemos que na Definição 0.0 .2 , nós podemos trocar o conjunto $[M, N]$ por $\left[M, m_{1} ; N, n_{1}\right]$, ou seja, temos a seguinte definição:

Definição 1.4.2. Dizemos que uma classe de homotopia pontuada $\alpha \in\left[M, m_{1} ; N, n_{1}\right]$ tem a propriedade de Borsuk-Ulam com respeito a uma involução livre de pontos fixos $\tau: M \rightarrow M$, se para para toda função $g:\left(M, m_{1}\right) \rightarrow\left(N, n_{1}\right)$ que representa $\alpha$, existe $x \in M$ tal que $g(\tau(x))=g(x)$.

Suponhamos que $\alpha \in\left[M, m_{1} ; N, n_{1}\right]$ não tem a propriedade de Borsuk-Ulam com respeito a $\tau$. Por definição, existe $f:\left(M, m_{1}\right) \rightarrow\left(N, n_{1}\right)$ tal que $\alpha=[f]$ e $f(\tau(x)) \neq f(x)$ para todo $x \in M$. Então, está bem definida a seguinte função:

$$
\begin{aligned}
F: M & \longrightarrow F_{2}(N) \\
x & \longmapsto(f(x),(f \circ \tau)(x)) .
\end{aligned}
$$

Seja $p_{1}: F_{2}(N) \rightarrow N$ a projeção no primeiro fator, isto é, $p_{1}(x, y)=x$. Notemos que o seguinte diagrama é comutativo, sendo $n=\left(n_{1},(f \circ \tau)\left(n_{1}\right)\right)$

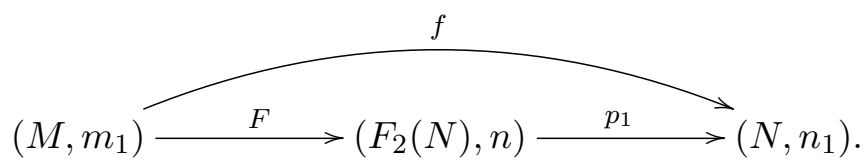


Notemos também que $F$ é $\left(\tau, \tau_{1}\right)$-equivariante. De fato, para todo $x \in M$ temos

$$
F(\tau(x))=(f(\tau(x)),(f \circ \tau)(\tau(x)))=((f \circ \tau)(x), f(x))=\tau_{1}(f(x),(f \circ \tau)(x))=\tau_{1}(F(x)) .
$$

Aplicando o Lema 1.4.1 para a classe de homotopia $[F] \in\left[M, m_{1} ; F_{2}(N), n\right]$ e o funtor grupo fundamental no diagrama (1.6), nós obtemos o seguinte diagrama comutativo:

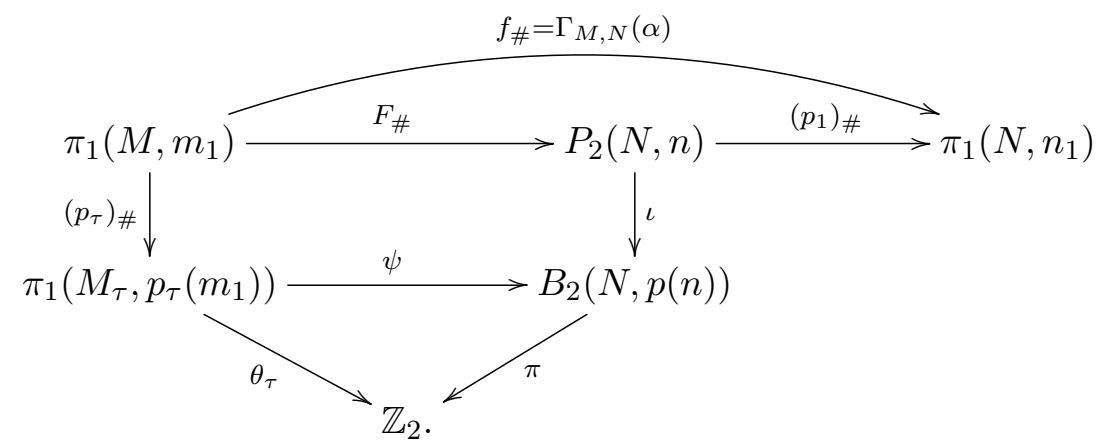

Portanto, nós mostramos que uma condição necessária para que $\alpha$ não tenha a propriedade de Borsuk-Ulam é a existência de um diagrama como acima. Tal condição também é suficiente. Mais precisamente, nós temos o seguinte resultado:

Lema 1.4.3 (O Lema Fundamental). Fixemos $M$ uma superfície fechada, $\tau: M \rightarrow M$ uma involução livre de pontos fixos e $N$ uma superfície fechada diferente de $\mathbb{S}^{2}$ e $\mathbb{R P}^{2}$. Seja $\alpha \in\left[M, m_{1} ; N, n_{1}\right]$ uma classe de homotopia pontuada. As seguintes condiçôes são equivalentes:

1. $\alpha$ não tem a propriedade de Borsuk-Ulam com respeito a $\tau$;

2. existem homomorfismos $\varphi: \pi_{1}\left(M, m_{1}\right) \rightarrow P_{2}(N, n)$ e $\psi: \pi_{1}\left(M_{\tau}, p_{\tau}\left(m_{1}\right)\right) \rightarrow B_{2}(N, p(n))$ que tornam o seguinte diagrama comutativo, sendo $\Gamma_{M, N}(\alpha)$ como definido na Seção $1.2 e$ $n=\left(n_{1}, n_{2}\right) \in F_{2}(N)$ para algum $n_{2} \in N$ :

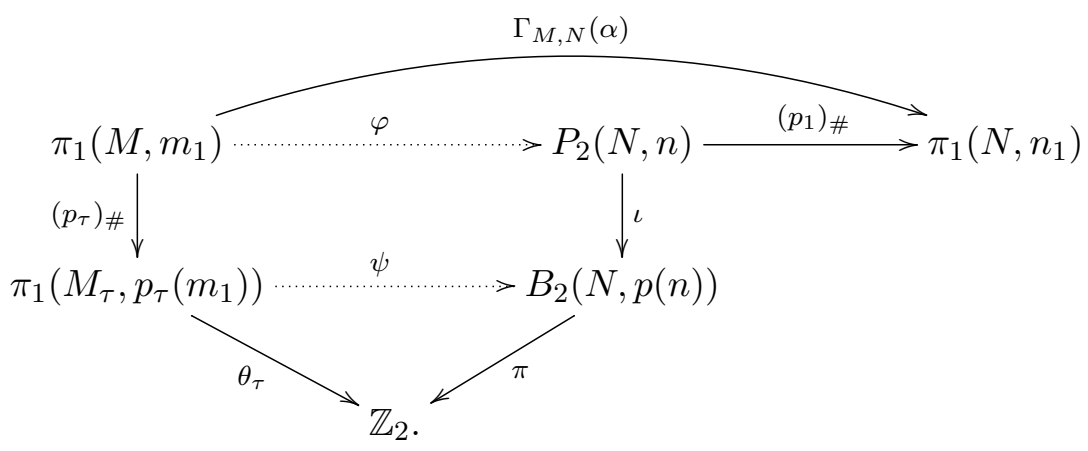

Demonstração. Já mostramos que $1 \Rightarrow 2$. Suponhamos então que seja verdadeira a condição 2 do enunciado do Lema, ou seja, temos o diagrama comutativo (1.7). Pelo Teorema 1.2.1, existe uma classe de homotopia pontuada

$$
\delta \in\left[M, m_{1} ; F_{2}(N), n\right] \text { tal que } \Gamma_{M, F_{2}(N)}(\delta)=\varphi .
$$

Pela comutatividade de (1.7), segue pelo Lema 1.4.1, que $\delta$ tem um representante

$$
F:\left(M, m_{1}\right) \rightarrow\left(F_{2}(N), n\right) \quad \text { tal que } \quad F(\tau(x))=\tau_{1}(F(x)) \text { para todo } \quad x \in M .
$$

Por definição de $\Gamma_{M, F_{2}(N)}$ (Seção 1.2), temos que $F_{\#}=\Gamma_{M, F_{2}(N)}(\delta)=\varphi$.

Seja $x \in M$. Temos que $F(x)=(f(x), g(x))$, sendo $f, g: M \rightarrow N$ duas funções tais que $f(a) \neq g(a)$ para todo $a \in M$. Logo 


$$
(f(\tau(x)), g(\tau(x)))=F(\tau(x))=\tau_{1}(F(x))=(g(x), f(x))
$$

e portanto, temos que $f(\tau(x))=g(x) \neq f(x)$.

Usando novamente a comutatividade do diagrama (1.7), temos as seguintes igualdades:

$$
\Gamma_{M, N}(\alpha)=\left(p_{1}\right)_{\#} \circ \varphi=\left(p_{1}\right)_{\#} \circ F_{\#}=\left(p_{1} \circ F\right)_{\#}=f_{\#} .
$$

Mais uma vez pelo Teorema 1.2.1, temos que $f$ é um representante da classe de homotopia $\alpha$, e portanto, $\alpha$ não tem a propriedade de Borsuk-Ulam.

Para finalizar esta seção, o próximo resultado nos mostrará que classificar classes de homotopia pontuada em relação a propriedade de Borsuk-Ulam é, em um certo sentido, o mesmo que classificar classes de homotopia livre em relação a propriedade de Borsuk-Ulam.

Teorema 1.4.4. Nas hipóteses do Lema Fundamental 1.4.3, sejam duas classes de homotopia pontuadas $\alpha, \alpha^{\prime} \in\left[M, m_{1} ; N, n_{1}\right]$ e uma classe de homotopia livre $\beta \in[M, N]$. Valem as seguintes condiçôes:

1. Se os homomorfismos $\Gamma_{M, N}(\alpha), \Gamma_{M, N}\left(\alpha^{\prime}\right): \pi_{1}\left(M, m_{1}\right) \rightarrow \pi_{1}\left(N, n_{1}\right)$ são conjugados (conforme a definição da Seção 1.2), então a não tem a propriedade de Borsuk-Ulam se, e somente se, $\alpha^{\prime}$ não tem a propriedade de Borsuk-Ulam.

2. Se $\beta=\Lambda_{M, N}(\alpha)$, então $\beta$ não tem a propriedade de Borsuk-Ulam se, e somente se, $\alpha$ não tem a propriedade de Borsuk-Ulam ( $\Lambda_{M, N}$ está definida na Seção 1.2).

Demonstração. 1. Suponhamos que $\alpha$ não tem a propriedade de Borsuk-Ulam. Então, pelo Lema Fundamental 1.4.3, existem homomorfismos

$$
\varphi: \pi_{1}\left(M, m_{1}\right) \rightarrow P_{2}(N, n) \quad \text { e } \quad \psi: \pi_{1}\left(M_{\tau}, p_{\tau}\left(m_{1}\right)\right) \rightarrow B_{2}(N, p(n))
$$

que juntamente com o homomorfismo $\Gamma_{M, N}(\alpha): \pi_{1}\left(M, m_{1}\right) \rightarrow \pi_{1}\left(N, n_{1}\right)$, satisfazem o diagrama $(1.7)$.

Como os homomorfismos $\Gamma_{M, N}(\alpha)$ e $\Gamma_{M, N}\left(\alpha^{\prime}\right)$ são conjugados, por definição, existe $\omega \in \pi_{1}\left(N, n_{1}\right)$ tal que $\Gamma_{M, N}\left(\alpha^{\prime}\right)(\gamma)=\omega\left(\Gamma_{M, N}(\alpha)(\gamma)\right) \omega^{-1}$ para todo $\gamma \in \pi_{1}\left(M, m_{1}\right)$.

Por [4, Teorema 1.4], o homomorfismo $\left(p_{1}\right)_{\#}: P_{2}(N, n) \rightarrow \pi_{1}\left(N, n_{1}\right)$ é sobrejetor. Logo, existe $b \in P_{2}(N, n)$ tal que $\left(p_{1}\right)_{\#}(b)=\omega$. Definimos:

$$
\begin{aligned}
\varphi^{\prime}: \pi_{1}\left(M, m_{1}\right) & \longrightarrow P_{2}(N, n) \\
\gamma & \longmapsto b(\varphi(\gamma)) b^{-1} \\
\psi^{\prime}: \pi_{1}\left(M_{\tau}, p_{\tau}\left(m_{1}\right)\right) & \longrightarrow B_{2}(N, p(n)) \\
\kappa & \longmapsto \iota(b)(\psi(\kappa)) \iota(b)^{-1} .
\end{aligned}
$$

e

Usando que $\varphi, \psi$ e $\Gamma_{M, N}(\alpha)$ satisfazem o diagrama (1.7) do Lema Fundamental 1.4.3, é fácil ver que os homomorfismos $\varphi^{\prime}, \psi^{\prime}$ e $\Gamma_{M, N}\left(\alpha^{\prime}\right)$ também satisfazem o diagrama (1.7) do mesmo Lema e portanto, $\alpha^{\prime}$ não tem a propriedade de Borsuk-Ulam.

Pela simetria do resultado, usando os mesmos argumentos, é claro que se $\alpha^{\prime}$ não tem a propriedade de Borsuk-Ulam, então o mesmo ocorre $\operatorname{com} \alpha$. 
2. Suponhamos que $\alpha$ não tem a propriedade de Borsuk-Ulam. Logo, existe $f:\left(M, m_{1}\right) \rightarrow\left(N, n_{1}\right)$ tal que $\alpha=[f]$ e $f(\tau(x)) \neq f(x)$ para todo $x \in M$. Como $\beta=\Lambda_{M, N}(\alpha)$, por definição de $\Lambda_{M, N}$, temos que $f: M \rightarrow N$ representa $\beta$, e portanto esta classe de homotopia livre não tem a propriedade de Borsuk-Ulam.

Reciprocamente, suponhamos que $\beta$ não tem a propriedade de Borsuk-Ulam. Então, existe uma função $f: M \rightarrow N$ tal que

$$
[f]=\beta \quad \text { e } \quad f(\tau(x)) \neq f(x) \quad \text { para todo } \quad x \in M .
$$

Como $N$ é uma superfície fechada, por [26, Lema 6.4] existe um homeomorfismo $H: N \rightarrow N$ tal que

$$
H \simeq \operatorname{Id}_{N} \quad \text { e } \quad H\left(f\left(m_{1}\right)\right)=n_{1} .
$$

Por construção, temos que a classe de homotopia pontuada $\alpha^{\prime}=[H \circ f] \in\left[M, m_{1} ; N, n_{1}\right]$ não tem a propriedade de Borsuk-Ulam. Usando a comutatividade do diagrama do Teorema 1.2.1, temos que

$$
\begin{aligned}
\left(\Upsilon_{M, N} \circ \Gamma_{M, N}\right)(\alpha) & =\left(\Delta_{M, N} \circ \Lambda_{M, N}\right)(\alpha)=\Delta_{M, N}(\beta)=\Delta_{M, N}([f]) \\
& =\Delta_{M, N}([H \circ f])=\left(\Delta_{M, N} \circ \Lambda_{M, N}\right)\left(\alpha^{\prime}\right)=\left(\Upsilon_{M, N} \circ \Gamma_{M, N}\right)\left(\alpha^{\prime}\right) .
\end{aligned}
$$

Pela definição de $\Upsilon_{M, N}$ (Seção 1.2), temos que os homomorfismos $\Gamma_{M, N}(\alpha)$ e $\Gamma_{M, N}\left(\alpha^{\prime}\right)$ são conjugados. Pelo item 1., segue que $\alpha$ não tem a propriedade de Borsuk-Ulam.

Observação 1.4.5. Notemos que a escolha do ponto $n_{2} \in N$ é irrelevante para decisão de existirem os homomorfismos que completam o diagrama (1.7) do Lema Fundamental 1.4.3, uma vez que $F_{2}(N)$ e $C_{2}(N)$ são variedades topológicas conexas. Na literatura em geral, o homomorfismo $\iota: P_{2}(N) \rightarrow B_{2}(N)$ é considerado como a inclusão e assim também faremos.

\subsection{Algumas notações sobre $\mathbb{T}^{2}$ e $\mathbb{K}^{2}$ e seus grupos fundamentais}

Nos próximos capítulos, nós teremos especial interesse em estudar a propriedade de BorsukUlam para classes de homotopia entre funções do Toro no Toro, da garrafa de Klein na garrafa de Klein e do Toro na garrafa de Klein. Por este motivo, nesta seção vamos fixar a notação que usaremos sobre estas superfícies e seus grupos fundamentais.

Consideremos a ação $\zeta_{1}:(\mathbb{Z} \oplus \mathbb{Z}) \times \mathbb{R}^{2} \longrightarrow \mathbb{R}^{2}$ definida nos geradores por

$$
\begin{aligned}
& \zeta_{1}(1,0)(x, y)=(x+1, y) \\
& \zeta_{1}(0,1)(x, y)=(x, y+1) .
\end{aligned}
$$

O espaço quociente por esta ação é o Toro, o qual denotamos por $\mathbb{T}^{2}$. Como $\mathbb{R}^{2}$ é o recobrimento universal do Toro, segue que, a menos de isomorfismo, podemos identificar $\pi_{1}\left(\mathbb{T}^{2}\right)$ com $\mathbb{Z} \oplus \mathbb{Z}$. A Figura 1.1 nos dá uma descrição geométrica de $\mathbb{T}^{2}$ como o quadrado $[0,1] \times[0,1]$ com arestas identificadas e do isomorfismo entre $\pi_{1}\left(\mathbb{T}^{2}\right)$ e $\mathbb{Z} \oplus \mathbb{Z}$.

Um ponto em $\mathbb{T}^{2}$ será denotado, fazendo um abuso de notação, simplesmente por um par ordenado $(x, y)$. Portanto, a igualdade $\left(x_{1}, y_{1}\right)=\left(x_{2}, y_{2}\right)$ em $\mathbb{T}^{2}$, significa que existe algum $(m, n) \in \mathbb{Z} \oplus \mathbb{Z}$ tal que $\zeta_{1}(m, n)\left(x_{1}, y_{1}\right)=\left(x_{2}, y_{2}\right)$.

Lembremos que se $G$ é um grupo e $h: \mathbb{Z} \oplus \mathbb{Z} \rightarrow G$ é uma função, então $h$ é um homomorfismo se, e somente se, $[h(1,0), h(0,1)]=1$, sendo $[a, b]=a b a^{-1} b^{-1}$ o comutador dos elementos $a$ e $b$ de $G$.

Agora consideremos a ação $\zeta_{2}:(\mathbb{Z} \rtimes \mathbb{Z}) \times \mathbb{R}^{2} \longrightarrow \mathbb{R}^{2}$ definida nos geradores por

$$
\begin{gathered}
\zeta_{2}(1,0)(x, y)=(x, y+1) \\
\zeta_{2}(0,1)(x, y)=(x+1,1-y) .
\end{gathered}
$$


O espaço quociente por esta ação é a garrafa de Klein, o qual denotamos por $\mathbb{K}^{2}$. Como $\mathbb{R}^{2}$ é o recobrimento universal da garrafa de Klein, segue que, a menos de isomorfismo, podemos identificar $\pi_{1}\left(\mathbb{K}^{2}\right)$ com $\mathbb{Z} \rtimes \mathbb{Z}$. A Figura 1.2 nos dá uma descrição geométrica de $\mathbb{K}^{2}$ como o quadrado $[0,1] \times[0,1]$ com arestas identificadas e do isomorfismo entre $\pi_{1}\left(\mathbb{K}^{2}\right)$ e $\mathbb{Z} \rtimes \mathbb{Z}$.

Um ponto em $\mathbb{K}^{2}$ será denotado, fazendo um abuso de notação, simplesmente por um par ordenado $(u, v)$. Portanto, a igualdade $\left(u_{1}, v_{1}\right)=\left(u_{2}, v_{2}\right)$ em $\mathbb{K}^{2}$, significa que existe algum $(r, s) \in \mathbb{Z} \rtimes \mathbb{Z}$ tal que $\zeta_{2}(r, s)\left(u_{1}, v_{1}\right)=\left(u_{2}, v_{2}\right)$.

Lembremos que se $G$ é um grupo e $h: \mathbb{Z} \rtimes \mathbb{Z} \rightarrow G$ é uma função, então $h$ é um homomorfismo se, e somente se, $[h(1,0), h(0,1)]^{\prime}=1$, sendo $[a, b]^{\prime}=a b a b^{-1}$ o anticomutador dos elementos $a$ e $b$ de $G$.

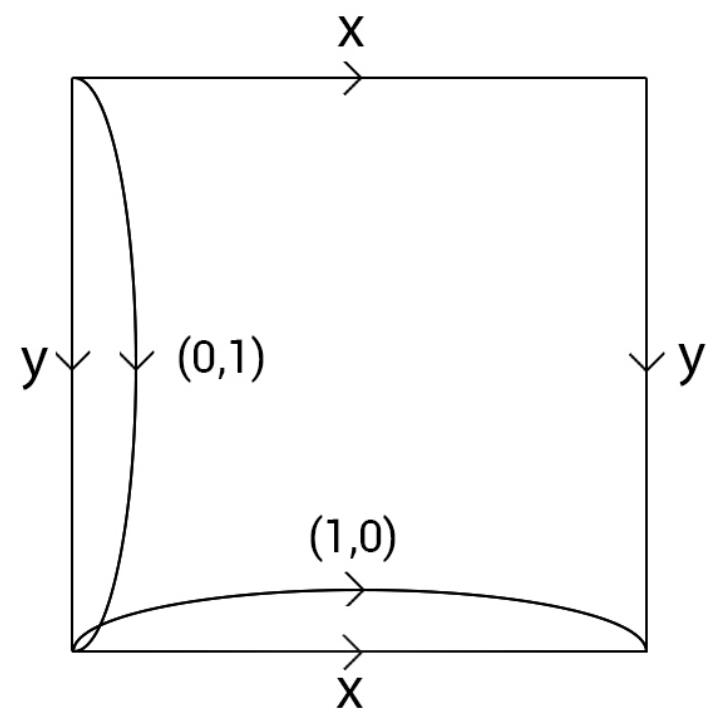

Figura 1.1: Toro.

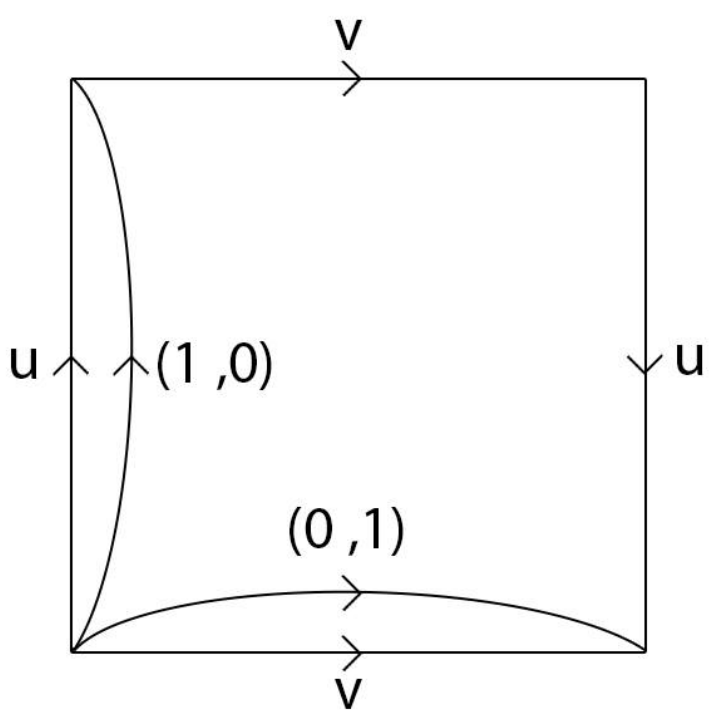

Figura 1.2: garrafa de Klein. 


\section{Capítulo 2}

\section{Os $\operatorname{casos}\left(\mathbb{T}^{2}, \tau ; \mathbb{T}^{2}\right)$}

\subsection{Introdução}

O objetivo deste capítulo é estudar a propriedade de Borsuk-Ulam para classes de homotopia de funções com domínio e contra-domínio sendo o Toro.

Segundo o Teorema 1.3.5, a menos de equivalência, existem duas involuções livres de pontos fixos em $\mathbb{T}^{2}$. Consideremos então as seguintes involuções:

$$
\begin{aligned}
& \tau_{1}: \mathbb{T}^{2} \longrightarrow \mathbb{T}^{2} \\
& (x, y) \longmapsto\left(x+\frac{1}{2}, y\right) \\
& \tau_{2}: \mathbb{T}^{2} \longrightarrow \mathbb{T}^{2} \\
& (x, y) \longmapsto\left(1-x,-y+\frac{1}{2}\right) .
\end{aligned}
$$

Temos que o espaço de órbitas $\mathbb{T}_{\tau_{1}}^{2}$ é homeomorfo a $\mathbb{T}^{2}$ e o espaço de órbitas $\mathbb{T}_{\tau_{2}}^{2}$ é homeomorfo a $\mathbb{K}^{2}$. As figuras 2.1 e 2.2 nos mostram como são as aplicações de recobrimento $p_{\tau_{1}}: \mathbb{T}^{2} \rightarrow \mathbb{T}^{2} \mathrm{e}$ $p_{\tau_{2}}: \mathbb{T}^{2} \rightarrow \mathbb{K}^{2}$

Utilizando a notação da Seção 1.5, podemos deduzir facilmente que os homomorfismos induzidos pelos recobrimentos $p_{\tau_{1}}$ e $p_{\tau_{2}}$ são definidos dos seguintes modos:

$$
\begin{aligned}
& \left(p_{\tau_{1}}\right)_{\#}: \quad \pi_{1}\left(\mathbb{T}^{2}\right)=\mathbb{Z} \oplus \mathbb{Z} \longrightarrow \mathbb{Z} \oplus \mathbb{Z}=\pi_{1}\left(\mathbb{T}^{2}\right) \quad \theta_{\tau_{1}}: \pi_{1}\left(\mathbb{T}^{2}\right)=\mathbb{Z} \oplus \mathbb{Z} \quad \longrightarrow \mathbb{Z}_{2} \\
& (1,0) \quad \longmapsto \quad(2,0) \quad(1,0) \quad \longmapsto \quad \overline{1} \\
& (0,1) \quad \longmapsto \quad(0,1) \quad(0,1) \quad \longmapsto \overline{0} \\
& \left(p_{\tau_{2}}\right)_{\#}: \quad \pi_{1}\left(\mathbb{T}^{2}\right)=\mathbb{Z} \oplus \mathbb{Z} \longrightarrow \mathbb{Z} \rtimes \mathbb{Z}=\pi_{1}\left(\mathbb{K}^{2}\right) \quad \theta_{\tau_{2}}: \quad \pi_{1}\left(\mathbb{K}^{2}\right)=\mathbb{Z} \rtimes \mathbb{Z} \longrightarrow \mathbb{Z}_{2} \\
& \begin{array}{lllll}
(1,0) & \longmapsto & (1,0) & (1,0) & \longmapsto \overline{0} \\
(0,1) & \longmapsto & (0,2) & (0,1) & \longmapsto \overline{1} .
\end{array}
\end{aligned}
$$

Observação 2.1.1. Utilizando as definições e notações estabelecidas na Seção 1.2, como $\pi_{1}\left(\mathbb{T}^{2}\right)=\mathbb{Z} \oplus \mathbb{Z}$ é um grupo abeliano, pelo Teorema 1.2.1 nós temos as seguintes bijeções:

$$
\begin{gathered}
\Gamma_{\mathbb{T}^{2}, \mathbb{T}^{2}}:\left[\mathbb{T}^{2}, * ; \mathbb{T}^{2}, *\right] \longrightarrow \operatorname{Hom}(\mathbb{Z} \oplus \mathbb{Z}, \mathbb{Z} \oplus \mathbb{Z}) \\
\Lambda_{\mathbb{T}^{2}, \mathbb{T}^{2}}:\left[\mathbb{T}^{2}, * ; \mathbb{T}^{2}, *\right] \longrightarrow\left[\mathbb{T}^{2}, \mathbb{T}^{2}\right] \quad \Delta_{\mathbb{T}^{2}, \mathbb{T}^{2}}:\left[\mathbb{T}^{2}, \mathbb{T}^{2}\right] \longrightarrow \operatorname{Hom}(\mathbb{Z} \oplus \mathbb{Z}, \mathbb{Z} \oplus \mathbb{Z}),
\end{gathered}
$$

as quais satisfazem $\Gamma_{\mathbb{T}^{2}, \mathbb{T}^{2}}=\Delta_{\mathbb{T}^{2}, \mathbb{T}^{2}} \circ \Lambda_{\mathbb{T}^{2}, \mathbb{T}^{2}}$. Para simplificar a notação, neste capítulo denotaremos $\Gamma_{\mathbb{T}^{2}, \mathbb{T}^{2}}, \Lambda_{\mathbb{T}^{2}, \mathbb{T}^{2}}$ e $\Delta_{\mathbb{T}^{2}, \mathbb{T}^{2}}$ apenas por $\Gamma, \Lambda$ e $\Delta$, respectivamente.

Sobre o grupo $P_{2}\left(\mathbb{T}^{2}\right)$, nós usaremos nas próximas seções os resultados estabelecidos no Apêndice A, em especial, a Observação A.0.10 a qual transcrevemos aqui. 


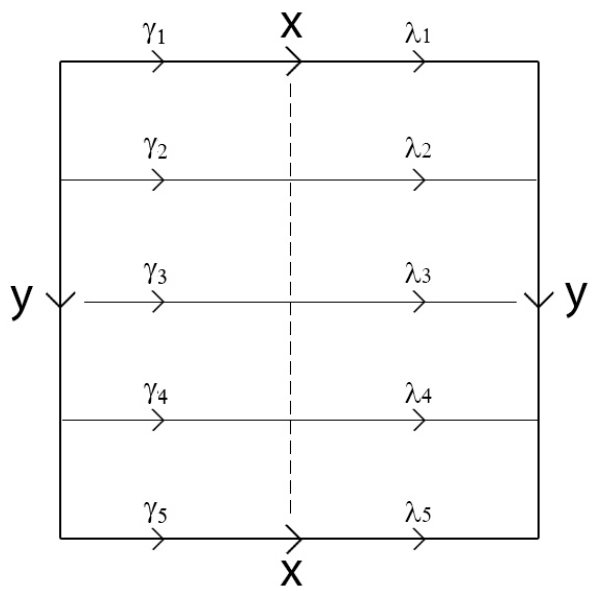

$p_{\tau_{1}}$

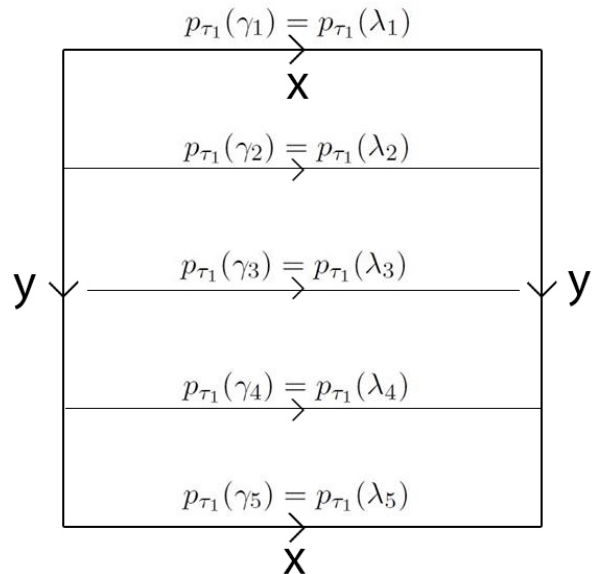

Figura 2.1: recobrimento $p_{\tau_{1}}$.
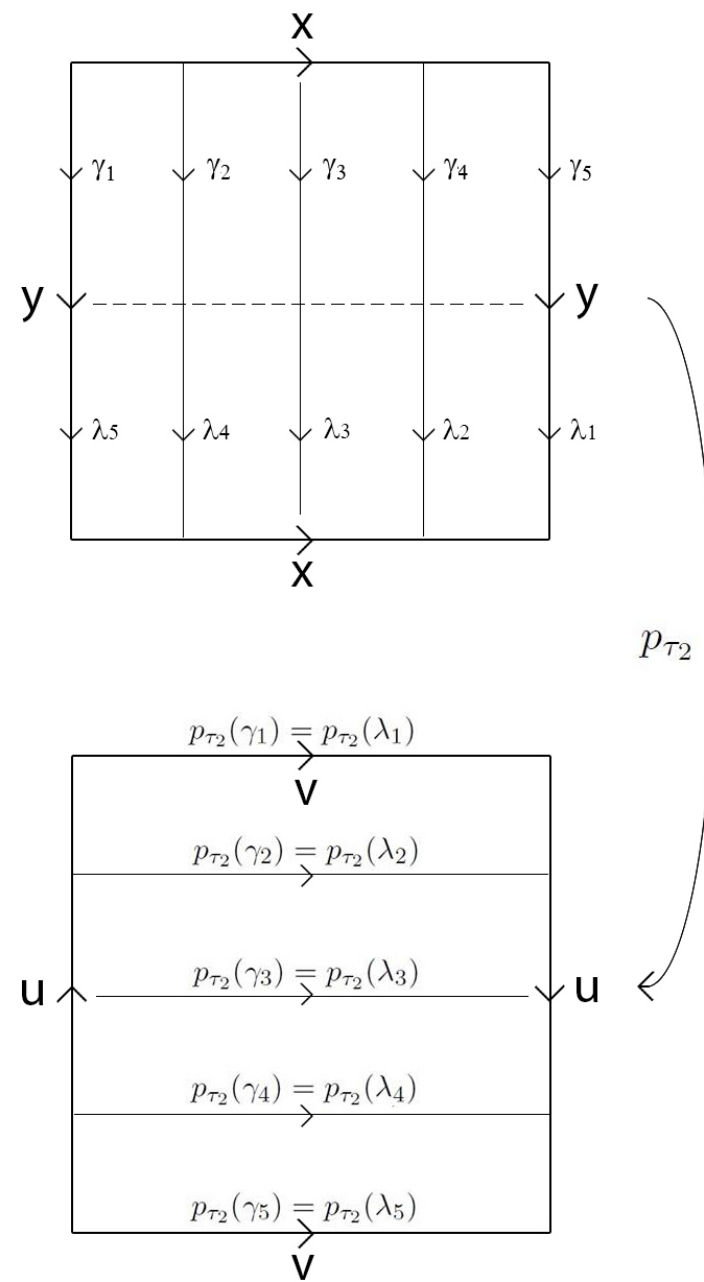

Figura 2.2: recobrimento $p_{\tau_{2}}$.

Observação 2.1.2 (Presentação de $P_{2}\left(\mathbb{T}^{2}\right)$ ). A menos de isomorfismo, $P_{2}\left(\mathbb{T}^{2}\right)$ se escreve na forma $F(x, y) \oplus \mathbb{Z} \oplus \mathbb{Z}$, sendo $F(x, y)$ o grupo livre gerado pelo conjunto $\{x, y\}$. Com respeito a esta escrita, temos que:

- $\left(p_{1}\right)_{\#}: P_{2}\left(\mathbb{T}^{2}\right) \rightarrow \pi_{1}\left(\mathbb{T}^{2}\right)=\mathbb{Z} \oplus \mathbb{Z}$ é a projeção de $F(x, y) \oplus \mathbb{Z} \oplus \mathbb{Z}$ em $\mathbb{Z} \oplus \mathbb{Z}$, isto é, $\left(p_{1}\right)_{\#}(w, m, n)=(m, n)$;

- Existe um elemento $\sigma \in B_{2}\left(\mathbb{T}^{2}\right)-P_{2}\left(\mathbb{T}^{2}\right)$ tal que $\sigma^{2}=(B, 0,0)$, sendo $B=\left[x, y^{-1}\right]$;

- O homomorfismo $l_{\sigma}: P_{2}\left(\mathbb{T}^{2}\right) \rightarrow P_{2}\left(\mathbb{T}^{2}\right)$, definido por $l_{\sigma}(b)=\sigma b \sigma^{-1}$ para todo $b \in P_{2}\left(\mathbb{T}^{2}\right)$, tem os seguintes valores nos geradores:

$$
\begin{array}{ll}
l_{\sigma}(x, 0,0)=\left(B x^{-1}, 1,0\right) & l_{\sigma}(\mathbb{1}, 1,0)=(\mathbb{1}, 1,0) \\
l_{\sigma}(y, 0,0)=\left(B y^{-1}, 0,1\right) & l_{\sigma}(\mathbb{1}, 0,1)=(\mathbb{1}, 0,1)
\end{array}
$$

sendo $\mathbb{1} \in F(x, y)$ o elemento neutro.

Observação 2.1.3. Se $t \in B_{2}\left(\mathbb{T}^{2}\right)-P_{2}\left(\mathbb{T}^{2}\right)$, ou seja, $t$ é uma trança não pura, então $t=a \sigma$, sendo $a=t \sigma^{-1} \in P_{2}\left(\mathbb{T}^{2}\right)$. Notemos que tal escrita é única, pois se $a_{1} \sigma=a_{2} \sigma$, então $a_{1}=a_{2}$.

Na Seção 2.2, nós mostraremos que toda classe de homotopia $\beta \in\left[\mathbb{T}^{2}, \mathbb{T}^{2}\right]$ não tem a propriedade de Borsuk-Ulam com relação a involução $\tau_{1}$ (Teorema 2.2.3). 
Com respeito a involução $\tau_{2}$, nós concluíremos na Seção 2.3 que existem elementos do conjunto $\left[\mathbb{T}^{2}, \mathbb{T}^{2}\right]$ que têm a propriedade de Borsuk-Ulam. Mais ainda, nós mostraremos exatamente quais são as classes de homotopia $\beta \in\left[\mathbb{T}^{2}, \mathbb{T}^{2}\right]$ que têm tal propriedade em termos do homomorfismo $\Delta(\beta)$ (Teorema 2.3.10).

\subsection{Classificação das classes de homotopia com a propriedade de Borsuk-Ulam com respeito a $\tau_{1}$}

Nesta seção, nós vamos classificar os elementos do conjunto $\left[\mathbb{T}^{2}, \mathbb{T}^{2}\right]$ em que vale a propriedade de Borsuk-Ulam com respeito a involução $\tau_{1}$. Para tal objetivo, o roteiro será utilizar o Lema Fundamental 1.4.3 para provar um critério algébrico equivalente a uma classe de homotopia pontuada não ter a propriedade de Borsuk-Ulam, aplicar tal critério e depois usar o Teorema 1.4.4 e a Observação 2.1.1. Em todas as demonstrações usaremos fortemente a Observação 2.1.2.

Proposição 2.2.1. Seja $\alpha \in\left[\mathbb{T}^{2}, * ; \mathbb{T}^{2}, *\right]$ uma classe de homotopia pontuada e seguindo a notação da Observação 2.1.1, seja $h_{\alpha}=\Gamma(\alpha): \pi_{1}\left(\mathbb{T}^{2}\right) \rightarrow \pi_{1}\left(\mathbb{T}^{2}\right)$. Então $\alpha$ não tem a propriedade de Borsuk-Ulam com respeito a $\tau_{1}$ se, e somente se, existem tranças puras $a, b \in P_{2}\left(\mathbb{T}^{2}\right)$ tais que:

(i) $a l_{\sigma}(b)=b a ;$

(ii) $h_{\alpha}(1,0)=\left(p_{1}\right)_{\#}\left(a l_{\sigma}(a)\right)$;

(iii) $h_{\alpha}(0,1)=\left(p_{1}\right)_{\#}(b)$.

Demonstração. Seja $\alpha$ uma classe de homotopia pontuada que não tem a propriedade de BorsukUlam e vamos mostrar que existem tranças $a, b \in P_{2}\left(\mathbb{T}^{2}\right)$ tais que são verdadeiras as condições $(i)$, (ii) e (iii). Pelo Lema Fundamental 1.4.3, existe o seguinte diagrama comutativo:

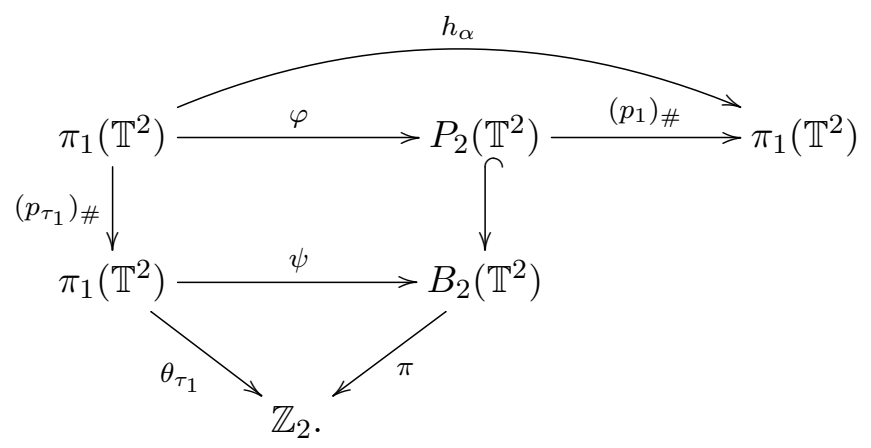

Por $(2.1)$, como $\theta_{\tau_{1}}(1,0)=\overline{1}$ e $\theta_{\tau_{1}}(0,1)=\overline{0}$, então pela comutatividade do diagrama $(2.3), \psi(1,0)$ deve ser uma trança não pura e $\psi(0,1)$ deve ser uma trança pura. Logo, pela Observação 2.1.3, existem tranças puras $a, b \in P_{2}\left(\mathbb{T}^{2}\right)$ tais que:

(1) $\psi(1,0)=a \sigma$

(2) $\psi(0,1)=b$.

Como $\pi_{1}\left(\mathbb{T}^{2}\right)$ é abeliano, então $1=[\psi(1,0), \psi(0,1)]=[a \sigma, b]=a \sigma b \sigma^{-1} a^{-1} b^{-1}=a l_{\sigma}(b) a^{-1} b^{-1}$. Portanto, vale

(i) $a l_{\sigma}(b)=b a$.

Novamente por $(2.1)$, como $\left(p_{\tau_{1}}\right)_{\#}(1,0)=(2,0)$ e $\left(p_{\tau_{1}}\right)_{\#}(0,1)=(0,1)$, segue da comutatividade de (2.3) que valem as seguintes igualdades para o homomorfismo $\varphi$ :

(3) $\varphi(1,0)=\left(\psi \circ\left(p_{\tau_{1}}\right)_{\#}\right)(1,0)=\psi(2,0)=\psi(1,0)^{2}=(a \sigma)^{2}=a \sigma a \sigma^{-1} \sigma^{2}=a l_{\sigma}(a) \sigma^{2}$;

(4) $\varphi(0,1)=\left(\psi \circ\left(p_{\tau_{1}}\right)_{\#}\right)(0,1)=\psi(0,1)=b$.

Novamente por (2.3) e pela Observação 2.1.2, valem as seguintes igualdades para o homomorfismo $h_{\alpha}:$ 
(ii) $h_{\alpha}(1,0)=\left(\left(p_{1}\right)_{\# \circ} \circ\right)(1,0)=\left(p_{1}\right)_{\#}\left(a l_{\sigma}(a) \sigma^{2}\right)=\left(p_{1}\right)_{\#}\left(a l_{\sigma}(a)\right)+\left(p_{1}\right)_{\#}(B, 0,0)=\left(p_{1}\right)_{\#}\left(a l_{\sigma}(a)\right)$;

(iii) $h_{\alpha}(0,1)=\left(\left(p_{1}\right)_{\#} \circ \varphi\right)(b)=\left(p_{1}\right)_{\#}(b)$.

Reciprocamente, suponhamos que existam tranças $a, b \in P_{2}\left(\mathbb{T}^{2}\right)$ e valem $(i)$, (ii) e (iii). Utilizando a Observação 2.1.3, definimos

$\mathrm{e}$

$$
\begin{aligned}
\varphi: \pi_{1}\left(\mathbb{T}^{2}\right) & \longrightarrow P_{2}\left(\mathbb{T}^{2}\right) \\
(1,0) & \longmapsto a l_{\sigma}(a) \sigma^{2} \\
(0,1) & \longmapsto b
\end{aligned}
$$

$$
\begin{aligned}
\psi: \pi_{1}\left(\mathbb{T}^{2}\right) & \longrightarrow B_{2}\left(\mathbb{T}^{2}\right) \\
(1,0) & \longmapsto a \sigma \\
(0,1) & \longmapsto b .
\end{aligned}
$$

Mostremos que de fato $\varphi$ e $\psi$ definem homomorfismos. Pela Observação 2.1.2, temos que

$$
\begin{aligned}
{[\varphi(1,0), \varphi(0,1)] } & =\left[a l_{\sigma}(a) \sigma^{2}, b\right]=a \sigma a \sigma^{-1} \sigma^{2} b \sigma^{-2} \sigma a^{-1} \sigma^{-1} a^{-1} b^{-1} \\
& =a l_{\sigma}\left(a l_{\sigma}(b) a^{-1}\right) a^{-1} b^{-1} \stackrel{(i)}{=} a l_{\sigma}\left(b a a^{-1}\right) a^{-1} b^{-1} \stackrel{(i)}{=} b a a^{-1} b^{-1}=1
\end{aligned}
$$

e

$$
[\psi(1,0), \psi(0,1)]=[a \sigma, b]=a \sigma b \sigma^{-1} a^{-1} b^{-1}=a l_{\sigma}(b) a^{-1} b^{-1} \stackrel{(i)}{=} b a a^{-1} b^{-1}=1 .
$$

Vamos mostrar que temos um diagrama comutativo como em (2.3), donde seguirá pelo Lema Fundamental 1.4.3 que $\alpha$ não tem a propriedade de Borsuk-Ulam. Utilizaremos as Observações 2.1.2 e 2.1.3 e também os valores dos homomorfismos $\left(p_{\tau_{1}}\right)_{\#}$ e $\theta_{\tau_{1}}$, os quais estão definidos em (2.1). Temos

$$
\begin{aligned}
& \left(\left(p_{1}\right)_{\#} \circ \varphi\right)(1,0)=\left(p_{1}\right)_{\#}\left(a l_{\sigma}(a) \sigma^{2}\right)=\left(p_{1}\right)_{\#}\left(a l_{\sigma}(a)\right)+\left(p_{1}\right)_{\#}(B, 0,0) \stackrel{(i i)}{=} h_{\alpha}(1,0), \\
& \left(\left(p_{1}\right)_{\#} \circ \varphi\right)(0,1)=\left(p_{1}\right)_{\#}(b) \stackrel{(i i i)}{=} h_{\alpha}(0,1),
\end{aligned}
$$

$$
\begin{aligned}
& \left(\psi \circ\left(p_{\tau_{1}}\right)_{\#}\right)(1,0)=\psi(2,0)=\psi(1,0)^{2}=(a \sigma)^{2}=a \sigma a \sigma^{-1} \sigma^{2}=a l_{\sigma}(a) \sigma^{2}=\varphi(1,0), \\
& \left(\psi \circ\left(p_{\tau_{1}}\right)_{\#}\right)(0,1)=\psi(0,1)=\varphi(0,1)
\end{aligned}
$$

e

$$
\begin{aligned}
& (\pi \circ \psi)(1,0)=\pi(a \sigma)=\overline{1}=\theta_{\tau_{1}}(1,0), \\
& (\pi \circ \psi)(0,1)=\pi(b)=\overline{0}=\theta_{\tau_{1}}(0,1) .
\end{aligned}
$$

Proposição 2.2.2. Se $\alpha \in\left[\mathbb{T}^{2}, * ; \mathbb{T}^{2}, *\right]$, então $\alpha$ não tem a propriedade de Borsuk-Ulam com respeito a involução $\tau_{1}$.

Demonstração. Seja $h_{\alpha}=\Gamma(\alpha): \pi_{1}\left(\mathbb{T}^{2}\right) \rightarrow \pi_{1}\left(\mathbb{T}^{2}\right)$. Sejam também $m_{1}, n_{1}, m_{2}, n_{2} \in \mathbb{Z}$ tais que:

$$
h_{\alpha}(1,0)=\left(m_{1}, n_{1}\right) \quad \text { e } \quad h_{\alpha}(0,1)=\left(m_{2}, n_{2}\right) .
$$

Sejam ainda $r, s \in \mathbb{Z}$ e $i, j \in\{0,1\}$ tais que

$$
m_{1}=2 r+i \quad \text { e } \quad n_{1}=2 s+j .
$$

Em $P_{2}\left(\mathbb{T}^{2}\right)$ tomamos os elementos

$$
a=\left(x^{i} y^{j}, r, s\right) \quad \text { e } \quad b=\left(\mathbb{1}, m_{2}, n_{2}\right) .
$$


Vamos usar a Observação 2.1.2 para mostrar que $a, b$ e $h_{\alpha}$ satisfazem as condições $(i)$, (ii) e (iii) da Proposição 2.2.1, e portanto, pelo mesmo resultado, seguirá que $\alpha$ não tem a propriedade de Borsuk-Ulam. Temos que

$$
a l_{\sigma}(b)=\left(x^{i} y^{j}, r, s\right) l_{\sigma}\left(\mathbb{1}, m_{2}, n_{2}\right)=\left(x^{i} y^{j}, r, s\right)\left(\mathbb{1}, m_{2}, n_{2}\right)=\left(\mathbb{1}, m_{2}, n_{2}\right)\left(x^{i} y^{j}, r, s\right)=b a
$$

e portanto, vale $(i)$. Notemos que

$$
\begin{aligned}
a l_{\sigma}(a) & =\left(x^{i} y^{j}, r, s\right) l_{\sigma}\left(x^{i} y^{j}, r, s\right) \\
& =\left(x^{i} y^{j}, r, s\right) l_{\sigma}\left(x^{i}, 0,0\right) l_{\sigma}\left(y^{j}, 0,0\right) l_{\sigma}(\mathbb{1}, r, s) \\
& =\left(x^{i} y^{j}, r, s\right)\left(\left(B x^{-1}\right)^{i}, i, 0\right)\left(\left(B y^{-1}\right)^{j}, 0, j\right)(\mathbb{1}, r, s) \\
& =\left(x^{i} y^{j}\left(B x^{-1}\right)^{i}\left(B y^{-1}\right)^{j}, 2 r+i, 2 s+j\right) \\
& =\left(x^{i} y^{j}\left(B x^{-1}\right)^{i}\left(B y^{-1}\right)^{j}, m_{1}, n_{1}\right) .
\end{aligned}
$$

Assim, temos que

$$
\left(p_{1}\right)_{\#}\left(a l_{\sigma}(a)\right)=\left(p_{1}\right)_{\#}\left(x^{i} y^{j}\left(B x^{-1}\right)^{i}\left(B y^{-1}\right)^{j}, m_{1}, n_{1}\right)=\left(m_{1}, n_{1}\right)=h_{\alpha}(1,0)
$$

e portanto, vale (ii). Por fim, temos que

$$
\left(p_{1}\right)_{\#}(b)=\left(p_{1}\right)_{\#}\left(\mathbb{1}, m_{2}, n_{2}\right)=\left(m_{2}, n_{2}\right)=h_{\alpha}(0,1)
$$

e logo, vale (iii), o que encerra a demonstração.

Decorre imediatamente do Teorema 1.4.4 item 2., da Observação 2.1.1 e da Proposição 2.2.2 o seguinte resultado de classificação:

Teorema 2.2.3. Seja $\tau_{1}: \mathbb{T}^{2} \rightarrow \mathbb{T}^{2}$ a involução livre de pontos fixos definida por $\tau_{1}(x, y)=\left(x+\frac{1}{2}, y\right)$. Então toda classe de homotopia $\beta \in\left[\mathbb{T}^{2}, \mathbb{T}^{2}\right]$ não tem a propriedade de Borsuk-Ulam com respeito a $\tau_{1}$.

\subsection{Classificação das classes de homotopia com a propriedade de Borsuk-Ulam com respeito a $\tau_{2}$}

Nesta seção, nós vamos classificar os elementos do conjunto $\left[\mathbb{T}^{2}, \mathbb{T}^{2}\right]$ em que vale a propriedade de Borsuk-Ulam com respeito a involução $\tau_{2}$. Para tal objetivo, o roteiro será o mesmo da Seção 2.2. Em todas as demonstrações usaremos fortemente a Observação 2.1.2.

Proposição 2.3.1. Seja $\alpha \in\left[\mathbb{T}^{2}, * ; \mathbb{T}^{2}, *\right]$ uma classe de homotopia pontuada e seguindo a notação da Observação 2.1.1, seja $h_{\alpha}=\Gamma(\alpha): \pi_{1}\left(\mathbb{T}^{2}\right) \rightarrow \pi_{1}\left(\mathbb{T}^{2}\right)$. Então $\alpha$ não tem a propriedade de Borsuk-Ulam com respeito a $\tau_{2}$ se, e somente se, existem tranças puras $a, b \in P_{2}\left(\mathbb{T}^{2}\right)$ tais que:

(i) $a b l_{\sigma}(a)=b$;

(ii) $h_{\alpha}(1,0)=\left(p_{1}\right)_{\#}(a)$;

(iii) $h_{\alpha}(0,1)=\left(p_{1}\right)_{\#}\left(b l_{\sigma}(b)\right)$.

Demonstração. Seja $\alpha$ uma classe de homotopia pontuada que não tem a propriedade de BorsukUlam e vamos mostrar que existem tranças $a, b \in P_{2}\left(\mathbb{T}^{2}\right)$ tais que são verdadeiras as condições $(i)$, 
(ii) e (iii). Pelo Lema Fundamental 1.4.3, existe o seguinte diagrama comutativo:

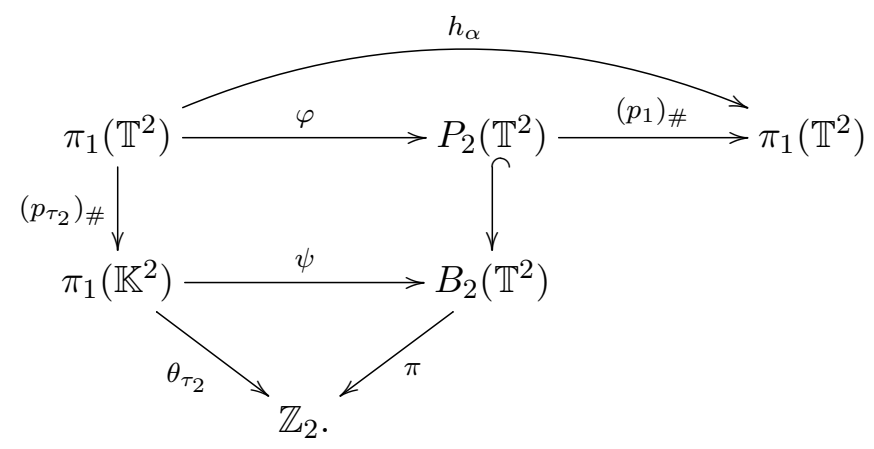

Por $(2.2)$, como $\theta_{\tau_{2}}(1,0)=\overline{0}$ e $\theta_{\tau_{2}}(0,1)=\overline{1}$, então pela comutatividade do diagrama $(2.4), \psi(1,0)$ deve ser uma trança pura e $\psi(0,1)$ deve ser uma trança não pura. Logo, pela Observação 2.1.3, existem tranças puras $a, b \in P_{2}\left(\mathbb{T}^{2}\right)$ tais que

(1) $\psi(1,0)=a$;

(2) $\psi(0,1)=b \sigma$.

Como em $\mathbb{Z} \rtimes \mathbb{Z}$ vale a relação $[(1,0),(0,1)]^{\prime}=(0,0)$, então

$$
1=[\psi(1,0), \psi(0,1)]^{\prime}=[a, b \sigma]^{\prime}=a b \sigma a \sigma^{-1} b^{-1}=a b l_{\sigma}(a) b^{-1} .
$$

Portanto, vale

(i) $a b l_{\sigma}(a)=b$.

Novamente por $(2.2)$, como $\left(p_{\tau_{2}}\right)_{\#}(1,0)=(1,0)$ e $\left(p_{\tau_{2}}\right)_{\#}(0,1)=(0,2)$, segue da comutatividade de (2.4) que valem as seguintes igualdades para o homomorfismo $\varphi$ :

(3) $\varphi(1,0)=\left(\psi \circ\left(p_{\tau_{2}}\right)_{\#}\right)(1,0)=\psi(1,0)=a$;

(4) $\varphi(0,1)=\left(\psi \circ\left(p_{\tau_{2}}\right)_{\#}\right)(0,1)=\psi(0,2)=\psi(0,1)^{2}=(b \sigma)^{2}=b \sigma b \sigma^{-1} \sigma^{2}=b l_{\sigma}(b) \sigma^{2}$.

Novamente por (2.4) e pela Observação 2.1.2, valem as seguintes igualdades para o homomorfismo $h_{\alpha}$ :

(ii) $h_{\alpha}(1,0)=\left(\left(p_{1}\right)_{\#} \circ \varphi\right)(1,0)=\left(p_{1}\right)_{\#}(a)$;

(iii) $h_{\alpha}(0,1)=\left(\left(p_{1}\right)_{\#} \circ \varphi\right)(1,0)=\left(p_{1}\right)_{\#}\left(b l_{\sigma}(b) \sigma^{2}\right)=\left(p_{1}\right)_{\#}\left(b l_{\sigma}(b)\right)+\left(p_{1}\right)_{\#}(B, 0,0)=\left(p_{1}\right)_{\#}\left(b l_{\sigma}(b)\right)$.

Reciprocamente, suponhamos que existam tranças $a, b \in P_{2}\left(\mathbb{T}^{2}\right)$ e valem $(i)$, (ii) e (iii). Utilizando a Observação 2.1.3, definimos

e

$$
\begin{aligned}
\varphi: \pi_{1}\left(\mathbb{T}^{2}\right) & \longrightarrow P_{2}\left(\mathbb{T}^{2}\right) \\
(1,0) & \longmapsto a \\
(0,1) & \longmapsto b l_{\sigma}(b) \sigma^{2}
\end{aligned}
$$

$$
\begin{aligned}
\psi: \pi_{1}\left(\mathbb{K}^{2}\right) & \longrightarrow B_{2}\left(\mathbb{T}^{2}\right) \\
(1,0) & \longmapsto a \\
(0,1) & \longmapsto b \sigma .
\end{aligned}
$$

Mostremos que de fato $\varphi$ e $\psi$ definem homomorfismos. Pela Observação 2.1.2, temos que:

$$
\begin{aligned}
{[\varphi(1,0), \varphi(0,1)] } & =\left[a, b l_{\sigma}(b) \sigma^{2}\right]=a b \sigma b \sigma^{-1} \sigma^{2} a^{-1} \sigma^{-2} \sigma b^{-1} \sigma^{-1} b^{-1} \\
& =a b l_{\sigma}\left(b l_{\sigma}(a)^{-1} b^{-1}\right) b^{-1} \stackrel{(i)}{=} a b l_{\sigma}(a) b^{-1} \stackrel{(i)}{=} b b^{-1}=1
\end{aligned}
$$


e

$$
[\psi(1,0), \psi(0,1)]^{\prime}=[a, b \sigma]^{\prime}=a b \sigma a \sigma^{-1} b^{-1}=a b l_{\sigma}(a) b^{-1} \stackrel{(i)}{=} b b^{-1}=1 .
$$

Vamos mostrar que temos um diagrama comutativo como em (2.4), donde seguirá pelo Lema Fundamental 1.4.3 que $\alpha$ não tem a propriedade de Borsuk-Ulam. Utilizaremos as Observações $2.1 .2 \mathrm{e}$ 2.1.3 e também os valores dos homomorfismos $\left(p_{\tau_{2}}\right)_{\#}$ e $\theta_{\tau_{2}}$, os quais estão definidos em (2.2). Temos

$$
\begin{aligned}
& \left(\left(p_{1}\right)_{\#} \circ \varphi\right)(1,0)=\left(p_{1}\right)_{\#}(a) \stackrel{(i i)}{=} h_{\alpha}(1,0), \\
& \left(\left(p_{1}\right)_{\#} \circ \varphi\right)(0,1)=\left(p_{1}\right)_{\#}\left(b l_{\sigma}(b) \sigma^{2}\right)=\left(p_{1}\right)_{\#}\left(b l_{\sigma}(b)\right)+\left(p_{1}\right)_{\#}(B, 0,0) \stackrel{(i i i)}{=} h_{\alpha}(0,1), \\
& \left(\psi \circ\left(p_{\tau_{2}}\right)_{\#}\right)(1,0)=\psi(1,0)=a=\varphi(1,0), \\
& \left(\psi \circ\left(p_{\tau_{2}}\right)_{\#}\right)(0,1)=\psi(2,0)=\psi(1,0)^{2}=(b \sigma)^{2}=b \sigma b \sigma^{-1} \sigma^{2}=b l_{\sigma}(b) \sigma^{2}=\varphi(0,1)
\end{aligned}
$$

$\mathrm{e}$

$$
\begin{aligned}
& (\pi \circ \psi)(1,0)=\pi(a)=\overline{0}=\theta_{\tau_{1}}(1,0), \\
& (\pi \circ \psi)(0,1)=\pi(b \sigma)=\overline{1}=\theta_{\tau_{1}}(0,1) .
\end{aligned}
$$

Afim de facilitar alguns cálculos, vamos fazer algumas considerações sobre o operador $l_{\sigma}: P_{2}\left(\mathbb{T}^{2}\right) \rightarrow P_{2}\left(\mathbb{T}^{2}\right)$. Seja $w=w(x, y) \in F(x, y)$. Denotemos por $|w|_{x}$ a soma dos expoentes dos geradores $x$ que aparecem na palavra $w$ e por $|w|_{y}$ a soma dos expoentes dos geradores $y$ que aparecem na palavra $w$. Pela Observação 2.1.2, temos que

$$
l_{\sigma}(x, 0,0)=\left(B x^{-1}, 1,0\right)=\left(x y^{-1} x^{-1} y x^{-1}, 1,0\right)=\left(\left(x y^{-1}\right) x^{-1}\left(y x^{-1}\right), 1,0\right)
$$

e

$$
l_{\sigma}(y, 0,0)=\left(B y^{-1}, 0,1\right)=\left(x y^{-1} x^{-1} y y^{-1}, 0,1\right)=\left(\left(x y^{-1}\right) y^{-1}\left(y x^{-1}\right), 0,1\right) .
$$

e portanto, temos que

$$
l_{\sigma}(w, 0,0)=l_{\sigma}(w(x, y), 0,0)=\left(x y^{-1} w\left(x^{-1}, y^{-1}\right) y x^{-1},|w|_{x},|w|_{y}\right) .
$$

Para cada classe de homotopia pontuada $\alpha \in\left[\mathbb{T}^{2}, * ; \mathbb{T}^{2}, *\right]$, consideremos o homomorfismo $h_{\alpha}=\Gamma(\alpha): \pi_{1}\left(\mathbb{T}^{2}\right) \rightarrow \pi_{1}\left(\mathbb{T}^{2}\right)$ e sejam $m_{1}, n_{1}, m_{2}, n_{2} \in \mathbb{Z}$ tais que

$$
h_{\alpha}(1,0)=\left(m_{1}, n_{1}\right) \quad \text { e } \quad h_{\alpha}(0,1)=\left(m_{2}, n_{2}\right) .
$$

Nós faremos a classificação das classes de homotopia $\alpha$ que tem (ou não) a propriedade de Borsuk-Ulam separando em casos conforme a paridade de $m_{2}$ e $n_{2}$. No caso em que $m_{2}$ e $n_{2}$ são ambos pares faremos a análise em duas partes, conforme a nulidade de $m_{1}$ e $n_{1}$. As hipóteses das Proposições $2.3 .2,2.3 .3,2.3 .4,2.3 .5$ e 2.3 .9 esgotarão todas as possibilidades para os valores $m_{1}$, $n_{1}, m_{2}$ e $n_{2}$.

Proposição 2.3.2. Se $m_{2}$ e $n_{2}$ são ímpares, então $\alpha$ não tem a propriedade de Borsuk-Ulam.

Demonstração. Por hipótese, existem $r, s \in \mathbb{Z}$ tais que $m_{2}=2 r+1$ e $n_{2}=2 s+1$. Consideremos os seguintes elementos de $P_{2}\left(\mathbb{T}^{2}\right)$ :

$$
a=\left(x^{-2 m_{1}} y^{-2 n_{1}}, m_{1}, n_{1}\right) \quad \text { e } \quad b=\left(y^{1+2 n_{1}} x^{-1}, r+1, s-n_{1}\right) .
$$


Pela Observação 2.1.2 e pela igualdade (2.5) temos que

$$
\begin{aligned}
a b l_{\sigma}(a) & =\left(x^{-2 m_{1}} y^{-2 n_{1}}, m_{1}, n_{1}\right)\left(y^{1+2 n_{1}} x^{-1}, r+1, s-n_{1}\right) l_{\sigma}\left(x^{-2 m_{1}} y^{-2 n_{1}}, m_{1}, n_{1}\right) \\
& =\left(x^{-2 m_{1}} y x^{-1}, m_{1}+r+1, s\right)\left(x y^{-1} x^{2 m_{1}} y^{2 n_{1}} y x^{-1},-2 m_{1}+m_{1},-2 n_{1}+n_{1}\right) \\
& =\left(y^{1+2 n_{1}} x^{-1}, r+1, s-n_{1}\right)=b .
\end{aligned}
$$

Portanto, vale a condição $(i)$ da Proposição 2.3.1. Temos também que

$$
\left(p_{1}\right)_{\#}(a)=\left(p_{1}\right)_{\#}\left(x^{-2 m_{1}} y^{-2 n_{1}}, m_{1}, n_{1}\right)=\left(m_{1}, n_{1}\right)=h_{\alpha}(1,0)
$$

e logo, vale (ii). Por fim, usando a igualdalde (2.5) temos que

$$
\begin{aligned}
\left(p_{1}\right)_{\#}\left(b l_{\sigma}(b)\right) & =\left(p_{1}\right)_{\#}\left(\left(y^{1+2 n_{1}} x^{-1}, r+1, s-n_{1}\right) l_{\sigma}\left(y^{1+2 n_{1}} x^{-1}, r+1, s-n_{1}\right)\right) \\
& =\left(r+1, s-n_{1}\right)+\left(-1,1+2 n_{1}\right)+\left(r+1, s-n_{1}\right) \\
& =(2 r+1,2 s+1)=\left(m_{2}, n_{2}\right)=h_{\alpha}(0,1)
\end{aligned}
$$

e portanto, vale (iii). Pela Proposição 2.3.1, segue que $\alpha$ não tem a propriedade de Borsuk-Ulam.

Proposição 2.3.3. Se $m_{2}$ é ímpar e $n_{2}$ é par, então a não tem a propriedade de Borsuk-Ulam.

Demonstração. Por hipótese, existem $r, s \in \mathbb{Z}$ tais que $m_{2}=2 r+1$ e $n_{2}=2 s$. Consideremos os seguintes elementos de $P_{2}\left(\mathbb{T}^{2}\right)$ :

$$
a=\left(x^{-m_{1}} y^{-2 n_{1}-1} x^{-m_{1}} y, m_{1}, n_{1}\right) \quad \text { e } \quad b=\left(x^{-1}, r+1, s\right) .
$$

Pela Observação 2.1.2 e pela igualdade (2.5) temos que

$$
\begin{aligned}
a b l_{\sigma}(a) & =\left(x^{-m_{1}} y^{-2 n_{1}-1} x^{-m_{1}} y, m_{1}, n_{1}\right)\left(x^{-1}, r+1, s\right) l_{\sigma}\left(x^{-m_{1}} y^{-2 n_{1}-1} x^{-m_{1}} y, m_{1}, n_{1}\right) \\
& =\left(x^{-m_{1}} y^{-2 n_{1}-1} x^{-m_{1}} y x^{-1}, m_{1}+r+1, n_{1}+s\right)\left(x y^{-1} x^{m_{1}} y^{2 n_{1}+1} x^{m_{1}} y^{-1} y x^{-1},-m_{1},-n_{1}\right) \\
& =\left(x^{-1}, r+1, s\right)=b .
\end{aligned}
$$

Portanto, vale a condição $(i)$ da Proposição 2.3.1. Temos também que

$$
\left(p_{1}\right)_{\#}(a)=\left(p_{1}\right)_{\#}\left(x^{-m_{1}} y^{-2 n_{1}-1} x^{-m_{1}} y, m_{1}, n_{1}\right)=\left(m_{1}, n_{1}\right)=h_{\alpha}(1,0)
$$

e logo, vale (ii). Por fim, usando a igualdalde (2.5) temos que

$$
\begin{aligned}
\left(p_{1}\right)_{\#}\left(b l_{\sigma}(b)\right) & =\left(p_{1}\right)_{\#}\left(\left(x^{-1}, r+1, s\right) l_{\sigma}\left(x^{-1}, r+1, s\right)\right) \\
& =(r+1, s)+(-1,0)+(r+1, s) \\
& =(2 r+1,2 s)=\left(m_{2}, n_{2}\right)=h_{\alpha}(0,1)
\end{aligned}
$$

e portanto, vale (iii). Pela Proposição 2.3.1, segue que $\alpha$ não tem a propriedade de Borsuk-Ulam.

Proposição 2.3.4. Se $m_{2}$ é par e $n_{2}$ é ímpar, então a não tem a propriedade de Borsuk-Ulam.

Demonstração. Por hipótese, existem $r, s \in \mathbb{Z}$ tais que $m_{2}=2 r$ e $n_{2}=2 s+1$. Consideremos os seguintes elementos de $P_{2}\left(\mathbb{T}^{2}\right)$ :

$$
a=\left(x^{-2 m_{1}+1} y^{-n_{1}} x^{-1} y^{-n_{1}}, m_{1}, n_{1}\right) \quad \text { e } \quad b=\left(x^{-2 m_{1}+1} y x^{-1}, m_{1}+r, s\right) .
$$

Pela Observação 2.1.2 e pela igualdade (2.5) temos que

$$
\begin{aligned}
a b l_{\sigma}(a) & =\left(x^{-2 m_{1}+1} y^{-n_{1}} x^{-1} y^{-n_{1}}, m_{1}, n_{1}\right)\left(x^{-2 m_{1}+1} y x^{-1}, m_{1}+r, s\right) l_{\sigma}\left(x^{-2 m_{1}+1} y^{-n_{1}} x^{-1} y^{-n_{1}}, m_{1}, n_{1}\right) \\
& =\left(x^{-2 m_{1}+1} y^{-n_{1}} x^{-1} y^{-n_{1}} x^{-2 m_{1}+1} y x^{-1}, 2 m_{1}+r, n_{1}+s\right)\left(x y^{-1} x^{2 m_{1}-1} y^{n_{1}} x y^{n_{1}} y x^{-1},-m_{1},-n_{1}\right) \\
& =\left(x^{-2 m_{1}+1} y x^{-1}, m_{1}+r, s\right)=b .
\end{aligned}
$$


Portanto, vale a condição $(i)$ da Proposição 2.3.1. Temos também que

$$
\left(p_{1}\right)_{\#}(a)=\left(p_{1}\right)_{\#}\left(x^{-2 m_{1}+1} y^{-n_{1}} x^{-1} y^{-n_{1}}, m_{1}, n_{1}\right)=\left(m_{1}, n_{1}\right)=h_{\alpha}(1,0)
$$

e logo, vale (ii). Por fim, usando a igualdalde (2.5) temos que

$$
\begin{aligned}
\left(p_{1}\right)_{\#}\left(b l_{\sigma}(b)\right) & =\left(p_{1}\right)_{\#}\left(\left(x^{-2 m_{1}+1} y x^{-1}, m_{1}+r, s\right) l_{\sigma}\left(x^{-2 m_{1}+1} y x^{-1}, m_{1}+r, s\right)\right) \\
& =\left(m_{1}+r, s\right)+\left(-2 m_{1}, 1\right)+\left(m_{1}+r, s\right) \\
& =(2 r, 2 s+1)=\left(m_{2}, n_{2}\right)=h_{\alpha}(0,1)
\end{aligned}
$$

e portanto, vale (iii). Pela Proposição 2.3.1, segue que $\alpha$ não tem a propriedade de Borsuk-Ulam.

Proposição 2.3.5. Se $m_{2}$ e $n_{2}$ são pares e $m_{1}=n_{1}=0$, então $\alpha$ não tem a propriedade de Borsuk-Ulam.

Demonstração. Por hipótese, existem $r, s \in \mathbb{Z}$ tais que $m_{2}=2 r$ e $n_{2}=2 s$. Consideremos os seguintes elementos de $P_{2}\left(\mathbb{T}^{2}\right)$ :

$$
a=(\mathbb{1}, 0,0) \quad \text { e } \quad b=(\mathbb{1}, r, s) .
$$

Pela Observação 2.1.2 temos que

$$
a b l_{\sigma}(a)=(\mathbb{1}, 0,0)(\mathbb{1}, r, s) l_{\sigma}(\mathbb{1}, 0,0)=(\mathbb{1}, r, s)=b .
$$

Portanto, vale a condição $(i)$ da Proposição 2.3.1. Temos também que

$$
\left(p_{1}\right)_{\#}(a)=\left(p_{1}\right)_{\#}(\mathbb{1}, 0,0)=(0,0)=\left(m_{1}, n_{1}\right)=h_{\alpha}(1,0)
$$

e logo, vale $(i i)$. Por fim, temos que

$$
\left(p_{1}\right)_{\#}\left(b l_{\sigma}(b)\right)=\left(p_{1}\right)_{\#}\left((\mathbb{1}, r, s) l_{\sigma}(\mathbb{1}, r, s)\right)=(r, s)+(r, s)=(2 r, 2 s)=\left(m_{2}, n_{2}\right)=h_{\alpha}(0,1)
$$

e portanto, vale (iii). Pela Proposição 2.3.1, segue que $\alpha$ não tem a propriedade de Borsuk-Ulam.

Antes de provarmos a Proposição 2.3.9, vamos fazer uma definição e provar dois resultados que serão úteis.

Definição 2.3.6. Seja $z=z(x, y) \in F(x, y)$. Dizemos que z é um palíndromo se a palavra $z$ pode ser lida da esquerda para direita e da direita para a esquerda, ou equivalentemente, $z(x, y)=z\left(x^{-1}, y^{-1}\right)^{-1}$.

Lema 2.3.7. Se $z=z(x, y) \in F(x, y)$ é um palíndromo, então $|z|_{x}$ ou $|z|_{y}$ é par.

Demonstração. Se $z$ é trivial ou $z=k$, com $k \in\left\{x, x^{-1}, y, y^{-1}\right\}$, então é claro que $|z|_{x}$ ou $|z|_{y}$ é par. Lembremos que as palavras de comprimento 2 de $F(x, y)$ são $x^{2 a}, y^{2 b}, x^{a} y^{b}$ e $y^{b} x^{a}$, sendo $a, b \in\{-1,1\}$. Logo, se $z$ é um palíndromo de comprimento 2 , então $z=x^{2 a}$ ou $y^{2 b}$, e portanto, $|z|_{x}$ ou $|z|_{y}$ é par. Suponhamos então o resultado verdadeiro para palíndromos de comprimento entre 0 e $r$, com $r \geq 2$, e vamos mostrar por indução que o resultado é verdadeiro para palíndromos de comprimento $r+1$. Seja então $z$ uma palavra de comprimento $r+1$, escrita de forma reduzida, tal que $z=z\left(x^{-1}, y^{-1}\right)^{-1}$. Então, para algum $k \in\left\{x, x^{-1}, y, y^{-1}\right\}$ e para algum $z_{1} \in F(x, y)$ temos que

$$
z=k z_{1} k
$$

O comprimento de $z_{1}$ é $r-2$ e notemos que

$$
k z_{1} k=z=z\left(x^{-1}, y^{-1}\right)^{-1}=\left(k^{-1} z_{1}\left(x^{-1}, y^{-1}\right) k^{-1}\right)^{-1}=k z_{1}\left(x^{-1}, y^{-1}\right)^{-1} k .
$$


Logo, $z_{1}=z_{1}\left(x^{-1}, y^{-1}\right)^{-1}$, ou seja, $z_{1}$ é um palíndromo e pela hipótese de indução temos que $\left|z_{1}\right|_{x}$ ou $\left|z_{1}\right|_{y}$ é par. Notemos que

$$
|z|_{x}=\left|k z_{1} k\right|_{x}=2|k|_{x}+\left|z_{1}\right|_{x} \equiv\left|z_{1}\right|_{x} \bmod 2
$$

e

$$
|z|_{y}=\left|k z_{1} k\right|_{y}=2|k|_{y}+\left|z_{1}\right|_{y} \equiv\left|z_{1}\right|_{y} \bmod 2
$$

e portanto, $|z|_{x}$ ou $|z|_{y}$ é par.

Lema 2.3.8. Sejam $z, w \in F(x, y)$ tais que w é não trivial, $|w|_{x}$ e $|w|_{y}$ são pares e

$$
z w z^{-1}=w\left(x^{-1}, y^{-1}\right)^{-1} .
$$

Então $|z|_{x}$ ou $|z|_{y}$ é par.

Demonstração. Se $z$ é trivial, o resultado é imediato. Suponhamos então que $z$ não é trivial. Sem perda de generalidade, podemos supor que $z$ e $w$ estão escritas na forma reduzida. Como o comprimento de $w$ é igual ao comprimento de $w\left(x^{-1}, y^{-1}\right)^{-1}$, então ocorre cancelamento no lado esquerdo da equação (2.6). Como estamos interessados na paridade de $|z|_{x}$ e de $|z|_{y}$, vamos fazer algumas manipulações na equação (2.6) que não alteram o resultado.

PASSO 1: Podemos supor que ocorre cancelamento na sub-palavra zw. Suponhamos que ocorra cancelamento na sub-palavra $w z^{-1}$. Seja $w^{\prime}=w^{-1}$. É claro que $w^{\prime}$ é uma palavra não trivial e $\left|w^{\prime}\right|_{x}$ e $\left|w^{\prime}\right|_{y}$ são pares. Notemos que

$$
z w^{\prime} z^{-1}=z w^{-1} z^{-1}=\left(z w z^{-1}\right)^{-1}=w\left(x^{-1}, y^{-1}\right)=w^{\prime}\left(x^{-1}, y^{-1}\right)^{-1},
$$

ou seja, temos uma equação no mesmo formato que em (2.6) e ocorre cancelamento em $z w^{\prime}$.

PASSO 2: Podemos supor que ou ocorre cancelamento na sub-palavra zw ou ocorre cancelamento na sub-palavra $w z^{-1}$. Suponhamos que ocorram cancelamentos nas sub-palavras $z w$ e $w z^{-1}$. Então $z=z_{1} k$ e $w=k^{-1} w_{1} k$ para algum $k \in\left\{x, x^{-1}, y, y^{-1}\right\}$ e para algum $z_{1}, w_{1} \in F(x, y)$. Notemos que $w_{1}$ é uma palavra não trivial e $\left|w_{1}\right|_{x}$ e $\left|w_{1}\right|_{y}$ são pares. Por (2.6) temos que

$$
\begin{aligned}
z_{1} k k^{-1} w_{1} k k^{-1} z_{1}^{-1} & =k w_{1}\left(x^{-1}, y^{-1}\right)^{-1} k^{-1} \\
& \Uparrow \\
\left(k^{-1} z_{1}\right) w_{1}\left(k^{-1} z_{1}\right)^{-1} & =w_{1}\left(x^{-1}, y^{-1}\right)^{-1},
\end{aligned}
$$

ou seja, temos uma equação no mesmo formato que em (2.6). Para cada $l \in\{x, y\}$, temos que

$$
\left|k^{-1} z_{1}\right|_{l}=-|k|_{l}+\left|z_{1}\right|_{l}=-2|k|_{l}+\left|z_{1}\right|_{l}+|k|_{l}=-2|k|_{l}+\left|z_{1} k\right|_{l}=-2|k|_{l}+|z|_{l} \equiv|z|_{l} \bmod 2 .
$$

Se houver cancelamentos nas sub-palavras $\left(k^{-1} z_{1}\right) w_{1}$ e $w_{1}\left(k^{-1} z_{1}\right)^{-1}$, repetimos o argumento. Depois de uma quantidade finita de passos (que pode ser nula e é no máximo igual ao comprimento de $z$ ), podemos garantir que existem $z^{\prime}, w^{\prime} \in F(x, y)$, escritas de forma reduzida, tais que $w^{\prime}$ é não trivial, $\left|w^{\prime}\right|_{x}$ e $\left|w^{\prime}\right|_{y}$ são pares,

$$
z^{\prime} w^{\prime} z^{\prime-1}=w^{\prime}\left(x^{-1}, y^{-1}\right)^{-1}
$$

$\left|z^{\prime}\right|_{l} \equiv|z|_{l} \bmod 2$, sendo $l \in\{x, y\}$, e se houver cancelamentos ou é na sub-palavra $z^{\prime} w^{\prime}$ ou é na sub-palavra $w^{\prime} z^{\prime-1}$.

PASSO 3: Podemos supor que $z$ não se escreve de forma reduzida como $z^{\prime} w^{\varepsilon}$, sendo $\varepsilon \in\{-1,1\}$. Se $z$ se escreve de forma reduzida como $z_{1} w^{\varepsilon_{1}}, \varepsilon_{1} \in\{-1,1\}$, então por (2.6), temos que

$$
z_{1} w z_{1}^{-1}=z_{1} w^{\varepsilon} w\left(z_{1} w^{\varepsilon_{1}}\right)^{-1}=z w z^{-1}=w\left(x^{-1}, y^{-1}\right)^{-1}
$$


ou seja, temos uma equação no formato de (2.6). Para cada $l \in\{x, y\}$, como $|w|_{l}$ é par, então

$$
|z|_{l}=\left|z_{1} w^{\varepsilon_{1}}\right|_{l}=\left|z_{1}\right|_{l}+\varepsilon_{1}|w|_{l} \equiv\left|z_{1}\right|_{l} \bmod 2 .
$$

Se $z_{1}$ se escreve de forma reduzida como $z_{2} w^{\varepsilon_{2}}, \varepsilon_{2} \in\{-1,1\}$, repetimos o argumento. Depois de uma quantidade finita de passos, podemos garantir que existem $\tilde{z} \in F(x, y)$, escrito de forma reduzida, tal que

$$
\tilde{z} w \tilde{z}^{-1}=w\left(x^{-1}, y^{-1}\right)^{-1}
$$

$|z|_{l} \equiv|\tilde{z}|_{l} \bmod 2$ e não é possível escrever de forma reduzida $\tilde{z}=z_{k} w^{\varepsilon_{k}}, \varepsilon_{k} \in\{-1,1\}$.

Após aplicar os passos 1, 2 e 3 quantas vezes forem necessárias, nós podemos supor, sem perda de generalidade, que $z$ e $w$ se escrevem de forma reduzida como

$$
z=z_{1} z_{2} \quad \text { e } \quad w=w_{1} w_{2}
$$

sendo que $w_{1}$ e $w_{2}$ são ambos não triviais, $w_{1}=z_{2}^{-1}$ e não ocorre cancelamentos nas palavras $z_{1} w_{2}$ e $w_{2} z_{2}^{-1}$. Assim, por (2.6), temos que

$$
\begin{aligned}
z w z^{-1} & =w\left(x^{-1}, y^{-1}\right)^{-1} \\
z_{1} z_{2} w_{1} w_{2} z_{2}^{-1} z_{1}^{-1} & =\left(w_{1}\left(x^{-1}, y^{-1}\right) w_{2}\left(x^{-1}, y^{-1}\right)\right)^{-1} \\
z_{1} z_{2} z_{2}^{-1} w_{2} w_{1} z_{1}^{-1} & =w_{2}\left(x^{-1}, y^{-1}\right)^{-1} w_{1}\left(x^{-1}, y^{-1}\right)^{-1} \\
z_{1} w_{2} w_{1} z_{1}^{-1} & =w_{2}\left(x^{-1}, y^{-1}\right)^{-1} w_{1}\left(x^{-1}, y^{-1}\right)^{-1}
\end{aligned}
$$

Por hipótese não há cancelamento em $z_{1} w_{2}$. Também não ocorre cancelamento em $w_{1} z_{1}^{-1}$, pois $w_{1}=z_{2}^{-1}$ e tomamos $z_{1} z_{2}$ escrito de forma reduzida. Não ocorre cancelamento em $w_{2} w_{1}$, pois $w_{1}=z_{2}^{-1}$ e não ocorre cancelamento em $w_{2} z_{2}^{-1}$. Portanto, não acontecem cancelamentos na palavra $z_{1} w_{2} w_{1} z_{1}^{-1}$. Mas como o comprimento de $w_{2} w_{1}$ é igual ao comprimento de $w_{1} w_{2}$, que por sua vez é igual ao comprimento de $w_{2}\left(x^{-1}, y^{-1}\right)^{-1} w_{1}\left(x^{-1}, y^{-1}\right)^{-1}$, por (2.7) segue que

$$
z_{1} \text { é trivial e } \quad w_{2} w_{1}=w_{2}\left(x^{-1}, y^{-1}\right)^{-1} w_{1}\left(x^{-1}, y^{-1}\right)^{-1} .
$$

Como o comprimento de $w_{i}$ é igual ao comprimento de $w_{i}\left(x^{-1}, y^{-1}\right)^{-1}, i \in\{1,2\}$, e não há cancelamento em $w_{2} w_{1}$ e nem em $w_{2}\left(x^{-1}, y^{-1}\right)^{-1} w_{1}\left(x^{-1}, y^{-1}\right)^{-1}$, segue que

$$
w_{i}=w_{i}\left(x^{-1}, y^{-1}\right)^{-1}, \quad i \in\{1,2\},
$$

ou seja, $w_{1}$ e $w_{2}$ são palíndromos. Por fim, como $z=z_{1} z_{2}=w_{1}^{-1}$, então $z$ é um palíndromo e pelo Lema 2.3.7, segue que $|z|_{x}$ ou $|z|_{y}$ é par.

Proposição 2.3.9. Se $m_{2}$ e $n_{2}$ são pares e $\left(m_{1}, n_{1}\right) \neq(0,0)$, então $\alpha$ tem a propriedade de BorsukUlam.

Demonstração. Faremos a prova por contradição. Suponhamos então que $\alpha$ não tem a propriedade de Borsuk-Ulam. Pela Proposição 2.3.1, existem tranças puras

$$
a=\left(w(x, y), r_{a}, s_{a}\right) \quad \text { e } \quad b=\left(z(x, y), r_{b}, s_{b}\right)
$$

tais que $a, b$ e $h_{\alpha}$ satisfazem $(i),(i i)$ e $(i i i)$. Lembremos que

$$
h_{\alpha}:\left\{\begin{array}{l}
(1,0) \mapsto\left(m_{1}, n_{1}\right) \\
(0,1) \mapsto\left(m_{2}, n_{2}\right) .
\end{array}\right.
$$

Da condição (ii) e pela Observação 2.1.2 segue que

$$
a=\left(w, m_{1}, n_{1}\right) .
$$


Pela condição (iii) e pela igualdade (2.5), temos que

$$
\begin{aligned}
\left(m_{2}, n_{2}\right) & =\left(p_{1}\right)_{\#}\left(b l_{\sigma}(b)\right)=\left(p_{1}\right)_{\#}\left(\left(z, r_{b}, s_{b}\right) l_{\sigma}\left(z, r_{b}, s_{b}\right)\right) \\
& =\left(r_{b}, s_{b}\right)+\left(|z|_{x},|z|_{y}\right)+\left(r_{b}, s_{b}\right)=\left(|z|_{x}+2 r_{b},|z|_{y}+2 s_{b}\right) .
\end{aligned}
$$

Segue que $|z|_{x}$ e $|z|_{y}$ são pares, pois por hipótese $m_{2}$ e $n_{2}$ são pares. Temos também que

$$
\begin{aligned}
a b l_{\sigma}(a) & =\left(w, m_{1}, n_{1}\right)\left(z, r_{b}, s_{b}\right)\left(x y^{-1} w\left(x^{-1}, y^{-1}\right) y x^{-1},|w|_{x}+m_{1},|w|_{y}+n_{1}\right) \\
& =\left(w z x y^{-1} w\left(x^{-1}, y^{-1}\right) y x^{-1}, 2 m_{1}+|w|_{x}+r_{b}, 2 n_{1}+|w|_{y}+s_{b}\right) .
\end{aligned}
$$

Pela condição $(i) a b l_{\sigma}(a)=b$, segue que $|w|_{x}=-2 m_{1},|w|_{y}=-2 n_{1}$ e como por hipótese $\left(m_{1}, n_{1}\right) \neq(0,0)$, segue também que $w$ é uma palavra não trivial. Ainda por $(i)$ temos que

$$
\begin{aligned}
w z x y^{-1} w\left(x^{-1}, y^{-1}\right) y x^{-1} & =z \\
z^{-1} w z x y^{-1} w\left(x^{-1}, y^{-1}\right) y x^{-1} & =\mathbb{1} \\
y x^{-1} z^{-1} w z x y^{-1} w\left(x^{-1}, y^{-1}\right) & =\mathbb{1} \\
\left(y x^{-1} z^{-1}\right) w\left(y x^{-1} z^{-1}\right)^{-1} & =w\left(x^{-1}, y^{-1}\right)^{-1} .
\end{aligned}
$$

Assim, pelo Lema 2.3.8, segue que $\left|y x^{-1} z^{-1}\right|_{x}=-1-|z|_{x}$ ou $\left|y x^{-1} z^{-1}\right|_{y}=1-|z|_{y}$ é par, ou equivalentemente, $|z|_{x}$ ou $|z|_{y}$ é ímpar. Mas isto é um absurdo, pois já mostramos que $|z|_{x}$ e $|z|_{y}$ são pares. Portanto, $\alpha$ tem a propriedade de Borsuk-Ulam.

Decorre imediatamente do Teorema 1.4.4 item 2., da Observação 2.1.1 e das Proposições 2.3.2, 2.3.3, 2.3.4, 2.3.5 e 2.3.9 o seguinte resultado de classificação:

Teorema 2.3.10. Seja $\tau_{2}: \mathbb{T}^{2} \rightarrow \mathbb{T}^{2}$ a involução livre de pontos fixos definida por $\tau_{2}(x, y)=\left(1-x, y+\frac{1}{2}\right)$. Seja $\beta \in\left[\mathbb{T}^{2}, \mathbb{T}^{2}\right]$ uma classe de homotopia livre e seguindo a notação da Observação 2.1.1, seja $h_{\beta}=\Delta(\beta)$. Então $\beta$ tem a propriedade de Borsuk-Ulam com respeito a $\tau_{2}$ se, e somente se, o homomorfismo

$$
\begin{array}{cccc}
h_{\beta}: \pi_{1}\left(\mathbb{T}^{2}\right)=\mathbb{Z} \oplus \mathbb{Z} & \longrightarrow & \mathbb{Z} \oplus \mathbb{Z}=\pi_{1}\left(\mathbb{T}^{2}\right) \\
(1,0) & \longmapsto & \left(m_{1}, n_{1}\right) \\
(0,1) & \longmapsto & \left(m_{2}, n_{2}\right)
\end{array}
$$

é tal que $\left(m_{1}, n_{1}\right) \neq(0,0)$ e $m_{2}$ e $n_{2}$ são pares. 


\section{Capítulo 3}

\section{$\mathrm{O}$ caso $\left(\mathbb{K}^{2}, \tau ; \mathbb{K}^{2}\right)$}

\subsection{Introdução}

Neste capítulo nós vamos estudar a propriedade de Borsuk-Ulam para classes de homotopia de funções tendo como domínio e contra-domínio a garrafa de Klein.

Pelo Teorema 1.3.5, a menos de equivalên-

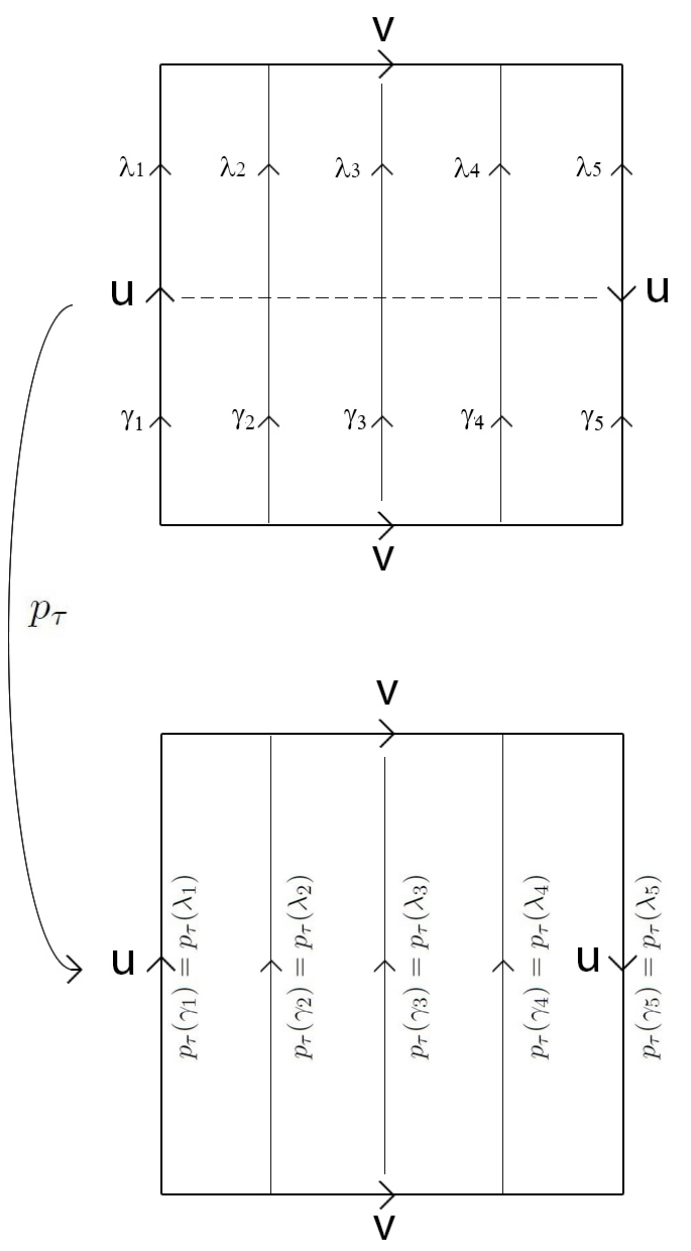

Figura 3.1: recobrimento $p_{\tau}$. cia, só existe uma involução em $\mathbb{K}^{2}$. Utilizando a notação da Seção 1.5, consideremos a involução livre de pontos fixos $\tau: \mathbb{K}^{2} \rightarrow \mathbb{K}^{2}$ definida por

$$
\tau(x, y)=\left(x, y+\frac{1}{2}\right) .
$$

O espaço de órbitas $\mathbb{K}_{\tau}^{2}$ é homeomorfo a $\mathbb{K}^{2}$. A Figura 3.1 nos mostra como é a aplicação de recobrimento $p_{\tau}: \mathbb{K}^{2} \rightarrow \mathbb{K}^{2}$. Assim, utilizando a notação da Seção 1.3 , nós temos os homorfismos induzidos por $p_{\tau}$ definidos do seguinte modo:

$$
\begin{aligned}
& \left(p_{\tau}\right)_{\#}: \quad \pi_{1}\left(\mathbb{K}^{2}\right)=\mathbb{Z} \rtimes \mathbb{Z} \longrightarrow \pi_{1}\left(\mathbb{K}^{2}\right) \\
& (1,0) \quad \longmapsto \quad(2,0) \\
& (0,1) \quad \longmapsto \quad(0,1) \\
& \theta_{\tau}: \quad \pi_{1}\left(\mathbb{K}^{2}\right)=\mathbb{Z} \rtimes \mathbb{Z} \quad \longrightarrow \quad \mathbb{Z}_{2} \\
& (1,0) \quad \longmapsto \quad \overline{1} \\
& (0,1) \quad \longmapsto \quad \overline{0} \text {. }
\end{aligned}
$$

Na Seção 3.2, nós mostraremos um resultado sobre conjugação e potência de elementos em $\mathbb{Z} \rtimes \mathbb{Z}$ (Proposição 3.2.1).

Na Seção 3.3, nós faremos uma completa descrição algébrica do conjunto $\left[\mathbb{K}^{2}, \mathbb{K}^{2}\right]$ (Teorema 3.3.1).

Na Seção 3.4, nós de fato classificaremos os elementos do conjunto $\left[\mathbb{K}^{2}, \mathbb{K}^{2}\right]$ que têm a propriedade de Borsuk-Ulam com respeito a $\tau$ (Teorema 3.4.5). Nesta seção, nós usaremos os fatos estabelecidos no Apêndice B sobre o grupo de tranças da garrafa de Klein. Em especial, usaremos a Observação B.0.18 e o Lema B.0.19, o qual transcrevemos aqui na seguinte observação: 
Observação 3.1.1 (Presentação de $P_{2}\left(\mathbb{K}^{2}\right)$ ). A menos de isomorfismo, $P_{2}\left(\mathbb{K}^{2}\right)$ se escreve na forma $F(u, v) \rtimes_{\theta}(\mathbb{Z} \rtimes \mathbb{Z})$, sendo $F(u, v)$ o grupo livre gerado por $\{u, v\}$ e $\theta: \mathbb{Z} \rtimes \mathbb{Z} \rightarrow \operatorname{Aut}(F(u, v))$ definida do seguinte modo:

$$
\begin{aligned}
& \theta(m, n):\left\{\begin{array}{ll}
u & \longmapsto B^{m-\delta_{n}} u^{\varepsilon_{n}} B^{-m+\delta_{n}} \\
v & \longmapsto B^{m} v u^{-2 m} B^{-m+\delta_{n}} \\
B & \longmapsto B^{\varepsilon_{n}}
\end{array} \quad \delta_{n}=\left\{\begin{array}{l}
0, \text { se } \text { n é par } \\
1, \text { se n é ímpar }
\end{array}\right.\right. \\
& B=u v u v^{-1} .
\end{aligned}
$$

Com respeito a esta escrita, temos que:

- $\left(p_{1}\right)_{\#}: P_{2}\left(\mathbb{K}^{2}\right) \rightarrow \pi_{1}\left(\mathbb{K}^{2}\right)=\mathbb{Z} \rtimes \mathbb{Z}$ é a projeção de $F(u, v) \rtimes_{\theta}(\mathbb{Z} \rtimes \mathbb{Z})$ em $\mathbb{Z} \rtimes \mathbb{Z}$, isto é, $\left(p_{1}\right)_{\#}(w ; r, s)=(r, s)$;

- Existe um elemento $\sigma \in B_{2}\left(\mathbb{K}^{2}\right)-P_{2}\left(\mathbb{K}^{2}\right)$ tal que $\sigma^{2}=(B ; 0,0)$;

- O homomorfismo $l_{\sigma}: P_{2}\left(\mathbb{K}^{2}\right) \rightarrow P_{2}\left(\mathbb{K}^{2}\right)$ definido por $l_{\sigma}(b)=\sigma b \sigma^{-1}$ para todo $b \in P_{2}\left(\mathbb{T}^{2}\right)$, tem os seguintes valores:

$$
\begin{array}{ll}
l_{\sigma}(u ; 0,0)=\left(B u^{-1} B^{-1} ; 1,0\right) & l_{\sigma}(B ; 0,0)=(B ; 0,0) \\
l_{\sigma}\left(u^{r} ; 0,0\right)=\left(\left(B u^{-1}\right)^{r} B^{-r} ; r, 0\right) & l_{\sigma}(\mathbb{1} ; m, 0)=(\mathbb{1} ; m, 0) \\
l_{\sigma}(v ; 0,0)=\left(v^{-1} B ; 0,1\right) & l_{\sigma}(\mathbb{1} ; 0, n)=\left(B^{\delta_{n}} ; 0, n\right) \\
l_{\sigma}\left(v^{s} ; 0,0\right)=\left((u v)^{-s}(u B)^{\delta_{s}} ; 0, s\right) &
\end{array}
$$

sendo $r, s \in \mathbb{Z}$ e o simbolo $\mathbb{1}$ denota o elemento neutro de $F(u, v)$.

Observação 3.1.2. Se $t \in B_{2}\left(\mathbb{K}^{2}\right)-P_{2}\left(\mathbb{K}^{2}\right)$, ou seja, $t$ é uma trança não pura, então $t=\sigma a$, sendo $a=\sigma^{-1} t \in P_{2}\left(\mathbb{K}^{2}\right)$. Notemos que tal escrita é única, pois se $\sigma a_{1}=\sigma a_{2}$, então $a_{1}=a_{2}$.

\subsection{Conjugação e potência em $\mathbb{Z} \rtimes \mathbb{Z}$}

Afim de facilitar certos cálculos quando estivermos trabalhando com o grupo $\mathbb{Z} \rtimes \mathbb{Z}$, vamos mostrar como computar conjugação e potências neste grupo. Utilizaremos a notação estabelecida na Observação 3.1.1.

Lembremos que como conjunto, $\mathbb{Z} \rtimes \mathbb{Z}$ é o produto cartesiano de duas cópias de $\mathbb{Z}$. Dados dois elementos $\left(r_{1}, s_{1}\right)$ e $\left(r_{2}, s_{2}\right)$, o produto destes elementos é definido do seguinte modo:

$$
\left(r_{1}, s_{1}\right)\left(r_{2}, s_{2}\right)=\left(r_{1}+\varepsilon_{s_{1}} r_{2}, s_{1}+s_{2}\right) .
$$

Temos que o elemento neutro é $(0,0)$. Dado um elemento $(r, s) \in \mathbb{Z} \rtimes \mathbb{Z}$, seu inverso é definido do seguinte modo:

$$
(r, s)^{-1}=\left(\varepsilon_{s+1} r,-s\right) .
$$

Proposição 3.2.1. Dados dois elementos $(r, s)$ e $(a, b)$ pertencentes a $\mathbb{Z} \rtimes \mathbb{Z}$ et um número inteiro, valem as seguintes fórmulas:

(1) $(a, b)(r, s)(a, b)^{-1}=\left(a+\varepsilon_{s+1} a+\varepsilon_{b} r, s\right)$;

(2) $(r, s)^{t}=\left\{\begin{array}{l}(t r, t s), \text { se s é par; } \\ \left(\delta_{t} r, t s\right), \text { se s é ímpar. }\end{array}\right.$ 
Demonstração. Começamos mostrando a fórmula (1). Temos que

$$
\begin{aligned}
(a, b)(r, s)(a, b)^{-1} & =\left(a+\varepsilon_{b} r, b+s\right)\left(\varepsilon_{b+1} a,-b\right) \\
& =\left(a+\varepsilon_{b} r+\varepsilon_{b+s} \varepsilon_{b+1} a, b+s-b\right) \\
& =\left(a+\varepsilon_{s+1} a+\varepsilon_{b} r, s\right) .
\end{aligned}
$$

Agora vamos computar $(r, s)^{t}$. Primeiro suponhamos $s$ par. Notemos que neste caso, o produto em $\mathbb{Z} \rtimes \mathbb{Z}$ se comporta de forma idêntica ao produto em $\mathbb{Z} \oplus \mathbb{Z}$, e assim temos que

$$
(r, s)^{t}=(t r, t s) .
$$

Suponhamos $s$ ímpar e $t$ par. Seja $k \in \mathbb{Z}$ tal que $t=2 k$. Assim temos

$$
(r, s)^{t}=(r, s)^{2 k}=\left((r, s)^{2}\right)^{k}=((r, s)(r, s))^{k}=(0,2 s)^{k}=(0,2 k s)=(0, t s)=\left(\delta_{t} r, t s\right) .
$$

Agora suponhamos $s$ ímpar e $t$ ímpar. Seja $k \in \mathbb{Z}$ tal que $t=2 k+1$. Já sabemos que $(r, s)^{2 k}=(0,2 k s)$. Assim, temos

$$
(r, s)^{t}=(r, s)^{2 k+1}=(r, s)^{2 k}(r, s)=(0,2 k s)(r, s)=(r, 2 k s+s)=(r,(2 k+1) s)=(r, t s)=\left(\delta_{t} r, t s\right) .
$$

\subsection{O conjunto $\left[\mathbb{K}^{2}, \mathbb{K}^{2}\right]$}

Nesta seção, vamos dar uma completa caracterização algébrica do conjunto $\left[\mathbb{K}^{2}, \mathbb{K}^{2}\right]$.

Lembremos que pelo Teorema 1.2.1, nós temos o seguinte diagrama comutativo, sendo que as flechas horizontais são bijeções e as flechas verticais são sobrejeções:

$$
\begin{array}{r}
{\left[\mathbb{K}^{2}, * ; \mathbb{K}^{2}, \mathbb{K}^{2}\right] \stackrel{\Gamma_{\mathbb{K}^{2}, \mathbb{K}^{2}}}{\longrightarrow} \operatorname{Hom}\left(\pi_{1}\left(\mathbb{K}^{2}\right), \pi_{1}\left(\mathbb{K}^{2}\right)\right)} \\
\Lambda_{\mathbb{K}^{2}, \mathbb{K}^{2}} \downarrow \\
\downarrow \Upsilon_{\mathbb{K}^{2}, \mathbb{K}^{2}} \\
{\left[\mathbb{K}^{2}, \mathbb{K}^{2}\right] \stackrel{\Delta_{\mathbb{K}^{2}, \mathbb{K}^{2}}}{\longrightarrow} \frac{\operatorname{Hom}\left(\pi_{1}\left(\mathbb{K}^{2}\right), \pi_{1}\left(\mathbb{K}^{2}\right)\right)}{\sim} .}
\end{array}
$$

Para simplificar a notação, em todo este capítulo, denotaremos as funções $\Gamma_{\mathbb{K}^{2}, \mathbb{K}^{2}}$, $\Lambda_{\mathbb{K}^{2}, \mathbb{K}^{2}}, \Delta_{\mathbb{K}^{2}, \mathbb{K}^{2}}$ e $\Upsilon_{\mathbb{K}^{2}, \mathbb{K}^{2}}$ simplesmente por $\Gamma, \Lambda, \Delta$ e $\Upsilon$, respectivamente.

Lembremos também que de acordo com a Seção 1.5, nós temos a seguinte identificação:

$$
\operatorname{Hom}\left(\pi_{1}\left(\mathbb{K}^{2}\right), \pi_{1}\left(\mathbb{K}^{2}\right)\right)=\operatorname{Hom}(\mathbb{Z} \rtimes \mathbb{Z}, \mathbb{Z} \rtimes \mathbb{Z}) .
$$

Segundo o artigo [18, Lema 3.1], se $h \in \operatorname{Hom}(\mathbb{Z} \rtimes \mathbb{Z}, \mathbb{Z} \rtimes \mathbb{Z})$, então $h$ pode ser de dois tipos, que são os seguintes:

Tipo A:

Tipo B:

$$
h:\left\{\begin{array}{l}
(1,0) \mapsto\left(r_{1}, 0\right) \\
(0,1) \mapsto\left(r_{2}, 2 s+1\right)
\end{array}\right.
$$

$$
h:\left\{\begin{array}{l}
(1,0) \mapsto(0,0) \\
(0,1) \mapsto\left(r_{2}, 2 s\right)
\end{array}\right.
$$

sendo $r_{1}, r_{2}, s \in \mathbb{Z}$.

Vamos conjugar estes homomorfismos por $(a, b) \in \mathbb{Z} \rtimes \mathbb{Z}$.

Primeiramente, suponhamos $h: \pi_{1}\left(\mathbb{K}^{2}\right) \rightarrow \pi_{1}\left(\mathbb{K}^{2}\right)$ do tipo A. Temos que 


$$
h \sim\left\{\begin{array}{l}
(a, b)\left(r_{1}, 0\right)(a, b)^{-1}=\left(\varepsilon_{b} r_{1}, 0\right) \\
(a, b)\left(r_{2}, 2 s+1\right)(a, b)^{-1}=\left(2 a+\varepsilon_{b} r_{2}, 2 s+1\right) .
\end{array}\right.
$$

Se $r_{1} \geq 0$ e $r_{2}$ é par, tomamos $a=-\frac{r_{2}}{2}$ e $b=0$. Temos que $h \sim\left\{\begin{array}{l}(1,0) \mapsto\left(r_{1}, 0\right) \\ (0,1) \mapsto(0,2 s+1) .\end{array}\right.$

Se $r_{1}<0$ e $r_{2}$ é par, tomamos $a=\frac{r_{2}}{2}$ e $b=1$. Temos que $h \sim\left\{\begin{array}{l}(1,0) \mapsto\left(-r_{1}, 0\right) \\ (0,1) \mapsto(0,2 s+1) .\end{array}\right.$

Se $r_{1} \geq 0$ e $r_{2}$ é ímpar, tomamos $a=\frac{-r_{2}+1}{2}$ e $b=0$. Temos que $h \sim\left\{\begin{array}{l}(1,0) \mapsto\left(r_{1}, 0\right) \\ (0,1) \mapsto(1,2 s+1) .\end{array}\right.$

Se $r_{1}<0$ e $r_{2}$ é ímpar, tomamos $a=\frac{r_{2}+1}{2}$ e $b=1$. Temos que $h \sim\left\{\begin{array}{l}(1,0) \mapsto\left(-r_{1}, 0\right) \\ (0,1) \mapsto(1,2 s+1) .\end{array}\right.$

Agora, suponhamos $h: \pi_{1}\left(\mathbb{K}^{2}\right) \rightarrow \pi_{1}\left(\mathbb{K}^{2}\right)$ do tipo B. Temos que

$$
h \sim\left\{\begin{array}{l}
(1,0) \mapsto(a, b)(0,0)(a, b)^{-1}=(0,0) \\
(1,0) \mapsto(a, b)\left(r_{2}, 2 s\right)(a, b)^{-1}=\left(\varepsilon_{b} r_{2}, 2 s\right) .
\end{array}\right.
$$

Tomamos $b=1$ e concluímos que

$$
h \sim\left\{\begin{array}{l}
(1,0) \mapsto(0,0) \\
(0,1) \mapsto\left(-r_{2}, 2 k\right) .
\end{array}\right.
$$

Usando a definição de produto do grupo $\mathbb{Z} \rtimes \mathbb{Z}$, é fácil ver que se temos dois homorfismos $h_{1}, h_{2}: \mathbb{Z} \rtimes \mathbb{Z} \rightarrow \mathbb{Z} \rtimes \mathbb{Z}$ tais que $h_{1}$ é um conjugado de $h_{2}$ por algum elemento de $\mathbb{Z} \rtimes \mathbb{Z}$ e

$$
h_{1}:\left\{\begin{array}{l}
(1,0) \mapsto\left(r_{1}, s_{1}\right) \\
(0,1) \mapsto\left(r_{2}, s_{2}\right)
\end{array} \quad \text { e } \quad h_{2}:\left\{\begin{array}{l}
(1,0) \mapsto\left(r_{1}^{\prime}, s_{1}^{\prime}\right) \\
(0,1) \mapsto\left(r_{2}^{\prime}, s_{2}^{\prime}\right),
\end{array}\right.\right.
$$

sendo $\left(r_{1}, s_{1}\right),\left(r_{2}, s_{2}\right),\left(r_{1}^{\prime}, s_{1}^{\prime}\right),\left(r_{2}^{\prime}, s_{2}^{\prime}\right) \in \mathbb{Z} \rtimes \mathbb{Z}$, então $s_{1}=s_{1}^{\prime}$ e $s_{2}=s_{2}^{\prime}$.

Utilizando o diagrama comutativo (3.2) e a identificação (3.3), nós podemos resumir as informações até aqui encontradas no seguinte resultado:

Teorema 3.3.1. O conjunto $\left[\mathbb{K}^{2}, \mathbb{K}^{2}\right]$ está em bijeção com as classes de conjugação de homomorfismos $h: \pi_{1}\left(\mathbb{K}^{2}\right) \rightarrow \pi_{1}\left(\mathbb{K}^{2}\right)$ dos seguintes tipos:

\section{Tipo 1:}

$h:\left\{\begin{aligned}(1,0) & \mapsto(r, 0) \\ (0,1) & \mapsto(i, 2 s+1),\end{aligned}\right.$

para $i \in\{0,1\}, r, s \in \mathbb{Z}, r \geq 0$.
Tipo 2:

$h:\left\{\begin{array}{l}(1,0) \mapsto(0,0) \\ (0,1) \mapsto(r, 2 s),\end{array}\right.$

para $r, s \in \mathbb{Z}, r \geq 0$.

\subsection{Classificação das classes de homotopia com a propriedade de Borsuk-Ulam}

Nesta seção nós vamos classificar os elementos do conjunto $\left[\mathbb{K}^{2}, \mathbb{K}^{2}\right]$ em que vale a propriedade de Borsuk-Ulam com respeito a involução $\tau$.

Para cada classe de homotopia pontuada $\alpha \in\left[\mathbb{K}^{2}, * ; \mathbb{K}^{2}, *\right]$, seguindo a notação do diagrama (3.2) e da identificação (3.3), seja $h_{\alpha}=\Gamma(\alpha): \mathbb{Z} \rtimes \mathbb{Z} \rightarrow \mathbb{Z} \rtimes \mathbb{Z}$. 
No próximo resultado, nós vamos resolver o problema de Borsuk-Ulam para metade das classes de homotopia pontuada que estão em bijeção, conforme o Teorema 3.3.1, com homomorfismos do Tipo 1.

Proposição 3.4.1. Seja $\alpha \in\left[\mathbb{K}^{2}, * ; \mathbb{K}^{2}, *\right]$ e suponhamos que

$$
h_{\alpha}(1,0)=(r, 0) \quad e \quad h_{\alpha}(0,1)=(i, 2 s+1)
$$

para algum $i \in\{0,1\}$ e $r, s \in \mathbb{Z}$, com $r$ não negativo e ímpar. Então $\alpha$ não tem a propriedade de Borsuk-Ulam com respeito a $\tau$.

Demonstração. Consideremos o homomorfismo $\psi: \mathbb{Z} \rtimes \mathbb{Z} \rightarrow \mathbb{Z} \rtimes \mathbb{Z}$, o qual é definido nos geradores por

$$
\psi(1,0)=(r, 0) \quad \text { e } \quad \psi(0,1)=(2 i, 2 s+1),
$$

sendo $i \in\{0,1\}$ e $r, s \in \mathbb{Z}$, com $r$ ímpar. Usando os valores dos homomorfismos $\left(p_{\tau}\right)_{\#}$ e $\theta_{\tau}$, os quais estão definidos em (3.1), vamos mostrar que o seguinte diagrama é comutativo:

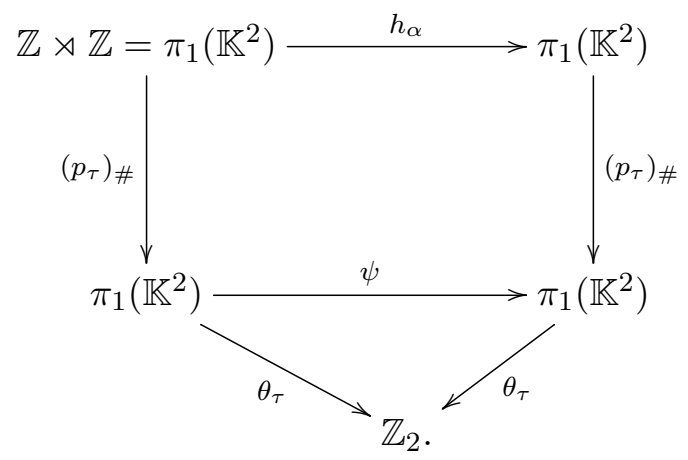

Temos que

$$
\begin{aligned}
\left(\psi \circ\left(p_{\tau}\right)_{\#}\right)(1,0) & =\psi(2,0)=\psi(1,0)^{2}=(r, 0)^{2}=(2 r, 0) \\
& =(2,0)^{r}=\left(p_{\tau}\right)_{\#}(1,0)^{r}=\left(p_{\tau}\right)_{\#}(r, 0)=\left(\left(p_{\tau}\right)_{\#} \circ h_{\alpha}\right)(1,0) \\
\left(\psi \circ\left(p_{\tau}\right)_{\#}\right)(0,1) & =\psi(0,1)=(2 i, 2 s+1)=(2,0)^{i}(0,1)^{2 s+1} \\
& =\left(p_{\tau}\right)_{\#}(1,0)^{i}\left(p_{\tau}\right)_{\#}(0,1)^{2 s+1}=\left(p_{\tau}\right)_{\#}(i, 2 s+1)=\left(\left(p_{\tau}\right)_{\#} \circ h_{\alpha}\right)(0,1)
\end{aligned}
$$

e

$$
\begin{aligned}
& \left(\theta_{\tau} \circ \psi\right)(1,0)=\theta_{\tau}(r, 0)=\theta_{\tau}\left((1,0)^{r}\right)=r \theta_{\tau}(1,0)=r \overline{1}=\overline{1}=\theta_{\tau}(1,0), \\
& \begin{aligned}
\left(\theta_{\tau} \circ \psi\right)(0,1) & =\theta_{\tau}(2 i, 2 s+1)=\theta_{\tau}\left((1,0)^{2 i}(0,1)^{2 s+1}\right) \\
& =2 i \theta_{\tau}(1,0)+(2 s+1) \theta_{\tau}(0,1)=2 i \overline{1}+(2 s+1) \overline{0}=\overline{0}=\theta_{\tau}(0,1) .
\end{aligned}
\end{aligned}
$$

Pelo Lema 1.4.1 segue que $\alpha$ não tem a propriedade de Borsuk-Ulam.

Antes de fazermos a classificação da outra metade de classes de homotopia pontuada que estão em bijeção com homomorfismos do Tipo 1, vamos estabelecer um critério algébrico, utilizando grupos de tranças, equivalente a $\alpha$ não ter a propriedade de Borsuk-Ulam. Utilizaremos fortemente a Observação 3.1.1.

Proposição 3.4.2. Uma classe de homotopia $\alpha \in\left[\mathbb{K}^{2}, * ; \mathbb{K}^{2}, *\right]$ não tem a propriedade de BorsukUlam com respeito a $\tau$ se, e somente se, existem tranças puras $a, b \in P_{2}\left(\mathbb{K}^{2}\right)$ tais que: 

(i) $l_{\sigma}(a) l_{\sigma}(b) \sigma^{2} a=b$;
(ii) $h_{\alpha}(1,0)=\left(p_{1}\right)_{\#}\left(l_{\sigma}(a) a\right)$;
(iii) $h_{\alpha}(0,1)=\left(p_{1}\right)_{\#}(b)$.

Demonstração. Seja $\alpha$ uma classe de homotopia pontuada que não tem a propriedade de BorsukUlam e vamos mostrar que existem tranças $a, b \in P_{2}\left(\mathbb{K}^{2}\right)$ tais que são verdadeiras as condições $(i)$, (ii) e (iii). Pelo Lema Fundamental 1.4.3, nós temos o seguinte diagrama comutativo:

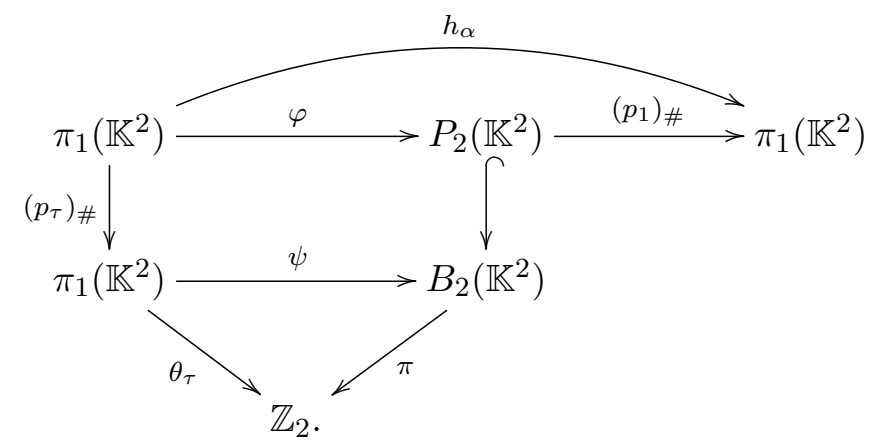

Por (3.1), como $\theta_{\tau}(1,0)=\overline{1}$ e $\theta_{\tau}(0,1)=\overline{0}$, então pela comutatividade do diagrama $(3.4), \psi(1,0)$ deve ser uma trança não pura e $\psi(0,1)$ deve ser um trança pura. Logo, pela Observação 3.1.2, existem tranças puras $a, b \in P_{2}\left(\mathbb{T}^{2}\right)$ tais que

(1) $\psi(1,0)=\sigma a$;

(2) $\psi(0,1)=b$.

Lembremos que em $\pi_{1}\left(\mathbb{K}^{2}\right)$ vale a relação $[(1,0),(0,1)]^{\prime}=(0,0)$. Logo, temos que

$$
1=[\psi(1,0), \psi(0,1)]^{\prime}=[\sigma a, b]^{\prime}=\sigma a b \sigma a b^{-1}=\sigma a \sigma^{-1} \sigma b \sigma^{-1} \sigma^{2} a b^{-1}=l_{\sigma}(a) l_{\sigma}(b) \sigma^{2} a b^{-1} .
$$

Portanto, vale

(i) $l_{\sigma}(a) l_{\sigma}(b) \sigma^{2} a=b$.

Novamente por $(3.1)$, como $\left(p_{\tau}\right)_{\#}(1,0)=(2,0)$ e $\left(p_{\tau}\right)_{\#}(0,1)=(0,1)$, segue de $(3.4)$ que valem as seguintes igualdades para o homomorfismo $\varphi$ :

(3) $\varphi(1,0)=\left(\psi \circ\left(p_{\tau}\right)_{\#}\right)(1,0)=\psi(2,0)=\psi(1,0)^{2}=(\sigma a)^{2}=\sigma a \sigma^{-1} \sigma^{2} a=l_{\sigma}(a) \sigma^{2} a$;

(4) $\varphi(0,1)=\left(\psi \circ\left(p_{\tau}\right)_{\#}\right)(0,1)=\psi(0,1)=b$.

Novamente por (3.4) e pela Observação 3.1.1, valem as seguintes igualdades para o homomorfismo $h_{\alpha}$ :

(ii) $h_{\alpha}(1,0)=\left(\left(p_{1}\right)_{\#} \circ \varphi\right)(1,0)=\left(p_{1}\right)_{\#}\left(l_{\sigma}(a) \sigma^{2} a\right)=\left(p_{1}\right)_{\#}\left(l_{\sigma}(a)(B ; 0,0) a\right)=\left(p_{1}\right)_{\#}\left(l_{\sigma}(a) a\right)$;

(iii) $h_{\alpha}(0,1)=\left(\left(p_{1}\right)_{\#} \circ \varphi\right)(b)=\left(p_{1}\right)_{\#}(b)$.

Reciprocamente, suponhamos que existam tranças $a, b \in P_{2}\left(\mathbb{T}^{2}\right)$ e valham $(i)$, $(i i)$ e $(i i i)$. Utilizando a Observação 3.1.2, definimos

$$
\begin{aligned}
\varphi: \pi_{1}\left(\mathbb{K}^{2}\right) & \longrightarrow P_{2}\left(\mathbb{K}^{2}\right) \\
(1,0) & \longmapsto l_{\sigma}(a) \sigma^{2} a \\
(0,1) & \longmapsto b
\end{aligned}
$$

e

$$
\begin{aligned}
\psi: \pi_{1}\left(\mathbb{K}^{2}\right) & \longrightarrow B_{2}\left(\mathbb{K}^{2}\right) \\
(1,0) & \longmapsto \sigma a \\
(0,1) & \longmapsto b .
\end{aligned}
$$


Mostremos que de fato $\varphi$ e $\psi$ definem homomorfismos. Pela Observação 3.1.1, temos

$$
\begin{aligned}
{[\varphi(1,0), \varphi(0,1)]^{\prime} } & =\left[l_{\sigma}(a) \sigma^{2} a, b\right]^{\prime}=\sigma a \sigma^{-1} \sigma^{2} a b \sigma a \sigma^{-1} \sigma^{2} a b^{-1}=\sigma a \sigma a \sigma^{-1} \sigma b \sigma^{-1} \sigma^{2} a \sigma^{-1} \sigma^{2} a b^{-1} \\
& =l_{\sigma}\left(a l_{\sigma}(a) l_{\sigma}(b) \sigma^{2} a\right) \sigma^{2} a b^{-1} \stackrel{(i)}{=} l_{\sigma}(a b) \sigma^{2} a b^{-1}=l_{\sigma}(a) l_{\sigma}(b) \sigma^{2} a b^{-1} \stackrel{(i)}{=} b b^{-1}=1
\end{aligned}
$$

e

$$
[\psi(1,0), \psi(0,1)]^{\prime}=[\sigma a, b]^{\prime}=\sigma a b \sigma a b^{-1}=\sigma a \sigma^{-1} \sigma b \sigma^{-1} \sigma^{2} a b^{-1}=l_{\sigma}(a) l_{\sigma}(b) \sigma^{2} a b^{-1} \stackrel{(i)}{=} b b^{-1}=1 .
$$

Vamos mostrar que temos um diagrama comutativo como em (3.4), donde seguirá pelo Lema Fundamental 1.4.3 que $\alpha$ não tem a propriedade de Borsuk-Ulam. Utilizaremos as Observações 3.1.1 e 3.1.2 e também os valores dos homomorfismos $\left(p_{\tau}\right)_{\#}$ e $\theta_{\tau}$, os quais estão definidos em (3.1). Temos

$$
\begin{aligned}
& \left(\left(p_{1}\right)_{\#} \circ \varphi\right)(1,0)=\left(p_{1}\right)_{\#}\left(l_{\sigma}(a) \sigma^{2} a\right)=\left(p_{1}\right)_{\#}\left(l_{\sigma}(a)(B ; 0,0) a\right)=\left(p_{1}\right)_{\#}\left(l_{\sigma}(a) a\right) \stackrel{(i i)}{=} h_{\alpha}(1,0), \\
& \left(\left(p_{1}\right)_{\#} \circ \varphi\right)(0,1)=\left(p_{1}\right)_{\#}(b) \stackrel{(i i i)}{=} h_{\alpha}(0,1), \\
& \left(\psi \circ\left(p_{\tau}\right)_{\#}\right)(1,0)=\psi(2,0)=\psi(1,0)^{2}=(\sigma a)^{2}=\sigma a \sigma^{-1} \sigma^{2} a=l_{\sigma}(a) \sigma^{2} a=\varphi(1,0), \\
& \left(\psi \circ\left(p_{\tau}\right)_{\#}\right)(0,1)=\psi(1,0)=b=\varphi(1,0) \\
& \mathrm{e} \\
& (\pi \circ \psi)(1,0)=\pi(\sigma a)=\overline{1}=\theta_{\tau_{1}}(1,0), \\
& (\pi \circ \psi)(0,1)=\pi(b)=\overline{0}=\theta_{\tau_{1}}(0,1) .
\end{aligned}
$$

e

Proposição 3.4.3. Seja $\alpha \in\left[\mathbb{K}^{2}, * ; \mathbb{K}^{2}, *\right]$ e suponhamos que

$$
h_{\alpha}(1,0)=(r, 0) \quad e \quad h_{\alpha}(0,1)=(i, 2 s+1)
$$

para algum $i \in\{0,1\}$ e $r, s \in \mathbb{Z}$, com $r$ não negativo e par. Então a não tem a propriedade de Borsuk-Ulam com respeito a $\tau$.

Demonstração. Seja $m \in \mathbb{Z}$ tal que $r=2 m$. Consideremos os seguintes elementos de $P_{2}\left(\mathbb{K}^{2}\right)$ :

$$
a=(\mathbb{1} ; m, 0) \quad \text { e } \quad b=(B ; i, 2 s+1) .
$$

Vamos mostrar que as tranças $a$ e $b$, juntamente com o homomorfismo $h_{\alpha}$ satisfazem os itens (i), (ii) e (iii) da Proposição 3.4.2, donde seguirá que $\alpha$ não tem a propriedade de Borsuk-Ulam. Utilizaremos a todo instante a Observação 3.1.1. Primeiramente temos que

$$
\begin{aligned}
l_{\sigma}(a) l_{\sigma}(b) \sigma^{2} a & =l_{\sigma}(\mathbb{1} ; m, 0) l_{\sigma}(B ; i, 2 s+1)(B ; 0,0)(\mathbb{1} ; m, 0) \\
& =(\mathbb{1} ; m, 0) l_{\sigma}(B ; i, 0) l_{\sigma}(\mathbb{1} ; 0,2 s+1)(B ; m, 0) \\
& =(\mathbb{1} ; m, 0)(B ; i, 0)(B ; 0,2 s+1)(B ; m, 0) \\
& =(\theta(m, 0)(B) ; m+i, 0)(B \theta(0,2 s+1)(B) ;-m, 2 s+1) \\
& =(B ; m+i, 0)\left(B B^{-1} ;-m, 2 s+1\right) \\
& =(B ; i, 2 s+1)=b
\end{aligned}
$$

e portanto, vale $(i)$. Agora temos que

$\left(p_{1}\right)_{\#}\left(l_{\sigma}(a) a\right)=\left(p_{1}\right)_{\#}\left(l_{\sigma}(\mathbb{1} ; m, 0)(\mathbb{1} ; m, 0)\right)=\left(p_{1}\right)_{\#}((\mathbb{1} ; m, 0)(\mathbb{1} ; m, 0))=(2 m, 0)=(r, 0)=h_{\alpha}(1,0)$ 
e

$\left(p_{1}\right)_{\#}(b)=\left(p_{1}\right)_{\#}(B ; i, 2 s+1)=(i, 2 s+1)=h_{\alpha}(0,1)$,

e portanto, valem $(i i)$ e (iii), o que encerra a demonstração.

No próximo resultado, nós vamos classificar, em relação a propriedade de Borsuk-Ulam, as classes de homotopia pontuada que estão em bijeção, conforme o Teorema 3.3.1, com homomorfismos do Tipo 2.

Proposição 3.4.4. Seja $\alpha \in\left[\mathbb{K}^{2}, * ; \mathbb{K}^{2}, *\right]$ e suponhamos que

$$
h_{\alpha}(1,0)=(r, 0) \quad e \quad h_{\alpha}(0,1)=(r, 2 s)
$$

para algum $r, s \in \mathbb{Z}$, com $r \geq 0$. Então $\alpha$ tem a propriedade de Borsuk-Ulam.

Demonstração. Vamos fazer a prova por contradição. Suponhamos então que $\alpha$ não tem a propriedade de Borsuk-Ulam. Por definição, existe uma função $f:\left(\mathbb{K}^{2}, *\right) \rightarrow\left(\mathbb{K}^{2}, *\right)$ que representa $\alpha$ tal que para todo $x \in \mathbb{K}^{2}$ vale

$$
f(\tau(x)) \neq f(x) .
$$

Lembremos que pelo Teorema 1.2.1, temos que $f_{\#}=h_{\alpha}$. Consideremos a involução $\tau_{2}: \mathbb{T}^{2} \rightarrow \mathbb{T}^{2}$ como definida no Capítulo 2. Lembremos que a partir desta involução, temos um recobrimento duplo $p_{\tau_{2}}: \mathbb{T}^{2} \rightarrow \mathbb{K}^{2}$ e que o homomorfismo induzido $\left(p_{\tau_{2}}\right)_{\#}: \pi_{1}\left(\mathbb{T}^{2}\right)=\mathbb{Z} \oplus \mathbb{Z} \rightarrow \mathbb{Z} \rtimes \mathbb{Z}=\pi_{1}\left(\mathbb{K}^{2}\right)$ tem os seguintes valores nos geradores, conforme (2.2):

$$
\left(p_{\tau_{2}}\right)_{\#}(1,0)=(1,0) \quad \text { e } \quad\left(p_{\tau_{2}}\right)_{\#}(0,1)=(0,2) .
$$

É fácil ver que $\operatorname{Im}\left(f_{\#}\right) \subset \operatorname{Im}\left(\left(p_{\tau_{2}}\right)_{\#}\right)$. Portanto, existe um levantamento da função $f$ pelo recobrimento $p_{\tau_{2}}$, isto é, existe $\widetilde{f}:\left(\mathbb{K}^{2}, *\right) \rightarrow\left(\mathbb{T}^{2}, *\right)$ tal que é comutativo o seguinte diagrama:

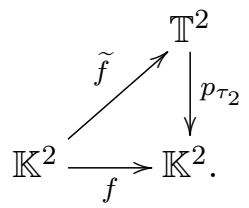

Pela condição (3.5) e pela comutatividade do diagrama (3.6) segue que para todo $x \in \mathbb{K}^{2}$ devemos ter que $\widetilde{f}(\tau(x)) \neq \widetilde{f}(x)$. Pela Definição 0.0.1, segue que a tripla $\left(\mathbb{K}^{2}, \tau ; \mathbb{T}^{2}\right)$ não tem a propriedade de Borsuk-Ulam, o que é um absurdo de acordo com o Teorema 1.3.7. Portanto, $\alpha$ tem a propriedade de Borsuk-Ulam.

Pelo Teorema 1.4.4, item 2, segue diretamente das Proposições 3.4.1, 3.4.3 e 3.4.4 o seguinte resultado de classificação:

Teorema 3.4.5. Seja $\tau: \mathbb{K}^{2} \rightarrow \mathbb{K}^{2}$ a involução livre de pontos fixos definida por $\tau(x, y)=\left(x, y+\frac{1}{2}\right)$. Consideremos a familia de homomorfismos listadas no Teorema 3.3.1. Uma classe de homotopia $\beta \in\left[\mathbb{K}^{2}, \mathbb{K}^{2}\right]$ tem a propriedade de Borsuk-Ulam com respeito a $\tau$ se, e somente se, $\Delta(\beta)$ é a classe de conjugação de um homomorfismo do tipo 2. 


\section{Capítulo 4}

\section{$\mathrm{O}$ caso $\left(\mathbb{T}^{2}, \tau ; \mathbb{K}^{2}\right)$, com $\mathbb{T}_{\tau}^{2}=\mathbb{T}^{2}$}

\subsection{Introdução}

Neste capítulo nós vamos estudar a propriedade de Borsuk-Ulam para classes de homotopia de funções tendo como domínio o Toro e contra-domínio a garrafa de Klein. Mais especificamente, nós vamos classificar os elementos do conjunto $\left[\mathbb{T}^{2}, \mathbb{K}^{2}\right]$ que têm a propriedade de Borsuk-Ulam com respeito a involução $\tau_{1}: \mathbb{T}^{2} \rightarrow \mathbb{T}^{2}$, dada por $\tau_{1}(x, y)=\left(x+\frac{1}{2}, y\right)$ (Teorema 4.4.2). Lembremos que no Capítulo 2, nós mostramos que a principal característica desta involução é que o espaço de órbitas $\mathbb{T}_{\tau_{1}}^{2}$ é homeomorfo a $\mathbb{T}^{2}$.

Na Seção 4.2, nós faremos uma completa descrição algébrica do conjunto $\left[\mathbb{T}^{2}, \mathbb{K}^{2}\right]$.

No processo de classificação dos elementos do conjunto $\left[\mathbb{T}^{2}, \mathbb{K}^{2}\right]$ que têm a propriedade de BorsukUlam, nós usaremos alguns resultados sobre o subgrupo normal gerado por $\sigma^{2} \in P_{2}\left(\mathbb{K}^{2}\right)$. Por este motivo, na Seção 4.3 nós enunciaremos e demonstraremos tais resultados, os quais serão usados nas seções seguintes.

Na Seção 4.4, nós de fato classificaremos os elementos do conjunto $\left[\mathbb{T}^{2}, \mathbb{K}^{2}\right]$ que têm a propriedade de Borsuk-Ulam com respeito a $\tau_{1}$.

\subsection{O conjunto $\left[\mathbb{T}^{2}, \mathbb{K}^{2}\right]$}

Nesta seção, nós vamos fornecer uma completa caracterização algébrica do conjunto $\left[\mathbb{T}^{2}, \mathbb{K}^{2}\right]$.

Lembremos que pelo Teorema 1.2.1, nós temos o seguinte diagrama comutativo, sendo que as flechas horizontais são bijeções e as flechas verticais são sobrejeções:

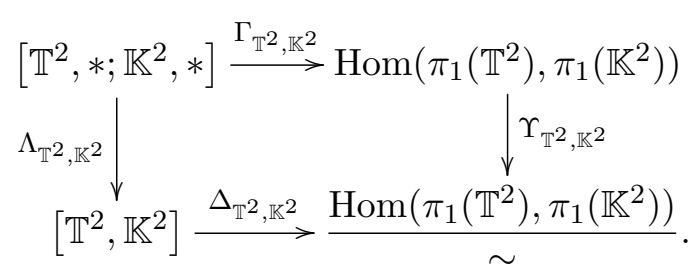

Para simplificar a notação, em todo este capítulo, denotaremos as funções $\Gamma_{\mathbb{T}^{2}, \mathbb{K}^{2}}$, $\Lambda_{\mathbb{T}^{2}, \mathbb{K}^{2}}, \Delta_{\mathbb{T}^{2}, \mathbb{K}^{2}}$ e $\Upsilon_{\mathbb{T}^{2}, \mathbb{K}^{2}}$ simplesmente por $\Gamma, \Lambda, \Delta$ e $\Upsilon$, respectivamente.

Lembremos que de acordo com a notação estabelecida na Seção 1.5, nós temos a seguinte identificação:

$$
\operatorname{Hom}\left(\pi_{1}\left(\mathbb{T}^{2}\right), \pi_{1}\left(\mathbb{K}^{2}\right)\right)=\operatorname{Hom}(\mathbb{Z} \oplus \mathbb{Z}, \mathbb{Z} \rtimes \mathbb{Z}) .
$$

Por [19, Lema 2.1], são quatro os tipos de homomorfismos $h \in \operatorname{Hom}(\mathbb{Z} \oplus \mathbb{Z}, \mathbb{Z} \rtimes \mathbb{Z})$, conforme a seguinte descrição: 
Tipo A:

$$
h:\left\{\begin{array}{l}
(1,0) \mapsto\left(r, 2 s_{1}+1\right) \\
(0,1) \mapsto\left(0,2 s_{2}\right)
\end{array}\right.
$$

Tipo B:

$$
h:\left\{\begin{array}{l}
(1,0) \mapsto\left(r, 2 s_{1}+1\right) \\
(0,1) \mapsto\left(r, 2 s_{2}+1\right)
\end{array}\right.
$$

Tipo C:

$$
h:\left\{\begin{array}{l}
(1,0) \mapsto\left(0,2 s_{1}\right) \\
(0,1) \mapsto\left(r, 2 s_{2}+1\right)
\end{array}\right.
$$

Tipo D:

$$
h:\left\{\begin{array}{l}
(1,0) \mapsto\left(r_{1}, 2 s_{1}\right) \\
(0,1) \mapsto\left(r_{2}, 2 s_{2}\right)
\end{array}\right.
$$

sendo $r, r_{1}, r_{2}, s_{1}, s_{2} \in \mathbb{Z}$.

Vamos conjugar estes homomorfismos por $(a, b) \in \mathbb{Z} \rtimes \mathbb{Z}$. Utilizaremos a notação da Seção 1.2 e o resultado da Proposição 3.2.1.

Suponhamos $h: \pi_{1}\left(\mathbb{T}^{2}\right) \rightarrow \pi_{1}\left(\mathbb{K}^{2}\right)$ do tipo $A$. Temos que

$$
h \sim\left\{\begin{array}{l}
(1,0) \mapsto(a, b)\left(r, 2 s_{1}+1\right)(a, b)^{-1}=\left(2 a+\varepsilon_{b} r, 2 s_{1}+1\right) \\
(0,1) \mapsto(a, b)\left(0,2 s_{2}\right)(a, b)^{-1}=\left(0,2 s_{2}\right) .
\end{array}\right.
$$

Se $r$ é par, tomamos $a=\frac{r}{2}$ e $b=1$. Temos que $h \sim\left\{\begin{array}{l}(1,0) \mapsto\left(0,2 s_{1}+1\right) \\ (0,1) \mapsto\left(0,2 s_{2}\right) .\end{array}\right.$

Se $r$ é ímpar, tomamos $a=\frac{r+1}{2}$ e $b=1$. Temos que $h \sim\left\{\begin{array}{l}(1,0) \mapsto\left(1,2 s_{1}+1\right) \\ (0,1) \mapsto\left(0,2 s_{2}\right) .\end{array}\right.$

Suponhamos $h: \pi_{1}\left(\mathbb{T}^{2}\right) \rightarrow \pi_{1}\left(\mathbb{K}^{2}\right)$ do tipo B. Temos que

$$
h \sim\left\{\begin{array}{l}
(1,0) \mapsto(a, b)\left(r, 2 s_{1}+1\right)(a, b)^{-1}=\left(2 a+\varepsilon_{b} r, 2 s_{1}+1\right) \\
(0,1) \mapsto(a, b)\left(r, 2 s_{2}+1\right)(a, b)^{-1}=\left(2 a+\varepsilon_{b} r, 2 s_{2}+1\right) .
\end{array}\right.
$$

Se $r$ é par, tomamos $a=\frac{r}{2}$ e $b=1$. Temos que $h \sim\left\{\begin{array}{l}(1,0) \mapsto\left(0,2 s_{1}+1\right) \\ (0,1) \mapsto\left(0,2 s_{2}+1\right) .\end{array}\right.$

Se $r$ é ímpar, tomamos $a=\frac{r+1}{2}$ e $b=1$. Temos que $h \sim\left\{\begin{array}{l}(1,0) \mapsto\left(1,2 s_{1}+1\right) \\ (0,1) \mapsto\left(1,2 s_{2}+1\right) .\end{array}\right.$

Suponhamos $h: \pi_{1}\left(\mathbb{T}^{2}\right) \rightarrow \pi_{1}\left(\mathbb{K}^{2}\right)$ do tipo $C$. Temos que

$$
h \sim\left\{\begin{array}{l}
(1,0) \mapsto(a, b)\left(0,2 s_{1}\right)(a, b)^{-1}=\left(0,2 s_{1}\right) \\
(0,1) \mapsto(a, b)\left(r, 2 s_{2}+1\right)(a, b)^{-1}=\left(2 a+\varepsilon_{b} r, 2 s_{2}+1\right) .
\end{array}\right.
$$

Se $r$ é par, tomamos $a=\frac{r}{2}$ e $b=1$. Temos que $h \sim\left\{\begin{array}{l}(1,0) \mapsto\left(0,2 s_{1}\right) \\ (0,1) \mapsto\left(0,2 s_{2}+1\right) .\end{array}\right.$

Se $r$ é ímpar, tomamos $a=\frac{r+1}{2}$ e $b=1$. Temos que $h \sim\left\{\begin{array}{l}(1,0) \mapsto\left(0,2 s_{1}\right) \\ (0,1) \mapsto\left(1,2 s_{2}+1\right) .\end{array}\right.$

Suponhamos $h: \pi_{1}\left(\mathbb{T}^{2}\right) \rightarrow \pi_{1}\left(\mathbb{K}^{2}\right)$ do tipo D. Temos que

$$
h \sim\left\{\begin{array}{l}
(1,0) \mapsto(a, b)\left(r_{1}, 2 s_{1}\right)(a, b)^{-1}=\left(\varepsilon_{b} r_{1}, 2 s_{1}\right) \\
(0,1) \mapsto(a, b)\left(r_{2}, 2 s_{2}\right)(a, b)^{-1}=\left(\varepsilon_{b} r_{2}, 2 s_{2}\right) .
\end{array}\right.
$$


Tomamos $a=0$ e $b=1$. Temos que $h \sim\left\{\begin{array}{l}(1,0) \mapsto\left(-r_{1}, 2 s_{1}\right) \\ (0,1) \mapsto\left(-r_{2}, 2 s_{2}\right) .\end{array}\right.$

Utilizando o diagrama comutativo (4.1) e a identificação (4.2), nós podemos resumir as informações até aqui encontradas no seguinte resultado:

Teorema 4.2.1. O conjunto $\left[\mathbb{T}^{2}, \mathbb{K}^{2}\right]$ está em bijeção com as classes de conjugação de homomorfismos $h: \pi_{1}\left(\mathbb{T}^{2}\right) \rightarrow \pi_{1}\left(\mathbb{K}^{2}\right)$ dos seguintes tipos:

Tipo 1:

$h:\left\{\begin{array}{l}(1,0) \mapsto\left(i, 2 s_{1}+1\right) \\ (0,1) \mapsto\left(0,2 s_{2}\right)\end{array}\right.$

para $i \in\{0,1\}$ e $s_{1}, s_{2} \in \mathbb{Z}$.

Tipo 3:

$h:\left\{\begin{array}{l}(1,0) \mapsto\left(0,2 s_{1}\right) \\ (0,1) \mapsto\left(i, 2 s_{2}+1\right),\end{array}\right.$

para $i \in\{0,1\}$ e $s_{1}, s_{2} \in \mathbb{Z}$.
Tipo 2:

$h:\left\{\begin{array}{l}(1,0) \mapsto\left(i, 2 s_{1}+1\right) \\ (0,1) \mapsto\left(i, 2 s_{2}+1\right),\end{array}\right.$

para $i \in\{0,1\}$ e $s_{1}, s_{2} \in \mathbb{Z}$.

Tipo 4:

$h:\left\{\begin{array}{l}(1,0) \mapsto\left(r_{1}, 2 s_{1}\right) \\ (0,1) \mapsto\left(r_{2}, 2 s_{2}\right),\end{array}\right.$

para $r_{1}, r_{2}, s_{1}, s_{2} \in \mathbb{Z}, r_{1} \geq 0$.

\subsection{Algumas propriedades de $\overline{\left\langle\sigma^{2}\right\rangle}$}

O objetivo desta seção é mostrar alguns resultados e propriedades relativos ao grupo de tranças puras da garrafa de Klein e mais detalhadamente sobre o subgrupo normal gerado por $\sigma^{2} \in P_{2}\left(\mathbb{K}^{2}\right)$.

Utilizaremos os fatos e notações estabelecidos na Seção 3.2, a qual trata sobre o grupo $\mathbb{Z} \rtimes \mathbb{Z}$. Assim como no Capítulo 3, neste capítulo nós também vamos utilizar os resultados obtidos no Apêndice B, em especial a Observação B.0.18 e o Lema B.0.19, os quais nós transcrevemos na seguinte observação:

Observação 4.3.1 (Presentação de $P_{2}\left(\mathbb{K}^{2}\right)$ ). A menos de isomorfismo, $P_{2}\left(\mathbb{K}^{2}\right)$ se escreve na forma $F(u, v) \rtimes_{\theta}(\mathbb{Z} \rtimes \mathbb{Z})$, sendo $F(u, v)$ o grupo livre gerado por $\{u, v\}$ e $\theta: \mathbb{Z} \rtimes \mathbb{Z} \rightarrow \operatorname{Aut}(F(u, v))$ definida do seguinte modo:

$$
\begin{aligned}
\theta(m, n):\left\{\begin{array}{ll}
u & \longmapsto B^{m-\delta_{n}} u^{\epsilon_{n}} B^{-m+\delta_{n}} \\
v & \longmapsto B^{m} v u^{-2 m} B^{-m+\delta_{n}} \\
B & \longmapsto B^{\varepsilon_{n}}
\end{array} \delta_{n}=\left\{\begin{array}{l}
0, \text { se né par } \\
1, \text { se né impar }
\end{array}\right.\right. \\
\varepsilon_{n}=(-1)^{n}
\end{aligned}
$$

Com respeito a esta escrita, temos que:

- $\left(p_{1}\right)_{\#}: P_{2}\left(\mathbb{K}^{2}\right) \rightarrow \pi_{1}\left(\mathbb{K}^{2}\right)=\mathbb{Z} \rtimes \mathbb{Z}$ é a projeção de $F(u, v) \rtimes_{\theta}(\mathbb{Z} \rtimes \mathbb{Z})$ em $\mathbb{Z} \rtimes \mathbb{Z}$, isto é, $\left(p_{1}\right)_{\#}(w ; r, s)=(r, s)$;

- Existe um elemento $\sigma \in B_{2}\left(\mathbb{K}^{2}\right)-P_{2}\left(\mathbb{K}^{2}\right)$ tal que $\sigma^{2}=(B ; 0,0)$;

- O homomorfismo $l_{\sigma}: P_{2}\left(\mathbb{K}^{2}\right) \rightarrow P_{2}\left(\mathbb{K}^{2}\right)$ definido por $l_{\sigma}(b)=\sigma b \sigma^{-1}$ para todo $b \in P_{2}\left(\mathbb{T}^{2}\right)$, tem os seguintes valores, sendo $r, s \in \mathbb{Z}$ e o simbolo $\mathbb{1}$ denota o elemento neutro de $F(u, v)$ :

$$
\begin{array}{ll}
l_{\sigma}(u ; 0,0)=\left(B u^{-1} B^{-1} ; 1,0\right) & l_{\sigma}(B ; 0,0)=(B ; 0,0) \\
l_{\sigma}\left(u^{r} ; 0,0\right)=\left(\left(B u^{-1}\right)^{r} B^{-r} ; r, 0\right) & l_{\sigma}(\mathbb{1} ; m, 0)=(\mathbb{1} ; m, 0) \\
l_{\sigma}(v ; 0,0)=\left(v^{-1} B ; 0,1\right) & l_{\sigma}(\mathbb{1} ; 0, n)=\left(B^{\delta_{n}} ; 0, n\right) \\
l_{\sigma}\left(v^{s} ; 0,0\right)=\left((u v)^{-s}(u B)^{\delta_{s}} ; 0, s\right) &
\end{array}
$$


Observação 4.3.2. Pela Observação 4.3.1, é fácil ver que para todo $(m, n) \in \mathbb{Z} \rtimes \mathbb{Z}$, temos que os automorfismos $\theta(m, n), \theta\left(m, \delta_{n}\right): F(u, v) \rightarrow F(u, v)$ são iguais, pois $\theta(m, n)$ depende de $m$ e da paridade de $n$.

Consideremos as funções

$$
\begin{aligned}
i: F(u, v) & \longrightarrow P_{2}\left(\mathbb{K}^{2}\right) \\
w & \longmapsto(w ; 0,0)
\end{aligned} \quad \text { e } \quad p_{F}: \begin{aligned}
P_{2}\left(\mathbb{K}^{2}\right) & \longrightarrow F(u, v) \\
(w ; m, n) & \longmapsto w .
\end{aligned}
$$

Notemos que $i$ é homomorfismo, mas $p_{F}$ não é homomorfismo por causa da ação de $\theta$. Consideremos agora a função

$$
\rho: F(u, v) \rightarrow F(u, v), \quad \text { definida pela composição } \quad \rho=p_{F} \circ l_{\sigma} \circ i .
$$

Consideremos ainda o homomorfismo

$$
\begin{aligned}
g: F(u, v) & \longrightarrow \mathbb{Z} \rtimes \mathbb{Z} \\
u & \longmapsto(1,0) \\
v & \longmapsto(0,1)
\end{aligned}
$$

o qual é definido nos geradores e estendido por linearidade.

Notemos que pela Observação 4.3.1, o seguinte diagrama é comutativo:

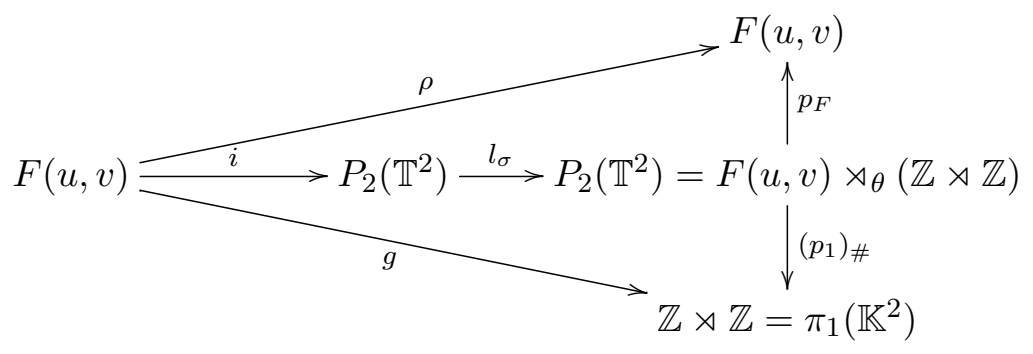

e portanto, para cada $w \in F(u, v)$, temos que

$$
l_{\sigma}(w ; 0,0)=(\rho(w) ; g(w)) .
$$

Se $w, z \in P_{2}\left(\mathbb{K}^{2}\right)$, então temos que

$$
\begin{aligned}
\rho(w z) & =\left(p_{F} \circ l_{\sigma}\right)(w z ; 0,0)=p_{F}\left(l_{\sigma}(w ; 0,0) l_{\sigma}(z ; 0,0)\right) \\
& =p_{F}((\rho(w) ; g(w))(\rho(z) ; g(z))) \\
& =p_{F}((\rho(w) \theta(g(w))(\rho(z)) ; g(w) g(z))) \\
& =\rho(w) \theta(g(w))(\rho(z))
\end{aligned}
$$

ou seja, a função $\rho: F(u, v) \rightarrow F(u, v)$ não é um homomorfismo. Mas notemos que se $w, z \in \operatorname{ker} g$, então $\rho(w z)=\rho(w) \rho(z)$.

No Apêndice B, Teorema B.0.21, está demonstrado que o núcleo do homomorfismo $g$ é um grupo livre e que

$$
\operatorname{ker} g=\left\langle B_{s, r}:=v^{s} u^{r} B u^{-r} v^{-s} \mid-\right\rangle_{s, r \in \mathbb{Z}}, \quad \text { sendo } \quad B=u v u v^{-1} \in F(u, v) .
$$

Para cada $w \in F(u, v)$ e para cada $(m, n) \in \mathbb{Z} \rtimes \mathbb{Z}$, pela Observação 4.3.1, temos que

$$
\theta(m, n)\left(w B w^{-1}\right)=\theta(m, n)(w) \theta(m, n)(B) \theta(m, n)(w)^{-1}=\theta(m, n)(w) B^{\delta_{n}} \theta(m, n)(w)^{-1}
$$

e como $g(B)=g\left(u v u v^{-1}\right)=[(1,0),(0,1)]^{\prime}=(0,0)$, então $\theta(m, n)\left(w B w^{-1}\right) \in \operatorname{ker} g$. 
Temos também que

$$
\begin{aligned}
l_{\sigma}\left(w B w^{-1} ; 0,0\right) & =l_{\sigma}(w ; 0,0) l_{\sigma}(B ; 0,0) l_{\sigma}(w ; 0,0)^{-1} \stackrel{(4.6)}{=}(\rho(w) ; g(w))(B ; 0,0)(\rho(w) ; g(w))^{-1} \\
& =(\rho(w) \theta(g(w))(B) ; g(w))\left(\theta\left(g(w)^{-1}\right)\left(\rho(w)^{-1}\right) ; g(w)^{-1}\right) \\
& =\left(\rho(w) \theta(g(w))(B) \theta(g(w))\left(\theta\left(g(w)^{-1}\right)\left(\rho(w)^{-1}\right)\right) ; g(w) g(w)^{-1}\right) \\
& =\left(\rho(w) \theta(g(w))(B) \theta\left(g(w) g(w)^{-1}\right)\left(\rho(w)^{-1}\right) ; 0,0\right) \\
& =\left(\rho(w) \theta(g(w))(B) \rho(w)^{-1} ; 0,0\right)
\end{aligned}
$$

e portanto, como $\theta(g(w))(B) \in$ ker $g$, de acordo com a Observação 4.3.1, segue pela comutatividade do diagrama (4.5) e pela igualdade (4.9) que

$$
\rho\left(w B w^{-1}\right)=\rho(w) \theta(g(w))(B) \rho(w)^{-1} \in \operatorname{ker} g .
$$

Logo, por (4.7), (4.8) e (4.10), nós temos por restrição os seguintes homomorfismos bem definidos:

$$
\theta: \mathbb{Z} \rtimes \mathbb{Z} \rightarrow \operatorname{Aut}(\operatorname{ker} g) \quad \text { e } \quad \rho: \operatorname{ker} g \rightarrow \operatorname{ker} g .
$$

Seja $H=i(\operatorname{ker} g) \subset P_{2}\left(\mathbb{K}^{2}\right)$. Denotemos por $\overline{\left\langle\sigma^{2}\right\rangle}$ o fecho normal de $\sigma^{2}=(B ; 0,0)$ em $P_{2}\left(\mathbb{K}^{2}\right)$, isto é, $\overline{\left\langle\sigma^{2}\right\rangle}$ é igual a interseção de todos os subgrupos normais de $P_{2}\left(\mathbb{K}^{2}\right)$ que contém o elemento $\sigma^{2}$.

Como $i: \operatorname{ker} g \rightarrow F(u, v) \rtimes_{\theta}(\mathbb{Z} \rtimes \mathbb{Z})=P_{2}\left(\mathbb{K}^{2}\right)$ é injetora, então $H$ é um grupo livre e por (4.7), temos que

$$
H=\left\langle\left(B_{s, r} ; 0,0\right) \mid \_\right\rangle_{s, r \in \mathbb{Z}} \cdot
$$

Notemos que $\left(B_{s, r} ; 0,0\right)=\left(v^{s} u^{r} B u^{-r} v^{-s} ; 0,0\right)=\left(v^{s} u^{r} ; 0,0\right)(B ; 0,0)\left(v^{s} u^{r} ; 0,0\right)^{-1} \in \overline{\left\langle\sigma^{2}\right\rangle}$, e portanto, $H \subset \overline{\left\langle\sigma^{2}\right\rangle}$.

Agora, se $w \in F(u, v)$ e $q \in \mathbb{Z} \rtimes \mathbb{Z}$ (e portanto $\left.(w ; q) \in P_{2}\left(\mathbb{K}^{2}\right)\right)$, então

$$
\begin{aligned}
(w ; q)\left(B_{s, r} ; 0,0\right)(w ; q)^{-1} & =\left(w \theta(q)\left(B_{s, r}\right) ; q\right)\left(\theta\left(q^{-1}\right)\left(w^{-1}\right) ; q^{-1}\right) \\
& =\left(w \theta(q)\left(B_{s, r}\right) \theta(q)\left(\theta\left(q^{-1}\right)\left(w^{-1}\right)\right) ; q q^{-1}\right) \\
& =\left(w \theta(q)\left(B_{s, r}\right) w^{-1} ; 0,0\right) .
\end{aligned}
$$

Como $\theta(q)\left(B_{s, r}\right) \in \operatorname{ker} g \triangleleft F(u, v)$, então $w \theta(q)\left(B_{s, r}\right) w^{-1} \in \operatorname{ker} g$. Logo, por (4.11), nós temos que $(w ; q)\left(B_{s, r} ; 0,0\right)(w ; q)^{-1} \in i(\operatorname{ker} g)=H$, ou seja, nós mostramos que $H$ é um subgrupo normal de $P_{2}\left(\mathbb{K}^{2}\right)$. Como $\sigma^{2}=(B ; 0,0)=\left(B_{0,0} ; 0,0\right) \in H$, então $\overline{\left\langle\sigma^{2}\right\rangle} \subset H$. Portanto, $\overline{\left\langle\sigma^{2}\right\rangle}=H$.

Como resumo das informações obtidas até aqui, nós podemos enunciar a seguinte observação:

Observação 4.3.3. O subgrupo $\overline{\left\langle\sigma^{2}\right\rangle}$ de $P_{2}\left(\mathbb{K}^{2}\right)=F(u, v) \rtimes_{\theta}(\mathbb{Z} \rtimes \mathbb{Z})$ é livre. Mais ainda, a seguinte função é uma bijeção:

$$
\begin{array}{cl}
\operatorname{ker} g=\left\langle B_{s, r}:=v^{s} u^{r} B u^{-r} v^{-s} \mid-\right\rangle_{s, r \in \mathbb{Z}} & \longrightarrow \overline{\left\langle\sigma^{2}\right\rangle} \\
B_{s, r} & \longmapsto\left(B_{s, r} ; 0,0\right),
\end{array}
$$

sendo $g: F(u, v) \rightarrow F(u, v)$ como definido em (4.4) e $B=u v u v^{-1} \in F(u, v)$. Portanto, nós podemos identificar de maneira natural $\operatorname{ker} g$ como $\overline{\left\langle\sigma^{2}\right\rangle}$. Usando esta identificaçâo, por restrição, nós temos um homomorfismo $\theta: \mathbb{Z} \rtimes \mathbb{Z} \rightarrow \operatorname{Aut}\left(\overline{\left\langle\sigma^{2}\right\rangle}\right)$. Temos também um homomorfismo $\rho: \overline{\left\langle\sigma^{2}\right\rangle} \rightarrow \overline{\left\langle\sigma^{2}\right\rangle}$ (que como função está definido em (4.3)) tal que o seguinte diagrama é comutativo: 


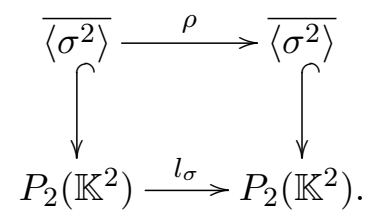

Proposição 4.3.4 (Forma normal de $F(u, v)$ ). Dado um elemento $w \in F(u, v)$, se $g(w)=(r, s)$, então existe um único elemento $x \in \overline{\left\langle\sigma^{2}\right\rangle}$ tal que $w$ se escreve como

$$
w=u^{r} v^{s} x
$$

Demonstração. Seja $x=v^{-s} u^{-r} w$. Então $w=u^{r} v^{s} x$. Temos que

$$
\begin{aligned}
g(x) & =g\left(v^{-s} u^{-r} w\right)=g(v)^{-s} g(u)^{-r} g(w)=(0,1)^{-s}(1,0)^{-r}(r, s) \\
& =(0,-s)(-r, 0)(r, s)=(0,-s)(0, s)=(0,0),
\end{aligned}
$$

e portanto, pela Observação 4.3.3, temos que $x \in \overline{\left\langle\sigma^{2}\right\rangle}$. É claro que tal elemento é único.

Como $\overline{\left\langle\sigma^{2}\right\rangle}$ é um subgrupo normal de $F(u, v)$, então para cada dupla de números inteiros $p, q \in \mathbb{Z}$, o seguinte homomorfismo está bem definido:

$$
\begin{aligned}
c_{p, q}: \overline{\left\langle\sigma^{2}\right\rangle} & \longrightarrow \overline{\left\langle\sigma^{2}\right\rangle} \\
x & \longrightarrow v^{p} u^{q} x u^{-q} v^{-p}
\end{aligned}
$$

Lema 4.3.5. Dados um elemento gerador $B_{s, r} \in \overline{\left\langle\sigma^{2}\right\rangle}$, um elemento $(m, n) \in \mathbb{Z} \rtimes \mathbb{Z}$ e $p, q \in \mathbb{Z}$, então existem $\gamma, \lambda, \eta \in \overline{\left\langle\sigma^{2}\right\rangle}$ tais que (estamos utilizando a notação estabelecida na Observação 4.3.1):

(a) $\theta(m, n)\left(B_{s, r}\right)=\gamma B_{s, \varepsilon_{n} r-2 \delta_{s} m}^{\varepsilon_{n}} \gamma^{-1}$;

(b) $\rho\left(B_{s, r}\right)=\lambda B_{-s, \varepsilon_{(s+1)}}^{\varepsilon_{s}} \lambda^{-1}$;

(c) $c_{p, q}\left(B_{s, r}\right)=\eta B_{s+p, r+\varepsilon_{s} q} \eta^{-1}$.

Demonstração. Em toda esta demonstração, usaremos as Observações 4.3.1 e 4.3.3. Usaremos também a Proposição 3.2.1. Primeiramente, temos que

$$
\begin{aligned}
\theta(m, n)\left(B_{s, r}\right)= & \theta(m, n)\left(v^{s} u^{r} B u^{-r} v^{-s}\right)=\theta(m, n)\left(v^{s} u^{r}\right) B^{\varepsilon_{n}} \theta(m, n)\left(v^{s} u^{r}\right)^{-1} \\
= & \theta(m, n)\left(v^{s} u^{r}\right)\left(u^{-\varepsilon_{n} r+2 \delta_{s} m} v^{-s} v^{s} u^{\varepsilon_{n} r-2 \delta_{s} m}\right) B^{\varepsilon_{n}} \\
& \left(u^{-\varepsilon_{n} r+2 \delta_{s} m} v^{-s} v^{s} u^{\varepsilon_{n} r-2 \delta_{s} m}\right) \theta(m, n)\left(v^{s} u^{r}\right)^{-1} \\
= & \left(\theta(m, n)\left(v^{s} u^{r}\right) u^{\varepsilon_{(n+1)} r+2 \delta_{s} m} v^{-s}\right) B_{s, \varepsilon_{n} r-2 \delta_{s} m}^{\varepsilon_{n}}\left(\theta(m, n)\left(v^{s} u^{r}\right) u^{-\varepsilon_{(n+1)} r+2 \delta_{s} m} v^{-s}\right)^{-1} .
\end{aligned}
$$

Mostremos que o elemento $\gamma=\theta(m, n)\left(v^{s} u^{r}\right) u^{\varepsilon(n+1)}{ }^{r+2 \delta_{s} m} v^{-s} \in F(u, v)$ pertence a ker $g$, o que é suficiente para que (a) fique provado. Temos que

$$
\gamma=\theta(m, n)\left(v^{s} u^{r}\right) u^{\varepsilon_{(n+1)} r+2 \delta_{s} m} v^{-s}=\left(B^{m} v u^{-2 m} B^{-m+\delta_{n}}\right)^{s}\left(B^{m-\delta_{n}} u^{\varepsilon_{n}} B^{-m+\delta_{n}}\right)^{r} u^{\varepsilon_{(n+1)}}{ }^{r+2 \delta_{s} m} v^{-s}
$$

e portanto,

$$
\begin{aligned}
g(\gamma) & =((0,1)(-2 m, 0))^{s}\left(\varepsilon_{n} r, 0\right)\left(\varepsilon_{(n+1)} r+2 \delta_{s} m, 0\right)(0,-s) \\
& =(2 m, 1)^{s}\left(\varepsilon_{n} r+\varepsilon_{(n+1)} r+2 \delta_{s} m,-s\right)=\left(2 \delta_{s} m, s\right)\left(2 \delta_{s} m,-s\right) \\
& =\left(2 \delta_{s} m+2 \delta_{s} \varepsilon_{s} m, 0\right)=(0,0) .
\end{aligned}
$$


Agora temos que

$$
\begin{aligned}
l_{\sigma}\left(v^{s} u^{r} ; 0,0\right) & =\left((u v)^{-s}(u B)^{\delta_{s}} ; 0, s\right)\left(\left(B u^{-1}\right)^{r} B^{-r} ; r, 0\right) \\
& =\left((u v)^{-s}(u B)^{\delta_{s}} \theta(0, s)\left(\left(B u^{-1}\right)^{r} B^{-r}\right) ; \varepsilon_{s} r, s\right) \\
& =\left((u v)^{-s}(u B)^{\delta_{s}}\left(B^{\varepsilon_{s}}\left(B^{-\delta_{s}} u^{\varepsilon_{s}} B^{\delta_{s}}\right)^{-1}\right)^{r} B^{-\varepsilon_{s} r} ; \varepsilon_{s} r, s\right) \\
& =\left((u v)^{-s}(u B)^{\delta_{s}}\left(B^{\varepsilon_{s}} B^{-\delta_{s}} u^{-\varepsilon_{s}} B^{\delta_{s}}\right)^{r} B^{\varepsilon_{(s+1)} r} ; \varepsilon_{s} r, s\right) \\
& =\left((u v)^{-s}(u B)^{\delta_{s}} B^{-\delta_{s}}\left(B^{\varepsilon_{s}} u^{\varepsilon_{(s+1)}}\right)^{r} B^{\delta_{s}+\varepsilon_{(s+1)}} ; \varepsilon_{s} r, s\right)
\end{aligned}
$$

e portanto, temos que

$$
\begin{aligned}
& \rho\left(B_{s, r}\right)=\rho\left(v^{s} u^{r} B u^{-r} v^{-s}\right) \stackrel{(4.10)}{=} \rho\left(v^{s} u^{r}\right) \theta\left(g\left(v^{s} u^{r}\right)\right)(B) \rho\left(v^{s} u^{r}\right)^{-1} \\
& =\left(p_{F} \circ l_{\sigma} \circ i\right)\left(v^{s} u^{r}\right) \theta((0, s)(r, 0))(B)\left(p_{F} \circ l_{\sigma} \circ i\right)\left(v^{s} u^{r}\right)^{-1} \\
& =(u v)^{-s}(u B)^{\delta_{s}} B^{-\delta_{s}}\left(B^{\varepsilon_{s}} u^{\varepsilon_{(s+1)}}\right)^{r} B^{\delta_{s}+\varepsilon_{(s+1)^{r}}} B^{\varepsilon_{s}}\left((u v)^{-s}(u B)^{\delta_{s}} B^{-\delta_{s}}\left(B^{\varepsilon_{s}} u^{\varepsilon_{(s+1)}}\right)^{r} B^{\delta_{s}+\varepsilon_{(s+1)^{r}}}\right)^{-1} \\
& =(u v)^{-s}(u B)^{\delta_{s}} B^{-\delta_{s}}\left(B^{\varepsilon_{s}} u^{\varepsilon_{(s+1)}}\right)^{r} B^{\delta_{s}+\varepsilon_{(s+1)^{r}}}\left(u^{-\varepsilon_{(s+1)^{r}}} v^{s} v^{-s} u^{\varepsilon_{(s+1)^{r}}}\right) B^{\varepsilon_{s}}\left(u^{-\varepsilon_{(s+1)} r} v^{s} v^{-s} u^{\varepsilon_{(s+1)^{r}}}\right) \\
& \left((u v)^{-s}(u B)^{\delta_{s}} B^{-\delta_{s}}\left(B^{\varepsilon_{s}} u^{\varepsilon_{(s+1)}}\right)^{r} B^{\delta_{s}+\varepsilon_{(s+1)}}\right)^{-1} \\
& =\left((u v)^{-s}(u B)^{\delta_{s}} B^{-\delta_{s}}\left(B^{\varepsilon_{s}} u^{\varepsilon_{(s+1)}}\right)^{r} B^{\delta_{s}+\varepsilon_{(s+1)} r} u^{\varepsilon_{s} r} v^{s}\right) B_{-s, \varepsilon_{(s+1)}}^{\varepsilon_{s}} \\
& \left((u v)^{-s}(u B)^{\delta_{s}} B^{-\delta_{s}}\left(B^{\varepsilon_{s}} u^{\varepsilon_{(s+1)}}\right)^{r} B^{\delta_{s}+\varepsilon_{(s+1)} r} u^{\varepsilon_{s} r} v^{s}\right)^{-1} .
\end{aligned}
$$

Mostremos que o elemento $\lambda=(u v)^{-s}(u B)^{\delta_{s}} B^{-\delta_{s}}\left(B^{\varepsilon_{s}} u^{\varepsilon_{(s+1)}}\right)^{r} B^{\delta_{s}+\varepsilon_{(s+1)} r} u^{\varepsilon_{s} r} v^{s} \in F(u, v)$ pertence a ker $g$, o que é suficiente para que (b) fique provado. Temos que

$$
\begin{aligned}
g(\lambda) & =g\left((u v)^{-s}(u B)^{\delta_{s}} B^{-\delta_{s}}\left(B^{\varepsilon_{s}} u^{\varepsilon_{(s+1)}}\right)^{r} B^{\delta_{s}+\varepsilon_{(s+1)} r} u^{\varepsilon_{s} r} v^{s}\right)=(1,1)^{-s}\left(\delta_{s}, 0\right)\left(\varepsilon_{(s+1)} r, 0\right)\left(\varepsilon_{s} r, s\right) \\
& =\left(\delta_{(-s)},-s\right)\left(\delta_{s}+\varepsilon_{(s+1)} r+\varepsilon_{s} r, s\right)=\left(\delta_{s}+\varepsilon_{(-s)} \delta_{s}, 0\right)=(0,0) .
\end{aligned}
$$

Por fim, temos que

$$
\begin{aligned}
c_{p, q}\left(B_{s, r}\right) & =v^{p} u^{q} B_{s, r} u^{-q} v^{-p}=v^{p} u^{q} v^{s} u^{r} B u^{-r} v^{-s} u^{-q} v^{-p} \\
& =v^{p} u^{q} v^{s} u^{r}\left(u^{-r-\varepsilon_{s} q} v^{-s-p} v^{s+p} u^{r+\varepsilon_{s} q}\right) B\left(u^{-r-\varepsilon_{s} q} v^{-s-p} v^{s+p} u^{r+\varepsilon_{s} q}\right) u^{-r} v^{-s} u^{-q} v^{-p} \\
& =\left(v^{p} u^{q} v^{s} u^{\varepsilon_{(s+1)} q} v^{-s-p}\right) B_{s+p, r+\varepsilon_{s} q}\left(v^{p} u^{q} v^{s} u^{\varepsilon_{(s+1)} q} v^{-s-p}\right)^{-1} .
\end{aligned}
$$

Seja $\eta=v^{p} u^{q} v^{s} u^{\varepsilon_{(s+1)} q} v^{-s-p} \in F(u, v)$ e mostremos que $\eta$ pertence a ker $g$, o que é suficiente para que (c) fique provado. Temos que

$$
\begin{aligned}
g(\eta) & =g\left(v^{p} u^{q} v^{s} u^{\varepsilon_{(s+1)} q} v^{-s-p}\right)=(0, p)(q, s)\left(\varepsilon_{(s+1)} q,-s-p\right) \\
& =(0, p)\left(q+\varepsilon_{s} \varepsilon_{(s+1)} q,-p\right)=(0, p)(0,-p)=(0,0) .
\end{aligned}
$$

Seja $\overline{\left\langle\sigma^{2}\right\rangle_{\mathrm{ab}}}$ o abelianizado do grupo $\overline{\left\langle\sigma^{2}\right\rangle}$. Vamos fazer um abuso de notação e denotaremos a imagem de um gerador $B_{s, r}$ em ${\overline{\left\langle\sigma^{2}\right\rangle}}_{\mathrm{ab}}$ também por $B_{s, r}$. Pela Observação $4.3 .3,{\overline{\left\langle\sigma^{2}\right\rangle}}_{\mathrm{ab}}$ é o grupo abeliano livre gerado pelo conjunto $\left\{B_{s, r}:=v^{s} u^{r} B u^{-r} v^{-s}\right\}$, ou seja, temos que

$$
{\overline{\left\langle\sigma^{2}\right\rangle_{\mathrm{ab}}}}_{=} \bigoplus_{s, r \in \mathbb{Z}} \mathbb{Z}\left[B_{s, r}\right] .
$$

Decorre imediatamente do Lema 4.3.5 o seguinte resultado: 
Proposição 4.3.6. Para cada $(m, n) \in \mathbb{Z} \rtimes \mathbb{Z}$ e para par $p, q \in \mathbb{Z}$, por passagem ao quociente, nós temos os homomorfismos:

$$
\begin{aligned}
& \theta(m, n)_{\mathrm{ab}}:{\overline{\left\langle\sigma^{2}\right\rangle}}_{\mathrm{ab}} \rightarrow{\overline{\left\langle\sigma^{2}\right\rangle_{\mathrm{ab}}}} ; \\
& \rho_{\mathrm{ab}}:{\overline{\left\langle\sigma^{2}\right\rangle}}_{\mathrm{ab}} \rightarrow{\overline{\left\langle\sigma^{2}\right\rangle}}_{\mathrm{ab}} ; \\
& \left(c_{p, q}\right)_{\mathrm{ab}}:{\overline{\left\langle\sigma^{2}\right\rangle}}_{\mathrm{ab}} \rightarrow{\overline{\left\langle\sigma^{2}\right\rangle}}_{\mathrm{ab}} .
\end{aligned}
$$

Para cada gerador $B_{s, r} \in{\overline{\left\langle\sigma^{2}\right\rangle}}_{\mathrm{ab}}$, temos que

$$
\begin{aligned}
& \theta(m, n)_{\mathrm{ab}}\left(B_{s, r}\right)=\left\{\begin{array}{l}
B_{s, r}, \text { se } n \text { é par e s é par; } \\
B_{s, r-2 m}, \text { se } n \text { é par e s é impar; } \\
-B_{s,-r}, \text { se } n \text { é ímpar e s é par; } \\
-B_{s,-r-2 m}, \text { se } n \text { é impar e s é impar; }
\end{array}\right. \\
& \rho_{\mathrm{ab}}\left(B_{s, r}\right)=\left\{\begin{array}{l}
B_{-s,-r}, \text { se s é par; } \\
-B_{-s, r}, \text { se s é impar } ;
\end{array}\right. \\
& \left(c_{p, q}\right)_{\mathrm{ab}}\left(B_{s, r}\right)=\left\{\begin{array}{l}
B_{s+p, r+q}, \text { se sé par } ; \\
B_{s+p, r-q}, \text { se sé impar. }
\end{array}\right.
\end{aligned}
$$

Nas próximas seções, nós teremos especial interesse em certos elementos de $\overline{\left\langle\sigma^{2}\right\rangle}$ e como eles se escrevem no abelianizado em termos da base $\left\{B_{s, r}\right\}$. Este é o conteúdo do seguinte resultado:

Proposição 4.3.7. Para cada $k, l \in \mathbb{Z}$, consideremos os seguintes elementos de $F(u, v)$ :

(i) $T_{k}=u^{k}\left(B u^{-1}\right)^{k}$;

(ii) $W_{k}=u^{k}\left(B^{-1} u\right)^{-k}$;

(iii) $I_{k}=v^{2 k}(v B)^{-2 k}$;

(iv) $O_{k, l}=\left[v^{2 k}, u^{l}\right]$;

(v) $D_{k, l}=(v B)^{2 k}\left(v u^{l} B\right)^{-2 k}$;

(vi) $J_{k, l}=v^{2 k}\left(v u^{l}\right)^{-2 k}$.

Temos que estes elementos pertencem a $\overline{\left\langle\sigma^{2}\right\rangle}$. Denotemos por $\widetilde{T}_{k}, \widetilde{W}_{k}, \widetilde{I}_{k}, \widetilde{O}_{k, l}, \widetilde{D}_{k, l}$ e $\widetilde{J}_{k, l}$ a projeção destes elementos em ${\overline{\left\langle\sigma^{2}\right\rangle}}_{\mathrm{ab}}$. Para $k=0$, temos que $\widetilde{T}_{0}=\widetilde{W}_{0}=\widetilde{I}_{0}=0$ e se $k=0$ ou $l=0$, então $\widetilde{O}_{k, l}=\widetilde{D}_{k, l}=\widetilde{J}_{k, l}=0$. Para cada $k, l>0$ valem as seguintes fórmulas:
(1) $\widetilde{T}_{k}=\sum_{i=1}^{k} B_{0, i}$
(2) $\widetilde{T}_{-k}=-\sum_{i=1}^{k} B_{0,-i+1}$;
(3) $\widetilde{W}_{k}=\sum_{i=1}^{k} B_{0, i-1}$;
(4) $\widetilde{W}_{-k}=-\sum_{i=1}^{k} B_{0,-i}$;
(5) $\widetilde{I}_{k}=-\sum_{i=1}^{k}\left(B_{2 i-1,0}+B_{2 i, 0}\right)$;
(6) $\widetilde{I}_{-k}=\sum_{i=1}^{k}\left(B_{-2 i+1,0}+B_{-2 i+2,0}\right)$; 
(11) $\widetilde{D}_{k, l}=-\sum_{i=1}^{k} \sum_{j=1}^{l} B_{2 i-1, j}$;

(13) $\widetilde{D}_{-k, l}=\sum_{i=1}^{k} \sum_{j=1}^{l} B_{-2 i+1, j}$;

(15) $\widetilde{J}_{k, l}=-\sum_{i=1}^{k} \sum_{j=1}^{l} B_{2 i-1, j-1}$;

(17) $\widetilde{J}_{-k, l}=\sum_{i=1}^{k} \sum_{j=1}^{l} B_{-2 i+1, j-1}$;

(7) $\widetilde{O}_{k, l}=\sum_{i=1}^{k} \sum_{j=1}^{l}\left(B_{2 i-1,-j}-B_{2 i-2, j-1}\right)$;

(9) $\widetilde{O}_{-k, l}=\sum_{i=1}^{k} \sum_{j=1}^{l}\left(B_{-2 i, j-1}-B_{-2 i+1,-j}\right)$;
(12) $\widetilde{D}_{k,-l}=\sum_{i=1}^{k} \sum_{j=1}^{l} B_{2 i-1,-j+1}$;

(14) $\widetilde{D}_{-k,-l}=-\sum_{i=1}^{k} \sum_{j=1}^{l} B_{-2 i+1,-j+1}$;

(16) $\widetilde{J}_{k,-l}=\sum_{i=1}^{k} \sum_{j=1}^{l} B_{2 i-1,-j}$;

(18) $\widetilde{J}_{-k,-l}=-\sum_{i=1}^{k} \sum_{j=1}^{l} B_{-2 i+1,-j}$.

(8) $\widetilde{O}_{k,-l}=\sum_{i=1}^{k} \sum_{j=1}^{l}\left(B_{2 i-2,-j}-B_{2 i-1, j-1}\right)$;

(10) $\widetilde{O}_{-k,-l}=\sum_{i=1}^{k} \sum_{j=1}^{l}\left(B_{-2 i+1, j-1}-B_{-2 i,-j}\right)$;

A prova das fórmulas de (1) a (18) da Proposição 4.3.7 consiste essencialmente em manipular adequadamente cada elemento listado no enunciado para obter uma relação recursiva e usar indução. Uma demonstração direta seria longa e entendiante. Para que fique claro qual manipulação será feita em cada elemento, nós vamos enunciar e provar 5 resultados. Deste modo, a Proposição 4.3.7 decorrerá imediatamente dos Lemas 4.3.8, 4.3.9, 4.3.10, 4.3.11 e 4.3.12.

Lema 4.3.8. Para cada $k \in \mathbb{Z}$, consideremos os elementos $T_{k}=u^{k}\left(B u^{-1}\right)^{k}, W_{k}=u^{k}\left(B^{-1} u\right)^{-k} \in$ $F(u, v)$. Se $k=0$, então $T_{k}=W_{k}=\mathbb{1}$. Se $k>0$, então valem as seguintes fórmulas:
(1) $T_{k}=\prod_{i=1}^{k} B_{0, i}$
(2) $T_{-k}=\prod_{i=1}^{k} B_{0,-i+1}^{-1}$;
(3) $W_{k}=\prod_{i=1}^{k} B_{0, i-1}$;
(4) $W_{-k}=\prod_{i=1}^{k} B_{0,-i}^{-1}$;

Demonstração. É claro que se $k=0$, então $T_{0}=W_{0}=\mathbb{1}$. Notemos que

$$
T_{1}=u B u^{-1}=B_{0,1},
$$

e portanto, a fórmula (1) do Enunciado do Lema é verdadeira para $k=1$. Suponhamos então que a fórmula é verdadeira para um certo $k \geq 1$ e vamos mostrar por indução que a fórmula é verdadeira para $k+1$. Assim, temos que

$$
\begin{aligned}
T_{k+1} & =u^{k+1}\left(B u^{-1}\right)^{-k-1}=u\left(u^{k}\left(B u^{-1}\right)\right) u^{-1} u\left(B u^{-1}\right)=u T_{k} u^{-1} T_{1} \\
& =u\left(\prod_{i=1}^{k} B_{0, i}\right) u B_{0,1}=\left(\prod_{i=1}^{k} B_{0, i+1}\right) B_{0,1}=\prod_{i=1}^{k+1} B_{0, i} .
\end{aligned}
$$

Agora, vamos usar a fórmula (1) (que já mostramos ser verdadeira) para mostrar a fórmula (2). 
Temos que

$$
\begin{aligned}
T_{-k} & =u^{-k}\left(B u^{-1}\right)^{-k}=u^{-k}\left(u^{k}\left(B u^{-1}\right)\right)^{-1} u^{k}=u^{-k} T_{k}^{-1} u^{k} \\
& =u^{-k}\left(\prod_{i=1}^{k} B_{0, i}\right)^{-1} u^{k}=\left(\prod_{i=1}^{k} B_{0,-k+i}\right)^{k}=\prod_{i=1}^{k} B_{0,-i+1}^{-1} .
\end{aligned}
$$

Notemos que

$$
W_{k}=u^{k}\left(B^{-1} u\right)^{-k}=u^{-1}\left(u^{k}\left(B u^{-1}\right)^{k}\right) u=u^{-1} T_{k} u .
$$

Assim, a fórmula (3) (resp. (4)) segue diretamente da fórmula (1) (resp. (2)), o que encerra a demonstração.

Lema 4.3.9. Para cada $k \in \mathbb{Z}$, seja $I_{k}=v^{2 k}(v B)^{-2 k} \in F(u, v)$. Se $k=0$, então $I_{k}=0$. Se $k>0$, então valem as seguintes fórmulas:

$$
\text { (1) } I_{k}=\prod_{i=1}^{k}\left(B_{2 i-1,0}^{-1} B_{2 i, 0}^{-1}\right) ; \quad \text { (2) } I_{-k}=\prod_{i=1}^{k}\left(B_{-2 i+2,0} B_{-2 i+1,0}\right) \text {. }
$$

Demonstração. É claro que se $k=0$, então $I_{0}=\mathbb{1}$. Notemos que

$$
I_{1}=v^{2}(v B)^{-2}=v^{2} B^{-1} v^{-2} v B^{-1} v^{-1}=B_{2,0}^{-1} B_{1,0}^{-1} .
$$

e portanto, a fórmula (1) do Enunciado do Lema é verdadeira para $k=1$. Suponhamos então que a fórmula é verdadeira para um certo $k \geq 1$ e vamos mostrar por indução que a fórmula é verdadeira para $k+1$. Assim, temos que

$$
\begin{aligned}
I_{k+1} & =v^{2(k+1)}(v B)^{-2(k+1)}=v^{2}\left(v^{2 k}(v B)^{-2 k}\right) v^{-2}\left(v^{2}(v B)^{-2}\right)=v^{2} I_{k} v^{-2} I_{1} \\
& =v^{2}\left(\prod_{i=1}^{k}\left(B_{2 i-1,0}^{-1} B_{2 i, 0}^{-1}\right)\right) v^{-2} B_{2,0}^{-1} B_{1,0}^{-1}=\prod_{i=1}^{k}\left(B_{2 i+1,0}^{-1} B_{2 i+2,0}^{-1}\right) B_{2,0}^{-1} B_{1,0}^{-1}=\prod_{i=1}^{k+1}\left(B_{2 i-1,0}^{-1} B_{2 i, 0}^{-1}\right) .
\end{aligned}
$$

Vamos usar a fórmula (1) (que já mostramos ser verdadeira) para mostrar a fórmula (2), o que completará a demonstração. Temos que

$$
\begin{aligned}
I_{-k} & =v^{-2 k}(v B)^{2 k}=v^{-2 k}\left(v^{2 k}(v B)^{-2 k}\right)^{-1} v^{2 k}=v^{-2 k} I_{k}^{-1} v^{2 k}=v^{-2 k}\left(\prod_{i=1}^{k}\left(B_{2 i-1,0}^{-1} B_{2 i, 0}^{-1}\right)\right)^{-1} v^{2 k} \\
& =\left(\prod_{i=1}^{k}\left(B_{-2 k+2 i-1,0}^{-1} B_{-2 k+2 i, 0}^{-1}\right)\right)^{-1}=\prod_{i=1}^{k}\left(B_{-2 i+2,0} B_{-2 i+1,0}\right) .
\end{aligned}
$$

Lema 4.3.10. Para cada $k, l \in \mathbb{Z}$, seja $D_{k, l}=(v B)^{2 k}\left(v u^{l} B\right)^{-2 k} \in F(u, v)$. Se $k=0$ ou $l=0$, então $D_{k, l}=\mathbb{1}$. Se $k, l>0$, então para $1 \leq i \leq k, 1 \leq j \leq l$ e $\varepsilon \in\{-1,1\}$, considerando $\omega=\left\{\begin{array}{l}0, \text { se } \varepsilon=-1, \\ 1, \text { se } \varepsilon=1,\end{array} \quad\right.$ valem as seguintes fórmulas:

(1) $D_{k, \varepsilon l}=\prod_{i=1}^{k}\left(I_{k-i}^{-1}\left(\prod_{j=1}^{l} B_{2 k-2 i+1, \varepsilon j-\omega+1}^{-\varepsilon}\right) I_{k-i}\right)$;

(2) $D_{-k, \varepsilon l}=I_{-k}\left(\prod_{i=1}^{k}\left(v^{-2 k} I_{k-i}^{-1} v^{2 k}\left(\prod_{j=1}^{l} B_{-2 i+1, \varepsilon j-\omega+1}^{-\varepsilon}\right) v^{-2 k} I_{k-i} v^{2 k}\right)\right)^{-1} I_{-k}^{-1}$. 
Demonstração. É claro que se $k=0$ ou $l=0$, então $D_{k, l}=\mathbb{1}$. Fixemos $k=1$ e vamos mostrar que a fórmula (1) é verdadeira para todo $l>0$. Primeiramente, para $\varepsilon=1$ temos que

$$
D_{1,1}=(v B)^{2}(v u B)^{-2}=v u v u v^{-1} v B B^{-1} u^{-1} v^{-1} B^{-1} u^{-1} v^{-1}=v u B^{-1} u^{-1} v^{-1}=B_{1,1}^{-1}
$$

e para $\varepsilon=-1$ temos

$$
D_{1,-1}=(v B)^{2}\left(v u^{-1} B\right)^{-2}=v B v B B^{-1} u v^{-1} v u^{-1} v^{-1} u^{-1} u v^{-1}=v B v^{-1}=B_{1,0} .
$$

Portanto, a fórmula (1) é verdadeira para $l=1$. Suponhamos então que a fórmula (1) é verdadeira para os números naturais até um certo $l>0$ e vamos mostrar por indução que a fórmula é verdadeira para $l+1$. Assim, para $\varepsilon=1$ temos que

$$
\begin{aligned}
D_{1, l+1} & =(v B)^{2}\left(v u^{l+1} B\right)^{-2}=(v B)^{2}\left(v u^{l} B\right)^{-2}=v u^{l} u v u v^{-1} v u^{l} B B^{-1} u^{-l-1} v^{-1} B^{-1} u^{-l-1} v^{-1} \\
& =D_{1, l} B_{1, l+1}^{-1}=\left(\prod_{j=1}^{l} B_{1, j}^{-1}\right) B_{1, l+1}^{-1}=\prod_{j=1}^{l+1} B_{1, j}^{-1}
\end{aligned}
$$

e para $\varepsilon=-1$, temos que

$$
\begin{aligned}
D_{1,-(l+1)} & =(v B)^{2}\left(v u^{-(l+1)} B\right)^{-2}=(v B)^{2}\left(v u^{-l} B\right)^{-2} v u^{-l} B v u^{-l} B B^{-1} u^{l+1} v^{-1} v u^{-1} v^{-1} u^{-1} u^{l+1} v^{-1} \\
& =D_{1,-l} B_{1,-l}=\left(\prod_{j=1}^{l} B_{1,-j+1}\right) B_{1,-l}=\prod_{j=1}^{l+1} B_{1,-j+1} .
\end{aligned}
$$

Agora, fixemos $l>0$ e vamos mostrar que a fórmula (1) é verdadeira para todo $k>0$. Notemos que para $k=1$, a fórmula está provada pela primeira parte da demonstração. Suponhamos então que a fórmula é verdadeira para os números naturais até um certo $k>0$ e vamos mostrar por indução que a fórmula é verdadeira para $k+1$. Temos que

$$
\begin{aligned}
D_{k+1, \varepsilon l}= & (v B)^{2 k+2}\left(v u^{\varepsilon l} B\right)^{-2 k-2} \\
= & \left(v^{2 k}(v B)^{-2 k}\right)^{-1} v^{2 k}(v B)^{2}\left(v u^{\varepsilon l} B\right)^{-2} v^{-2 k} v^{2 k}(v B)^{-2 k}(v B)^{2 k}\left(v u^{\varepsilon l} B\right)^{-2 k} \\
= & I_{k}^{-1} v^{2 k} D_{1, \varepsilon l} v^{-2 k} I_{k} D_{k, \varepsilon l} \\
= & I_{k}^{-1} v^{2 k}\left(\prod_{j=1}^{l} B_{1, \varepsilon l-\omega+1}^{-\varepsilon}\right) v^{-2 k} I_{k}\left(\prod_{i=1}^{k}\left(I_{k-i}^{-1}\left(\prod_{j=1}^{l} B_{2 k-2 i+1, \varepsilon j-\omega+1}^{-\varepsilon}\right) I_{k-i}\right)\right) \\
= & I_{(k+1)-1}^{-1}\left(\prod_{j=1}^{l} B_{2(k+1)-1, \varepsilon l-\omega+1}^{-\varepsilon} I_{(k+1)-1}\right) \\
& \left(\prod_{i=2}^{k+1}\left(I_{(k+1)-i}^{-1}\left(\prod_{j=1}^{l} B_{2(k+1)-2 i+1, \varepsilon j-\omega+1}^{-\varepsilon}\right) I_{(k+1)-i}\right)\right) \\
= & \prod_{i=1}^{k+1}\left(I_{(k+1)-i}^{-1}\left(\prod_{j=1}^{l} B_{2(k+1)-2 i+1, \varepsilon j-\omega+1}^{-\varepsilon}\right) I_{(k+1)-i}\right) .
\end{aligned}
$$

Vamos usar a fórmula (1) (que já mostramos ser verdadeira) para mostrar que a fórmula (2) é 
verdadeira, o que completará a demonstração. Assim, temos que

$$
\begin{aligned}
D_{-k, \varepsilon l} & =(v B)^{-2 k}\left(v u^{\varepsilon l} B\right)^{2 k}=\left(v^{-2 k}(v B)^{2 k}\right)^{-1} v^{-2 k}\left((v B)^{2 k}\left(v u^{\varepsilon l} B\right)^{-2 k}\right)^{-1} v^{2 k} v^{-2 k}(v B)^{2 k} \\
& =I_{-k}^{-1} v^{-2 k} D_{k, \varepsilon l}^{-1} v^{2 k} I_{k}=I_{-k}^{-1} v^{-2 k}\left(\prod_{i=1}^{k}\left(I_{k-i}^{-1}\left(\prod_{j=1}^{l} B_{2 k-2 i+1, \varepsilon j-\omega+1}^{-\varepsilon} I_{k-i}\right)\right)^{-1} v^{2 k} I_{k}\right. \\
& =I_{-k}\left(\prod_{i=1}^{k}\left(v^{-2 k} I_{k-i}^{-1} v^{2 k}\left(\prod_{j=1}^{l} B_{-2 i+1, \varepsilon j-\omega+1}^{-\varepsilon}\right) v^{-2 k} I_{k-i} v^{2 k}\right)\right)^{-1} I_{-k}^{-1} .
\end{aligned}
$$

Lema 4.3.11. Para cada $k, l \in \mathbb{Z}$, seja $J_{k, l}=v^{2 k}\left(v u^{l}\right)^{-2 k} \in F(u, v)$. Se $k=0$ ou $l=0$, então $J_{k, l}=$ 1. Se $k, l>0$, então para $1 \leq i \leq k, 1 \leq j \leq l$ e $\varepsilon \in\{-1,1\}$, considerando $\omega=\left\{\begin{array}{l}0, \text { se } \varepsilon=-1, \\ 1, \text { se } \varepsilon=1,\end{array}\right.$ valem as seguintes fórmulas:

$$
\text { (1) } J_{k, \varepsilon l}=\prod_{i=1}^{k} \prod_{j=1}^{l} B_{2 k-2 i+1, \varepsilon j-\omega}^{-\varepsilon} ; \quad \text { (2) } J_{-k, \varepsilon l}=\left(\prod_{i=1}^{k} \prod_{j=1}^{l} B_{-2 i+1, \varepsilon j-\omega}^{-\varepsilon}\right)^{-1} \text {. }
$$

Demonstração. É claro que se $k=0$ ou $l=0$, então $J_{k, l}=\mathbb{1}$. Antes de mostrarmos que são verdadeiras as fórmulas (1) e (2), vamos mostrar que as seguintes fórmulas são verdadeiras para todo $l>0$ :

$$
\left(1^{\prime}\right) J_{1, l}=\prod_{i=1}^{l} B_{1, j-1}^{-1} ; \quad\left(1^{\prime \prime}\right) J_{1,-l}=\prod_{i=1}^{l} B_{1,-j} .
$$

Primeiramente, temos que

$$
J_{1,1}=v^{2}(v u)^{-2}=v v u^{-1} v^{-1} u^{-1} v^{-1}=v B^{-1} v^{-1}=B_{1,0}^{-1}
$$

e

$$
J_{1,-1}=v^{2}\left(v u^{-1}\right)^{-2}=v u^{-1} u v u v^{-1} u v^{-1}=v u^{-1} B u v^{-1}=B_{1,-1}
$$

e portanto, as fórmula $\left(1^{\prime}\right)$ e $\left(1^{\prime \prime}\right)$ são verdadeiras para $l=1$. Suponhamos que as fórmulas são verdadeiras para os números naturais até um certo $l \geq 1$ e vamos mostrar por indução que as fórmulas são verdadeiras para $l+1$. Temos que

$$
\begin{aligned}
J_{1, l+1} & =v^{2}\left(v u^{l+1}\right)^{-2}=v^{2}\left(v u^{l}\right)^{-2} v u^{l} v u^{l} u^{-l} u^{-1} v^{-1} u^{-1} u^{-l} v^{-1} \\
& =J_{1, l} v u^{l} B^{-1} u^{-l} v^{-1}=\left(\prod_{j=1}^{l} B_{1, j-1}^{-1}\right) B_{1, l}^{-1}=\left(\prod_{j=1}^{l+1} B_{1, j-1}^{-1}\right)
\end{aligned}
$$

e

$$
\begin{aligned}
J_{1,-(l+1)} & =v^{2}\left(v u^{-(l+1)}\right)^{-2}=v^{2}\left(v u^{-l}\right)^{-2} v u^{-l-1} u v u^{-l} u^{l+1} v^{-1} u^{l+1} v^{-1} \\
& =J_{1,-l} v u^{-l-1} B u^{l+1} v^{-1}=\left(\prod_{i=1}^{l} B_{1,-j}\right) B_{1,-l-1}=\prod_{i=1}^{l+1} B_{1,-j} .
\end{aligned}
$$

Agora, fixemos $l>0$ e vamos mostrar que as fórmulas do enunciado do Lema são verdadeiras para cada $k>0$. Notemos que a fórmula (1) para $k=1$ decorre imediatamente das fórmulas $\left(1^{\prime}\right)$ e $\left(1^{\prime \prime}\right)$. 
Suponhamos então que a fórmula (1) é verdadeira para números naturais até um certo $k \geq 1 \mathrm{e}$ vamos mostrar por indução que a fórmula é verdadeira para $k+1$. Assim, temos que

$$
\begin{aligned}
J_{k+1, \varepsilon l} & =v^{2 k+2}\left(v u^{\varepsilon l}\right)^{-2 k-2}=v^{2}\left(v^{2 k}\left(v u^{\varepsilon l}\right)^{-2 k}\right) v^{-2}\left(v^{2}\left(v u^{\varepsilon l}\right)^{-2}\right)=v^{2} J_{k, \varepsilon l} v^{-2} J_{1, \varepsilon l} \\
& =v^{2}\left(\prod_{i=1}^{k} \prod_{j=1}^{l} B_{2 k-2 i+1, \varepsilon j-\omega}^{-\varepsilon}\right) v^{-2}\left(\prod_{j=1}^{l} B_{1, \varepsilon j-\omega}^{-\varepsilon}\right)=\prod_{i=1}^{k+1} \prod_{j=1}^{l} B_{2(k+1)-2 i+1, \varepsilon j-\omega}^{-\varepsilon} .
\end{aligned}
$$

Vamos usar a fórmula (1) (que já mostramos ser verdadeira) para mostrar que a fórmula (2) é verdadeira, o que completará a demonstração. Assim, temos que

$$
\begin{aligned}
J_{-k, \varepsilon l} & =v^{-2 k}\left(v u^{\varepsilon l}\right)^{2 k}=v^{-2 k}\left(v^{2 k}\left(v u^{\varepsilon l}\right)^{-2 k}\right)^{-1} v^{2 k}=v^{-2 k} J_{k, \varepsilon l}^{-1} v^{2 k} \\
& =v^{-2 k}\left(\prod_{i=1}^{k} \prod_{j=1}^{l} B_{2 k-2 i+1, \varepsilon j-\omega}^{-\varepsilon}\right)^{-1} v^{2 k}=\left(\prod_{i=1}^{k} \prod_{j=1}^{l} B_{-2 i+1, \varepsilon j-\omega}^{-\varepsilon}\right)^{-1} .
\end{aligned}
$$

Lema 4.3.12. Para cada $k, l \in \mathbb{Z}$, seja $O_{k, l}=\left[v^{2 k}, u^{l}\right] \in F(u, v)$. Se $k=0$ ou $l=0$, entẫo $O_{k, l}=\mathbb{1}$. Se $k, l>0$, então para $1 \leq i \leq k, 1 \leq j \leq l$ e $\varepsilon \in\{-1,1\}$, considerando $\omega=\left\{\begin{array}{l}0, \text { se } \varepsilon=-1, \\ 1, \text { se } \varepsilon=1,\end{array}\right.$ existem elementos $\eta_{i, j, \varepsilon}, \zeta_{i, j, \varepsilon}, \mu_{i, j, \varepsilon}, \nu_{i, j, \varepsilon} \in \overline{\left\langle\sigma^{2}\right\rangle}$ tais que valem as seguintes fórmulas:

(1) $O_{\varepsilon k, l}=\prod_{i=1}^{k} \prod_{j=1}^{l}\left(\eta_{i, j, \varepsilon} B_{\varepsilon(2 i-1),-j} \eta_{i, j, \varepsilon}^{-1} \zeta_{i, j, \varepsilon} B_{2(\varepsilon i-\omega), j-1}^{-1} \zeta_{i, j, \varepsilon}^{-1}\right)^{\varepsilon}$;

(2) $O_{\varepsilon k,-l}=\prod_{i=1}^{k} \prod_{j=1}^{l}\left(\mu_{i, j, \varepsilon} B_{\varepsilon(2 i-1), j-1} \mu_{i, j, \varepsilon}^{-1} \nu_{i, j, \varepsilon} B_{2(\varepsilon i-\omega+1),-j}^{-1} \zeta_{i, j,-\varepsilon}^{-1}\right)^{-\varepsilon}$;

Demonstração. É claro que se $k=0$ ou $l=0$, então $O_{k, l}=\mathbb{1}$. Antes de mostrarmos que são verdadeiras as fórmulas (1) e (2), vamos mostrar que as seguintes fórmulas são verdadeiras para todo $k>0$ :

$$
\left(1^{\prime}\right) O_{k, 1}=\prod_{i=1}^{k}\left(B_{2 i-1,-1} B_{2 i-2,0}^{-1}\right) ; \quad\left(1^{\prime \prime}\right) O_{-k, 1}=\prod_{i=1}^{k}\left(B_{-2 i, 0} B_{-2 i+1,-1}^{-1}\right) .
$$

Primeiramente, temos que

$$
O_{1,1}=v^{2} u v^{-2} u^{-1}=v u^{-1} u v u v^{-1} u v^{-1} v u^{-1} v^{-1} u^{-1}=v u^{-1} B u v^{-1} B^{-1}=B_{1,-1} B_{0,0}^{-1},
$$

e portanto, a fórmula $\left(1^{\prime}\right)$ é verdadeira para $k=1$. Suponhamos que a fórmula $\left(1^{\prime}\right)$ é verdadeira para os números naturais até um certo $k \geq 1$ e vamos mostrar por indução que a fórmula é verdadeira para $k+1$. Temos que

$$
\begin{aligned}
O_{k+1,1} & =v^{2(k+1)} u v^{-2(k+1)} u^{-1}=v^{2} v^{2 k} u v^{-2 k} u^{-1} v^{-2} v^{2} u v^{-2} u^{-1}=v^{2} O_{k, 1} v^{-2} O_{1,1} \\
& =v^{2}\left(\prod_{i=1}^{k}\left(B_{2 i-1,-1} B_{2 i-2,0}^{-1}\right)\right) v^{-2} B_{1,-1} B_{0,0}^{-1}=\prod_{i=1}^{k}\left(B_{2 i+1,-1} B_{2 i, 0}^{-1}\right) B_{1,-1} B_{0,0}^{-1} \\
& =\prod_{i=1}^{k+1}\left(B_{2 i-1,-1} B_{2 i-2,0}^{-1}\right) .
\end{aligned}
$$


Vamos usar a fórmula $\left(1^{\prime}\right)$ (que já mostramos ser verdadeira) para mostrar a fórmula ( $\left.1^{\prime \prime}\right)$. Assim, temos que

$$
\begin{aligned}
O_{-k, 1} & =v^{-2 k} u v^{2 k} u^{-1}=v^{-2 k}\left(v^{2 k} u v^{-2 k} u^{-1}\right)^{-1} v^{2 k}=v^{-2 k} O_{k, 1}^{-1} v^{2 k} \\
& =v^{-2 k}\left(\prod_{i=1}^{k}\left(B_{2 i-1,-1} B_{2 i-2,0}^{-1}\right)\right)^{-1} v^{2 k}=\left(\prod_{i=1}^{l}\left(B_{2 i-2 k-1,-1} B_{2 i-2 k-2,0}^{-1}\right)\right)^{-1} \\
& =\prod_{i=1}^{k}\left(B_{-2 i, 0} B_{-2 i+1,-1}^{-1}\right) .
\end{aligned}
$$

Agora, fixemos $k>0$ e vamos mostrar que as fórmulas do enunciado do Lema são verdadeiras para cada $l>0$. Notemos que a fórmula (1) para $l=1$ decorre imediatamente das fórmulas $\left(1^{\prime}\right)$ e $\left(1^{\prime \prime}\right)$. Para cada $\varepsilon \in\{-1,1\}$, temos que

$$
O_{\varepsilon k, l+1}=v^{2 \varepsilon k} u^{l+1} v^{-2 \varepsilon k} u^{-l-1}=v^{2 \varepsilon k} u^{l} v^{-2 \varepsilon k} u^{-l} u^{l} v^{2 \varepsilon k} u v^{-2 \varepsilon k} u^{-1} u^{-l}=O_{\varepsilon k, l} u^{l} O_{\varepsilon k, 1} u^{-l} .
$$

Suponhamos que a fórmula (1) é verdadeira para os números naturais até um certo $l \geq 1$ e vamos mostrar por indução, usando as fórmulas $\left(1^{\prime}\right)$ e $\left(1^{\prime \prime}\right)$ e a igualdade (4.13), que as fórmulas são verdadeiras para $l+1$. Pelo Lema 4.3 .5 , para cada $1 \leq i \leq k$, existem $\eta_{i, l+1, \varepsilon}, \zeta_{i, l+1, \varepsilon} \in \overline{\left\langle\sigma^{2}\right\rangle}$ tais que $u^{l} B_{\varepsilon(2 i-1),-1} u^{-l}=\eta_{i, l+1, \varepsilon} B_{\varepsilon(2 i-1),-l-1} \eta_{i, l+1, \varepsilon}^{-1}$ e $u^{l} B_{2(\varepsilon i-\omega), 0} u^{-l}=\zeta_{i, l+1, \varepsilon} B_{2(\varepsilon i-\omega), l} \zeta_{i, l+1, \varepsilon}^{-1}$. Assim, temos que

$$
\begin{aligned}
O_{\varepsilon k, l+1}= & \left(\prod_{i=1}^{k} \prod_{j=1}^{l}\left(\eta_{i, j, \varepsilon} B_{\varepsilon(2 i-1),-j} \eta_{i, j, \varepsilon}^{-1} \zeta_{i, j, \varepsilon} B_{2(\varepsilon i-\omega), j-1}^{-1} \zeta_{i, j, \varepsilon}^{-1}\right)^{\varepsilon}\right) u^{l}\left(\prod_{i=1}^{k}\left(B_{\varepsilon(2 i-1),-1} B_{2(\varepsilon i-\omega), 0}^{-1}\right)^{\varepsilon}\right) u^{-l} \\
= & \left(\prod_{i=1}^{k} \prod_{j=1}^{l}\left(\eta_{i, j, \varepsilon} B_{\varepsilon(2 i-1),-j} \eta_{i, j, \varepsilon}^{-1} \zeta_{i, j, \varepsilon} B_{2(\varepsilon i-\omega), j-1}^{-1} \zeta_{i, j, \varepsilon}^{-1}\right)^{\varepsilon}\right) \\
& \left(\prod_{i=1}^{k}\left(\eta_{i, j, \varepsilon} B_{\varepsilon(2 i-1),-l-1} \eta_{i, j, \varepsilon}^{-1} \zeta_{i, j, \varepsilon} B_{2(\varepsilon i-\omega), l}^{-1} \zeta_{i, j, \varepsilon}^{-1}\right)^{\varepsilon}\right) \\
= & \prod_{i=1}^{k} \prod_{j=1}^{l+1}\left(\eta_{i, j, \varepsilon} B_{\varepsilon(2 i-1),-j} \eta_{i, j, \varepsilon}^{-1} \zeta_{i, j, \varepsilon} B_{2(\varepsilon i-\omega), j-1}^{-1} \zeta_{i, j, \varepsilon}^{-1}\right)^{\varepsilon}
\end{aligned}
$$

Notemos que para cada $\varepsilon \in\{-1,1\}$ e para cada $l>0$, temos que

$$
O_{\varepsilon k,-l}=v^{2 \varepsilon k} u^{-l} v^{-2 \varepsilon k} u^{l}=v^{2 \varepsilon k} u^{-l} v^{-2 \varepsilon k} u^{l} v^{2 \varepsilon k} u^{-l} u^{l} v^{-2 \varepsilon k}=v^{2 \varepsilon k} u^{-l} O_{-\varepsilon k, l} u^{l} v^{-2 \varepsilon k} .
$$

Vamos usar a igualdade (4.14) e a fórmula (1)(que já mostramos ser verdadeira) para mostrar as fórmulas (2). Para $l>0$ e para $1 \leq i \leq k, 1 \leq j \leq l$ e $\varepsilon \in\{-1,1\}$, utilizando o Lema 4.3.5, nós podemos concluir que existem $\mu_{i, j, \varepsilon}, \nu_{i, j, \varepsilon}, \in \overline{\left\langle\sigma^{2}\right\rangle}$ tais que $v^{2 \varepsilon k} u^{-l} \eta_{i, j,-\varepsilon} B_{-\varepsilon(2 i-1),-j} \eta_{i, j,-\varepsilon}^{-1} u^{l} v^{-2 \varepsilon k}=$ $\mu_{i, j, \varepsilon} B_{\varepsilon(2 k-2 i+1), l-j} \mu_{i, j, \varepsilon}^{-1}$ e $v^{2 \varepsilon k} u^{-l} \zeta_{i, j,-\varepsilon} B_{2(-\varepsilon i+\omega), j-1} \zeta_{i, j,-\varepsilon}^{-1} u^{l} v^{-2 \varepsilon k}=\nu_{i, j, \varepsilon} B_{2(\varepsilon k-\varepsilon i+\omega),-l+j-1} \nu_{i, j, \varepsilon}^{-1}$. 
Assim, temos que

$$
\begin{aligned}
O_{\varepsilon k,-l} & =v^{2 \varepsilon k} u^{-l}\left(\prod_{i=1}^{k} \prod_{j=1}^{l}\left(\eta_{i, j,-\varepsilon} B_{-\varepsilon(2 i-1),-j} \eta_{i, j,-\varepsilon}^{-1} \zeta_{i, j,-\varepsilon} B_{2(-\varepsilon i+\omega), j-1}^{-1} \zeta_{i, j,-\varepsilon}^{-1}\right)^{-\varepsilon}\right) u^{l} v^{-2 \varepsilon k} \\
& =\prod_{i=1}^{k} \prod_{j=1}^{l}\left(\mu_{i, j, \varepsilon} B_{\varepsilon(2 k-2 i+1), l-j} \mu_{i, j, \varepsilon}^{-1} \nu_{i, j, \varepsilon} B_{2(\varepsilon k-\varepsilon i+\omega),-l+j-1}^{-1} \zeta_{i, j,-\varepsilon}^{-1}\right)^{-\varepsilon} \\
& =\prod_{i=1}^{k} \prod_{j=1}^{l}\left(\mu_{i, j, \varepsilon} B_{\varepsilon(2 i-1), j-1} \mu_{i, j, \varepsilon}^{-1} \nu_{i, j, \varepsilon} B_{2(\varepsilon i-\omega+1),-j}^{-1} \zeta_{i, j,-\varepsilon}^{-1}\right)^{-\varepsilon}
\end{aligned}
$$

\subsection{Classificação das classes de homotopia com a propriedade de Borsuk-Ulam}

Nesta seção, nós vamos classificar os elementos do conjunto $\left[\mathbb{T}^{2}, \mathbb{K}^{2}\right]$ que têm a propriedade de Borsuk-Ulam com respeito a involução $\tau_{1}$.

Para tanto, primeiramente vamos fixar uma notação de um fato da teoria de números.

Observação 4.4.1. Dado um número inteiro não nulo $t$, nós podemos escrever $t$ de modo único como

$$
t=(-1)^{j(t)} 2^{e(t)} o(t)
$$

sendo $j(t) \in\{0,1\}$, e $(t)$ um inteiro não negativo e o(t) um inteiro impar positivo.

Utilizando o resultado e a notação do Teorema 4.2.1, assim como a notação do diagrama (4.1), precisamente nós queremos mostrar a veracidade do seguinte resultado de classificação:

Teorema 4.4.2. Consideremos a involução livre de pontos fixos $\tau_{1}: \mathbb{T}^{2} \rightarrow \mathbb{T}^{2}$ definida por $\tau_{1}(x, y)=\left(x+\frac{1}{2}, y\right)$. Uma classe de homotopia $\beta \in\left[\mathbb{T}^{2}, \mathbb{K}^{2}\right]$ tem a propriedade de Borsuk-Ulam se, e somente se, uma das seguintes condiçôes é verdadeira:

1. $\Delta(\beta)$ é a classe de conjugação de um homomorfismo do tipo 3;

2. $\Delta(\beta)$ é a classe de conjugação de um homomorfismo do tipo 4 com $s_{1}$ impar, $r_{1}=0$ e $r_{2} \neq 0$;

3. $\Delta(\beta)$ é a classe de conjugação de um homomorfismo do tipo 4 com $s_{1}$ impar, $r_{1} \neq 0, r_{2} \neq 0$ $e e\left(r_{1}\right)>e\left(r_{2}\right)$.

Seja $\alpha \in\left[\mathbb{T}^{2}, * ; \mathbb{K}^{2}, *\right]$ uma classe de homotopia pontuada e seguindo a notação do diagrama (4.1) e da identificação (4.2), seja $h_{\alpha}=\Gamma(\alpha): \pi_{1}\left(\mathbb{T}^{2}\right) \rightarrow \pi_{1}\left(\mathbb{K}^{2}\right)$. Consideremos a família de homomorfismos listada no Teorema 4.2.1. Nas próximas sub-seções, nós vamos mostrar que as seguintes proposições são verdadeiras, o que é suficiente para que o Teorema 4.4.2 fique provado, de acordo com o Teorema 1.4.4, item 2.

Proposição 4.4.3. Se $h_{\alpha}$ é do tipo 1, então a não tem a propriedade de Borsuk-Ulam.

Proposição 4.4.4. Se $h_{\alpha}$ é do tipo 2, então a não tem a propriedade de Borsuk-Ulam.

Proposição 4.4.5. Se $h_{\alpha}$ é do tipo 3, então a tem a propriedade de Borsuk-Ulam.

Proposição 4.4.6. Se $h_{\alpha}$ é do tipo 4 com $s_{1}$ par, então $\alpha$ não tem a propriedade de Borsuk-Ulam.

Proposição 4.4.7. Se $h_{\alpha}$ é do tipo 4 com $s_{1}$ impar e $r_{1}=r_{2}=0$, então $\alpha$ não tem a propriedade de Borsuk-Ulam. 
Proposição 4.4.8. Se $h_{\alpha}$ é do tipo 4 com $s_{1}$ impar, $r_{1}=0$ e $r_{2} \neq 0$, então $\alpha$ tem a propriedade de Borsuk-Ulam.

Proposição 4.4.9. Se $h_{\alpha}$ é do tipo 4 com $s_{1}$ impar, $r_{1} \neq 0, r_{2} \neq 0$ e e $\left(r_{1}\right) \leq e\left(r_{2}\right)$, então $\alpha$ não tem a propriedade de Borsuk-Ulam.

Proposição 4.4.10. Se $h_{\alpha}$ é do tipo 4 com $s_{1}$ impar, $r_{1} \neq 0, r_{2} \neq 0$ e e $\left(r_{1}\right)>e\left(r_{2}\right)$, então $\alpha$ tem a propriedade de Borsuk-Ulam.

Nas demonstrações das proposições listadas, nós vamos usar um critério algébrico equivalente a uma classe de homotopia pontuada não ter a propriedade de Borsuk-Ulam. Mais precisamente, usaremos o seguinte resultado:

Proposição 4.4.11. Seja $\alpha \in\left[\mathbb{T}^{2}, * ; \mathbb{K}^{2}, *\right]$ uma classe de homotopia pontuada e seguindo a notação do diagrama (4.1), seja $h_{\alpha}=\Gamma(\alpha): \pi_{1}\left(\mathbb{T}^{2}\right) \rightarrow \pi_{1}\left(\mathbb{K}^{2}\right)$. Então $\alpha$ não tem a propriedade de BorsukUlam se, e somente se, existem tranças puras $a, b \in P_{2}\left(\mathbb{K}^{2}\right)$ tais que:
(i) $a l_{\sigma}(b)=b a$
(ii) $h_{\alpha}(1,0)=\left(p_{1}\right)_{\#}\left(a l_{\sigma}(a)\right)$
(iii) $h_{\alpha}(0,1)=\left(p_{1}\right)_{\#}(b)$

Demonstração. Lembremos que de acordo com a Observação 2.1.2, o grupo $P_{2}\left(\mathbb{T}^{2}\right)$ está identificado com o grupo $F(x, y) \oplus \mathbb{Z} \oplus \mathbb{Z}$. Ainda pela Observação 2.1.2, se $(w(x, y), m, n) \in P_{2}\left(\mathbb{T}^{2}\right)$, então $\left(p_{1}\right)_{\#}(w, m, n)=(m, n) \in \mathbb{Z} \oplus \mathbb{Z}$. Lembremos ainda, que existe um elemento $\sigma \in B_{2}\left(\mathbb{T}^{2}\right)$ tal que $\sigma^{2}=(B, 0,0)$, sendo $B=\left[x, y^{-1}\right] \in F(x, y)$.

Agora, de acordo com a Observação 4.3.1, $P_{2}\left(\mathbb{K}^{2}\right)$ está identificado com o grupo $F(u, v) \rtimes_{\theta}(\mathbb{Z} \rtimes \mathbb{Z})$. Se $(w(u, v) ; m, n) \in P_{2}\left(\mathbb{K}^{2}\right)$, então $\left(p_{1}\right)_{\#}(w ; m, n)=(m, n) \in \mathbb{Z} \rtimes \mathbb{Z}$. E também exite um elemento $\sigma \in B_{2}\left(\mathbb{K}^{2}\right)$ tal que $\sigma^{2}=(B ; 0,0)$, sendo $B=u v u v^{-1} \in F(u, v)$.

Observando as semelhanças entre as propriedades dos grupos de tranças do Toro e da garrafa de Klein, a demonstração da Proposição 4.4.11 nada mais é que uma reescrita da demonstração da Proposição 2.2.1 trocando $B_{2}\left(\mathbb{T}^{2}\right), P_{2}\left(\mathbb{T}^{2}\right)$ e $\pi_{1}\left(\mathbb{T}^{2}\right)$ por $B_{2}\left(\mathbb{K}^{2}\right), P_{2}\left(\mathbb{K}^{2}\right)$ e $\pi_{1}\left(\mathbb{K}^{2}\right)$, respectivamente, e utilizando a Observação 4.3.1 ao invés da Observação 2.1.2. Por este motivo, vamos omitir a prova.

Corolário 4.4.12. Consideremos duas classes de homotopia pontuadas $\alpha, \alpha^{\prime} \in\left[\mathbb{T}^{2}, * ; \mathbb{K}^{2}, *\right]$ e os homomorfismos $h_{\alpha}, h_{\alpha^{\prime}}: \pi_{1}\left(\mathbb{T}^{2}\right) \rightarrow \pi_{1}\left(\mathbb{K}^{2}\right)$. Suponhamos que

$$
h_{\alpha}:\left\{\begin{array}{l}
(1,0) \mapsto\left(r_{1}, s_{1}\right) \\
(0,1) \mapsto\left(r_{2}, s_{2}\right)
\end{array} \quad \text { e } \quad h_{\alpha^{\prime}}:\left\{\begin{array}{l}
(1,0) \mapsto\left(r_{1}, s_{1}^{\prime}\right) \\
(0,1) \mapsto\left(r_{2}, s_{2}^{\prime}\right)
\end{array}\right.\right.
$$

para algum $r_{1}, r_{2}, s_{1}, s_{1}^{\prime}, s_{2}, s_{2}^{\prime} \in \mathbb{Z}$. Se $s_{1} \equiv s_{1}^{\prime} \bmod 4$ e $s_{2} \equiv s_{2}^{\prime} \bmod 2$, então a tem a propriedade de Borsuk-Ulam se, e somente se, $\alpha^{\prime}$ tem a propriedade de Borsuk-Ulam.

Demonstração. Por contra positiva, para que o corolário fique demonstrado basta mostrar que $\alpha$ não tem a propriedade de Borsuk-Ulam se, e somente se, $\alpha^{\prime}$ não tem a propriedade de BorsukUlam. Devido a simetria do enunciado, bastra mostrar que uma direção é verdadeira. Suponhamos então que $\alpha$ não tem a propriedade de Borsuk-Ulam. Pela Proposição 4.4.11, existem tranças puras $a, b \in P_{2}\left(\mathbb{K}^{2}\right)$ que satisfazem
(i) $a l_{\sigma}(b)=b a$
(ii) $h_{\alpha}(1,0)=\left(p_{1}\right)_{\#}\left(a l_{\sigma}(a)\right)$
(iii) $h_{\alpha}(0,1)=\left(p_{1}\right)_{\#}(b)$.

Por hipótese, existem $k_{1}, k_{2} \in \mathbb{Z}$ tais que $s_{1}^{\prime}=s_{1}+4 k_{1}$ e $s_{2}^{\prime}=s_{2}+2 k_{2}$. Tomamos os seguintes elementos de $P_{2}\left(\mathbb{K}^{2}\right)$ :

$$
a^{\prime}=a\left(\mathbb{1} ; 0,2 k_{1}\right) \quad \text { e } \quad b^{\prime}=b\left(\mathbb{1} ; 0,2 k_{2}\right) .
$$


Usando a Observação 4.3.1, é fácil ver que $\left(\mathbb{1} ; 0,2 k_{1}\right)$ e $\left(\mathbb{1} ; 0,2 k_{2}\right)$ pertencem ao centro de $P_{2}\left(\mathbb{K}^{2}\right)$ e comutam com o elemento $\sigma \in B_{2}\left(\mathbb{K}^{2}\right)$. Assim temos que

$$
\begin{aligned}
& a^{\prime} l_{\sigma}\left(b^{\prime}\right)=a\left(\mathbb{1} ; 0,2 k_{1}\right) l_{\sigma}\left(b\left(\mathbb{1} ; 0,2 k_{2}\right)\right)=a l_{\sigma}(b)\left(\mathbb{1} ; 0,2 k_{1}+2 k_{2}\right) \\
& \stackrel{(i)}{=} b a\left(\mathbb{1} ; 0,2 k_{1}+2 k_{2}\right)=b\left(\mathbb{1} ; 0,2 k_{2}\right) a\left(\mathbb{1} ; 0,2 k_{1}\right)=b^{\prime} a^{\prime}, \\
&\left(p_{1}\right)_{\#}\left(a^{\prime} l_{\sigma}\left(a^{\prime}\right)\right) \stackrel{(i i)}{=}\left(p_{1}\right)_{\#}\left(a\left(\mathbb{1} ; 0,2 k_{1}\right) l_{\sigma}\left(a\left(\mathbb{1} ; 0,2 k_{1}\right)\right)\right)=\left(p_{1}\right)_{\#}\left(a l_{\sigma}(a)\left(\mathbb{1} ; 0,4 k_{1}\right)\right)\left(r_{1}, s_{1}\right)\left(0,4 k_{1}\right)=\left(r_{1}, s_{1}^{\prime}\right)=h_{\alpha^{\prime}}(1,0), \\
&\left(p_{1}\right)_{\#}\left(b^{\prime}\right)=\left(p_{1}\right)_{\#}\left(b\left(\mathbb{1} ; 0,2 k_{2}\right)\right) \stackrel{(i i i)}{=}\left(r_{2}, s_{2}\right)\left(0,2 k_{2}\right)=\left(r_{2}, s_{2}^{\prime}\right)=h_{\alpha^{\prime}}(0,1)
\end{aligned}
$$

e portanto, pela Proposição 4.4.11, segue que $\alpha^{\prime}$ não tem a propriedade de Borsuk-Ulam.

\subsubsection{Prova das Proposições 4.4.3, 4.4.4, 4.4.6, 4.4.7 e 4.4.9}

Nesta subseção nós vamos provar as Proposições 4.4.3, 4.4.4, 4.4.6, 4.4.7 e 4.4.9.

Primeiramente, vamos mostrar alguns resultados preliminares.

Lema 4.4.13. Seja $(r, s) \in \mathbb{Z} \rtimes \mathbb{Z}=\pi_{1}\left(\mathbb{K}^{2}\right)$. Suponhamos que $\alpha \in\left[\mathbb{T}^{2}, * ; \mathbb{K}^{2}, *\right]$ é uma classe de homotopia tal que o homomorfismo $h_{\alpha}=\Gamma(\alpha): \pi_{1}\left(\mathbb{T}^{2}\right) \rightarrow \pi_{1}\left(\mathbb{K}^{2}\right)$ tem os seguintes valores nos geradores:

$$
h_{\alpha}(1,0)=(r, s) \quad \text { e } \quad h_{\alpha}(0,1)=(0,0) .
$$

Então a não tem a propriedade de Borsuk-Ulam.

Demonstração. Consideremos os seguintes elementos de $P_{2}\left(\mathbb{K}^{2}\right)$ :

$$
a=\left(u^{r} v^{s} ; 0,0\right) \text { e } \quad b=(\mathbb{1} ; 0,0) .
$$

Vamos mostrar que $a, b$ e $h_{\alpha}$ satisfazem $(i)$, (ii) e (iii) da Proposição 4.4.11, donde seguirá que $\alpha$ não tem a propriedade de Borsuk-Ulam. Usaremos a Observação 4.3.1 e a comutatividade do diagrama (4.5).

$$
\begin{aligned}
& a l_{\sigma}(b)=\left(u^{r} v^{s} ; 0,0\right) l_{\sigma}(\mathbb{1} ; 0,0)=\left(u^{r} v^{s} ; 0,0\right)(\mathbb{1} ; 0,0)=(\mathbb{1} ; 0,0)\left(u^{r} v^{s} ; 0,0\right)=b a ; \\
& \left(p_{1}\right)_{\#}\left(a l_{\sigma}(a)\right)=\left(p_{1}\right)_{\#}\left(\left(u^{r} v^{s} ; 0,0\right) l_{\sigma}\left(u^{r} v^{s} ; 0,0\right)\right)=(0,0)(r, s)=h_{\alpha}(1,0) ; \\
& \left(p_{1}\right)_{\#}(b)=\left(p_{1}\right)_{\#}(\mathbb{1} ; 0,0)=(0,0)=h_{\alpha}(0,1) .
\end{aligned}
$$

Lema 4.4.14. Consideremos uma classe de homotopia $\alpha \in\left[\mathbb{T}^{2}, * ; \mathbb{T}^{2}, *\right]$ e seguindo a notação da Seção 1.2, seja o homomorfismo $h_{\alpha}=\Gamma_{\mathbb{T}^{2}, \mathbb{T}^{2}}(\alpha): \pi_{1}\left(\mathbb{T}^{2}\right) \rightarrow \pi_{1}\left(\mathbb{T}^{2}\right)$. Suponhamos que

$$
h_{\alpha}(1,0)=\left(2 m_{1}+1,2 n_{1}\right) \quad \text { e } \quad h_{\alpha}(1,0)=\left(m_{2}, n_{2}\right)
$$

para algum $m_{1}, n_{1}, m_{2}, n_{2} \in \mathbb{Z}$. Então $\alpha$ tem um representante $f:\left(\mathbb{T}^{2}, *\right) \rightarrow\left(\mathbb{T}^{2}, *\right)$ que é $\left(\tau_{1}, \tau_{1}\right)$ equivariante, isto é, $\alpha=[f]$ e $f\left(\tau_{1}(z)\right)=\tau_{1}(f(z))$ para todo $z \in \mathbb{T}^{2}$.

Demonstração. Seja $\psi: \pi_{1}\left(\mathbb{T}^{2}\right) \rightarrow \pi_{1}\left(\mathbb{T}^{2}\right)$ definido nos geradores por

$$
\psi(1,0)=\left(2 m_{1}+1, n_{1}\right) \quad \text { e } \quad \psi(0,1)=\left(2 m_{2}, n_{2}\right)
$$

e estendido por linearidade. Lembremos que na Seção 2.1, nós mostramos que os homomorfismos $\left(p_{\tau_{1}}\right)_{\#}: \pi_{1}\left(\mathbb{T}^{2}\right) \rightarrow \pi_{1}\left(\mathbb{T}^{2}\right)$ e $\theta_{\tau_{1}}: \pi_{1}\left(\mathbb{T}^{2}\right) \rightarrow \mathbb{Z}_{2}$ têm os seguintes valores nos geradores:

$$
\left(p_{\tau_{1}}\right)_{\#}(1,0)=(2,0) \quad \text { e } \quad\left(p_{\tau_{1}}\right)_{\#}(0,1)=(0,1) ;
$$




$$
\theta_{\tau_{1}}(1,0)=\overline{1} \quad \text { e } \quad \theta_{\tau_{1}}(0,1)=\overline{0}
$$

Vamos mostrar que o seguinte diagrama é comutativo, donde seguirá a tese do enunciado, de acordo com o Lema 1.4.1:

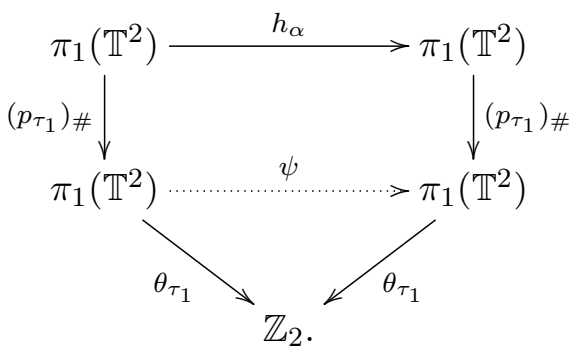

$$
\begin{aligned}
& \left(\left(p_{\tau_{1}}\right)_{\#} \circ h_{\alpha}\right)(1,0)=\left(p_{\tau_{1}}\right)_{\#}\left(2 m_{1}+1,2 n_{1}\right)=\left(2 m_{1}+1\right)\left(p_{\tau_{1}}\right)_{\#}(1,0)+2 n_{1}\left(p_{\tau_{1}}\right)_{\#}(0,1) \\
& =\left(2 m_{1}+1\right)(2,0)+2 n_{1}(0,1)=2\left(2 m_{1}+1, n_{1}\right)=2 \psi(1,0)=\left(\psi \circ\left(p_{\tau_{1}}\right)_{\#}\right)(1,0) \\
& \left(\left(p_{\tau_{1}}\right)_{\#} \circ h_{\alpha}\right)(0,1)=\left(p_{\tau_{1}}\right)_{\#}\left(m_{2}, n_{2}\right)=m_{2}\left(p_{\tau_{1}}\right)_{\#}(1,0)+n_{2}\left(p_{\tau_{1}}\right)_{\#}(0,1) \\
& =m_{2}(2,0)+n_{2}(0,1)=\left(2 m_{2}, n_{2}\right)=\psi(0,1)=\left(\psi \circ\left(p_{\tau_{1}}\right)_{\#}\right)(0,1) \\
& \left(\theta_{\tau_{1}} \circ \psi\right)(1,0)=\theta_{\tau_{1}}\left(2 m_{1}+1, n_{1}\right)=\left(2 m_{1}+1\right) \theta_{\tau_{1}}(1,0)+n_{1} \theta_{\tau_{1}}(0,1)=\left(2 m_{1}+1\right) \overline{1}+n_{1} \overline{0}=\overline{1}=\theta_{\tau_{1}}(1,0) \\
& \left(\theta_{\tau_{1}} \circ \psi\right)(0,1)=\theta_{\tau_{1}}\left(2 m_{2}, n_{2}\right)=2 m_{2} \theta_{\tau_{1}}(1,0)+n_{2} \theta_{\tau_{1}}(0,1)=2 m_{2} \overline{1}+n_{2} \overline{0}=\overline{0}=\theta_{\tau_{1}}(0,1) .
\end{aligned}
$$

Lema 4.4.15. Sejam $f:\left(\mathbb{T}^{2}, *\right) \rightarrow\left(\mathbb{T}^{2}, *\right)$ e $g:\left(\mathbb{T}^{2}, *\right) \rightarrow\left(\mathbb{K}^{2}, *\right)$ funçôes tais que para todo $z \in \mathbb{T}^{2}$ valem as seguintes condiçôes:
(1) $f\left(\tau_{1}(z)\right)=\tau_{1}(f(z))$
(2) $g\left(\tau_{1}(z)\right) \neq g(z)$,

sendo $\tau_{1}: \mathbb{T}^{2} \rightarrow \mathbb{T}^{2}$ a involução livre de pontos fixas como definida no início deste capítulo. Então a classe de homotopia $[g \circ f] \in\left[\mathbb{T}^{2}, * ; \mathbb{K}^{2}, *\right]$ não tem a propriedade de Borsuk-Ulam.

Demonstração. Para todo $z \in \mathbb{T}^{2}$ temos que

$$
(g \circ f)\left(\tau_{1}(z)\right)=g\left(f\left(\tau_{1}(z)\right)\right) \stackrel{(1)}{=} g\left(\tau_{1}(f(z))\right) \stackrel{(2)}{\neq} g(f(z))=(g \circ f)(z)
$$

e portanto, a classe de homotopia $[g \circ f]$ não tem a propriedade de Borsuk-Ulam.

Demonstração da Proposição 4.4.3. Queremos mostrar que se $\alpha \in\left[\mathbb{T}^{2}, * ; \mathbb{K}^{2} *\right]$ é uma classe de homotopia cujo homomorfismo $h_{\alpha}: \pi_{1}\left(\mathbb{T}^{2}\right) \rightarrow \pi_{1}\left(\mathbb{K}^{2}\right)$ é tal que

$$
h_{\alpha}(1,0)=\left(i, 2 s_{1}+1\right) \quad \text { e } h_{\alpha}(0,1)=\left(0,2 s_{2}\right),
$$

para algum $i \in\{0,1\}$ e para algum $s_{1}, s_{2} \in \mathbb{Z}$, então $\alpha$ não tem a propriedade de Borsuk-Ulam. Pelo Corolário 4.4.12, para que esta Proposição fique provada, basta mostrarmos que se $\alpha \in\left[\mathbb{T}^{2}, * ; \mathbb{K}^{2} *\right]$ é uma classe de homotopia cujo homomorfismo $h_{\alpha}: \pi_{1}\left(\mathbb{T}^{2}\right) \rightarrow \pi_{1}\left(\mathbb{K}^{2}\right)$ é tal que

$$
h_{\alpha}(1,0)=(i, 2 j+1) \quad \text { e } \quad h_{\alpha}(0,1)=(0,0),
$$


para algum $i, j \in\{0,1\}$, então $\alpha$ não tem a propriedade de Borsuk-Ulam. Mas este fato segue aplicando o Lema 4.4.13 para o elemento $(i, 2 j+1) \in \mathbb{Z} \rtimes \mathbb{Z}$.

Demonstração da Proposição 4.4.4. Queremos mostrar que se $\alpha \in\left[\mathbb{T}^{2}, * ; \mathbb{K}^{2} *\right]$ é uma classe de homotopia cujo homomorfismo $h_{\alpha}: \pi_{1}\left(\mathbb{T}^{2}\right) \rightarrow \pi_{1}\left(\mathbb{K}^{2}\right)$ é tal que

$$
h_{\alpha}(1,0)=\left(i, 2 s_{1}+1\right) \quad \text { e } \quad h_{\alpha}(0,1)=\left(i, 2 s_{2}+1\right),
$$

para algum $i \in\{0,1\}$ e para algum $s_{1}, s_{2} \in \mathbb{Z}$, então $\alpha$ não tem a propriedade de Borsuk-Ulam. Pelo Corolário 4.4.12, para que esta Proposição fique provada, basta mostrarmos que se $\alpha \in\left[\mathbb{T}^{2}, * ; \mathbb{K}^{2} *\right]$ é uma classe de homotopia cujo o homomorfismo $h_{\alpha}: \pi_{1}\left(\mathbb{T}^{2}\right) \rightarrow \pi_{1}\left(\mathbb{K}^{2}\right)$ é tal que

$$
h_{\alpha}(1,0)=(i, 2 j+1) \text { e } h_{\alpha}(0,1)=(i, 1),
$$

para algum $i, j \in\{0,1\}$, então $\alpha$ não tem a propriedade de Borsuk-Ulam.

Pelo Lema 4.4.13, se $\alpha_{0} \in\left[\mathbb{T}^{2}, * ; \mathbb{K}^{2}, *\right]$ é uma classe de homotopia cujo homomorfismo $h_{\alpha_{0}}: \pi_{1}\left(\mathbb{T}^{2}\right) \rightarrow \pi_{1}\left(\mathbb{K}^{2}\right)$ é tal que

$$
h_{\alpha_{0}}(1,0)=(i, 1) \quad \text { e } \quad h_{\alpha_{0}}(0,1)=(0,0),
$$

então $\alpha_{0}$ tem um representante $g:\left(\mathbb{T}^{2}, *\right) \rightarrow\left(\mathbb{K}^{2}, *\right)$ tal que $g\left(\tau_{1}(z)\right) \neq g(z)$ para todo $z \in \mathbb{T}^{2}$. Lembremos que $h_{\alpha_{0}}=g_{\#}$.

Pelo Lema 4.4.14, se $\alpha_{1} \in\left[\mathbb{T}^{2}, * ; \mathbb{T}^{2}, *\right]$ é uma classe de homotopia cujo homomorfismo $h_{\alpha_{1}}=\Gamma_{\mathbb{T}^{2}, \mathbb{T}^{2}}\left(\alpha_{1}\right): \pi_{1}\left(\mathbb{T}^{2}\right) \rightarrow \pi_{1}\left(\mathbb{T}^{2}\right)$ é tal que

$$
h_{\alpha_{1}}(1,0)=(2 j+1,0) \text { e } h_{\alpha_{1}}(0,1)=(1,0),
$$

então $\alpha_{1}$ tem um representante $f:\left(\mathbb{T}^{2}, *\right) \rightarrow\left(\mathbb{T}^{2}, *\right)$ que é $\left(\tau_{1}, \tau_{1}\right)$-equivariante. Lembremos que $h_{\alpha_{1}}=f_{\#}$.

Vamos mostrar que $\alpha=[g \circ f]$, donde seguirá pelo Lema 4.4 .15 que $\alpha$ não tem a propriedade de Borsuk-Ulam. Pelo Teorema 1.2.1, basta mostrarmos que $h_{\alpha}=(g \circ f)_{\#}$. Temos:

$$
(g \circ f)_{\#}(1,0)=\left(h_{\alpha_{0}}\left(h_{\alpha_{1}}(1,0)\right)\right)=h_{\alpha_{0}}(2 j+1,0)=h_{\alpha_{0}}(1,0)^{2 j+1}=(i, 1)^{2 j+1}=(i, 2 j+1)=h_{\alpha}(1,0),
$$

e

$(g \circ f)_{\#}(0,1)=\left(h_{\alpha_{0}}\left(h_{\alpha_{1}}(0,1)\right)\right)=h_{\alpha_{0}}(1,0)=(i, 1)=h_{\alpha}(0,1)$.

Demonstração da Proposição 4.4.6. Queremos mostrar que se $\alpha \in\left[\mathbb{T}^{2}, * ; \mathbb{K}^{2} *\right]$ é uma classe de homotopia cujo homomorfismo $h_{\alpha}: \pi_{1}\left(\mathbb{T}^{2}\right) \rightarrow \pi_{1}\left(\mathbb{K}^{2}\right)$ é tal que

$$
h_{\alpha}(1,0)=\left(r_{1}, 2 s_{1}\right) \quad \text { e } \quad h_{\alpha}(0,1)=\left(r_{2}, 2 s_{2}\right),
$$

para algum $r_{1}, r_{2}, s_{1}, s_{2} \in \mathbb{Z}$, com $r_{1} \geq 0$ e $s_{1}$ par, então $\alpha$ não tem a propriedade de BorsukUlam. Como $2 s_{1} \equiv 0 \bmod 4$ e $2 s_{2} \equiv 0 \bmod 2$, pelo Corolário 4.4.12, para que esta Proposição fique provada, basta mostrarmos que se $\alpha \in\left[\mathbb{T}^{2}, * ; \mathbb{K}^{2} *\right]$ é uma classe de homotopia cujo homomorfismo $h_{\alpha}: \pi_{1}\left(\mathbb{T}^{2}\right) \rightarrow \pi_{1}\left(\mathbb{K}^{2}\right)$ é tal que

$$
h_{\alpha}(1,0)=\left(r_{1}, 0\right) \quad \text { e } \quad h_{\alpha}(0,1)=\left(r_{2}, 0\right)
$$

para algum $r_{1}, r_{2} \in \mathbb{Z}$, com $r_{1} \geq 0$, então $\alpha$ não tem a propriedade de Borsuk-Ulam. Vamos mostrar que existem tranças puras $a, b \in P_{2}\left(\mathbb{K}^{2}\right)$ que juntamente com o homomorfismo $h_{\alpha}$, satisfazem os 
itens $(i)$, (ii) e (iii) da Proposição 4.4.11, donde seguirá o resultado. Utilizaremos fortemente a Observação 4.3 .1 e a comutatividade do diagrama (4.5).

1. Suponhamos $r_{1}$ par. Seja $r \in \mathbb{Z}$ tal que $r_{1}=2 r$. Consideremos os seguintes elementos de $P_{2}\left(\mathbb{K}^{2}\right)$ :

$$
a=(\mathbb{1} ; r, 0) \quad \text { e } \quad b=\left(\mathbb{1} ; r_{2}, 0\right) .
$$

Temos que

$$
a l_{\sigma}(b)=(\mathbb{1} ; r, 0) l_{\sigma}\left(\mathbb{1} ; r_{2}, 0\right)=(\mathbb{1} ; r, 0)\left(\mathbb{1} ; r_{2}, 0\right)=\left(\mathbb{1} ; r+r_{2}, 0\right)=\left(\mathbb{1} ; r_{2}, 0\right)(\mathbb{1} ; r, 0)=b a
$$

e portanto, vale $(i)$. Temos também que

$\left(p_{1}\right)_{\#}\left(a l_{\sigma}(a)\right)=\left(p_{1}\right)_{\#}\left((\mathbb{1} ; r, 0) l_{\sigma}(\mathbb{1} ; r, 0)\right)=(r, 0)(r, 0)=\left(r_{1}, 0\right)=h_{\alpha}(1,0) \mathrm{e}$

$\left(p_{1}\right)_{\#}(b)=\left(p_{1}\right)_{\#}\left(\mathbb{1} ; r_{2}, 0\right)=\left(r_{2}, 0\right)=h_{\alpha}(0,1)$

e portanto, valem (ii) e (iii).

2. Suponhamos $r_{1}$ impar. Seja $r \in \mathbb{Z}$ tal que $r_{1}=2 r+1$. Consideremos os seguintes elementos de $P_{2}\left(\mathbb{K}^{2}\right)$ :

$$
a=(u ; r, 0) \quad \text { e } \quad b=\left(B^{-r_{2}} ; r_{2}, 0\right) .
$$

Temos que

$$
\begin{aligned}
a l_{\sigma}(b) & =(u ; r, 0) l_{\sigma}\left(B^{-r_{2}} ; r_{2}, 0\right)=(u ; r, 0) l_{\sigma}\left(B^{-r_{2}} ; 0,0\right) l_{\sigma}\left(\mathbb{1} ; r_{2}, 0\right) \\
& =(u ; r, 0)\left(B^{-r_{2}} ; 0,0\right)\left(\mathbb{1} ; r_{2}, 0\right)=\left(u \theta(r, 0)(B)^{-r_{2}} ; r+r_{2}, 0\right) \\
& =\left(u B^{-r_{2}} ; r+r_{2}, 0\right)=\left(B^{-r_{2}} B^{r_{2}} u B^{-r_{2}} ; r_{2}+r ; 0\right) \\
& =\left(B^{-r_{2}} \theta\left(r_{2}, 0\right)(u) ; r_{2}+r ; 0\right)=\left(B^{-r_{2}} ; r_{2}, 0\right)(u ; r, 0)=b a
\end{aligned}
$$

e portanto, vale $(i)$. Temos também que

$\left(p_{1}\right)_{\#}\left(a l_{\sigma}(a)\right)=\left(p_{1}\right)_{\#}\left((u ; r, 0) l_{\sigma}(u ; r, 0)\right)=(r, 0) g(u)(r, 0)=(r, 0)(1,0)(r, 0)=\left(r_{1}, 0\right)=h_{\alpha}(1,0) \mathrm{e}$

$\left(p_{1}\right)_{\#}\left(B^{-r_{2}} ; r_{2}, 0\right)=\left(r_{2}, 0\right)=h_{\alpha}(0,1)$

e portanto, valem $(i i)$ e (iii).

Demonstração da Proposição 4.4.7. Queremos mostrar que se $\alpha \in\left[\mathbb{T}^{2}, * ; \mathbb{K}^{2} *\right]$ é uma classe de homotopia cujo homomorfismo $h_{\alpha}: \pi_{1}\left(\mathbb{T}^{2}\right) \rightarrow \pi_{1}\left(\mathbb{K}^{2}\right)$ é tal que

$$
h_{\alpha}(1,0)=\left(0,2 s_{1}\right) \quad \text { e } \quad h_{\alpha}(0,1)=\left(0,2 s_{2}\right),
$$

para algum $s_{1}, s_{2} \in \mathbb{Z}$, com $s_{1}$ ímpar, então $\alpha$ não tem a propriedade de Borsuk-Ulam. Como $2 s_{1} \equiv 2 \bmod 4$ e $2 s_{2} \equiv 0 \bmod 2$, pelo Corolário 4.4.12, para que este resultado fique provado, basta mostrarmos que se $\alpha \in\left[\mathbb{T}^{2}, * ; \mathbb{K}^{2} *\right]$ é uma classe de homotopia cujo homomorfismo $h_{\alpha}: \pi_{1}\left(\mathbb{T}^{2}\right) \rightarrow \pi_{1}\left(\mathbb{K}^{2}\right)$ é tal que

$$
h_{\alpha}(1,0)=(0,2) \text { e } h_{\alpha}(0,1)=(0,0),
$$

então $\alpha$ não tem a propriedade de Borsuk-Ulam. Mas este fato segue aplicando o Lema 4.4.13 para o elemento $(0,2) \in \mathbb{Z} \rtimes \mathbb{Z}$.

Demonstração da Proposição 4.4.9. Queremos mostrar que se $\alpha \in\left[\mathbb{T}^{2}, * ; \mathbb{K}^{2} *\right]$ é uma classe de 
homotopia cujo homomorfismo $h_{\alpha}: \pi_{1}\left(\mathbb{T}^{2}\right) \rightarrow \pi_{1}\left(\mathbb{K}^{2}\right)$ é tal que

$$
h_{\alpha}(1,0)=\left(r_{1}, 2 s_{1}\right) \quad \text { e } \quad h_{\alpha}(0,1)=\left(r_{2}, 2 s_{2}\right),
$$

para algum $r_{1}, s_{1}, r_{2}, s_{2} \in \mathbb{Z}$, com $r_{1} \geq 0, s_{1}$ ímpar e $e\left(r_{1}\right) \leq e\left(r_{2}\right)$, então $\alpha$ não tem a propriedade de Borsuk-Ulam. Notemos que como $s_{1}$ e $o\left(r_{1}\right)$ são ímpares, então $2 s_{1} \equiv 2 o\left(r_{1}\right) \bmod 4$. Notemos também que $m=(-1)^{j\left(r_{2}\right)} 2^{e\left(r_{2}\right)-e\left(r_{1}\right)} o\left(r_{2}\right)$ é um número inteiro. Como $2 m \equiv 2 s_{2} \bmod 2$, então pelo Corolário 4.4.12, para que o resultado fique provado, basta mostrarmos que se $\alpha \in\left[\mathbb{T}^{2}, * ; \mathbb{K}^{2} *\right]$ é uma classe de homotopia cujo homomorfismo $h_{\alpha}: \pi_{1}\left(\mathbb{T}^{2}\right) \rightarrow \pi_{1}\left(\mathbb{K}^{2}\right)$ é tal que

$$
h_{\alpha}(1,0)=\left(r_{1}, 2 o\left(r_{1}\right)\right) \text { e } h_{\alpha}(0,1)=\left(r_{2}, 2 m\right),
$$

então $\alpha$ não tem a propriedade de Borsuk-Ulam.

Pelo Lema 4.4.13, se $\alpha_{0} \in\left[\mathbb{T}^{2}, * ; \mathbb{K}^{2}, *\right]$ é uma classe de homotopia cujo homomorfismo $h_{\alpha_{0}}: \pi_{1}\left(\mathbb{T}^{2}\right) \rightarrow \pi_{1}\left(\mathbb{K}^{2}\right)$ é tal que

$$
h_{\alpha_{0}}(1,0)=\left(2^{e\left(r_{1}\right)}, 2\right) \quad \text { e } \quad h_{\alpha_{0}}(0,1)=(0,0),
$$

então $\alpha_{0}$ tem um representante $g:\left(\mathbb{T}^{2}, *\right) \rightarrow\left(\mathbb{K}^{2}, *\right)$ tal que $g\left(\tau_{1}(z)\right) \neq g(z)$ para todo $z \in \mathbb{T}^{2}$. Lembremos que $h_{\alpha_{0}}=g_{\#}$.

Pelo Lema 4.4.14, se $\alpha_{1} \in\left[\mathbb{T}^{2}, * ; \mathbb{T}^{2}, *\right]$ é uma classe de homotopia cujo homomorfismo $h_{\alpha_{1}}=\Gamma_{\mathbb{T}^{2}, \mathbb{T}^{2}}\left(\alpha_{1}\right): \pi_{1}\left(\mathbb{T}^{2}\right) \rightarrow \pi_{1}\left(\mathbb{T}^{2}\right)$ é tal que

$$
h_{\alpha_{1}}(1,0)=\left(o\left(r_{1}\right), 0\right) \quad \text { e } \quad h_{\alpha_{1}}(0,1)=(m, 0),
$$

então $\alpha_{1}$ tem um representante $f:\left(\mathbb{T}^{2}, *\right) \rightarrow\left(\mathbb{T}^{2}, *\right)$ que é $\left(\tau_{1}, \tau_{1}\right)$-equivariante. Lembremos que $h_{\alpha_{1}}=f_{\#}$.

Vamos mostrar que $\alpha=[g \circ f]$, donde seguirá pelo Lema 4.4 .15 que $\alpha$ não tem a propriedade de Borsuk-Ulam. Pelo Teorema 1.2.1, basta mostrarmos que $h_{\alpha}=(g \circ f)_{\#}$.

$$
\begin{aligned}
(g \circ f)_{\#}(1,0) & =\left(h_{\alpha_{0}}\left(h_{\alpha_{1}}(1,0)\right)\right)=h_{\alpha_{0}}\left(o\left(r_{1}\right), 0\right)=h_{\alpha_{0}}(1,0)^{o\left(r_{1}\right)} \\
& =\left(2^{e\left(r_{1}\right)}, 2\right)^{o\left(r_{1}\right)}=\left(2^{e\left(r_{1}\right)} o\left(r_{1}\right), 2 o\left(r_{1}\right)\right)=\left(r_{1}, 2 o\left(r_{1}\right)\right)=h_{\alpha}(1,0) \\
(g \circ f)_{\#}(0,1) & =\left(h_{\alpha_{0}}\left(h_{\alpha_{1}}(0,1)\right)\right)=h_{\alpha_{0}}(m, 0)=h_{\alpha_{0}}(1,0)^{m}=\left(2^{e\left(r_{1}\right)}, 2\right)^{m} \\
& =\left(2^{e\left(r_{1}\right)} m, 2 m\right)=\left(2^{e\left(r_{1}\right)}(-1)^{j\left(r_{2}\right)} 2^{e\left(r_{2}\right)-e\left(r_{1}\right)} o\left(r_{2}\right), 2 m\right) \\
& =\left(r_{2}, 2 m\right)=h_{\alpha}(0,1) .
\end{aligned}
$$

\subsubsection{Prova da Proposição 4.4.5}

O objetivo desta sub-seção é demonstrar a Proposição 4.4.5. Vamos primeiramente enunciar alguns resultados preliminares. Usaremos fortemente as notações e os resultados da Seção 4.3 .

Lema 4.4.16. Seja $\alpha \in\left[\mathbb{T}^{2}, * ; \mathbb{K}^{2}, *\right]$ uma classe de homotopia cujo homomorfismo $h_{\alpha}=\Gamma(\alpha): \pi_{1}\left(\mathbb{T}^{2}\right) \rightarrow \pi_{1}\left(\mathbb{K}^{2}\right)$ é tal que

$$
h_{\alpha}(1,0)=(0,2 i) \quad e \quad h_{\alpha}(0,1)=(j, 1)
$$


para algum $i, j \in\{0,1\}$. Utilizando a notação das Proposições 4.3.6 e 4.3.7, temos que se a não tem a propriedade de Borsuk-Ulam, então existem elementos $x, y \in{\overline{\left\langle\sigma^{2}\right\rangle}}_{\mathrm{ab}}$ e $(m, n) \in \mathbb{Z} \rtimes \mathbb{Z} q u e$ satisfazem uma das seguintes equações em ${\overline{\left\langle\sigma^{2}\right\rangle}}_{\mathrm{ab}}$ :

$$
\begin{aligned}
\mu_{1}(x)+\nu_{1}(y)= & \widetilde{I}_{2 n-i}-\widetilde{T}_{-2 m}+\widetilde{D}_{2 n-i,-2 j}-\widetilde{O}_{2 n-i,-2 m} \\
& -(j+m+1) B_{0,0}-m B_{0,-2 m}+B_{4 n-2 i, 0}+(j-1) B_{4 n-2 i,-2 m} \\
\mu_{2}(x)+\nu_{2}(y)= & \widetilde{I}_{2 n-i+1}-\widetilde{W}_{-2 m+2 j}+\widetilde{D}_{2 n-i+1,-2 j} \\
& +(j-m) B_{0,0}+(-m+1) B_{0,-2 m+2 j}+j B_{4 n-2 i+2,0},
\end{aligned}
$$

sendo

$$
\begin{aligned}
\mu_{1}(x) & =\left(c_{0,-2 m}\right)_{\mathrm{ab}}(x)-\theta(j, 1)_{\mathrm{ab}}(x) \\
\nu_{1}(y) & =\left(\theta(-m, 0)_{\mathrm{ab}} \circ \rho_{\mathrm{ab}}\right)(y)-\left(c_{4 n-2 i,-2 m}\right)_{\mathrm{ab}}(y) \\
\mu_{2}(x) & =\left(c_{0,-2 m+2 j}\right)_{\mathrm{ab}}(x)-\theta(j, 1)_{\mathrm{ab}}(x) \\
\nu_{2}(y) & =\left(\theta(-m+2 j, 1)_{\mathrm{ab}} \circ \rho_{\mathrm{ab}}\right)(y)-\left(c_{4 n-2 i+2,0}\right)_{\mathrm{ab}}(y) .
\end{aligned}
$$

Demonstração. Pela Proposição 4.4.11, como $\alpha$ não tem a propriedade de Borsuk-Ulam, existem elementos de $P_{2}\left(\mathbb{K}^{2}\right)$

$$
a=\left(w ; m_{1}, n_{1}\right) \quad \text { e } \quad b=\left(z ; m_{2}, n_{2}\right)
$$

tais que
(i) $a l_{\sigma}(b)=b a$
(ii) $\left(p_{1}\right)_{\#}\left(a l_{\sigma}(a)\right)=(0,2 i)$
(iii) $\left(p_{1}\right)_{\#}(b)=(j, 1)$.

Pela Observação 4.3 .1 e por (iii), temos que $\left(m_{2}, n_{2}\right)=(j, 1)$. Pelo Proposição 4.3.4, existem $x, y \in \overline{\left\langle\sigma^{2}\right\rangle}$ tais que $w=u^{a_{1}} v^{a_{2}} x$ e $z=u^{b_{1}} v^{b_{2}} y$, sendo $g(w)=\left(a_{1}, a_{2}\right)$ e $g(z)=\left(b_{1}, b_{2}\right)$. Logo,

$$
a=\left(u^{a_{1}} v^{a_{2}} x ; m_{1}, n_{1}\right) \quad \text { e } \quad b=\left(u^{b_{1}} v^{b_{2}} y ; j, 1\right) .
$$

Usando as Observações 4.3.1 e 4.3.3, a comutatividade do diagrama (4.5) e o item (ii), temos que

$$
\begin{aligned}
(0,2 i) & =\left(p_{1}\right)_{\#}\left(a l_{\sigma}(a)\right)=\left(p_{1}\right)_{\#}\left(u^{a_{1}} v^{a_{2}} x ; m_{1}, n_{1}\right)\left(p_{1}\right)_{\#}\left(l_{\sigma}\left(u^{a_{1}} v^{a_{2}} x ; m_{1}, n_{1}\right)\right) \\
& =\left(m_{1}, n_{1}\right)\left(p_{1}\right)_{\#}\left(l_{\sigma}\left(u^{a_{1}} v^{a_{2}} x ; 0,0\right)\right)\left(p_{1}\right)_{\#}\left(l_{\sigma}\left(\mathbb{1} ; m_{1}, n_{1}\right)\right)=\left(m_{1}, n_{1}\right)\left(a_{1}, a_{2}\right)\left(m_{1}, n_{1}\right) \\
& =\left(m_{1}+(-1)^{n_{1}} a_{1}, n_{1}+a_{2}\right)\left(m_{1}, n_{1}\right)=\left(m_{1}+(-1)^{n_{1}} a_{1}+(-1)^{n_{1}+a_{2}} m_{1}, 2 n_{1}+a_{2}\right) .
\end{aligned}
$$

Da igualdade da segunda coordenada de (4.17), temos que $a_{2}=-2 n_{1}+2 i$ e portanto, $a_{2}$ é par. Segue da igualdade da primeira coordenada de (4.17) que $a_{1}= \begin{cases}-2 m_{1}, & \text { se } n_{1} \text { é par; } \\ 0, & \text { se } n_{1} \text { é ímpar. }\end{cases}$

Por $(i)$, pela comutatividade do diagrama (4.5) e pelas Observações 4.3.1 e 4.3.3 temos que

$$
\begin{aligned}
\left(p_{1}\right)_{\#}\left(a l_{\sigma}(b)\right) & =\left(p_{1}\right)_{\#}(b a) \\
\left(p_{1}\right)_{\#}\left(\left(u^{a_{1}} v^{a_{2}} x ; m_{1}, n_{1}\right) l_{\sigma}\left(u^{b_{1}} v^{b_{2}} y ; j, 1\right)\right) & =\left(p_{1}\right)_{\#}\left(\left(u^{b_{1}} v^{b_{2}} y ; j, 1\right)\left(u^{a_{1}} v^{a_{2}} x ; m_{1}, n_{1}\right)\right) \\
\left(m_{1}, n_{1}\right)\left(b_{1}, b_{2}\right)(j, 1) & =(j, 1)\left(m_{1}, n_{1}\right) \\
\left(m_{1}+(-1)^{n_{1}} b_{1}, n_{1}+b_{2}\right)(j, 1) & =\left(j-m_{1}, 1+n_{1}\right) \\
\left(m_{1}+(-1)^{n_{1}} b_{1}+(-1)^{n_{1}+b_{2}} j, n_{1}+b_{2}+1\right) & =\left(j-m_{1}, 1+n_{1}\right) .
\end{aligned}
$$

Da igualdade da segunda coordenada de (4.18), segue que $b_{2}=0$. Assim, da igualdade da primeira coordenada de (4.18) temos que $b_{1}= \begin{cases}-2 m_{1}, & \text { se } n_{1} \text { é par; } \\ 2 m_{1}-2 j, & \text { se } n_{1} \text { é ímpar. }\end{cases}$ 
Vamos mostrar que se $n_{1}$ é par, então obtemos a equação (4.15) e se $n_{1}$ é ímpar, então obtemos a equação (4.16).

$1^{o}$ caso: Suponhamos $n_{1}$ par. Seja $n \in \mathbb{Z}$ tal que $n_{1}=2 n$ e seja $m=m_{1}$. Temos que

$$
a=\left(u^{-2 m} v^{-4 n+2 i} x ; m, 2 n\right) \quad \text { e } \quad b=\left(u^{-2 m} y ; j, 1\right) .
$$

Vamos calcular $a l_{\sigma}(b)$ e ba. Usaremos fortemente a Observação 4.3.1 e a igualdade (4.6). Temos

$$
\begin{aligned}
a l_{\sigma}(b)= & \left(u^{-2 m} v^{-4 n+2 i} x ; m, 2 n\right) l_{\sigma}\left(u^{-2 m} y ; j, 1\right) \\
= & \left(u^{-2 m} v^{-4 n+2 i} x ; m, 2 n\right)\left(\left(B u^{-1}\right)^{-2 m} B^{2 m} ;-2 m, 0\right)(\rho(y) ; 0,0)(B ; j, 1) \\
= & \left(u^{-2 m} v^{-4 n+2 i} x \theta(m, 2 n)\left(\left(B u^{-1}\right)^{-2 m} B^{2 m}\right) ;-m, 2 n\right)(\rho(y) B ; j, 1) \\
= & \left(u^{-2 m} v^{-4 n+2 i} x\left(B\left(B^{m} u B^{-m}\right)^{-1}\right)^{-2 m} B^{2 m} \theta(-m, 2 n)(\rho(y) B) ;-m+j, 2 n+1\right) \\
= & \left(u^{-2 m} v^{-4 n+2 i} x\left(B B^{m} u^{-1} B^{-m}\right)^{-2 m} B^{2 m} \theta(-m, 2 n)(\rho(y)) B ;-m+j, 2 n+1\right) \\
= & \left(u^{-2 m} v^{-4 n+2 i} x B^{m}\left(B u^{-1}\right)^{-2 m} B^{-m} B^{2 m} \theta(-m, 2 n)(\rho(y)) B ;-m+j, 2 n+1\right) \\
= & \left(u^{-2 m} v^{-4 n+2 i} x B^{m}\left(B u^{-1}\right)^{-2 m} B^{m} \theta(-m, 2 n)(\rho(y)) B ;-m+j, 2 n+1\right) \\
= & \left(\left(v^{-4 n+2 i} v^{4 n-2 i}\right) u^{-2 m} v^{-4 n+2 i}\left(u^{2 m} u^{-2 m}\right) x B^{m}\left(u^{2 m} u^{-2 m}\right)\left(B u^{-1}\right)^{-2 m} B^{m} \theta(-m, 2 n)(\rho(y))\right. \\
& B ;-m+j, 2 n+1) \\
= & \left(v^{-4 n+2 i}\left[v^{4 n-2 i}, u^{-2 m}\right]\left(u^{-2 m} x B^{m} u^{2 m}\right)\left(u^{-2 m}\left(B u^{-1}\right)^{-2 m}\right) B^{m} \theta(-m, 2 n)(\rho(y)) B ;\right. \\
= & -m+j, 2 n+1) \\
& \left(v^{-4 n+2 i} O_{2 n-i,-2 m} c_{0,-2 m}(x) B_{0,-2 m}^{m}{ }_{-2 m} B_{0,0}^{m} \theta(-m, 2 n)(\rho(y)) B_{0,0} ;-m+j, 2 n+1\right)
\end{aligned}
$$

e

$$
\begin{aligned}
b a= & \left(u^{-2 m} y ; j, 1\right)\left(u^{-2 m} v^{-4 n+2 i} x ; m, 2 n\right)=\left(u^{-2 m} y \theta(j, 1)\left(u^{-2 m} v^{-4 n+2 i} x\right) ; j-m, 2 n+1\right) \\
= & \left(u^{-2 m} y\left(B^{j-1} u^{-1} B^{-j+1}\right)^{-2 m}\left(B^{j} v u^{-2 j} B^{-j+1}\right)^{-4 n+2 i} \theta(j, 1)(x) ; j-m, 2 n+1\right) \\
= & \left.\left(u^{-2 m} y B^{j-1} u^{2 m} B^{-j+1} B^{j}\left(v u^{-2 j} B\right)^{-4 n+2 i} B^{-j} \theta(j, 1)(x) ; j-m, 2 n+1\right)\right) \\
= & \left(\left(v^{-4 n+2 i} v^{4 n-2 i}\right) u^{-2 m} y B^{j-1} u^{2 m}\left(v^{-4 n+2 i} v^{4 n-2 i}\right) B\left(v^{-4 n+2 i} v^{4 n-2 i}(v B)^{-4 n+2 i}(v B)^{4 n-2 i}\right)\right. \\
& \left.\left(v u^{-2 j} B\right)^{-4 n+2 i} B^{-j} \theta(j, 1)(x) ; j-m, 2 n+1\right) \\
= & \left(v ^ { - 4 n + 2 i } ( v ^ { 4 n - 2 i } u ^ { - 2 m } y B ^ { j - 1 } u ^ { 2 m } v ^ { - 4 n + 2 i } ) ( v ^ { 4 n - 2 i } B v ^ { - 4 n + 2 i } ) ( v ^ { 4 n - 2 i } ( v B ) ^ { - 4 n + 2 i } ) \left((v B)^{4 n-2 i}\right.\right. \\
& \left.\left.\left(v u^{-2 j} B\right)^{-4 n+2 i}\right) B^{-j} \theta(j, 1)(x) ; j-m, 2 n+1\right) \\
= & \left(v^{-4 n+2 i} c_{4 n-2 i,-2 m}(y) B_{4 n-2 i,-2 m}^{j-1} B_{4 n-2 i, 0} I_{2 n-i} D_{2 n-i,-2 j} B_{0,0}^{-j} \theta(j, 1)(x) ; j-m, 2 n+1\right) .
\end{aligned}
$$

Notemos que a primeira coordenada de $a l_{\sigma}$ e a primeira coordenada de $b a$ estão escritas na forma normal dada pela Proposição 4.3.4. Como $a l_{\sigma}(b)=b a$, obtemos a seguinte igualdade em $\overline{\left\langle\sigma^{2}\right\rangle}$ :

$$
\begin{gathered}
O_{2 n-i,-2 m} c_{0,-2 m}(x) B_{0,-2 m}^{m} T_{-2 m} B_{0,0}^{m} \theta(-m, 2 n)(\rho(y)) B_{0,0} \\
=c_{4 n-2 i,-2 m}(y) B_{4 n-2 i,-2 m}^{j-1} B_{4 n-2 i, 0} I_{2 n-i} D_{2 n-i,-2 j} B_{0,0}^{-j} \theta(j, 1)(x) .
\end{gathered}
$$

Por um abuso de notação, vamos denotar a imagem dos elementos $x$ e $y$ no abelianizado de $\overline{\left\langle\sigma^{2}\right\rangle}$ também por $x$ e $y$, respectivamente. Logo, utilizando a Observação 4.3.2 e a Proposição 4.3.6, obtemos a seguinte equação em ${\overline{\left\langle\sigma^{2}\right\rangle}}_{\mathrm{ab}}$ :

$$
\begin{aligned}
& \left(c_{0,-2 m}\right)_{\mathrm{ab}}(x)-\theta(j, 1)_{\mathrm{ab}}(x)+\left(\left(\theta(-m, 0)_{\mathrm{ab}} \circ \rho_{\mathrm{ab}}\right)(y)-\left(c_{4 n-2 i,-2 m}\right)_{\mathrm{ab}}(y)=\widetilde{I}_{2 n-i}-\widetilde{T}_{-2 m}\right. \\
& +\widetilde{D}_{2 n-i,-2 j}-\widetilde{O}_{2 n-i,-2 m}-(j+m+1) B_{0,0}-m B_{0,-2 m}+B_{4 n-2 i, 0}+(j-1) B_{4 n-2 i,-2 m}
\end{aligned}
$$

Notemos que obtemos a igualdade (4.15), o que encerra esta parte da demonstração. 
$2^{o}$ caso: Suponhamos $n_{1}$ impar. Seja $n \in \mathbb{Z}$ tal que $n_{1}=2 n+1$ e seja $m=m_{1}$. Temos que

$$
a=\left(v^{-4 n+2 i-2} x ; m, 2 n+1\right) \quad \text { e } \quad b=\left(u^{2 m-2 j} y ; j, 1\right) .
$$

Vamos calcular $a l_{\sigma}(b)$ e $b a$. Usaremos fortemente a Observação 4.3.1 e a igualdade (4.6). Temos

$$
\begin{aligned}
a l_{\sigma}(b)= & \left(v^{-4 n+2 i-2} x ; m, 2 n+1\right) l_{\sigma}\left(u^{2 m-2 j} y ; j, 1\right) \\
= & \left(v^{-4 n+2 i-2} x ; m, 2 n+1\right)\left(\left(B u^{-1}\right)^{2 m-2 j} B^{-2 m+2 j} ; 2 m-2 j, 0\right)(\rho(y) ; 0,0)(B ; j, 1) \\
= & \left(v^{-4 n+2 i-2} x \theta(m, 2 n+1)\left(\left(B u^{-1}\right)^{2 m-2 j} B^{-2 m+2 j}\right) ;-m+2 j, 2 n+1\right)(\rho(y) B ; j, 1) \\
= & \left(v^{-4 n+2 i-2} x\left(B^{-1}\left(B^{m-1} u^{-1} B^{-m+1}\right)^{-1}\right)^{2 m-2 j} B^{2 m-2 j} \theta(-m+2 j, 2 n+1)(\rho(y) B) ;\right. \\
& -m+j, 2 n+2) \\
= & \left(v^{-4 n+2 i-2} x\left(B^{-1} B^{m-1} u B^{-m+1}\right)^{2 m-2 j} B^{2 m-2 j} \theta(-m+2 j, 2 n+1)(\rho(y)) B^{-1} ;\right. \\
& -m+j, 2 n+2) \\
= & \left(v^{-4 n+2 i-2} x B^{m-1}\left(B^{-1} u\right)^{2 m-2 j} B^{-m+1} B^{2 m-2 j} \theta(-m+2 j, 2 n+1)(\rho(y)) B^{-1} ;\right. \\
& -m+j, 2 n+2) \\
= & \left(v^{-4 n+2 i-2}\left(u^{2 m-2 j} u^{-2 m+2 j}\right) x B^{m-1}\left(u^{2 m-2 j} u^{-2 m+2 j}\right)\left(B^{-1} u\right)^{2 m-2 j} B^{-m+1} B^{2 m-2 j}\right. \\
& \left.\theta(-m+2 j, 2 n+1)(\rho(y)) B^{-1} ;-m+j, 2 n+2\right) \\
= & \left(u^{2 m-2 j} v^{-4 n+2 i-2}\left[v^{4 n-2 i+2}, u^{-2 m+2 j}\right]\left(u^{-2 m+2 j} x B^{m-1} u^{2 m-2 j}\right)\left(u^{-2 m+2 j}\left(B^{-1} u\right)^{2 m-2 j}\right)\right. \\
& \left.B^{m-2 j+1} \theta(-m+2 j, 2 n+1)(\rho(y)) B^{-1} ;-m+j ; 2 n+2\right) \\
= & \left(u^{2 m-2 j} v^{-4 n+2 i-2} O_{2 n-i+1,-2 m+2 j} c_{0,-2 m+2 j}(x) B_{0,-2 m+2 j}^{m-1} W_{-2 m+2 j} B_{0,0}^{m-2 j+1}\right. \\
& \left.\theta(-m+2 j, 2 n+1)(\rho(y)) B_{0,0}^{-1} ;-m+j ; 2 n+2\right)
\end{aligned}
$$

e

$$
\begin{aligned}
b a= & \left(u^{2 m-2 j} y ; j, 1\right)\left(v^{-4 n+2 i-2} x ; m, 2 n+1\right) \\
= & \left(u^{2 m-2 j} y\left(B^{j} v u^{-2 j} B^{-j+1}\right)^{-4 n+2 i-2} \theta(j, 1)(x) ; j-m, 2 n+2\right) \\
= & \left(u^{2 m-2 j} y B^{j}\left(v u^{-2 j} B\right)^{-4 n+2 i-2} B^{-j} \theta(j, 1)(x) ; j-m, 2 n+2\right) \\
= & \left(u^{2 m-2 j}\left(v^{-4 n+2 i-2} v^{4 n-2 i+2}\right) y B^{j}\left(v^{-4 n+2 i-2} v^{4 n-2 i+2}(v B)^{-4 n+2 i-2}(v B)^{4 n-2 i+2}\right)\right. \\
& \left.\left(v u^{-2 j} B\right)^{-4 n+2 i-2} B^{-j} \theta(j, 1)(x) ; j-m, 2 n+2\right) \\
= & \left(u^{2 m-2 j} v^{-4 n+2 i-2}\left(v^{4 n-2 i+2} y B^{j} v^{-4 n+2 i-2}\right)\left(v^{4 n-2 i+2}(v B)^{-4 n+2 i-2}\right)\right. \\
& \left.\left((v B)^{4 n-2 i+2}\left(v u^{-2 j} B\right)^{-4 n+2 i-2}\right) B^{-j} \theta(j, 1)(x) ; j-m, 2 n+2\right) \\
= & \left(u^{2 m-2 j} v^{-4 n+2 i-2} c_{4 n-2 i+2,0}(y) B_{4 n-2 i+2,0}^{j} I_{2 n-i+1} D_{2 n-i+1,-2 j} B_{0,0}^{-j} \theta(j, 1)(x) ; j-m, 2 n+2\right) .
\end{aligned}
$$

Notemos que a primeira coordenada de $a l_{\sigma}$ e a primeira coordenada de $b a$ estão escritas na forma normal dada pela Proposição 4.3.4. Como $a l_{\sigma}(b)=b a$, obtemos a seguinte igualdade em $\overline{\left\langle\sigma^{2}\right\rangle}$ :

$$
\begin{aligned}
& O_{2 n-i+1,-2 m+2 j} c_{0,-2 m+2 j}(x) B_{0,-2 m+2 j}^{m-1} W_{-2 m+2 j} B_{0,0}^{m-2 j+1} \theta(-m+2 j, 2 n+1)(\rho(y)) B_{0,0}^{-1} \\
= & c_{4 n-2 i+2,0}(y) B_{4 n-2 i+2,0}^{j} I_{2 n-i+1} D_{2 n-i+1,-2 j} B_{0,0}^{-j} \theta(j, 1)(x) .
\end{aligned}
$$

Por um abuso de notação, vamos denotar a imagem dos elementos $x$ e $y$ no abelianizado de $\overline{\left\langle\sigma^{2}\right\rangle}$ também por $x$ e $y$, respectivamente. Logo, utilizando a Observação 4.3.2 e a Proposição 4.3.6, obtemos a seguinte equação em ${\overline{\left\langle\sigma^{2}\right\rangle}}_{\mathrm{ab}}$ :

$$
\begin{aligned}
& \left(c_{0,-2 m+2 j}\right)_{\mathrm{ab}}(x)-\theta(j, 1)_{\mathrm{ab}}(x)+\left(\theta(-m+2 j, 1)_{\mathrm{ab}} \circ \rho_{\mathrm{ab}}\right)(y)-\left(c_{4 n-2 i+2,0}\right)_{\mathrm{ab}}(y) \\
& =\widetilde{I}_{2 n-i+1}-\widetilde{W}_{-2 m+2 j}+\widetilde{D}_{2 n-i+1,-2 j}+(j-m) B_{0,0}+(-m+1) B_{0,-2 m+2 j}+j B_{4 n-2 i+2,0} .
\end{aligned}
$$

Notemos que obtemos a igualdade (4.16), o que encerra a demonstração.

Lema 4.4.17. Nẫo existem elementos $x, y \in{\overline{\left\langle\sigma^{2}\right\rangle}}_{\mathrm{ab}}$ e $(m, n) \in \mathbb{Z} \rtimes \mathbb{Z}$ que satisfazem a equação 


\section{(4.15) ou a equação (4.16) do Lema 4.4.16.}

Demonstração. Vamos fazer a prova por contradição. Suponhamos então que existam elementos $x, y \in{\overline{\left\langle\sigma^{2}\right\rangle}}_{\mathrm{ab}}$ e $(m, n) \in \mathbb{Z} \rtimes \mathbb{Z}$ que satisfazem uma das seguintes equações:

$$
\begin{aligned}
\quad \mu_{1}(x)+\nu_{1}(y)= & \widetilde{I}_{2 n-i}-\widetilde{T}_{-2 m}+\widetilde{D}_{2 n-i,-2 j}-\widetilde{O}_{2 n-i,-2 m} \\
& -(j+m+1) B_{0,0}-m B_{0,-2 m}+B_{4 n-2 i, 0}+(j-1) B_{4 n-2 i,-2 m} \\
\text { (2) } \mu_{2}(x)+\nu_{2}(y)= & \widetilde{I}_{2 n-i+1}-\widetilde{W}_{-2 m+2 j}+\widetilde{D}_{2 n-i+1,-2 j} \\
& +(j-m) B_{0,0}+(-m+1) B_{0,-2 m+2 j}+j B_{4 n-2 i+2,0},
\end{aligned}
$$

sendo

$$
\begin{aligned}
\mu_{1}(x) & =\left(c_{0,-2 m}\right)_{\mathrm{ab}}(x)-\theta(j, 1)_{\mathrm{ab}}(x) \\
\nu_{1}(y) & =\left(\left(\theta(-m, 0)_{\mathrm{ab}} \circ \rho_{\mathrm{ab}}\right)(y)-\left(c_{4 n-2 i,-2 m}\right)_{\mathrm{ab}}(y)\right. \\
\mu_{2}(x) & =\left(c_{0,-2 m+2 j}\right)_{\mathrm{ab}}(x)-\theta(j, 1)_{\mathrm{ab}}(x) \\
\nu_{2}(y) & =\left(\theta(-m+2 j, 1)_{\mathrm{ab}} \circ \rho_{\mathrm{ab}}\right)(y)-\left(c_{4 n-2 i+2,0}\right)_{\mathrm{ab}}(y) .
\end{aligned}
$$

Seja

$$
\epsilon:{\overline{\left\langle\sigma^{2}\right\rangle}}_{\mathrm{ab}}=\bigoplus_{s, r \in \mathbb{Z}} \mathbb{Z}\left[B_{s, r}\right] \longrightarrow \mathbb{Z}_{2}
$$

o homomorfismo definido nos elementos da base por

$$
\epsilon\left(B_{s, r}\right)=\overline{1}
$$

e extendido por linearidade. Notemos que se $z$ é um elemento de $\overline{\left\langle\sigma^{2}\right\rangle}$ ab que se escreve como uma soma par (resp. ímpar) de elementos da base de $\overline{\left\langle\sigma^{2}\right\rangle}$ ab , então $\epsilon(z)=\overline{0}$ (resp. $\overline{1}$ ). Pela Proposição 4.3.6, é fácil ver que para todo $a, p, q \in \mathbb{Z}$, temos

$$
\epsilon=\epsilon \circ \theta(a, 0)_{\mathrm{ab}}=\epsilon \circ \theta(a, 1)_{\mathrm{ab}}=\epsilon \circ \rho_{\mathrm{ab}}=\epsilon \circ\left(c_{p, q}\right)_{\mathrm{ab}} .
$$

Logo,

$$
\epsilon\left(\mu_{1}(x)+\nu_{1}(y)\right)=\epsilon\left(\mu_{2}(x)+\nu_{2}(y)\right)=\overline{0} .
$$

Pelos Proposição 4.3.7, os elementos $\widetilde{I}_{2 n-i}, \widetilde{I}_{2 n-i+1}$ e $\widetilde{O}_{2 n-i,-2 m}$ se escrevem como uma soma par de elementos da base de $\overline{\left\langle\sigma^{2}\right\rangle}$ ab , e portanto

$$
\epsilon\left(\widetilde{I}_{2 n-i}\right)=\epsilon\left(\widetilde{I}_{2 n-i+1}\right)=\epsilon\left(\widetilde{O}_{2 n-i,-2 m}\right)=\overline{0} .
$$

Como $-2 m,-2 m+2 j$ e $-2 j$ são pares, segue pela Proposição 4.3 .7 que

$$
\epsilon\left(\widetilde{T}_{-2 m}\right)=\epsilon\left(\widetilde{W}_{-2 m+2 j}\right)=\epsilon\left(\widetilde{D}_{2 n-i,-2 j}\right)=\epsilon\left(\widetilde{D}_{2 n-i+1,-2 j}\right)=\overline{0} .
$$

Assim, por (1) temos que

$$
\begin{aligned}
\overline{0} & =\epsilon\left(-(j+m+1) B_{0,0}-m B_{0,-2 m}+B_{4 n-2 i, 0}+(j-1) B_{4 n-2 i,-2 m}\right) \\
& =\overline{j+m+1}+\overline{-m}+\overline{1}+\overline{j-1}=\overline{1},
\end{aligned}
$$

o que é um absurdo. Portanto, não existem elementos $x, y \in{\overline{\left\langle\sigma^{2}\right\rangle}}_{\mathrm{ab}}$ e $(m, n) \in \mathbb{Z} \rtimes \mathbb{Z}$ que satisfazem a equação (4.15). Por (2) temos que

$$
\overline{0}=\epsilon\left((j-m) B_{0,0}+(-m+1) B_{0,-2 m+2 j}+j B_{4 n-2 i+2,0}\right)=\overline{j-m}+\overline{-m+1}+\bar{j}=\overline{1},
$$

o que também é um absurdo. Portanto, não existem elementos $x, y \in{\overline{\left\langle\sigma^{2}\right\rangle}}_{\mathrm{ab}}$ e $(m, n) \in \mathbb{Z} \rtimes \mathbb{Z}$ que 
satisfazem a equação (4.16), o que encerra a demonstração.

Demonstração da Proposição 4.4.5. Queremos mostrar que se $\alpha \in\left[\mathbb{T}^{2}, * ; \mathbb{K}^{2}, *\right]$ é uma classe de homotopia pontuada cujo homomorfismo $h_{\alpha}=\Gamma(\alpha): \pi_{1}\left(\mathbb{T}^{2}\right) \rightarrow \pi_{1}\left(\mathbb{K}^{2}\right)$ é tal que

$$
h_{\alpha}(1,0)=\left(0,2 s_{1}\right) \quad \text { e } \quad h_{\alpha}(0,1)=\left(j, 2 s_{2}+1\right),
$$

para algum $j \in\{0,1\}$ e para algum $s_{1}, s_{2} \in \mathbb{Z}$, então $\alpha$ tem a propriedade de Borsuk-Ulam. Pelo Corolário 4.4.12, para que este resultado fique provado basta mostrarmos que se $\alpha \in\left[\mathbb{T}^{2}, * ; \mathbb{K}^{2}, *\right]$ é uma classe de homotopia pontuada cujo homomorfismo $h_{\alpha}=\Gamma(\alpha): \pi_{1}\left(\mathbb{T}^{2}\right) \rightarrow \pi_{1}\left(\mathbb{K}^{2}\right)$ é tal que

$$
h_{\alpha}(1,0)=(0,2 i) \quad \text { e } \quad h_{\alpha}(0,1)=(j, 1),
$$

para algum $i, j \in\{0,1\}$, então $\alpha$ tem a propriedade de Borsuk-Ulam. Mas este fato segue diretamente dos Lemas 4.4.16 e 4.4.17.

\subsubsection{Prova das Proposições 4.4.8 e 4.4.10}

O objetivo desta sub-seção é demonstrar as Proposições 4.4 .8 e 4.4.10. Vamos primeiramente enunciar vários resultados preliminares. Usaremos fortemente as notações e os resultados da Seção 4.3.

Lema 4.4.18. Seja $\alpha \in\left[\mathbb{T}^{2}, * ; \mathbb{K}^{2}, *\right]$ uma classe de homotopia cujo homomorfismo $h_{\alpha}=\Gamma(\alpha): \pi_{1}\left(\mathbb{T}^{2}\right) \rightarrow \pi_{1}\left(\mathbb{K}^{2}\right)$ é tal que

$$
h_{\alpha}(1,0)=\left(r_{1}, 2\right) \quad e \quad h_{\alpha}(0,1)=\left(r_{2}, 0\right),
$$

sendo $r_{1}$ e $r_{2}$ números inteiros. Utilizando a notação da Proposição 4.3.7, temos que se $\alpha$ não tem a propriedade de Borsuk-Ulam, então existem elementos $x, y \in{\overline{\left\langle\sigma^{2}\right\rangle_{\mathrm{ab}}}}$ e $(m, n) \in \mathbb{Z} \rtimes \mathbb{Z}$ que satisfazem uma das seguintes equaçôes em $\overline{\left\langle\sigma^{2}\right\rangle} \mathrm{ab}=\bigoplus_{s, r \in \mathbb{Z}}\left[B_{s, r}\right]$ :

(1) $\mu_{1}(x)+\nu_{1}(y)=\widetilde{J}_{2 n-1,-2 r_{2}}-r_{2} B_{0,0}+r_{2} B_{4 n-2,2 m-r_{1}}$

(2) $\mu_{2}(x)+\nu_{2}(y)=\widetilde{J}_{2 n,-2 r_{2}}-\widetilde{O}_{2 n, 2 r_{2}}-\widetilde{W}_{2 r_{2}}+\left(r_{2}+m-1\right) B_{0,0}+(1-m) B_{0,2 r_{2}}+r_{2} B_{4 n, r_{1}}$ sendo $\mu_{1}, \nu_{1}, \mu_{2}, \nu_{2}:{\overline{\left\langle\sigma^{2}\right\rangle}}_{\mathrm{ab}}=\bigoplus_{s, r \in \mathbb{Z}}\left[B_{s, r}\right] \rightarrow{\overline{\left\langle\sigma^{2}\right\rangle}}_{\mathrm{ab}}$ homomorfismos que são definidos nos elementos da base do seguinte modo:

$$
\begin{aligned}
& \mu_{1}\left(B_{s, r}\right)=\left\{\begin{array}{l}
0, \text { se s é par } \\
B_{s, r}-B_{s, r-2 r_{2}}, \text { se s é impar }
\end{array}\right. \\
& \nu_{1}\left(B_{s, r}\right)=\left\{\begin{array}{l}
B_{-s,-r}-B_{s+4 n-2, r+2 m-r_{1}}, \text { se s é par } \\
-B_{-s, r-2 m}-B_{s+4 n-2, r-2 m+r_{1}} \text {, se sé ímpar }
\end{array}\right. \\
& \mu_{2}\left(B_{s, r}\right)=\left\{\begin{array}{l}
B_{s, r+2 r_{2}}-B_{s, r}, \text { se s é par } \\
0, \text { se s é impar }
\end{array}\right. \\
& \nu_{2}\left(B_{s, r}\right)=\left\{\begin{array}{l}
-B_{-s, r}-B_{s+4 n, r+r_{1}}, \text { se s é par } \\
B_{-s,-r-4 r_{2}-2 m}-B_{s+4 n, r-r_{1}}, \text { se s é ímpar. }
\end{array}\right.
\end{aligned}
$$

Demonstração. Pela Proposição 4.4.11, como $\alpha$ não tem a propriedade de Borsuk-Ulam, existem elementos de $P_{2}\left(\mathbb{K}^{2}\right)$

$$
a=\left(w ; m_{1}, n_{1}\right) \quad \text { e } \quad b=\left(z ; m_{2}, n_{2}\right)
$$

tais que 

(i) $a l_{\sigma}(b)=b a$
(ii) $\left(p_{1}\right)_{\#}\left(a l_{\sigma}(a)\right)=\left(r_{1}, 2\right)$
(iii) $\left(p_{1}\right)_{\#}(b)=\left(r_{2}, 0\right)$.

Pela Observação 4.3 .1 e por $(i i i)$, temos que $\left(m_{2}, n_{2}\right)=\left(r_{2}, 0\right)$. Pela Proposição 4.3.4, existem $x, y \in \overline{\left\langle\sigma^{2}\right\rangle}$ tais que $w=u^{a_{1}} v^{a_{2}} x$ e $z=u^{b_{1}} v^{b_{2}} y$, sendo $g(w)=\left(a_{1}, a_{2}\right)$ e $g(z)=\left(b_{1}, b_{2}\right)$. Logo,

$$
a=\left(u^{a_{1}} v^{a_{2}} x ; m_{1}, n_{1}\right) \quad \text { e } \quad b=\left(u^{b_{1}} v^{b_{2}} y ; r_{2}, 0\right) .
$$

Usando a Observação 4.3.1, a comutatividade do diagrama (4.5) e (ii), temos que

$$
\begin{aligned}
\left(r_{1}, 2\right) & =\left(p_{1}\right)_{\#}\left(a l_{\sigma}(a)\right)=\left(p_{1}\right)_{\#}\left(u^{a_{1}} v^{a_{2}} x ; m_{1}, n_{1}\right)\left(p_{1}\right)_{\#}\left(l_{\sigma}\left(u^{a_{1}} v^{a_{2}} x ; m_{1}, n_{1}\right)\right) \\
& =\left(m_{1}, n_{1}\right)\left(p_{1}\right)_{\#}\left(l_{\sigma}\left(u^{a_{1}} v^{a_{2}} x ; 0,0\right)\right)\left(p_{1}\right)_{\#}\left(l_{\sigma}\left(\mathbb{1} ; m_{1}, n_{1}\right)\right) \\
& =\left(m_{1}, n_{1}\right)\left(a_{1}, a_{2}\right)\left(m_{1}, n_{1}\right)=\left(m_{1}+(-1)^{n_{1}} a_{1}, n_{1}+a_{2}\right)\left(m_{1}, n_{1}\right) \\
& =\left(m_{1}+(-1)^{n_{1}} a_{1}+(-1)^{n_{1}+a_{2}} m_{1}, 2 n_{1}+a_{2}\right) .
\end{aligned}
$$

Da igualdade da segunda coordenada de (4.19), temos que $a_{2}=-2 n_{1}+2$ e portanto, $a_{2}$ é par. Segue da igualdade da primeira coordenada de (4.19) que $a_{1}= \begin{cases}-2 m_{1}+r_{1}, & \text { se } n_{1} \text { é par; } \\ -r_{1}, & \text { se } n_{1} \text { é ímpar. }\end{cases}$

Por $(i)$ e pela comutatividade do diagrama (4.5), temos que

$$
\begin{aligned}
\left(p_{1}\right)_{\#}\left(a l_{\sigma}(b)\right) & =\left(p_{1}\right)_{\#}(b a) \\
\left(p_{1}\right)_{\#}\left(\left(u^{a_{1}} v^{a_{2}} x ; m_{1}, n_{1}\right) l_{\sigma}\left(u^{b_{1}} v^{b_{2}} y ; r_{2}, 0\right)\right) & =\left(p_{1}\right)_{\#}\left(\left(u^{b_{1}} v^{b_{2}} y ; r_{2}, 0\right)\left(u^{a_{1}} v^{a_{2}} x ; m_{1}, n_{1}\right)\right) \\
\left(m_{1}, n_{1}\right)\left(b_{1}, b_{2}\right)\left(r_{2}, 0\right) & =\left(r_{2}, 0\right)\left(m_{1}, n_{1}\right) \\
\left(m_{1}+(-1)^{n_{1}} b_{1}, n_{1}+b_{2}\right)\left(r_{2}, 0\right) & =\left(r_{2}+m_{1}, n_{1}\right) \\
\left(m_{1}+(-1)^{n_{1}} b_{1}+(-1)^{n_{1}+b_{2}} r_{2}, n_{1}+b_{2}\right) & =\left(r_{2}+m_{1}, n_{1}\right) .
\end{aligned}
$$

Da igualdade da segunda coordenada de (4.20), segue que $b_{2}=0$. Assim, da igualdade da primeira coordenada de (4.20) temos que $b_{1}= \begin{cases}0, & \text { se } n_{1} \text { é par; } \\ -2 r_{2}, & \text { se } n_{1} \text { é ímpar. }\end{cases}$

Vamos mostrar que se $n_{1}$ é par, então obtemos a equação (1) e se $n_{1}$ é ímpar, então obtemos a equação (2).

$1^{o}$ caso: Suponhamos $n_{1}$ par. Seja $n \in \mathbb{Z}$ tal que $n_{1}=2 n$ e seja $m=m_{1}$. Temos que

$$
a=\left(u^{-2 m+r_{1}} v^{-4 n+2} x ; m, 2 n\right) \quad \text { e } \quad b=\left(y ; r_{2}, 0\right) .
$$

Vamos calcular $a l_{\sigma}(b)$ e $b a$. Usaremos fortemente a Observação 4.3 .1 e a igualdade (4.6). Temos

$$
\begin{aligned}
a l_{\sigma}(b) & =\left(u^{-2 m+r_{1}} v^{-4 n+2} x ; m, 2 n\right) l_{\sigma}\left(y ; r_{2}, 0\right) \\
& =\left(u^{-2 m+r_{1}} v^{-4 n+2} x ; m, 2 n\right)(\rho(y) ; 0,0)\left(\mathbb{1} ; r_{2}, 0\right) \\
& =\left(u^{-2 m+r_{1}} v^{-4 n+2} x \theta(m, 2 n)(\rho(y)) ; m+r_{2}, 2 n\right)
\end{aligned}
$$


e

$$
\begin{aligned}
b a= & \left(y ; r_{2}, 0\right)\left(u^{-2 m+r_{1}} v^{-4 n+2} x ; m, 2 n\right)=\left(y \theta\left(r_{2}, 0\right)\left(u^{-2 m+r_{1}} v^{-4 n+2} x\right) ; r_{2}+m, 2 n\right) \\
= & \left(y\left(B^{r_{2}} u B^{-r_{2}}\right)^{-2 m+r_{1}}\left(B^{r_{2}} v u^{-2 r_{2}} B^{-r_{2}}\right)^{-4 n+2} \theta\left(r_{2}, 0\right)(x) ; r_{2}+m, 2 n\right) \\
= & \left(y B^{r_{2}} u^{-2 m+r_{1}} B^{-r_{2}} B^{r_{2}}\left(v u^{-2 r_{2}}\right)^{-4 n+2} B^{-r_{2}} \theta\left(r_{2}, 0\right)(x) ; r_{2}+m, 2 n\right) \\
= & \left(\left(u^{-2 m+r_{1}} v^{-4 n+2} v^{4 n-2} u^{2 m-r_{1}}\right) y B^{r_{2}} u^{-2 m+r_{1}}\left(v^{-4 n+2} v^{4 n-2}\right)\left(v u^{-2 r_{2}}\right)^{-4 n+2} B^{-r_{2}} \theta\left(r_{2}, 0\right)(x) ;\right. \\
& \left.r_{2}+m, 2 n\right) \\
= & \left(u^{-2 m+r_{1}} v^{-4 n+2}\left(v^{4 n-2} u^{2 m-r_{1}} y B^{r_{2}} u^{-2 m+r_{1}} v^{-4 n+2}\right)\left(v^{4 n-2}\left(v u^{-2 r_{2}}\right)^{-4 n+2}\right) B^{-r_{2}} \theta\left(r_{2}, 0\right)(x) ;\right. \\
& \left.r_{2}+m, 2 n\right) \\
= & \left(u^{-2 m+r_{1}} v^{-4 n+2} c_{4 n-2,2 m-r_{1}}(y) B_{4 n-2,2 m-r_{1}}^{r_{2}} J_{2 n-1,-2 r_{2}} B_{0,0}^{-r_{2}} \theta\left(r_{2}, 0\right)(x) ; r_{2}+m, 2 n\right) .
\end{aligned}
$$

Notemos que a primeira coordenada de $a l_{\sigma}$ e a primeira coordenada de $b a$ estão escritas na forma normal dada pela Proposição 4.3.4. Como $a l_{\sigma}(b)=b a$, obtemos a seguinte igualdade em $\overline{\left\langle\sigma^{2}\right\rangle}$ :

$$
x \theta(m, 2 n)(\rho(y))=c_{4 n-2,2 m-r_{1}}(y) B_{4 n-2,2 m-r_{1}}^{r_{2}} J_{2 n-1,-2 r_{2}} B_{0,0}^{-r_{2}} \theta\left(r_{2}, 0\right)(x) .
$$

Por um abuso de notação, vamos denotar a imagem dos elementos $x$ e $y$ no abelianizado de $\overline{\left\langle\sigma^{2}\right\rangle}$ também por $x$ e $y$, respectivamente. Logo, utilizando a Observação 4.3.2 e a Proposição 4.3.6, obtemos a seguinte equação em ${\overline{\left\langle\sigma^{2}\right\rangle}}_{\mathrm{ab}}$ :

$x-\theta\left(r_{2}, 0\right)_{\mathrm{ab}}(x)+\left(\theta(m, 0)_{\mathrm{ab}} \circ \rho_{\mathrm{ab}}\right)(y)-\left(c_{4 n-2,2 m-r_{1}}\right)_{\mathrm{ab}}(y)=\widetilde{J}_{2 n-1,-2 r_{2}}-r_{2} B_{0,0}+r_{2} B_{4 n-2,2 m-r_{1}}$.

Vamos mostrar que para cada elemento $B_{s, r} \in \overline{\left\langle\sigma^{2}\right\rangle}$ ab , temos que $B_{s, r}-\theta\left(r_{2}, 0\right)_{\mathrm{ab}}\left(B_{s, r}\right)=\mu_{1}\left(B_{s, r}\right)$ e $\left(\theta(m, 0)_{\mathrm{ab}} \circ \rho_{\mathrm{ab}}\right)\left(B_{s, r}\right)-\left(c_{4 n-2,2 m-r_{1}}\right)_{\mathrm{ab}}\left(B_{s, r}\right)=\nu_{1}\left(B_{s, r}\right)$, o que completará esta parte da demonstração. Utilizaremos a Proposição 4.3.6.

Se $s$ é par, temos que

$$
B_{s, r}-\theta\left(r_{2}, 0\right)_{\mathrm{ab}}\left(B_{s, r}\right)=B_{s, r}-B_{s, r}=0=\mu_{1}\left(B_{s, r}\right)
$$

e

$$
\begin{aligned}
\left(\theta(m, 0)_{\mathrm{ab}} \circ \rho_{\mathrm{ab}}\right)\left(B_{s, r}\right)-\left(c_{4 n-2,2 m-r_{1}}\right)_{\mathrm{ab}}\left(B_{s, r}\right) & =\theta(m, 0)_{\mathrm{ab}}\left(B_{-s,-r}\right)-B_{s+4 n-2, r+2 m-r_{1}} \\
& =B_{-s,-r}-B_{s+4 n-2, r+2 m-r_{1}}=\nu_{1}\left(B_{s, r}\right) .
\end{aligned}
$$

Se $s$ é ímpar, temos que

$$
B_{s, r}-\theta\left(r_{2}, 0\right)_{\mathrm{ab}}\left(B_{s, r}\right)=B_{s, r}-B_{s, r-2 r_{2}}=\mu_{1}\left(B_{s, r}\right)
$$

e

$$
\begin{aligned}
\left(\theta(m, 0)_{\mathrm{ab}} \circ \rho_{\mathrm{ab}}\right)\left(B_{s, r}\right)-\left(c_{4 n-2,2 m-r_{1}}\right)_{\mathrm{ab}}\left(B_{s, r}\right) & =\theta(m, 0)_{\mathrm{ab}}\left(-B_{-s, r}\right)-B_{s+4 n-2, r-2 m+r_{1}} \\
& =-B_{-s, r-2 m}-B_{s+4 n-2, r-2 m+r_{1}}=\nu_{1}\left(B_{s, r}\right) .
\end{aligned}
$$

$2^{o}$ caso: Suponhamos $n_{1}$ impar. Seja $n \in \mathbb{Z}$ tal que $n_{1}=2 n+1$ e seja $m=m_{1}$. Temos que

$$
a=\left(u^{-r_{1}} v^{-4 n} x ; m, 2 n+1\right) \quad \text { e } \quad b=\left(u^{-2 r_{2}} y ; r_{2}, 0\right) .
$$


Vamos calcular $a l_{\sigma}(b)$ e $b a$. Usaremos fortemente a Observação 4.3 .1 e a igualdade (4.6). Temos

$$
\begin{aligned}
a l_{\sigma}(b)= & \left(u^{-r_{1}} v^{-4 n} x ; m, 2 n+1\right) l_{\sigma}\left(u^{-2 r_{2}} y ; r_{2}, 0\right) \\
= & \left(u^{-r_{1}} v^{-4 n} x ; m, 2 n+1\right)\left(\left(B u^{-1}\right)^{-2 r_{2}} B^{2 r_{2}} ;-2 r_{2}, 0\right)(\rho(y) ; 0,0)\left(\mathbb{1} ; r_{2}, 0\right) \\
= & \left(u^{-r_{1}} v^{-4 n} x \theta(m, 2 n+1)\left(\left(B u^{-1}\right)^{-2 r_{2}} B^{2 r_{2}}\right) ; m+2 r_{2}, 2 n+1\right)\left(\rho(y) ; r_{2}, 0\right) \\
= & \left(u^{-r_{1}} v^{-4 n} x\left(B^{-1}\left(B^{m-1} u^{-1} B^{-m+1}\right)^{-1}\right)^{-2 r_{2}} B^{-2 r_{2}} \theta\left(m+2 r_{2}, 2 n+1\right)(\rho(y)) ;\right. \\
& \left.m+r_{2}, 2 n+1\right) \\
= & \left(u^{-r_{1}} v^{-4 n} x\left(B^{-1} B^{m-1} u B^{-m+1}\right)^{-2 r_{2}} B^{-2 r_{2}} \theta\left(m+2 r_{2}, 2 n+1\right)(\rho(y)) ; m+r_{2}, 2 n+1\right) \\
= & \left(u^{-r_{1}} v^{-4 n} x B^{m-1}\left(B^{-1} u\right)^{-2 r_{2}} B^{-m+1} B^{-2 r_{2}} \theta\left(m+2 r_{2}, 2 n+1\right)(\rho(y)) ; m+r_{2}, 2 n+1\right) \\
= & \left(u^{-r_{1}}\left(u^{-2 r_{2}} v^{-4 n} v^{4 n} u^{2 r_{2}}\right) v^{-4 n}\left(u^{-2 r_{2}} u^{2 r_{2}}\right) x B^{m-1}\left(u^{-2 r_{2}} u^{2 r_{2}}\right)\left(B^{-1} u\right)^{-2 r_{2}} B^{-2 r_{2}-m+1}\right. \\
& \left.\theta\left(m+2 r_{2}, 2 n+1\right)(\rho(y)) ; m+r_{2}, 2 n+1\right) \\
= & \left(u^{-r_{1}-2 r_{2}} v^{-4 n}\left(v^{4 n} u^{2 r_{2}} v^{-4 n} u^{-2 r_{2}}\right)\left(u^{2 r_{2}} x B^{m-1} u^{-2 r_{2}}\right)\left(u^{2 r_{2}}\left(B^{-1} u\right)^{-2 r_{2}}\right) B^{-2 r_{2}-m+1}\right. \\
& \left.\theta\left(m+2 r_{2}, 2 n+1\right)(\rho(y)) ; m+r_{2}, 2 n+1\right) \\
= & \left(u^{-r_{1}-2 r_{2}} v^{-4 n} O_{2 n, 2 r_{2}} c_{0,2 r_{2}}(x) B_{0,2 r_{2}}^{m-1} W_{2 r_{2}} B_{0,0}^{-2 r_{2}-m+1} \theta\left(m+2 r_{2}, 2 n+1\right)(\rho(y)) ;\right. \\
& \left.m+r_{2}, 2 n+1\right)
\end{aligned}
$$

e

$$
\begin{aligned}
b a & =\left(u^{-2 r_{2}} y ; r_{2}, 0\right)\left(u^{-r_{1}} v^{-4 n} x ; m, 2 n+1\right)=\left(u^{-2 r_{2}} y \theta\left(r_{2}, 0\right)\left(u^{-r_{1}} v^{-4 n} x\right) ; m+r_{2}, 2 n+1\right) \\
& =\left(u^{-2 r_{2}} y\left(B^{r_{2}} u B^{-r_{2}}\right)^{-r_{1}}\left(B^{r_{2}} v u^{-2 r_{2}} B^{-r_{2}}\right)^{-4 n} \theta\left(r_{2}, 0\right)(x) ; m+r_{2}, 2 n+1\right) \\
& =\left(u^{-2 r_{2}} y B^{r_{2}} u^{-r_{1}} B^{-r_{2}} B^{r_{2}}\left(v u^{-2 r_{2}}\right)^{-4 n} B^{-r_{2}} \theta\left(r_{2}, 0\right)(x) ; m+r_{2}, 2 n+1\right) \\
& =\left(u^{-2 r_{2}}\left(u^{-r_{1}} v^{-4 n} v^{4 n} u^{r_{1}}\right) y B^{r_{2}} u^{-r_{1}}\left(v^{-4 n} v^{4 n}\right)\left(v u^{-2 r_{2}}\right)^{-4 n} B^{-r_{2}} \theta\left(r_{2}, 0\right)(x) ; m+r_{2}, 2 n+1\right) \\
& =\left(u^{-r_{1}-2 r_{2}} v^{-4 n}\left(v^{4 n} u^{r_{1}} y B^{r_{2}} u^{-r_{1}} v^{-4 n}\right)\left(v^{4 n}\left(v u^{-2 r_{2}}\right)^{-4 n}\right) B^{-r_{2}} \theta\left(r_{2}, 0\right)(x) ; m+r_{2}, 2 n+1\right) \\
& =\left(u^{-r_{1}-2 r_{2}} v^{-4 n} c_{4 n, r_{1}}(y) B_{4 n, r_{1}}^{r_{2}} J_{2 n,-2 r_{2}} B_{0,0}^{-r_{2}} \theta\left(r_{2}, 0\right)(x) ; m+r_{2}, 2 n+1\right) .
\end{aligned}
$$

Notemos que a primeira coordenada de $a l_{\sigma}$ e a primeira coordenada de $b a$ estão escritas na forma normal dada pela Proposição 4.3.4. Como $a l_{\sigma}(b)=b a$, obtemos a seguinte igualdade em $\overline{\left\langle\sigma^{2}\right\rangle}$ :

$O_{2 n, 2 r_{2}} c_{0,2 r_{2}}(x) B_{0,2 r_{2}}^{m-1} W_{2 r_{2}} B_{0,0}^{-2 r_{2}-m+1} \theta\left(m+2 r_{2}, 2 n+1\right)(\rho(y))=c_{4 n, r_{1}}(y) B_{4 n, r_{1}}^{r_{2}} J_{2 n,-2 r_{2}} B_{0,0}^{-r_{2}} \theta\left(r_{2}, 0\right)(x)$.

Por um abuso de notação, vamos denotar a imagem dos elementos $x$ e $y$ no abelianizado de $\overline{\left\langle\sigma^{2}\right\rangle}$ também por $x$ e $y$, respectivamente. Logo, utilizando a Observação 4.3 .2 e a Proposição 4.3.6, obtemos a seguinte equação em ${\overline{\left\langle\sigma^{2}\right\rangle}}_{\mathrm{ab}}$ :

$$
\begin{aligned}
& \left(c_{0,2 r_{2}}\right)_{\mathrm{ab}}(x)-\theta\left(r_{2}, 0\right)_{\mathrm{ab}}(x)+\left(\theta\left(m+2 r_{2}, 1\right)_{\mathrm{ab}} \circ \rho_{\mathrm{ab}}\right)(y)-\left(c_{4 n, r_{1}}\right)_{\mathrm{ab}}(y) \\
= & \widetilde{J}_{2 n,-2 r_{2}}-\widetilde{O}_{2 n, 2 r_{2}}-\widetilde{W}_{2 r_{2}}+\left(r_{2}+m-1\right) B_{0,0}+(1-m) B_{0,2 r_{2}}+r_{2} B_{4 n, r_{1}} .
\end{aligned}
$$

Vamos mostrar que para cada gerador $B_{s, r}$ de ${\overline{\left\langle\sigma^{2}\right\rangle}}_{\mathrm{ab}}$, temos que $\left(c_{0,2 r_{2}}\right)_{\mathrm{ab}}\left(B_{s, r}\right)-\theta\left(r_{2}, 0\right)_{\mathrm{ab}}\left(B_{s, r}\right)=$ $\mu_{2}\left(B_{s, r}\right)$ e $\left(\theta\left(m+2 r_{2}, 1\right)_{\mathrm{ab}} \circ \rho_{\mathrm{ab}}\right)\left(B_{s, r}\right)-\left(c_{4 n, r_{1}}\right)_{\mathrm{ab}}\left(B_{s, r}\right)=\nu_{2}\left(B_{s, r}\right)$, o que completará esta parte da demonstração. Utilizaremos a Proposição 4.3.6.

Se $s$ é par, temos que

$$
\left(c_{0,2 r_{2}}\right)_{\mathrm{ab}}\left(B_{s, r}\right)-\theta\left(r_{2}, 0\right)_{\mathrm{ab}}\left(B_{s, r}\right)=B_{s, r+2 r_{2}}-B_{s, r}=\mu_{2}\left(B_{s, r}\right)
$$

e

$$
\begin{aligned}
\left(\theta\left(m+2 r_{2}, 1\right)_{\mathrm{ab}} \circ \rho_{\mathrm{ab}}\right)\left(B_{s, r}\right)-\left(c_{4 n, r_{1}}\right)_{\mathrm{ab}}\left(B_{s, r}\right) & =\theta\left(m+2 r_{2}, 1\right)_{\mathrm{ab}}\left(B_{-s,-r}\right)-B_{s+4 n, r+r_{1}} \\
& =-B_{-s, r}-B_{s+4 n, r+r_{1}}=\nu_{2}\left(B_{s, r}\right) .
\end{aligned}
$$


Se $s$ é ímpar, temos que

$$
\left(c_{0,2 r_{2}}\right)_{\mathrm{ab}}\left(B_{s, r}\right)-\theta\left(r_{2}, 0\right)_{\mathrm{ab}}\left(B_{s, r}\right)=B_{s, r-2 r_{2}}-B_{s, r-2 r_{2}}=0=\mu_{2}\left(B_{s, r}\right)
$$

$\mathrm{e}$

$$
\begin{aligned}
\left(\theta\left(m+2 r_{2}, 1\right)_{\mathrm{ab}} \circ \rho_{\mathrm{ab}}\right)\left(B_{s, r}\right)-\left(c_{4 n, r_{1}}\right)_{\mathrm{ab}}\left(B_{s, r}\right) & =\theta\left(m+2 r_{2}, 1\right)_{\mathrm{ab}}\left(-B_{-s, r}\right)-B_{s+4 n, r-r_{1}} \\
& =B_{-s,-r-4 r_{2}-2 m}-B_{s+4 n, r-r_{1}}=\nu_{2}\left(B_{s, r}\right) .
\end{aligned}
$$

Em todos os próximos resultados, $r_{2}$ denotará um inteiro não nulo escrito na forma dada pela Observação 4.4.1, isto é,

$$
r_{2}=(-1)^{j\left(r_{2}\right)} 2^{e\left(r_{2}\right)} o\left(r_{2}\right),
$$

sendo $j\left(r_{2}\right) \in\{0,1\}, e\left(r_{2}\right)$ um número inteiro não negativo e $o\left(r_{2}\right)$ um número inteiro ímpar positivo.

Também em todos os enunciados, $r_{1}$ denotará um número inteiro não negativo que satisfaz uma das seguintes condições:
(i) $r_{1}=0$;
(ii) $r_{1}>0$ e $e\left(r_{1}\right)>e\left(r_{2}\right)$.

Por fim, $(m, n)$ denotará um elemento de $\mathbb{Z} \rtimes \mathbb{Z}$.

O objetivo principal do restante desta seção é mostrar que, com estas hipóteses, as equações (1) e (2) do Lema 4.4.18 não têm solução.

Lema 4.4.19. Sejam a e $b$ dois números inteiros positivos. Para cada $\delta \in\{-1,1\}$ e para cada $k \in \mathbb{Z}$ definimos o seguinte sub-conjunto de $\mathbb{Z}$ :

$$
\delta S(a, b)+k=\{\delta x+k \mid 1 \leq x \leq a b, \delta x+k \equiv 0 \bmod a\}
$$

Então, a cardinalidade de $\delta S(a, b)+k$ é $b$.

Demonstração. $1^{o}$ caso: Suponhamos $\delta=1$. Sejam $l \in \mathbb{Z}$ e $r \in\{0,1, \ldots, a-1\}$ tais que $k=l a+r$. É fácil ver que a seguinte função é uma bijeção:

$$
\begin{aligned}
S(a, b)+k & \longrightarrow S(a, b)+r \\
x+k & \longmapsto x+r .
\end{aligned}
$$

Portanto, para que o resultado fique provado, basta mostrarmos que o conjunto $S(a, b)+r$ tem $b$ elementos. Notemos que

$$
S(a, b)+r=\{y \mid 1+r \leq y \leq a b+r, y \equiv 0 \bmod a\} .
$$

Seja

$$
A=\{m a \mid 1 \leq m \leq b\} .
$$

Vamos mostrar que $S(a, b)+r=A$, donde seguirá o resultado. Seja $y \in S(a, b)+r$. Como $y \equiv 0 \bmod a$, então $y=m a$ para algum $m \in \mathbb{Z}$. Como $r \geq 0$, então $1 \leq 1+r \leq y=m a$ e como $a$ é positivo, então $m \geq 1$. Como $r \leq a-1$, então temos que $m a=y \leq a b+r \leq a b+a-1<a b+a=a(b+1)$. Logo, $m<b+1$, ou equivalentemente, $m \leq b$. Assim, temos que $y=m a \in A$, ou seja, mostramos que $S(a, b)+r \subset A$. Seja agora $m a \in A$. É claro que $m a \equiv 0 \bmod a$. Como $m \geq 1$ e $a$ é positivo, então $r<a \leq a m$, ou seja, $1+r \leq m a$. Como $m \leq b$ e $r \geq 0$, então $m a \leq b a \leq a b+r$. Portanto, $m a \in S(a, b)+r$, ou seja, mostramos que $S(a, b)+r \supset A$. Segue que $S(a, b)+r=A$. 
$2^{\circ}$ caso: Suponhamos $\delta=-1$. É fácil ver que a seguinte função é uma bijeção:

$$
\begin{aligned}
-S(a, b)+k & \longrightarrow S(a, b)-k \\
-x+k & \longmapsto x-k .
\end{aligned}
$$

Portanto, pelo $1^{\circ}$ caso, segue que a cardinalidade do conjunto $-S(a, b)+k$ é $b$.

Consideremos o homomorfismo $\varepsilon_{n, r_{2}}: \overline{\left\langle\sigma^{2}\right\rangle_{\mathrm{ab}}}=\bigoplus_{s, r \in \mathbb{Z}} \mathbb{Z}\left[B_{s, r}\right] \rightarrow \mathbb{Z}_{2}$, o qual é definido em cada elemento da base do seguinte modo:

$$
\varepsilon_{n, r_{2}}\left(B_{s, r}\right)=\left\{\begin{array}{l}
\overline{0}, \text { se } s \neq 2 n-1 \\
\overline{0}, \text { se } s=2 n-1 \text { e } r \not \equiv 0 \bmod 2^{e\left(r_{2}\right)+1} \\
\overline{1}, \text { se } s=2 n-1 \text { e } r \equiv 0 \bmod 2^{e\left(r_{2}\right)+1}
\end{array}\right.
$$

Lema 4.4.20. Consideremos o homomorfismo $\mu_{1}:{\overline{\left\langle\sigma^{2}\right\rangle}}_{\mathrm{ab}} \rightarrow{\overline{\left\langle\sigma^{2}\right\rangle}}_{\mathrm{ab}}$ como definido no Lema 4.4.18. Então $\varepsilon_{n, r_{2}} \circ \mu_{1}:{\overline{\left\langle\sigma^{2}\right\rangle}}_{\mathrm{ab}} \rightarrow \mathbb{Z}_{2}$ é identicamente nulo.

Demonstração. Vamos mostrar que para cada elemento da base $B_{s, r} \in{\overline{\left\langle\sigma^{2}\right\rangle_{\mathrm{ab}}}}_{\text {, temos que }}$ $\varepsilon_{n, r_{2}}\left(\mu_{1}\left(B_{s, r}\right)\right)=\overline{0}$, o que é suficiente para que o resultado fique demonstrado.

$1^{o}$ caso: Suponhamos s par. Temos que

$$
\varepsilon_{n, r_{2}}\left(\mu_{1}\left(B_{s, r}\right)\right)=\varepsilon_{n, r_{2}}(0)=\overline{0} .
$$

$2^{\circ}$ caso: Suponhamos $s$ impar e $s \neq 2 n-1$. Temos que

$$
\varepsilon_{n, r_{2}}\left(\mu_{1}\left(B_{s, r}\right)\right)=\varepsilon_{n, r_{2}}\left(B_{s, r}\right)-\varepsilon_{n, r_{2}}\left(B_{s, r-2 r_{2}}\right)=\overline{0}-\overline{0}=\overline{0} .
$$

$3^{o}$ caso: Suponhamos $s=2 n-1$. Notemos que

$$
r+2 r_{2}=r+2(-1)^{j\left(r_{2}\right)} 2^{e\left(r_{2}\right)} o\left(r_{2}\right)=r+2^{e\left(r_{2}\right)+1}(-1)^{j\left(r_{2}\right)} o\left(r_{2}\right) \equiv r \bmod 2^{e\left(r_{2}\right)+1},
$$

e portanto, $\varepsilon_{n, r_{2}}\left(B_{2 n-1, r}\right)=\varepsilon_{n, r_{2}}\left(B_{2 n-1, r-2 r_{2}}\right)$. Assim, temos que

$$
\varepsilon_{n, r_{2}}\left(\mu_{1}\left(B_{2 n-1, r}\right)\right)=\varepsilon_{n, r_{2}}\left(B_{2 n-1, r}-B_{2 n-1, r-2 r_{2}}\right)=\varepsilon_{n, r_{2}}\left(B_{2 n-1, r}\right)-\varepsilon_{n, r_{2}}\left(B_{2 n-1, r-2 r_{2}}\right)=\overline{0} .
$$

Lema 4.4.21. Consideremos o homomorfismo $\nu_{1}:{\overline{\left\langle\sigma^{2}\right\rangle}}_{\mathrm{ab}} \rightarrow{\overline{\left\langle\sigma^{2}\right\rangle}}_{\mathrm{ab}}$ como definido no Lema 4.4.18. Então $\varepsilon_{n, r_{2}} \circ \nu_{1}:{\overline{\left\langle\sigma^{2}\right\rangle}}_{\mathrm{ab}} \rightarrow \mathbb{Z}_{2}$ é identicamente nulo.

Demonstração. Vamos mostrar que para cada elemento da base $B_{s, r} \in{\overline{\left\langle\sigma^{2}\right\rangle_{\mathrm{ab}}}}_{\text {, temos que }}$ $\varepsilon_{n, r_{2}}\left(\nu_{1}\left(B_{s, r}\right)\right)=\overline{0}$, o que é suficiente para que o resultado fique demonstrado.

$1^{o}$ caso: $s$ é par. Notemos que $-s$ e $s+4 n-2$ também são pares e portanto, $-s$ e $s+4 n-2$ são diferentes de $2 n-1$. Logo, temos que

$$
\varepsilon_{n, r_{2}}\left(\nu_{1}\left(B_{s, r}\right)\right)=\varepsilon_{n, r_{2}}\left(B_{-s,-r}\right)-\varepsilon_{n, r_{2}}\left(B_{s+4 n-2, r+2 m-r_{1}}\right)=\overline{0}-\overline{0}=\overline{0} .
$$


$2^{\circ}$ caso: $s$ é ímpar e $s \neq-2 n+1$. Notemos que $-s \neq 2 n-1$ e $s+4 n-2 \neq-2 n+1+4 n-2=2 n-1$. Portanto, $\varepsilon_{n, r_{2}}\left(B_{-s, r-2 m}\right)=\varepsilon_{n, r_{2}}\left(B_{s+4 n-2, r-2 m+r_{1}}\right)=\overline{0}$. Assim, temos que

$$
\varepsilon_{n, r_{2}}\left(\nu_{1}\left(B_{s, r}\right)\right)=-\varepsilon_{n, r_{2}}\left(B_{-s, r-2 m}\right)-\varepsilon_{n, r_{2}}\left(B_{s+4 n-2, r-2 m+r_{1}}\right)=-\overline{0}-\overline{0}=\overline{0} .
$$

$3^{o}$ caso: $s=-2 n+1$. Se $r_{1}=0$, é claro que $r_{1} \equiv 0 \bmod 2^{e\left(r_{2}\right)+1}$. Agora, se $r_{1}>0$ e $e\left(r_{1}\right)>e\left(r_{2}\right)$, então $e\left(r_{1}\right)-e\left(r_{2}\right)-1 \geq 0$. Assim, temos que

$$
r_{1}=2^{e\left(r_{1}\right)} o\left(r_{1}\right)=2^{e\left(r_{2}\right)+1} 2^{e\left(r_{1}\right)-e\left(r_{2}\right)-1} o\left(r_{1}\right) \equiv 0 \bmod 2^{e\left(r_{2}\right)+1} .
$$

Logo, nós podemos concluir que $\varepsilon_{n, r_{2}}\left(B_{2 n-1, r-2 m}\right)=\varepsilon_{n, r_{2}}\left(B_{2 n-1, r-2 m+r_{1}}\right)$. Portanto, temos que

$$
\begin{aligned}
\varepsilon_{n, r_{2}}\left(\nu_{1}\left(B_{-2 n+1, r}\right)\right) & =\varepsilon_{n, r_{2}}\left(-B_{-(-2 n+1), r-2 m}-B_{-2 n+1+4 n-2, r-2 m+r_{1}}\right) \\
& =-\varepsilon_{n, r_{2}}\left(B_{2 n-1, r-2 m}\right)-\varepsilon_{n, r_{2}}\left(B_{2 n-1, r-2 m+r_{1}}\right) \\
& =-2 \varepsilon_{n, r_{2}}\left(B_{2 n-1, r-2 m}\right)=\overline{0} .
\end{aligned}
$$

Lema 4.4.22. Temos que $\varepsilon_{n, r_{2}}\left(\widetilde{J}_{2 n-1,-2 r_{2}}-r_{2} B_{0,0}+r_{2} B_{4 n-2,2 m-r_{1}}\right)=\overline{1}$.

Demonstração. Notemos que para todo $n \in \mathbb{Z}$, temos que $0,4 n-2 \neq 2 n-1$ e portanto, $\varepsilon_{n, r_{2}}\left(-r 2 B_{0,0}+r_{2} B_{4 n-2,2 m-r_{1}}\right)=\overline{0}$. Logo, para que o resultado fique provado, devemos mostrar que $\varepsilon_{n, r_{2}}\left(\widetilde{J}_{2 n-1,-2 r_{2}}\right)=\overline{1}$. Vamos dividir a análise em casos. Utilizaremos a Proposição 4.3 .7 (que nos dá a decomposição de $\widetilde{J}_{2 n-1,-2 r_{2}}$ em termos da base de ${\overline{\left\langle\sigma^{2}\right\rangle}}_{\mathrm{ab}})$ e o Lema 4.4.19.

$1^{o}$ caso: Suponhamos $n \geq 1$. Notemos que se $i$ é um número inteiro, então

$$
2 i-1=2 n-1 \Leftrightarrow i=n .
$$

Notemos ainda que $n \leq 2 n-1$, ou seja, temos que

$$
n \in\{1, \ldots, 2 n-1\} .
$$

Se $r_{2}>0$, seja

$$
S_{1}=-S\left(2^{e\left(r_{2}\right)+1}, o\left(r_{2}\right)\right)=\left\{-x \mid 1 \leq x \leq 2^{e\left(r_{2}\right)+1} o\left(r_{2}\right)=2 r_{2},-x \equiv 0 \bmod 2^{e\left(r_{2}\right)+1}\right\} .
$$

Temos que

$$
\begin{aligned}
\varepsilon_{n, r_{2}}\left(\widetilde{J}_{2 n-1,-2 r_{2}}\right) & =\varepsilon_{n, r_{2}}\left(\sum_{i=1}^{2 n-1} \sum_{j=1}^{2 r_{2}} B_{2 i-1,-j}\right)=\varepsilon_{n, r_{2}}\left(\sum_{\substack{i=1 \\
i \neq n}}^{2 n-1} \sum_{j=1}^{2 r_{2}} B_{2 i-1,-j}\right)+\varepsilon_{n, r_{2}}\left(\sum_{j=1}^{2 r_{2}} B_{2 n-1,-j}\right) \\
& =\overline{0}+\varepsilon_{n, r_{2}}\left(\sum_{j=1}^{2 r_{2}} B_{2 n-1,-j}\right)=\varepsilon_{n, r_{2}}\left(\sum_{\substack{j=-2 r_{2} \\
j \notin S_{1}}}^{-1} B_{2 n-1, j}\right)+\varepsilon_{n, r_{2}}\left(\sum_{j \in S_{1}} B_{2 n-1, j}\right) \\
& =\overline{0}+\varepsilon_{n, r_{2}}\left(\sum_{j \in S_{1}} B_{2 n-1, j}\right)=o\left(r_{2}\right) \overline{1}=\overline{1} .
\end{aligned}
$$

Se $r_{2}<0$, seja

$$
S_{2}=S\left(2^{e\left(r_{2}\right)+1}, o\left(r_{2}\right)\right)-1=\left\{x-1 \mid 1 \leq x \leq 2^{e\left(r_{2}\right)+1} o\left(r_{2}\right)=-2 r_{2}, x-1 \equiv 0 \bmod 2^{e\left(r_{2}\right)+1}\right\} .
$$


Temos que

$$
\begin{aligned}
\varepsilon_{n, r_{2}}\left(\widetilde{J}_{2 n-1,-2 r_{2}}\right) & =\varepsilon_{n, r_{2}}\left(\sum_{i=1}^{2 n-1} \sum_{j=1}^{-2 r_{2}} B_{2 i-1, j-1}\right) \\
& =\varepsilon_{n, r_{2}}\left(\sum_{\substack{i=1 \\
i \neq n}}^{2 n-1} \sum_{j=1}^{-2 r_{2}} B_{2 i-1, j-1}\right)+\varepsilon_{n, r_{2}}\left(\sum_{j=1}^{-2 r_{2}} B_{2 n-1, j-1}\right) \\
& =\overline{0}+\varepsilon_{n, r_{2}}\left(\sum_{j=1}^{-2 r_{2}} B_{2 n-1, j-1}\right)=\varepsilon_{n, r_{2}}\left(\sum_{\substack{j=0 \\
j \notin S_{2}}}^{-2 r_{2}-1} B_{2 n-1, j}\right) \varepsilon_{n, r_{2}}\left(\sum_{j \in S_{2}} B_{2 n-1, j}\right) \\
& =\overline{0}+\varepsilon_{n, r_{2}}\left(\sum_{j \in S_{2}} B_{2 n-1, j}\right)=o\left(r_{2}\right) \overline{1}=\overline{1}
\end{aligned}
$$

$2^{o}$ caso: Suponhamos $n \leq 0$. Notemos que se $i$ é um número inteiro, então

$$
-2 i+1=2 n-1 \Leftrightarrow i=-n+1 .
$$

Notemos ainda que

$$
-n+1 \in\{1, \ldots,-2 n+1\} .
$$

Se $r_{2}>0$, seja novamente

$$
S_{1}=-S\left(2^{e\left(r_{2}\right)+1}, o\left(r_{2}\right)\right)=\left\{-x \mid 1 \leq x \leq 2^{e\left(r_{2}\right)+1} o\left(r_{2}\right)=2 r_{2},-x \equiv 0 \bmod 2^{e\left(r_{2}\right)+1}\right\} .
$$

Temos que

$$
\begin{aligned}
\varepsilon_{n, r_{2}}\left(\widetilde{J}_{2 n-1,-2 r_{2}}\right) & =\varepsilon_{n, r_{2}}\left(-\sum_{i=1}^{-2 n+1} \sum_{j=1}^{2 r_{2}} B_{-2 i+1,-j}\right) \\
& =\varepsilon_{n, r_{2}}\left(\sum_{\substack{i=1 \\
i \neq-n+1}}^{-2 n+1} \sum_{j=1}^{2 r_{2}} B_{-2 i+1,-j}\right)+\varepsilon_{n, r_{2}}\left(\sum_{j=1}^{2 r_{2}} B_{2 n-1,-j}\right) \\
& =\overline{0}+\varepsilon_{n, r_{2}}\left(\sum_{j=1}^{2 r_{2}} B_{2 n-1,-j}\right)=\varepsilon_{n, r_{2}}\left(\sum_{\substack{j=2 r_{2} \\
j \notin S_{1}}}^{-1} B_{2 n-1, j}\right)+\varepsilon_{n, r_{2}}\left(\sum_{j \in S_{1}} B_{2 n-1, j}\right) \\
& =\overline{0}+\varepsilon_{n, r_{2}}\left(\sum_{j \in S_{1}} B_{2 n-1, j}\right)=o\left(r_{2}\right) \overline{1}=\overline{1} .
\end{aligned}
$$

Se $r_{2}<0$, seja novamente

$$
S_{2}=S\left(2^{e\left(r_{2}\right)+1}, o\left(r_{2}\right)\right)-1=\left\{x-1 \mid 1 \leq x \leq 2^{e\left(r_{2}\right)+1} o\left(r_{2}\right)=-2 r_{2}, x-1 \equiv 0 \bmod 2^{e\left(r_{2}\right)+1}\right\} .
$$


Temos que

$$
\begin{aligned}
\varepsilon_{n, r_{2}}\left(\widetilde{J}_{2 n-1,-2 r_{2}}\right) & =\varepsilon_{n, r_{2}}\left(\sum_{i=1}^{-2 n+1} \sum_{j=1}^{-2 r_{2}} B_{-2 i+1, j-1}\right) \\
& =\varepsilon_{n, r_{2}}\left(\sum_{\substack{i=1 \\
i \neq-n+1}}^{-2 n+1} \sum_{j=1}^{-2 r_{2}} B_{-2 i+1, j-1}\right)+\varepsilon_{n, r_{2}}\left(\sum_{j=1}^{-2 r_{2}} B_{2 n-1, j-1}\right) \\
& =\overline{0}+\varepsilon_{n, r_{2}}\left(\sum_{j=1}^{-2 r_{2}} B_{2 n-1, j-1}\right)=\varepsilon_{n, r_{2}}\left(-\sum_{\substack{j=0 \\
j \notin S_{2}}}^{-2 r_{2}-1} B_{2 n-1, j}\right)+\varepsilon_{n, r_{2}}\left(\sum_{j \in S_{2}} B_{2 n-1, j}\right) \\
& =\overline{0}+\varepsilon_{n, r_{2}}\left(\sum_{j \in S_{2}} B_{2 n-1, j}\right)=o\left(r_{2}\right) \overline{1}=\overline{1} .
\end{aligned}
$$

Lema 4.4.23. Não existem elementos $x, y \in{\overline{\left\langle\sigma^{2}\right\rangle}}_{\mathrm{ab}} e(m, n) \in \mathbb{Z} \rtimes \mathbb{Z}$ que satisfazem a equação (1) do Lema 4.4.18.

Demonstração. A prova é por contradição. Suponhamos que existam $x, y \in{\overline{\left\langle\sigma^{2}\right\rangle}}_{\mathrm{ab}}$ e $(m, n) \in \mathbb{Z} \rtimes \mathbb{Z}$ que satisfazem a equação (1) do Lema 4.4.18.

$$
\mu_{1}(x)+\nu_{1}(y)=\widetilde{J}_{2 n-1,-2 r_{2}}-r_{2} B_{0,0}+r_{2} B_{4 n-2,2 m-r_{1}} .
$$

Pelos Lemas 4.4.20, 4.4.21 e 4.4.22, nós temos a seguinte igualdade em $\mathbb{Z}_{2}$

$$
\overline{0}=\varepsilon_{n, r_{2}}\left(\mu_{1}(x)+\nu_{1}(y)\right)=\varepsilon_{n, r_{2}}\left(\widetilde{J}_{2 n-1,-2 r_{2}}-r_{2} B_{0,0}+r_{2} B_{4 n-2,2 m-r_{1}}\right)=\overline{1},
$$

o que é um absurdo. Portanto, não existem tais elementos.

Consideremos agora o homomorfismo $\varepsilon_{n, r_{2}}^{\prime}:{\overline{\left\langle\sigma^{2}\right\rangle}}_{\mathrm{ab}}=\bigoplus_{s, r \in \mathbb{Z}} \mathbb{Z}\left[B_{s, r}\right] \rightarrow \mathbb{Z}_{2}$, o qual é definido em cada elemento da base do seguinte modo:

$$
\varepsilon_{n, r_{2}}^{\prime}\left(B_{s, r}\right)=\left\{\begin{array}{l}
\overline{0}, \text { se } s \neq 2 n \\
\overline{0}, \text { se } s=2 n \text { e } r \not \equiv 0 \bmod 2^{e\left(r_{2}\right)+1} \\
\overline{1}, \text { se } s=2 n \text { e } r \equiv 0 \bmod 2^{e\left(r_{2}\right)+1}
\end{array}\right.
$$

Lema 4.4.24. Consideremos o homomorfismo $\mu_{2}:{\overline{\left\langle\sigma^{2}\right\rangle}}_{\mathrm{ab}} \rightarrow{\overline{\left\langle\sigma^{2}\right\rangle}}_{\mathrm{ab}}$ como definido no Lema 4.4.18. Então $\varepsilon_{n, r_{2}}^{\prime} \circ \mu_{2}:{\overline{\left\langle\sigma^{2}\right\rangle}}_{\mathrm{ab}} \rightarrow \mathbb{Z}_{2}$ é identicamente nulo.

Demonstração. Vamos mostrar que para cada elemento da base $B_{s, r} \in \overline{\left\langle\sigma^{2}\right\rangle}$ ab temos que $\varepsilon_{n, r_{2}}^{\prime}\left(\mu_{2}\left(B_{s, r}\right)\right)=\overline{0}$, o que é suficiente para que o resultado fique demonstrado.

$1^{o}$ caso: Suponhamos s impar. Temos que

$$
\varepsilon_{n, r_{2}}^{\prime}\left(\mu_{2}\left(B_{s, r}\right)\right)=\varepsilon_{n, r_{2}}(0)=\overline{0} .
$$

$2^{\circ}$ caso: Suponhamos $s$ par e $s \neq 2 n$. Temos que

$$
\varepsilon_{n, r_{2}}^{\prime}\left(\mu_{2}\left(B_{s, r}\right)\right)=\varepsilon_{n, r_{2}}^{\prime}\left(B_{s, r+2 r_{2}}\right)-\varepsilon_{n, r_{2}}^{\prime}\left(B_{s, r}\right)=\overline{0}-\overline{0}=\overline{0} .
$$


$3^{\circ}$ caso: Suponhamos $s=2 n$. Notemos que

$$
r+2 r_{2}=r+2(-1)^{j\left(r_{2}\right)} 2^{e\left(r_{2}\right)} o\left(r_{2}\right)=r+2^{e\left(r_{2}\right)+1}(-1)^{j\left(r_{2}\right)} o\left(r_{2}\right) \equiv r \bmod 2^{e\left(r_{2}\right)+1},
$$

e portanto, $\varepsilon_{n, r_{2}}^{\prime}\left(B_{2 n, r}\right)=\varepsilon_{n, r_{2}}^{\prime}\left(B_{2 n, r+2 r_{2}}\right)$. Assim, temos que

$$
\varepsilon_{n, r_{2}}^{\prime}\left(\mu_{1}\left(B_{2 n, r}\right)\right)=\varepsilon_{n, r_{2}}^{\prime}\left(B_{2 n, r+2 r_{2}}-B_{2 n, r}\right)=\varepsilon_{n, r_{2}}^{\prime}\left(B_{2 n, r+2 r_{2}}\right)-\varepsilon_{n, r_{2}}^{\prime}\left(B_{2 n, r}\right)=\overline{0} .
$$

Lema 4.4.25. Consideremos o homomorfismo $\nu_{2}:{\overline{\left\langle\sigma^{2}\right\rangle}}_{\mathrm{ab}} \rightarrow{\overline{\left\langle\sigma^{2}\right\rangle}}_{\mathrm{ab}}$ como definido no Lema 4.4.18. Então $\varepsilon_{n, r_{2}}^{\prime} \circ \nu_{2}:{\overline{\left\langle\sigma^{2}\right\rangle}}_{\mathrm{ab}} \rightarrow \mathbb{Z}_{2}$ é identicamente nulo.

Demonstração. Vamos mostrar que para elemento da base $B_{s, r} \in{\overline{\left\langle\sigma^{2}\right\rangle}}_{\mathrm{ab}}$, temos que $\varepsilon_{n, r_{2}}^{\prime}\left(\nu_{2}\left(B_{s, r}\right)\right)=\overline{0}$, o que é suficiente para que o resultado fique demonstrado.

$1^{o}$ caso: $s$ é ímpar. Notemos que $-s$ e $s+4 n$ também são ímpares e portanto, $-s$ e $s+4 n$ são diferentes de $2 n$. Logo, temos que

$$
\varepsilon_{n, r_{2}}^{\prime}\left(\nu_{2}\left(B_{s, r}\right)\right)=\varepsilon_{n, r_{2}}^{\prime}\left(B_{-s,-r-4 r_{2}-2 m}\right)-\varepsilon_{n, r_{2}}^{\prime}\left(B_{s+4 n, r-r_{1}}\right)=\overline{0}-\overline{0}=\overline{0}
$$

$2^{o}$ caso: $s$ é par e $s \neq-2 n$. Notemos que $-s \neq 2 n$ e $s+4 n \neq-2 n+4 n=2 n$. Portanto, $\varepsilon_{n, r_{2}}^{\prime}\left(B_{-s, r}\right)=\varepsilon_{n, r_{2}}^{\prime}\left(B_{s+4 n, r+r_{1}}\right)=\overline{0}$. Assim, temos que

$$
\varepsilon_{n, r_{2}}^{\prime}\left(\nu_{2}\left(B_{s, r}\right)\right)=-\varepsilon_{n, r_{2}}^{\prime}\left(B_{-s, r}\right)-\varepsilon_{n, r_{2}}^{\prime}\left(B_{s+4 n, r+r_{1}}\right)=-\overline{0}-\overline{0}=\overline{0} .
$$

$3^{o}$ caso: $s=-2 n$. Se $r_{1}=0$, é claro que $r_{1} \equiv 0 \bmod 2^{e\left(r_{2}\right)+1}$. Agora, se $r_{1}>0$ e $e\left(r_{1}\right)>e\left(r_{2}\right)$, então $e\left(r_{1}\right)-e\left(r_{2}\right)-1 \geq 0$. Assim, temos que

$$
r_{1}=2^{e\left(r_{1}\right)} o\left(r_{1}\right)=2^{e\left(r_{2}\right)+1} 2^{e\left(r_{1}\right)-e\left(r_{2}\right)-1} o\left(r_{2}\right) \equiv 0 \bmod 2^{e\left(r_{2}\right)+1} .
$$

Logo, nós podemos concluir que $\varepsilon_{n, r_{2}}^{\prime}\left(B_{2 n, r}\right)=\varepsilon_{n, r_{2}}^{\prime}\left(B_{2 n, r+r_{1}}\right)$. Portanto, temos que

$$
\begin{aligned}
\varepsilon_{n, r_{2}}^{\prime}\left(\nu_{2}\left(B_{-2 n, r}\right)\right) & =\varepsilon_{n, r_{2}}^{\prime}\left(-B_{-(-2 n), r}-B_{-2 n+4 n, r+r_{1}}\right) \\
& =-\varepsilon_{n, r_{2}}^{\prime}\left(B_{2 n, r}\right)-\varepsilon_{n, r_{2}}^{\prime}\left(B_{2 n, r+r_{1}}\right)=-2 \varepsilon_{n, r_{2}}^{\prime}\left(B_{2 n, r}\right)=\overline{0} .
\end{aligned}
$$

Lema 4.4.26. Consideremos o elemento $\widetilde{J}_{2 n,-2 r_{2}} \in{\overline{\left\langle\sigma^{2}\right\rangle}}_{\mathrm{ab}}$. Então $\varepsilon_{n, r_{2}}^{\prime}\left(\widetilde{J}_{2 n,-2 r_{2}}\right)=\overline{0}$.

Demonstração. Se $n=0$, então pela Proposição 4.3.7, temos que $\widetilde{J}_{0,-2 r_{2}}=0$ e portanto $\varepsilon_{0, r_{2}}^{\prime}\left(\widetilde{J}_{0,-2 r_{2}}\right)=$ $\overline{0}$. Se $n \neq 0$, a prova segue diretamente da Proposição 4.3 .7 e notando que $\varepsilon_{n, r_{2}}^{\prime}\left(B_{s, r}\right)=\overline{0}$ se $s$ é impar.

Lema 4.4.27. Consideremos o elemento $\widetilde{O}_{2 n, 2 r_{2}} \in{\overline{\left\langle\sigma^{2}\right\rangle}}_{\mathrm{ab}}$. Então $\varepsilon_{n, r_{2}}^{\prime}\left(\widetilde{O}_{2 n, 2 r_{2}}\right)=\left\{\begin{array}{l}\overline{0}, \text { se } n=0 \\ \overline{1}, \text { se } n \neq 0 .\end{array}\right.$

Demonstração. Pela Proposição 4.3.7, se $n=0$, então $\widetilde{O}_{0,2 r_{2}}=0$, e portanto $\varepsilon_{0, r_{2}}^{\prime}\left(\widetilde{O}_{0,2 r_{2}}\right)=\overline{0}$. Suponhamos então $n \neq 0$. Vamos dividir a análise em casos. Utilizaremos a Proposição 4.3 .7 (que nos dá a decomposição de $\widetilde{O}_{2 n, 2 r_{2}}$ em termos da base de ${\overline{\left\langle\sigma^{2}\right\rangle}}_{\mathrm{ab}}$ ) e o Lema 4.4.19. 
$1^{o}$ caso: Suponhamos $n \geq 1$. Notemos que se $i$ é um número inteiro, então

$$
2 i-2=2 n \Leftrightarrow i=n+1
$$

Notemos ainda que $n+1 \leq 2 n$, ou seja, temos que

$$
n+1 \in\{1, \ldots, 2 n\} .
$$

Lembremos que $\varepsilon_{n, r_{2}}^{\prime}\left(B_{s, r}\right)=\overline{0}$ se $s$ é ímpar.

Se $r_{2}>0$, seja

$$
S_{1}=S\left(2^{e\left(r_{2}\right)+1}, o\left(r_{2}\right)\right)-1=\left\{x-1 \mid 1 \leq x \leq 2^{e\left(r_{2}\right)+1} o\left(r_{2}\right)=2 r_{2}, x-1 \equiv 0 \bmod 2^{e\left(r_{2}\right)+1}\right\} .
$$

Temos que

$$
\begin{aligned}
\varepsilon_{n, r_{2}}^{\prime}\left(\widetilde{O}_{2 n, 2 r_{2}}\right) & =\varepsilon_{n, r_{2}}^{\prime}\left(\sum_{i=1}^{2 n} \sum_{j=1}^{2 r_{2}}\left(B_{2 i-1,-j}-B_{2 i-2, j-1}\right)\right) \\
& =\varepsilon_{n, r_{2}}^{\prime}\left(\sum_{i=1}^{2 n} \sum_{j=1}^{2 r_{2}} B_{2 i-1,-j}\right)+\varepsilon_{n, r_{2}}^{\prime}\left(\sum_{\substack{i=1 \\
i \neq n+1}}^{2 n} \sum_{j=1}^{2 r_{2}} B_{2 i-2, j-1}\right)+\varepsilon_{n, r_{2}}^{\prime}\left(\sum_{j=1}^{2 r_{2}} B_{2 n, j-1}\right) \\
& =\overline{0}+\overline{0}+\varepsilon_{n, r_{2}}^{\prime}\left(\sum_{j=1}^{2 r_{2}} B_{2 n, j-1}\right)=\varepsilon_{n, r_{2}}^{\prime}\left(\sum_{\substack{j=0 \\
j \notin S_{1}}}^{2 r_{2}-1} B_{2 n, j}\right)+\varepsilon_{n, r_{2}}^{\prime}\left(\sum_{j \in S_{1}} B_{2 n, j}\right) \\
& =\overline{0}+\varepsilon_{n, r_{2}}^{\prime}\left(\sum_{j \in S_{1}} B_{2 n, j}\right)=o\left(r_{2}\right) \overline{1}=\overline{1} .
\end{aligned}
$$

Se $r_{2}<0$, seja

$$
S_{2}=-S\left(2^{e\left(r_{2}\right)+1}, o\left(r_{2}\right)\right)=\left\{-x \mid 1 \leq x \leq 2^{e\left(r_{2}\right)+1} o\left(r_{2}\right)=-2 r_{2},-x \equiv 0 \bmod 2^{e\left(r_{2}\right)+1}\right\} .
$$

Temos que

$$
\begin{aligned}
\varepsilon_{n, r_{2}}^{\prime}\left(\widetilde{O}_{2 n, 2 r_{2}}\right) & =\varepsilon_{n, r_{2}}^{\prime}\left(\sum_{i=1}^{2 n} \sum_{j=1}^{-2 r_{2}}\left(B_{2 i-2,-j}-B_{2 i-1, j-1}\right)\right) \\
& =\varepsilon_{n, r_{2}}^{\prime}\left(\sum_{\substack{i=1 \\
i \neq n+1}}^{2 n} \sum_{j=1}^{-2 r_{2}}\left(B_{2 i-2,-j}\right)\right)+\varepsilon_{n, r_{2}}^{\prime}\left(\sum_{j=1}^{-2 r_{2}} B_{2 n,-j}\right)+\varepsilon_{n, r_{2}}^{\prime}\left(\sum_{i=1}^{2 n} \sum_{j=1}^{-2 r_{2}}\left(B_{2 i-1, j-1}\right)\right) \\
& =\overline{0}+\varepsilon_{n, r_{2}}^{\prime}\left(\sum_{j=1}^{-2 r_{2}} B_{2 n,-j}\right)+\overline{0}=\varepsilon_{n, r_{2}}^{\prime}\left(\sum_{j=2 r_{2}}^{-1} B_{2 n, j}\right)+\varepsilon_{n, r_{2}}^{\prime}\left(\sum_{j \in S_{2}} B_{2 n, j}\right) \\
& =\overline{0}+\varepsilon_{n, r_{2}}^{\prime}\left(\sum_{j \in S_{2}} B_{2 n, j}\right)=o\left(r_{2}\right) \overline{1}=\overline{1} .
\end{aligned}
$$

$2^{o}$ caso: Suponhamos $n \leq-1$. Notemos que se $i$ é um número inteiro, então

$$
-2 i=2 n \Leftrightarrow i=-n \text {. }
$$


Notemos ainda que $-n \leq-2 n$, ou seja, temos que

$$
-n \in\{1, \ldots,-2 n\} .
$$

Lembremos que $\varepsilon_{n, r_{2}}^{\prime}\left(B_{s, r}\right)=\overline{0}$ se $s$ é ímpar.

Se $r_{2}>0$, seja novamente

$$
S_{1}=S\left(2^{e\left(r_{2}\right)+1}, o\left(r_{2}\right)\right)-1=\left\{x-1 \mid 1 \leq x \leq 2^{e\left(r_{2}\right)+1} o\left(r_{2}\right)=2 r_{2}, x-1 \equiv 0 \bmod 2^{e\left(r_{2}\right)+1}\right\} .
$$

Temos que

$$
\begin{aligned}
\varepsilon_{n, r_{2}}^{\prime}\left(\widetilde{O}_{2 n, 2 r_{2}}\right) & =\varepsilon_{n, r_{2}}^{\prime}\left(\sum_{i=1}^{-2 n} \sum_{j=1}^{2 r_{2}}\left(B_{-2 i, j-1}-B_{-2 i+1,-j}\right)\right) \\
& =\varepsilon_{n, r_{2}}^{\prime}\left(\sum_{\substack{i=1 \\
i \neq-n}}^{-2 n} \sum_{j=1}^{2 r_{2}} B_{-2 i, j-1}\right)+\varepsilon_{n, r_{2}}^{\prime}\left(\sum_{j=1}^{2 r_{2}} B_{2 n, j-1}\right)+\varepsilon_{n, r_{2}}^{\prime}\left(\sum_{i=1}^{-2 n} \sum_{j=1}^{2 r_{2}}\left(B_{-2 i+1,-j}\right)\right) \\
& =\overline{0}+\varepsilon_{n, r_{2}}^{\prime}\left(\sum_{j=1}^{2 r_{2}} B_{2 n, j-1}\right)+\overline{0}=\varepsilon_{n, r_{2}}^{\prime}\left(\sum_{\substack{j=0 \\
j \notin S_{1}}}^{2 r_{2}-1} B_{2 n, j}\right)+\varepsilon_{n, r_{2}}^{\prime}\left(\sum_{j \in S_{1}} B_{2 n, j}\right) \\
& =\overline{0}+\varepsilon_{n, r_{2}}^{\prime}\left(\sum_{j \in S_{1}} B_{2 n, j}\right)=o\left(r_{2}\right) \overline{1}=\overline{1} .
\end{aligned}
$$

Se $r_{2}<0$, seja novamente

$$
S_{2}=-S\left(2^{e\left(r_{2}\right)+1}, o\left(r_{2}\right)\right)=\left\{-x \mid 1 \leq x \leq 2^{e\left(r_{2}\right)+1} o\left(r_{2}\right)=-2 r_{2},-x \equiv 0 \bmod 2^{e\left(r_{2}\right)+1}\right\} .
$$

Temos que

$$
\begin{aligned}
\varepsilon_{n, r_{2}}^{\prime}\left(\widetilde{O}_{2 n, 2 r_{2}}\right) & =\varepsilon_{n, r_{2}}^{\prime}\left(\sum_{i=1}^{-2 n} \sum_{j=1}^{-2 r_{2}}\left(B_{-2 i+1, j-1}-B_{-2 i,-j}\right)\right) \\
& =\varepsilon_{n, r_{2}}^{\prime}\left(\sum_{i=1}^{-2 n} \sum_{j=1}^{-2 r_{2}} B_{-2 i+1, j-1}\right)+\varepsilon_{n, r_{2}}^{\prime}\left(\sum_{\substack{i=1 \\
i \neq-n}}^{-2 n} \sum_{j=1}^{-2 r_{2}} B_{-2 i,-j}\right)+\varepsilon_{n, r_{2}}^{\prime}\left(\sum_{j=1}^{-2 r_{2}} B_{2 n,-j}\right) \\
& =\overline{0}+\overline{0}+\varepsilon_{n, r_{2}}^{\prime}\left(\sum_{j=1}^{-2 r_{2}} B_{2 n,-j}\right)=\varepsilon_{n, r_{2}}^{\prime}\left(\sum_{\substack{j=2 r_{2} \\
j \notin S_{2}}}^{-1} B_{2 n, j}\right)+\varepsilon_{n, r_{2}}^{\prime}\left(\sum_{j \in S_{2}} B_{2 n, j}\right) \\
& =\overline{0}+\varepsilon_{n, r_{2}}^{\prime}\left(\sum_{j \in S_{2}} B_{2 n, j}\right)=o\left(r_{2}\right) \overline{1}=\overline{1} .
\end{aligned}
$$

Lema 4.4.28. Consideremos o elemento $\widetilde{W}_{2 r_{2}} \in{\overline{\left\langle\sigma^{2}\right\rangle}}_{\mathrm{ab}}$. Então $\varepsilon_{n, r_{2}}^{\prime}\left(\widetilde{W}_{2 r_{2}}\right)=\left\{\begin{array}{l}\overline{1}, \text { se } n=0 ; \\ \overline{0}, \text { se } n \neq 0 .\end{array}\right.$

Demonstração. Notemos que se $n \neq 0$, então $\varepsilon_{n, r_{2}}^{\prime}\left(B_{0, r}\right)=\overline{0}$. Assim, segue diretamente da Proposição 4.3.7 que $\varepsilon_{n, r_{2}}^{\prime}\left(\widetilde{W}_{2 r_{2}}\right)=\overline{0}$ se $n \neq 0$. Resta mostrar que $\varepsilon_{0, r_{2}}^{\prime}\left(\widetilde{W}_{2 r_{2}}\right)=\overline{1}$. Vamos usar a Proposição 4.3.7 (que nos dá a decomposição de $\widetilde{W}_{2 r_{2}}$ em termos da base de ${\overline{\left\langle\sigma^{2}\right\rangle}}_{\mathrm{ab}}$ ) e o Lema 4.4.19. 
Se $r_{2}>0$, seja

$$
S_{1}=S\left(2^{e\left(r_{2}\right)+1}, o\left(r_{2}\right)\right)-1=\left\{x-1 \mid 1 \leq x \leq 2^{e\left(r_{2}\right)+1} o\left(r_{2}\right)=2 r_{2}, x-1 \equiv 0 \bmod 2^{e\left(r_{2}\right)+1}\right\} .
$$

Temos que

$$
\begin{aligned}
\varepsilon_{0, r_{2}}^{\prime}\left(\widetilde{W}_{2 r_{2}}\right) & =\varepsilon_{0, r_{2}}^{\prime}\left(\sum_{j=1}^{2 r_{2}} B_{0, j-1}\right)=\varepsilon_{0, r_{2}}^{\prime}\left(\sum_{\substack{j=0 \\
j \notin S_{1}}}^{2 r_{2}-1} B_{0, j}\right)+\varepsilon_{0, r_{2}}^{\prime}\left(\sum_{j \in S_{1}} B_{0, j}\right) \\
& =\overline{0}+\varepsilon_{0, r_{2}}^{\prime}\left(\sum_{j \in S_{1}} B_{0, j}\right)=o\left(r_{2}\right) \overline{1}=\overline{1} .
\end{aligned}
$$

Se $r_{2}<0$, seja

$$
S_{2}=-S\left(2^{e\left(r_{2}\right)+1}, o\left(r_{2}\right)\right)=\left\{-x \mid 1 \leq x \leq 2^{e\left(r_{2}\right)+1} o\left(r_{2}\right)=-2 r_{2},-x \equiv 0 \bmod 2^{e\left(r_{2}\right)+1}\right\} .
$$

Temos que

$$
\begin{aligned}
\varepsilon_{0, r_{2}}^{\prime}\left(\widetilde{W}_{2 r_{2}}\right) & =\varepsilon_{0, r_{2}}^{\prime}\left(-\sum_{j=1}^{-2 r_{2}} B_{0,-j}\right)=\varepsilon_{0, r_{2}}^{\prime}\left(\sum_{\substack{j=2 r_{2} \\
j \notin S_{2}}}^{-1} B_{0, j}\right) \varepsilon_{0, r_{2}}^{\prime}\left(\sum_{j \in S_{2}} B_{0, j}\right) \\
& =\overline{0}+\varepsilon_{0, r_{2}}^{\prime}\left(\sum_{j \in S_{2}} B_{0, j}\right)=o\left(r_{2}\right) \overline{1}=\overline{1} .
\end{aligned}
$$

Lema 4.4.29. Consideremos o elemento $\left(r_{2}+m-1\right) B_{0,0}+(1-m) B_{0,2 r_{2}}+r_{2} B_{4 n, r_{1}} \in{\overline{\left\langle\sigma^{2}\right\rangle}}_{\mathrm{ab}}$. Então $\varepsilon_{n, r_{2}}^{\prime}\left(\left(r_{2}+m-1\right) B_{0,0}+(1-m) B_{0,2 r_{2}}+r_{2} B_{4 n, r_{1}}\right)=\overline{0}$.

Demonstração. Se $n \neq 0$, então $2 n \neq 4 n$. Assim, temos que

$$
\varepsilon_{n, r_{2}}^{\prime}\left(\left(r_{2}+m-1\right) B_{0,0}+(1-m) B_{0,2 r_{2}}+r_{2} B_{4 n, r_{1}}\right)=\left(r_{2}+m-1\right) \overline{0}+(1-m) \overline{0}+r_{2} \overline{0}=\overline{0} .
$$

Suponhamos então $n=0$. Notemos que

$$
2 r_{2}=2(-1)^{j\left(r_{2}\right)} 2^{e\left(r_{2}\right)} o\left(r_{2}\right)=2^{e\left(r_{2}\right)+1}(-1)^{j\left(r_{2}\right)} o\left(r_{2}\right) \equiv 0 \bmod 2^{e\left(r_{2}\right)+1} .
$$

Logo, $\varepsilon_{0, r_{2}}^{\prime}\left(B_{0,2 r_{2}}\right)=\overline{1}$.

Se $r_{1}=0$, é claro que $r_{1} \equiv 0 \bmod 2^{e\left(r_{2}\right)+1}$. Se $r_{1} \neq 0$ e $e\left(r_{1}\right)>e\left(r_{2}\right)$, então $e\left(r_{1}\right)-e\left(r_{2}\right)-1 \geq 0$. Assim, temos que

$$
r_{1}=2^{e\left(r_{1}\right)} o\left(r_{1}\right)=2^{e\left(r_{2}\right)+1} 2^{e\left(r_{1}\right)-e\left(r_{2}\right)-1} o\left(r_{1}\right) \equiv 0 \bmod 2^{e\left(r_{2}\right)+1} .
$$

Logo, $\varepsilon_{0, r_{2}}^{\prime}\left(B_{0, r_{1}}\right)=\overline{1}$.

Assim, temos que

$$
\varepsilon_{0, r_{2}}^{\prime}\left(\left(r_{2}+m-1\right) B_{0,0}+(1-m) B_{0,2 r_{2}}+r_{2} B_{0, r_{1}}\right)=\left(r_{2}+m-1\right) \overline{1}+(1-m) \overline{1}+r_{2} \overline{1}=2 r_{2} \overline{1}=\overline{0} .
$$


Lema 4.4.30. Nâo existem elementos $x, y \in{\overline{\left\langle\sigma^{2}\right\rangle}}_{\mathrm{ab}}$ e $(m, n) \in \mathbb{Z} \rtimes \mathbb{Z}$ que satisfazem a equação (2) do Lema 4.4.18.

Demonstração. A prova é por contradição. Suponhamos que existam $x, y \in{\overline{\left\langle\sigma^{2}\right\rangle}}_{\mathrm{ab}}$ e $(m, n) \in \mathbb{Z} \rtimes \mathbb{Z}$ que satisfazem a equação

$$
\mu_{2}(x)+\nu_{2}(y)=\widetilde{J}_{2 n,-2 r_{2}}-\widetilde{O}_{2 n, 2 r_{2}}-\widetilde{W}_{2 r_{2}}+\left(r_{2}+m+1\right) B_{0,0}+(1-m) B_{0,2 r_{2}}+r_{2} B_{4 n, r_{1}} .
$$

Pelos Lemas 4.4.24, 4.4.25, 4.4.26, 4.4.27, 4.4.28 e 4.4.29, nós temos a seguinte igualdade em $\mathbb{Z}_{2}$

$$
\begin{aligned}
\overline{0}=\varepsilon_{n, r_{2}}^{\prime} & \left(\mu_{2}(x)+\nu_{2}(y)\right) \\
& =\varepsilon_{n, r_{2}}^{\prime}\left(\widetilde{J}_{2 n,-2 r_{2}}-\widetilde{O}_{2 n, 2 r_{2}}-\widetilde{W}_{2 r_{2}}+\left(r_{2}+m-1\right) B_{0,0}+(1-m) B_{0,2 r_{2}}+r_{2} B_{4 n, r_{1}}\right)=\overline{1},
\end{aligned}
$$

o que é um absurdo. Portanto, não existem tais elementos.

Demonstração das Proposiçôes 4.4.8 e 4.4.10. Queremos mostrar que se $\alpha \in\left[\mathbb{T}^{2}, * ; \mathbb{K}^{2}, *\right]$ é uma classe de homotopia pontuada cujo homomorfismo $h_{\alpha}=\Gamma(\alpha): \pi_{1}\left(\mathbb{T}^{2}\right) \rightarrow \pi_{1}\left(\mathbb{K}^{2}\right)$ é tal que

$$
h_{\alpha}(1,0)=\left(r_{1}, 2 s_{1}\right) \quad \text { e } \quad h_{\alpha}(0,1)=\left(r_{2}, 2 s_{2}\right),
$$

para algum $r_{1}, s_{1}, r_{2}, s_{2} \in \mathbb{Z}$, sendo $s_{1}$ ímpar, $r_{2} \neq 0, r_{1}=0$ ou $r_{1}>0$ e $e\left(r_{1}\right)>e\left(r_{2}\right)$, então $\alpha$ tem a propriedade de Borsuk-Ulam. Pelo Corolário 4.4.12, como $2 s_{1} \equiv 2 \bmod 4$, para que este resultado fique provado basta mostrarmos que se $\alpha \in\left[\mathbb{T}^{2}, * ; \mathbb{K}^{2}, *\right]$ é uma classe de homotopia pontuada cujo homomorfismo $h_{\alpha}=\Gamma(\alpha): \pi_{1}\left(\mathbb{T}^{2}\right) \rightarrow \pi_{1}\left(\mathbb{K}^{2}\right)$ é tal que

$$
h_{\alpha}(1,0)=\left(r_{1}, 2\right) \quad \text { e } \quad h_{\alpha}(0,1)=\left(r_{2}, 0\right),
$$

para algum $r_{1}, r_{2} \in \mathbb{Z}$, sendo $r_{2} \neq 0, r_{1}=0$ ou $r_{1}>0$ e $e\left(r_{1}\right)>e\left(r_{2}\right)$, então $\alpha$ tem a propriedade de Borsuk-Ulam. Mas este fato segue diretamente dos Lemas 4.4.18, 4.4.23 e 4.4.30. 


\section{Capítulo 5}

\section{Os $\operatorname{casos}\left(M, \tau ; \mathbb{S}^{2}\right)$ e $\left(M, \tau ; \mathbb{R P}^{2}\right)$}

\subsection{Introdução}

Neste capítulo nós vamos estudar a propriedade de Borsuk-Ulam para classes de homotopia de funções que têm como domínio uma superfície fechada e como contra-domínio a esfera 2-dimensional ou o plano projetivo. Mais precisamente, dada uma superfície fechada $M$ e uma involução livre de pontos fixos $\tau: M \rightarrow M$, nós vamos classificar todas as classes de homotopia $\beta \in\left[M, \mathbb{S}^{2}\right]$ que têm a propriedade de Borsuk-Ulam (Teoremas 5.1.2, 5.1.3 e 5.1.4). Também vamos classificar as classes de homotopia $\alpha \in\left[M, \mathbb{R P}^{2}\right]$ que se levantam para a $\mathbb{S}^{2}$ em relação a propriedade de Borsuk-Ulam (Teoremas 5.1.5 e 5.1.6). Antes de enunciar os resultados de classificação, primeiramente nós vamos enunciar alguns fatos e fixar algumas notações. Nas Seções 5.2 e 5.3 faremos as demonstrações.

Lembremos da Seção 1.3, que sempre que temos uma involução livre de pontos fixos $\tau: M \rightarrow M$, definimos a seguinte relação de equivalência em $M: x \sim y \Leftrightarrow y \in\{x, \tau(x)\}$. Denotamos por $M_{\tau}$ o conjunto das classes de equivalência, também chamado de espaço de órbitas, e por $p_{\tau}: M \rightarrow M_{\tau}$ a aplicação quociente. Se $M$ é uma superfície fechada, então $M_{\tau}$ também o é, e assim $p_{\tau}$ é um recobrimento duplo. Assim, temos a seguinte sequência exata (omitimos os pontos bases):

$$
1 \longrightarrow \pi_{1}(M) \stackrel{\left(p_{\tau}\right)_{\#}}{\longrightarrow} \pi_{1}\left(M_{\tau}\right) \stackrel{\theta_{\tau}}{\longrightarrow} \frac{\pi_{1}\left(M_{\tau}\right)}{\left(p_{\tau}\right)_{\#}\left(\pi_{1}(M)\right)} \cong \mathbb{Z}_{2} \longrightarrow .
$$

No caso em que $M_{\tau}$ é não orientável, dependendo da característica de Euler de $M_{\tau}$, nós temos as seguintes possibilidades para presentação de $\pi_{1}\left(M_{\tau}\right)$ :

- $\pi_{1}\left(M_{\tau}\right) \cong\left\langle u, v, a_{1}, a_{2}, \cdots, a_{2 n-1}, a_{2 n} \mid u v u v^{-1}\left[a_{1}, a_{2}\right] \ldots\left[a_{2 n-1}, a_{2 n}\right]=1\right\rangle$, se $\chi\left(M_{\tau}\right)$ é par;

- $\pi_{1}\left(M_{\tau}\right) \cong\left\langle c, a_{1}, a_{2}, \cdots, a_{2 n-1}, a_{2 n} \mid c^{2}\left[a_{1}, a_{2}\right] \ldots\left[a_{2 n-1}, a_{2 n}\right]=1\right\rangle$, se $\chi\left(M_{\tau}\right)$ é ímpar.

Para que possamos encurtar certos enunciados e demonstrações, nós destacamos o seguinte elemento de $\pi_{1}\left(M_{\tau}\right)$ :

$$
\delta=\left\{\begin{array}{l}
u, \text { se } \chi\left(M_{\tau}\right) \text { é par; } \\
c, \text { se } \chi\left(M_{\tau}\right) \text { é ímpar. }
\end{array}\right.
$$

Sobre o conjunto $\left[M, \mathbb{S}^{2}\right]$, é bem conhecido o fato que duas funções são homotópicas se, e somente se, elas têm o mesmo grau. Tal fato está descrito, por exemplo, em [27, Capítulo V, Teorema 6.17]. Vamos descrever mais precisamente este resultado. 
Suponhamos que $M$ seja uma superfície fechada orientável. Lembremos que $\mathrm{H}^{2}(M ; \mathbb{Z})$ é cíclico infinito. Seja $z_{m}$ um gerador fixado. Fixemos também um gerador $z_{s}$ do grupo cíclico infinito $\mathrm{H}^{2}\left(\mathbb{S}^{2} ; \mathbb{Z}\right)$. Se $f: M \rightarrow \mathbb{S}^{2}$ é uma função, então existe um único número inteiro $d(f)$, chamado grau de $f$, tal que

$$
\begin{aligned}
f^{*}: \mathrm{H}^{2}\left(\mathbb{S}^{2} ; \mathbb{Z}\right) & \longrightarrow \mathrm{H}^{2}(M ; \mathbb{Z}) \\
z_{s} & \longmapsto d(f) z_{m} .
\end{aligned}
$$

Suponhamos agora que $M$ seja uma superfície fechada não orientável. Então $\mathrm{H}^{2}\left(M ; \mathbb{Z}_{2}\right)$ é cíclico de ordem 2 , assim como $\mathrm{H}^{2}\left(\mathbb{S}^{2} ; \mathbb{Z}_{2}\right)$. Sejam $\bar{z}_{m}$ e $\bar{z}_{s}$ os respectivos geradores. Se $f: M \rightarrow \mathbb{S}^{2}$ é uma função, então existe um único elemento $d(f, 2) \in \mathbb{Z}_{2}$, chamado grau módulo 2 de $f$, tal que

$$
\begin{aligned}
f^{*}: \mathrm{H}^{2}\left(\mathbb{S}^{2} ; \mathbb{Z}_{2}\right) & \longrightarrow \mathrm{H}^{2}\left(M ; \mathbb{Z}_{2}\right) \\
\bar{z}_{s} & \longmapsto d(f, 2) \bar{z}_{m} .
\end{aligned}
$$

Vale o seguinte resultado:

Teorema 5.1.1. As seguintes funçôes são bem definidas e bijeçôes:

$$
\begin{aligned}
& \operatorname{deg}:\left[M, \mathbb{S}^{2}\right] \longrightarrow \mathbb{Z} \\
& \beta=[f] \longmapsto \operatorname{deg}(\beta)=d(f) \\
& \text { se } M \text { é orientável; }
\end{aligned}
$$$$
\begin{aligned}
\overline{\operatorname{deg}:}\left[M, \mathbb{S}^{2}\right] & \longrightarrow \mathbb{Z}_{2} \\
\beta=[f] & \longmapsto \overline{\operatorname{deg}}(\beta)=d(f, 2)
\end{aligned}
$$$$
\text { se } M \text { é não orientável. }
$$

Denotemos por $A: \mathbb{S}^{2} \rightarrow \mathbb{S}^{2}$ a involução antipodal. Lembremos que, por definição, $\mathbb{R P}^{2}=\mathbb{S}_{A}^{2}$.

Fixemos $g: M \rightarrow \mathbb{R P}^{2}$ uma função. Suponhamos que $g_{\#}: \pi_{1}\left(M, m_{1}\right) \rightarrow \pi_{1}\left(\mathbb{R P}^{2}, g\left(m_{1}\right)\right)$ é trivial. Lembremos da teoria de grupo fundamental que se trocarmos $m_{1}$ por outro ponto base $m_{1}^{\prime}$, então $g_{\#}: \pi_{1}\left(M, m_{1}^{\prime}\right) \rightarrow \pi_{1}\left(\mathbb{R P}^{2}, g\left(m_{1}^{\prime}\right)\right)$ também é trivial. Portanto, a propriedade de $g_{\#}$ ser trivial pode ser enunciada omitindo os pontos base.

Com estes fatos e notações fixadas, o objetivo deste capítulo é mostrar os seguintes resultados de classificação:

Teorema 5.1.2. Sejam $M$ uma superfície fechada orientável e $\tau: M \rightarrow M$ uma involução livre de pontos fixos tal que o espaço de órbitas $M_{\tau}$ é orientável. Uma classe de homotopia $\beta \in\left[M, \mathbb{S}^{2}\right]$ tem a propriedade de Borsuk-Ulam se, e somente se, $\beta$ não é a classe de homotopia de uma função constante.

Teorema 5.1.3. Sejam $M$ uma superfície fechada orientável e $\tau: M \rightarrow M$ uma involução livre de pontos fixos tal que o espaço de órbitas $M_{\tau}$ é não orientável. Uma classe de homotopia $\beta \in\left[M, \mathbb{S}^{2}\right]$ tem a propriedade de Borsuk-Ulam se, e somente se, $\chi\left(M_{\tau}\right)+\operatorname{deg}(\beta)$ é impar, sendo $\operatorname{deg}:\left[M, \mathbb{S}^{2}\right] \rightarrow \mathbb{Z}$ como definido no Teorema 5.1.1.

Teorema 5.1.4. Sejam $M$ uma superfície fechada não orientável e $\tau: M \rightarrow M$ uma involução livre de pontos fixos (como $p_{\tau}: M \rightarrow M_{\tau}$ é um recobrimento, então $M_{\tau}$ é não orientável). Existe apenas uma classe de homotopia $\beta \in\left[M, \mathbb{S}^{2}\right]$ em que vale a propriedade de Borsuk-Ulam. Mais ainda, são verdadeiras as seguintes afirmaçôes, sendo $\theta_{\tau}: \pi_{1}\left(M_{\tau}\right) \rightarrow \mathbb{Z}_{2}$ como definido em (5.1) e $\delta$ como definido em (5.2):

1. Se $\theta_{\tau}(\delta)=\overline{0}$, então $\beta$ não é a classe de homotopia de uma função constante;

2. Se $\theta_{\tau}(\delta)=\overline{1}$, então $\beta$ é a classe de homotopia de uma função constante.

Teorema 5.1.5. Sejam $M$ uma superfície fechada orientável, $\tau: M \rightarrow M$ uma involução livre de pontos fixos e $\alpha \in\left[M, \mathbb{R P}^{2}\right]$ uma classe de homotopia tal que $g_{\#}: \pi_{1}(M) \rightarrow \pi_{1}\left(\mathbb{R P}^{2}\right)$ é trivial, sendo $g: M \rightarrow \mathbb{R P}^{2}$ um representante de $\alpha$. Então a tem a propriedade de Borsuk-Ulam se, $e$ somente se, uma das seguintes condições é satisfeita: 
1. $\alpha$ não é a classe de homotopia de uma função constante;

2. o espaço de órbitas $M_{\tau}$ é não orientável e $\chi\left(M_{\tau}\right)$ é ímpar.

Teorema 5.1.6. Sejam $M$ uma superfície fechada não orientável, $\tau: M \rightarrow M$ uma involução livre de pontos fixos e $\alpha \in\left[M, \mathbb{R P}^{2}\right]$ uma classe de homotopia tal que $g_{\#}: \pi_{1}(M) \rightarrow \pi_{1}\left(\mathbb{R P}^{2}\right)$ é trivial, sendo $g: M \rightarrow \mathbb{R P}^{2}$ um representante de $\alpha$. Valem as seguintes afirmaçôes:

1. se $\theta_{\tau}(\delta)=\overline{0}$, então $\alpha$ tem a propriedade de Borsuk-Ulam se, e somente se, $\alpha$ não é a classe de homotopia de uma função constante.

2. se $\theta_{\tau}(\delta)=\overline{1}$, então a tem a propriedade de Borsuk-Ulam.

\subsection{Os $\operatorname{casos}\left(M, \tau ; \mathbb{S}^{2}\right)$}

O objetivo desta seção é estudar a propriedade de Borsuk-Ulam para funções que têm como contra-domínio $\mathbb{S}^{2}$ e demonstrar os Teoremas 5.1.2, 5.1.3 e 5.1.4. Notemos que estes casos não satisfazem as hipóteses do Lema Fundamental 1.4.3. Entretanto, usando a geometria de $\mathbb{S}^{2}$, nós mostraremos que estudar a propriedade de Borsuk-Ulam para classes de homotopia é, no sentido do Lema 5.2.1 que iremos demonstrar, dual ao problema de estudar quais classes de homotopia têm representantes equivariantes. Este resultado será fundamental nas demonstrações dos resultados de classificação.

Ainda nesta seção, nós enunciaremos um resultado sobre grau módulo 2 (Proposição 5.2.2), o qual usaremos para demonstrar o Lema 5.2.3. Este resultado nos será útil quando estivermos estudando o problema de Borsuk-Ulam para funções cujo domínio esteja munido de uma involução tal que o espaço de órbitas é não orientável.

O objetivo das Subseções 5.2.1, 5.2.2 e 5.2.3 é de fato provar os Teoremas 5.1.2, 5.1.3 e 5.1.4, respectivamente. Na Subseção 5.2.2 mostraremos alguns resultados preliminares.

Lema 5.2.1. Sejam $M$ uma superfície fechada, $\tau: M \rightarrow M$ uma involução livre de pontos fixos e $\beta \in\left[M, \mathbb{S}^{2}\right]$ uma classe de homotopia. As seguintes condiçôes são equivalentes:

1. $\beta$ não tem a propriedade de Borsuk-Ulam com respeito a $\tau$;

2. $\beta$ tem um representante $f: M \rightarrow \mathbb{S}^{2}$ que é $(\tau, A)$-equivariante, isto é, $\beta=[f]$ e para cada $x \in M$ vale $f(\tau(x))=A(f(x))$.

Demonstração. A prova de que a condição 2 implica a condição 1 é igual ao final da demonstração do Lema 1.4.1 e por isso a omitimos. Mostremos que 1 implica 2. A prova é construtiva e pode ser acompanhada através da Figura 5.1. Lembremos que a norma de um elemento $x=\left(x_{1}, x_{2}, x_{3}\right) \in \mathbb{R}^{3}$ é definida por $\|x\|=\sqrt{x_{1}^{2}+x_{2}^{2}+x_{3}^{2}}$. Lembremos ainda que $\mathbb{S}^{2}=\left\{x \in \mathbb{R}^{3} \mid\|x\|=1\right\}$. Se uma classe de homotopia $\beta \in\left[M, \mathbb{S}^{2}\right]$ não tem a propriedade de Borsuk-Ulam, por definição, $\beta$ tem um representante $g: M \rightarrow \mathbb{S}^{2}$ tal que para todo $x \in M$ vale

$$
g(\tau(x)) \neq g(x)
$$

Por esse motivo, o segmento de reta que une $g(\tau(x))$ a $-g(x)$ não passa pela origem de $\mathbb{R}^{3}$. Assim, está bem definida a homotopia

$$
H: M \times[0,1] \rightarrow \mathbb{S}^{2} \text { dada por } H(x, t)=\frac{(1-t) g(\tau(x))+t(-g(x))}{\|(1-t) g(\tau(x))+t(-g(x))\|} .
$$

Seja

$$
f: M \rightarrow \mathbb{S}^{2} \text { definida por } f(x)=-H\left(x, \frac{1}{2}\right)=\frac{g(x)-g(\tau(x))}{\|g(x)-g(\tau(x))\|} .
$$




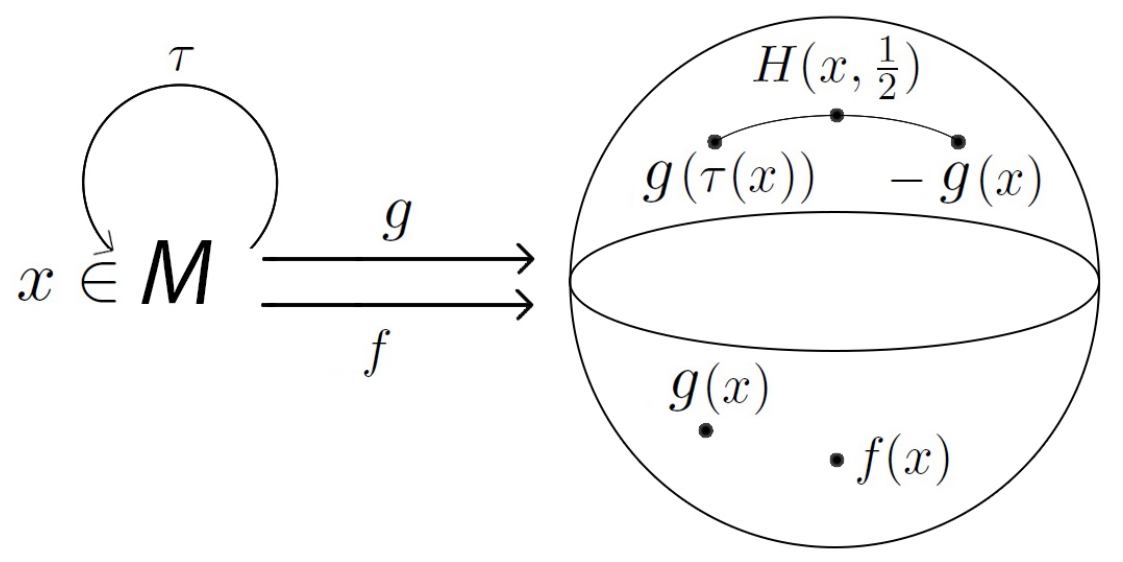

Figura 5.1: construção de $H$ ef.

Notemos que $-H(x, 1)=-\frac{-g(x)}{\|-g(x)\|}=g(x)$, e portanto $f \simeq g$. Logo, $\beta=[g]=[f]$. Por fim, mostremos que $f$ é $(\tau, A)$-equivariante. Seja $x \in M$. Temos

$$
f(\tau(x))=\frac{g(\tau(x))-g(\tau(\tau(x)))}{\|g(\tau(x))-g(\tau(\tau(x)))\|}=\frac{g(\tau(x))-g(x)}{\|g(\tau(x))-g(x)\|}=-\frac{g(x)-g(\tau(x))}{\|g(x)-g(\tau(x))\|}=A(f(x)) .
$$

Em [10, Lema 3.2], nós temos um resultado que trata sobre grau módulo 2 e aplicações de recobrimento e que será utilizado na demonstração do Lema 5.2.3. Transcrevemos aqui este resultado no caso particular em que os espaços envolvidos são superfícies fechadas.

Proposição 5.2.2. Consideremos o seguinte diagrama comutativo

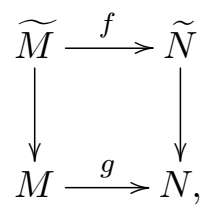

sendo que todos os espaços são superfícies fechadas, as flechas verticais são aplicações de recobrimento com mesmo número finito de folhas e $f$ é injetora nas fibras. Então vale a seguinte condição:

$$
\begin{aligned}
& f^{*}: \mathrm{H}^{2}\left(\tilde{N} ; \mathbb{Z}_{2}\right) \rightarrow \mathrm{H}^{2}\left(\widetilde{M} ; \mathbb{Z}_{2}\right) \text { é isomorfismo (resp. trivial) } \\
& \text { se, e somente se, } \\
& g^{*}: \mathrm{H}^{2}\left(N ; \mathbb{Z}_{2}\right) \rightarrow \mathrm{H}^{2}\left(M ; \mathbb{Z}_{2}\right) \text { é isomorfismo (resp. trivial). }
\end{aligned}
$$

Lema 5.2.3. Sejam $M$ uma superfície fechada, $\tau: M \rightarrow M$ uma involução livre de pontos fixos tal que o espaço de órbitas $M_{\tau}$ é não orientável e $f: M \rightarrow \mathbb{S}^{2}$ uma função $(\tau, A)$-equivariante. Vale a seguinte condição, sendo $\theta_{\tau}: \pi_{1}\left(M_{\tau}\right) \rightarrow \mathbb{Z}_{2}$ como definido em (5.1) e $\delta$ como definido em (5.2):

$$
f^{*}: \mathrm{H}^{2}\left(\mathbb{S}^{2} ; \mathbb{Z}_{2}\right) \rightarrow \mathrm{H}^{2}\left(M ; \mathbb{Z}_{2}\right) \text { é }\left\{\begin{array}{l}
\text { trivial, se } \theta_{\tau}(\delta)=\overline{0} \\
\text { isomorfismo, se } \theta_{\tau}(\delta)=\overline{1} .
\end{array}\right.
$$

Demonstração. Como $f: M \rightarrow \mathbb{S}^{2}$ é $(\tau, A)$-equivariante, então para cada $x \in M$, temos que $f\{x, \tau(x)\}=\{f(x),-f(x)\}$. Logo, $f$ se passa ao quociente, isto é, existe $g: M_{\tau} \rightarrow \mathbb{R P}^{2}$ tal que o 
seguinte diagrama é comutativo:

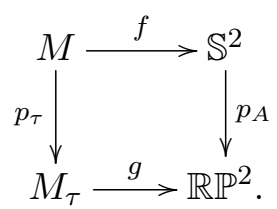

Seguindo o mesmo raciocínio que utilizamos na demonstração do Lema 1.4.1, a partir de (5.3), nós obtemos o seguinte diagrama comutativo:

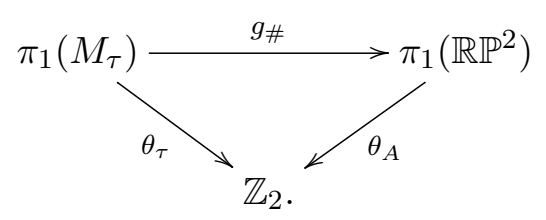

Como $\pi_{1}\left(\mathbb{R P}^{2}\right)$ é isomorfo a $\mathbb{Z}_{2}$ e $\theta_{A}$ é sobrejetora, então $\theta_{A}$ é isomorfismo, e portanto, salvo isomorfismo, nós podemos supor que $\theta_{\tau}=g_{\#}$. Usando o fato que o primeiro grupo de homologia com coeficientes inteiros é isomorfo ao abelianizado do grupo fundamental e que este isomorfismo é natural em relação a homomorfismos induzidos por funções contínuas, nós temos o seguinte diagrama comutativo, sendo as flechas verticais as projeções naturais:

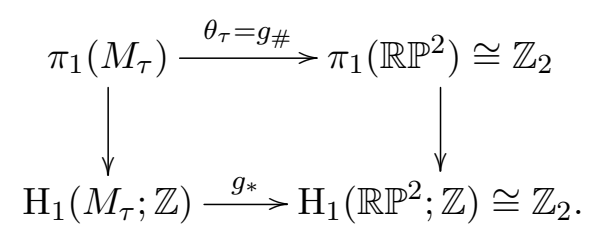

Notemos que

- Se $\chi\left(M_{\tau}\right)$ é par, então $\mathrm{H}_{1}\left(M_{\tau} ; \mathbb{Z}\right)=\mathbb{Z}_{2}[u] \oplus \mathbb{Z}[v] \oplus \mathbb{Z}\left[a_{1}\right] \oplus \cdots \oplus \mathbb{Z}\left[a_{2 n-1}\right] \oplus \mathbb{Z}\left[a_{2 n}\right]$.

- Se $\chi\left(M_{\tau}\right)$ é ímpar, então $\mathrm{H}_{1}\left(M_{\tau} ; \mathbb{Z}\right)=\mathbb{Z}_{2}[c] \oplus \mathbb{Z}\left[a_{1}\right] \oplus \cdots \oplus \mathbb{Z}\left[a_{2 n-1}\right] \oplus \mathbb{Z}\left[a_{2 n}\right]$.

De maneira resumida, nós podemos escrever

$$
\mathrm{H}_{1}\left(M_{\tau} ; \mathbb{Z}\right)=\mathbb{Z}_{2}[\delta] \oplus \mathbb{Z}\left[b_{1}\right] \oplus \cdots \oplus \mathbb{Z}\left[b_{k}\right]
$$

sendo que $k$ só depende de $\chi\left(M_{\tau}\right)$. Da comutatividade de (5.4), nós temos que

$$
g_{*}(\delta)=\left\{\begin{array}{l}
\overline{0}, \text { se } \theta_{\tau}(\delta)=\overline{0} \\
\overline{1}, \text { se } \theta_{\tau}(\delta)=\overline{1} .
\end{array}\right.
$$

Definimos alguns homomorfismos seguindo as flechas do seguinte diagrama:

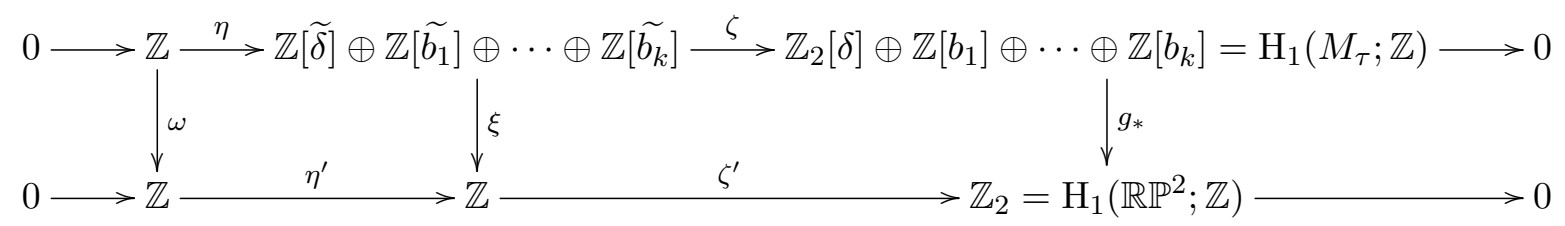

sendo $\eta(1)=2 \widetilde{\delta}, \eta^{\prime}(1)=2, \zeta(\widetilde{\delta})=\delta, \zeta\left(\widetilde{b_{i}}\right)=b_{i}, \zeta^{\prime}(1)=\overline{1}$,

$$
\omega(1)=\left\{\begin{array}{l}
0, \text { se } \theta_{\tau}(\delta)=\overline{0} \\
1, \text { se } \theta_{\tau}(\delta)=\overline{1}
\end{array}\right.
$$




$$
\xi(\tilde{\delta})=\left\{\begin{array}{l}
0, \text { se } g_{*}(\delta)=\overline{0} \\
1, \text { se } g_{*}(\delta)=\overline{1}
\end{array} \quad \xi\left(\tilde{b_{i}}\right)=\left\{\begin{array}{l}
0, \text { se } g_{*}\left(b_{i}\right)=\overline{0} \\
1, \text { se } g_{*}\left(b_{i}\right)=\overline{1}
\end{array}\right.\right.
$$

Usando (5.5), é fácil mostrar que com estas definições, o diagrama (5.6) é comutativo e por isso omitimos os cálculos. Notemos que as flechas horizontais são resoluções livres de $\mathrm{H}_{1}\left(M_{\tau} ; \mathbb{Z}\right)$ e $\mathrm{H}_{1}\left(\mathbb{R P}^{2} ; \mathbb{Z}\right)$, respectivamente. Tomamos o funtor $\operatorname{Hom}\left({ }_{-}, \mathbb{Z}_{2}\right)$ nestes homomorfismos e obtemos o diagrama comutativo dual:

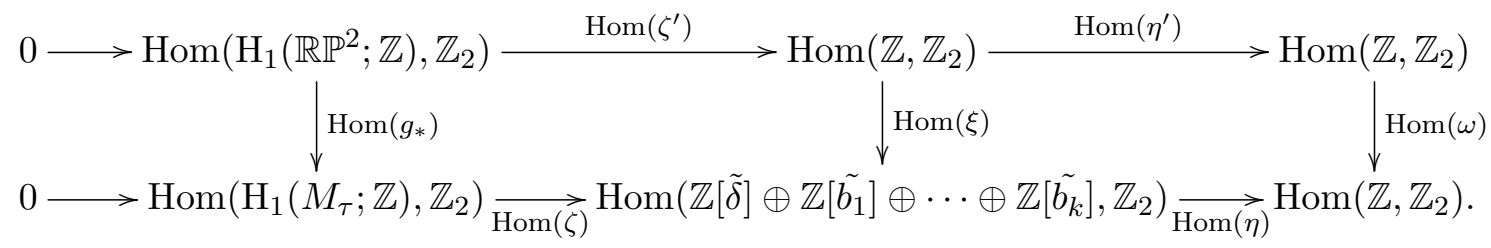

Notemos que o homomorfismo Hom $\left(\eta^{\prime}\right)$ é trivial. De fato, se $h: \mathbb{Z} \rightarrow \mathbb{Z}_{2}$ é um homomorfismo, então $\operatorname{Hom}\left(\eta^{\prime}\right)(h)(1)=\left(h \circ \eta^{\prime}\right)(1)=h(2)=2 h(1)=\overline{0}$. Analogamente, $\operatorname{Hom}(\eta)$ é trivial. Por definição $\operatorname{Ext}\left(\mathrm{H}_{1}\left(\mathbb{R P}^{2} ; \mathbb{Z}\right), \mathbb{Z}_{2}\right)=\operatorname{coker}\left(\operatorname{Hom}\left(\eta^{\prime}\right)\right)$ e $\operatorname{Ext}\left(\mathrm{H}_{1}\left(M_{\tau} ; \mathbb{Z}\right), \mathbb{Z}_{2}\right)=\operatorname{coker}(\operatorname{Hom}(\eta))$, e portanto, usando (5.8) nós obtemos o seguinte diagrama comutativo:

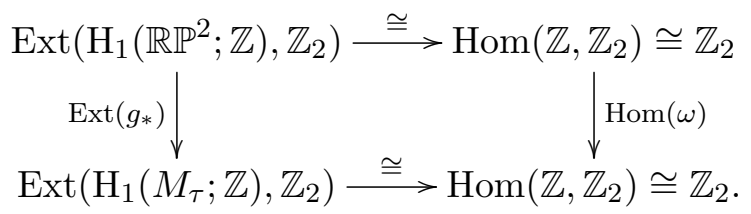

Seja $j: \mathbb{Z} \rightarrow \mathbb{Z}_{2}$ o homomorfismo definido por $j(1)=\overline{1}$. Por (5.7), temos que

$$
\operatorname{Hom}(\omega)(j)(1)=(j \circ w)(1)=\left\{\begin{array}{l}
j(0)=\overline{0}, \text { se } \theta_{\tau}(\delta)=\overline{0} \\
j(1)=\overline{1}, \text { se } \theta_{\tau}(\delta)=\overline{1}
\end{array}\right.
$$

Deste modo, por (5.9) e (5.10) nós temos que

$$
\operatorname{Ext}\left(g_{*}\right): \operatorname{Ext}\left(\mathrm{H}_{1}\left(\mathbb{R P}^{2} ; \mathbb{Z}\right), \mathbb{Z}_{2}\right) \rightarrow \operatorname{Ext}\left(\mathrm{H}_{1}\left(M_{\tau} ; \mathbb{Z}\right), \mathbb{Z}_{2}\right) \text { é }\left\{\begin{array}{l}
\text { trivial, se } \theta_{\tau}(\delta)=\overline{0} \\
\text { isomorfismo, se } \theta_{\tau}(\delta)=\overline{1}
\end{array}\right.
$$

Pelo Teorema dos coeficientes universais para cohomologia, nós temos o seguinte diagrama comutativo com linhas horizontais exatas:

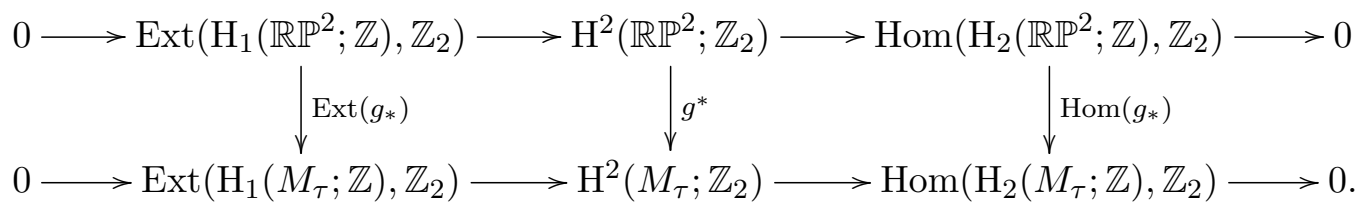

Como $\mathbb{R} \mathbb{P}^{2}$ e $M_{\tau}$ são não orientáveis, então $\mathrm{H}_{2}\left(\mathbb{R P}^{2} ; \mathbb{Z}\right)$ e $\mathrm{H}_{2}\left(M_{\tau} ; \mathbb{Z}\right)$ são triviais e consequentemente $\operatorname{Hom}\left(\mathrm{H}_{2}\left(\mathbb{R P}^{2} ; \mathbb{Z}\right), \mathbb{Z}_{2}\right)$ e $\operatorname{Hom}\left(\mathrm{H}_{2}\left(M_{\tau} ; \mathbb{Z}\right), \mathbb{Z}_{2}\right)$ são triviais. Logo, por $(5.11)$ e pela exatidão das linhas horizontais do diagrama (5.12), nós temos que

$$
g^{*}: \mathrm{H}^{2}\left(\mathbb{R P}^{2} ; \mathbb{Z}_{2}\right) \rightarrow \mathrm{H}^{2}\left(M_{\tau} ; \mathbb{Z}_{2}\right) \text { é }\left\{\begin{array}{l}
\text { trivial, se } \theta_{\tau}(\delta)=\overline{0} \\
\text { isomorfismo, se } \theta_{\tau}(\delta)=\overline{1}
\end{array}\right.
$$

Por fim, notemos que o diagrama (5.3) está nas hipóteses da Proposição 5.2.2. Portanto, segue por (5.13) que $f^{*}: \mathrm{H}^{2}\left(\mathbb{S}^{2} ; \mathbb{Z}_{2}\right) \rightarrow \mathrm{H}^{2}\left(M ; \mathbb{Z}_{2}\right)$ é $\left\{\begin{array}{l}\text { trivial, se } \theta_{\tau}(\delta)=\overline{0} \\ \text { isomorfismo, se } \theta_{\tau}(\delta)=\overline{1} \text {. }\end{array}\right.$ 


\subsubsection{Prova do Teorema 5.1.2}

Demonstração do Teorema 5.1.2. Vamos fazer a prova por contra positiva, ou seja, vamos mostrar que é verdadeira a seguinte afirmação:

Uma classe de homotopia $\beta \in\left[M, \mathbb{S}^{2}\right]$ não tem a propriedade de Borsuk-Ulam se, e somente se, $\beta$ é a classe de homotopia de uma função constante.

Primeiramente suponhamos que $\beta$ é a classe de homotopia de uma função constante e vamos mostrar que $\beta$ não tem a propriedade de Borsuk-Ulam. Como $M$ e $M_{\tau}$ são orientáveis, pelo Teorema 1.3.6, a tripla $\left(M, \tau ; \mathbb{R}^{2}\right)$ não tem a propriedade de Borsuk-Ulam. Logo, existe $g: M \rightarrow \mathbb{R}^{2}$ tal que $g(\tau(x)) \neq g(x)$ para todo $x \in M$. Seja $j: \mathbb{R}^{2} \rightarrow \mathbb{S}^{2}$ um mergulho topológico. Temos que a função $f=j \circ g: M \rightarrow \mathbb{S}^{2}$ é homotópica a uma função constante, uma vez que $\mathbb{R}^{2}$ é contrátil, e portanto, $\beta=[f]$, pois todas as funções constantes são homotópicas. Como $f(\tau(x)) \neq f(x)$ para todo $x \in M$, então $\beta$ não tem a propriedade de Borsuk-Ulam.

Reciprocamente, suponhamos que uma classe de homotopia $\beta \in\left[M, \mathbb{S}^{2}\right]$ não tem a propriedade de Borsuk-Ulam e vamos mostrar que $\beta$ é a classe de homotopia de uma função constante. Pelo Lema 5.2.1, $\beta$ tem um representante $f: M \rightarrow \mathbb{S}^{2}$ que é $(\tau, A)$-equivariante. Logo, o seguinte diagrama é comutativo:

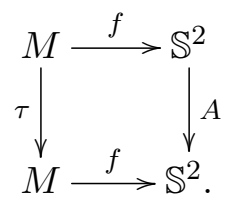

Tomando o segundo grupo de cohomologia com coeficientes inteiros, nós temos o seguinte diagrama comutativo:

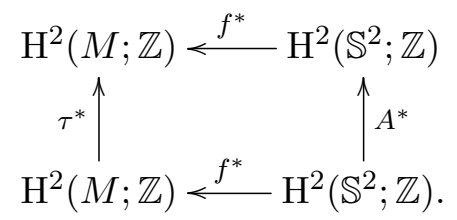

Como $M_{\tau}$ é orientável, então a involução $\tau$ é uma aplicação que preserva orientação, e portanto, $\tau^{*}$ é a identidade. Lembremos que $A^{*}$ é menos a identidade. Assim, se $z_{m}$ e $z_{s}$ são os respectivos geradores dos grupos cíclicos $\mathrm{H}^{2}(M ; \mathbb{Z})$ e $\mathrm{H}^{2}\left(\mathbb{S}^{2} ; \mathbb{Z}\right)$ e $d(f)$ é o grau de $f$, temos que

$$
d(f) z_{m}=f^{*}\left(z_{s}\right)=\left(\tau^{*} \circ f^{*}\right)\left(z_{s}\right) \stackrel{(5.14)}{=}\left(f^{*} \circ A^{*}\right)\left(z_{s}\right)=f^{*}\left(-z_{s}\right)=-f^{*}\left(z_{s}\right)=-d(f) z_{m}
$$

donde segue que $\operatorname{deg}(\beta)=d(f)=0$, sendo $\operatorname{deg}:\left[M, \mathbb{S}^{2}\right] \rightarrow \mathbb{Z}$ como definido no Teorema 5.1.1. Como deg é uma bijeção, então $\beta$ é a classe de homotopia de uma função constante.

\subsubsection{Prova do Teorema 5.1.3}

Lema 5.2.4. Sejam $M$ uma superfície fechada orientável e $\tau: M \rightarrow M$ uma involução livre de pontos fixos tal que o espaço de órbitas $M_{\tau}$ é não orientável. Valem as seguintes afirmaçôes, sendo $\theta_{\tau}: \pi_{1}\left(M_{\tau}\right) \rightarrow \mathbb{Z}_{2}$ como definido em (5.1) e $\delta$ como definido em (5.2):

- Se $\chi\left(M_{\tau}\right)$ é par, então $\theta_{\tau}(\delta)=\overline{0}$;

- Se $\chi\left(M_{\tau}\right)$ é impar, então $\theta_{\tau}(\delta)=\overline{1}$.

Ou equivalentemente, a seguinte afirmação é verdadeira:

$$
\chi\left(M_{\tau}\right) \text { é par (resp. impar) se, e somente se, } \theta_{\tau}(\delta)=\overline{0}(\text { resp. } \overline{1}) .
$$


Demonstração. Se $\chi\left(M_{\tau}\right)$ é par, então

$$
\pi_{1}\left(M_{\tau}\right)=\left\langle u, v, a_{1}, a_{2}, \cdots, a_{2 n-1}, a_{2 n} \mid u v u v^{-1}\left[a_{1}, a_{2}\right] \ldots\left[a_{2 n-1}, a_{2 n}\right]=1\right\rangle \quad \text { e } \delta=u .
$$

Suponhamos primeiramente que $\theta_{\tau}(v)=\overline{0}$. Como temos a sequência exata

$$
1 \longrightarrow \pi_{1}(M) \stackrel{\left(p_{\tau}\right)_{\#}}{\longrightarrow} \pi_{1}\left(M_{\tau}\right) \stackrel{\theta_{\tau}}{\longrightarrow} \mathbb{Z}_{2} \longrightarrow 1
$$

então existe um elemento $v^{\prime} \in \pi_{1}(M)$ tal que $\left(p_{\tau}\right)_{\#}\left(v^{\prime}\right)=v$. Como $v \in \pi_{1}\left(M_{\tau}\right)$ é uma classe de homotopia de laços que invertem orientação e como $p_{\tau}: M \rightarrow M_{\tau}$ é uma aplicação de recobrimento, então $v^{\prime}$ é uma classe de homotopia de laços que invertem orientação, o que é um absurdo, pois $M$ é orientável. Portanto, $\theta_{\tau}(v)=\overline{1}$. Como $u$ é uma classe de homotopia de laços que preservam orientação, então uv é uma classe de laços que invertem orientação, e repetindo o argumento anterior, temos que $\theta_{\tau}(u v)=\overline{1}$. Assim, temos que

$$
\theta_{\tau}(\delta)=\theta_{\tau}(u)=\theta_{\tau}(u)+2 \theta_{\tau}(v)=\theta_{\tau}(u)+\theta_{\tau}(v)+\theta_{\tau}(v)=\theta_{\tau}(u v)+\theta_{\tau}(v)=\overline{1}+\overline{1}=\overline{0} .
$$

Se $\chi\left(M_{\tau}\right)$ é ímpar, então

$$
\pi_{1}\left(M_{\tau}\right)=\left\langle c, a_{1}, a_{2}, \cdots, a_{2 n-1}, a_{2 n} \mid c^{2}\left[a_{1}, a_{2}\right] \ldots\left[a_{2 n-1}, a_{2 n}\right]=1\right\rangle \quad \text { e } \quad \delta=c .
$$

Como $c$ é uma classe de homotopia que representa laços de $M_{\tau}$ que invertem orientação, então $\theta_{\tau}(c)=\overline{1}$, pelo mesmo argumento que usamos anteriormente, e portanto, $\theta_{\tau}(\delta)=\overline{1}$.

Lema 5.2.5. Sejam $M$ uma superfície orientável, $\tau: M \rightarrow M$ uma involução livre de pontos fixos tal que o espaço de órbitas $M_{\tau}$ é não orientável. Se $f: M \rightarrow \mathbb{S}^{2}$ é uma função $(\tau, A)$-equivariante, então vale a seguinte condição, sendo que $d(f) \in \mathbb{Z}$ denota o grau de $f, \theta_{\tau}: \pi_{1}\left(M_{\tau}\right) \rightarrow \mathbb{Z}_{2}$ é como definido em (5.1) e $\delta$ é como definido (5.2):

$$
d(f) e ́\left\{\begin{array}{l}
\text { par, se } \theta_{\tau}(\delta)=\overline{0} \\
\text { impar, se } \theta_{\tau}(\delta)=\overline{1} .
\end{array}\right.
$$

Demonstração. Por [9, Capítulo VI, Corolário 10.26], nós temos o seguinte diagrama comutativo com linhas horizontais exatas:

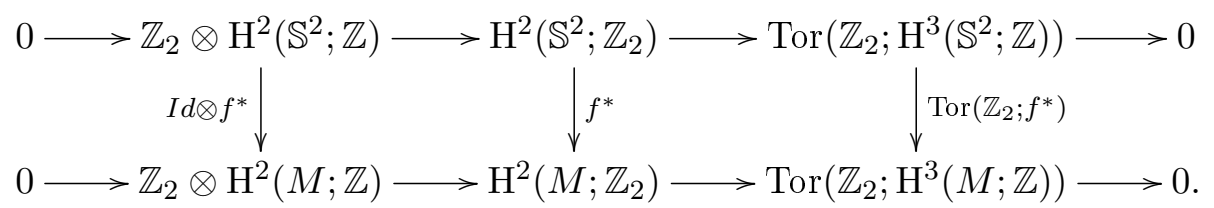

Como $\mathbb{S}^{2}$ e $M$ são superfícies fechadas, então $\mathrm{H}^{3}\left(\mathbb{S}^{2} ; \mathbb{Z}\right)$ e $\mathrm{H}^{3}(M ; \mathbb{Z})$ são triviais, e portanto são triviais $\operatorname{Tor}\left(\mathbb{Z}_{2} ; \mathrm{H}^{3}\left(\mathbb{S}^{2} ; \mathbb{Z}\right)\right)$ e $\operatorname{Tor}\left(\mathbb{Z}_{2} ; \mathrm{H}^{3}(M ; \mathbb{Z})\right)$. Logo, como $f$ é $(\tau, A)$-equivariante, segue do Lema 5.2 .3 e do diagrama (5.15) que

$$
I d \otimes f^{*}: \mathbb{Z}_{2} \otimes \mathrm{H}^{2}\left(\mathbb{S}^{2} ; \mathbb{Z}\right) \rightarrow \mathbb{Z}_{2} \otimes \mathrm{H}^{2}(M ; \mathbb{Z}) \text { é }\left\{\begin{array}{l}
\text { trivial, se } \theta_{\tau}(\delta)=\overline{0} \\
\text { isomorfismo, se } \theta_{\tau}(\delta)=\overline{1}
\end{array}\right.
$$

Lembremos que se $z_{s}$ e $z_{m}$ são os respectivos geradores dos grupos cíclicos infinitos $\mathrm{H}^{2}\left(\mathbb{S}^{2} ; \mathbb{Z}\right)$ e $\mathrm{H}^{2}(M ; \mathbb{Z})$, então $\overline{1} \otimes z_{s}$ e $\overline{1} \otimes z_{m}$ são os respectivos geradores dos grupos $\mathbb{Z}_{2} \otimes \mathrm{H}^{2}\left(\mathbb{S}^{2} ; \mathbb{Z}\right)$ e $\mathbb{Z}_{2} \otimes \mathrm{H}^{2}(M ; \mathbb{Z})$, os quais são cíclicos de ordem 2 . Assim, temos que

$$
I d \otimes f^{*}\left(\overline{1} \otimes z_{s}\right)=I d(\overline{1}) \otimes f^{*}\left(z_{s}\right)=\overline{1} \otimes d(f) z_{m}=d(f)\left(\overline{1} \otimes z_{s}\right)=\overline{d(f)} \otimes z_{s}
$$


e portanto, segue de (5.16) que

$$
d(f) \text { é }\left\{\begin{array}{l}
\text { par, se } \theta_{\tau}(\delta)=\overline{0} \\
\text { ímpar, se } \theta_{\tau}(\delta)=\overline{1}
\end{array}\right.
$$

Sejam $M$ uma superfície fechada orientável e $\tau: M \rightarrow M$ uma involução livre de pontos fixos tal que o espaço de órbitas $M_{\tau}$ é não orientável. Notemos que neste caso, $\tau$ é um homeomorfismo que inverte orientação e portanto, $\tau^{*}: \mathrm{H}^{2}(M ; \mathbb{Z}) \rightarrow \mathrm{H}^{2}(M ; \mathbb{Z})$ é menos a identidade. Lembremos que $A^{*}: \mathrm{H}^{2}\left(\mathbb{S}^{2} ; \mathbb{Z}\right) \rightarrow \mathrm{H}^{2}\left(\mathbb{S}^{2} ; \mathbb{Z}\right)$ também é menos a identidade. Assim, se considerarmos $\mathbb{Z}_{2} \cong\left\{I d_{M}, \tau\right\} \cong\left\{I d_{\mathbb{S}^{2}}, A\right\}$, então $\mathrm{H}^{2}(M ; \mathbb{Z})$ e $\mathrm{H}^{2}\left(\mathbb{S}^{2} ; \mathbb{Z}\right)$ são isomorfos como $\mathbb{Z}_{2}$-módulos. Estas são as hipóteses necessárias para que seja verdadeiro o seguinte resultado, o qual está enunciado em [8, Teorema 4.11] para situações mais gerais.

Proposição 5.2.6. Sejam $M$ uma superfície fechada orientável e $\tau: M \rightarrow M$ uma involução livre de pontos fixos tal que o espaço de órbitas $M_{\tau}$ é não orientável. Valem as seguintes condiçôes:

1. Existe uma função $f: M \rightarrow \mathbb{S}^{2}$ que é $(\tau, A)$-equivariante;

2. Se d é um número inteiro tal que $d \equiv d(f) \bmod 2$, então existe uma função $f^{\prime}: M \rightarrow \mathbb{S}^{2}$ que é $(\tau, A)$-equivariante e tal que $d\left(f^{\prime}\right)=d$;

3. Se $f^{\prime}: M \rightarrow \mathbb{S}^{2}$ é uma função $(\tau, A)$-equivariante, então $d\left(f^{\prime}\right) \equiv d(f) \bmod 2$.

Demonstração do Teorema 5.1.3. Notemos que por contra positiva, para que o resultado fique demonstrado, basta mostrarmos que são verdadeiras as seguintes afirmações:

1. Se $\chi\left(M_{\tau}\right)$ é par, então $\beta$ não tem a propriedade de Borsuk-Ulam se, e somente se, $\operatorname{deg}(\beta)$ é par.

2. Se $\chi\left(M_{\tau}\right)$ é ímpar, então $\beta$ não tem a propriedade de Borsuk-Ulam se, e somente se, $\operatorname{deg}(\beta)$ é ímpar.

Notemos que pelos Lemas 5.2.1 e 5.2.4, as afirmações $1^{\prime}$ e $2^{\prime}$ são equivalentes as seguintes afirmações:

$1^{\prime}$. Se $\theta_{\tau}(\delta)=\overline{0}$, então $\beta$ tem um representante $(\tau, A)$-equivariante se, e somente se, $\operatorname{deg}(\beta)$ é par.

$2^{\prime}$. Se $\theta_{\tau}(\delta)=\overline{1}$, então $\beta$ tem um representante $(\tau, A)$-equivariante se, e somente se, $\operatorname{deg}(\beta)$ é ímpar.

Vamos provar $1^{\prime}$ (resp. 2'). Seja $\beta \in\left[M, \mathbb{S}^{2}\right]$ uma classe de homotopia. Como $M$ é orientável e $M_{\tau}$ é não orientável, pela Proposição 5.2.6, item 1, existe uma função $f: M \rightarrow \mathbb{S}^{2}$ que é $(\tau, A)$ equivariante. Como $\theta_{\tau}(\delta)=\overline{0}$ (resp. $\theta_{\tau}(\delta)=\overline{1}$ ), pelo Lema 5.2.5, temos que $d(f)$ é par (resp. ímpar).

Se $\beta$ tem um representante $f^{\prime}: M \rightarrow \mathbb{S}^{2}$ que é $(\tau, A)$-equivariante, pela Proposição 5.2.6, item 3, então $\operatorname{deg}(\beta)=d\left(f^{\prime}\right) \equiv d(f) \bmod 2$, donde segue $\operatorname{deg}(\beta)$ é par (resp. ímpar).

Reciprocamente, se $\operatorname{deg}(\beta)=d$ é par (resp. ímpar), como $d \equiv d(f) \bmod 2$, pela Proposição 5.2.6, item 2 , existe uma função $f^{\prime}: M \rightarrow \mathbb{S}^{2}$ que é $(\tau, A)$-equivariante e $d\left(f^{\prime}\right)=d$. Como deg é bijeção, de acordo com o Teorema 5.1.1, então $\beta=\left[f^{\prime}\right]$. 


\subsubsection{Prova do Teorema 5.1.4}

Demonstração do teorema 5.1.4. Como $M$ é não orientável, pelo Teorema 5.1.1, o conjunto $\left[M, \mathbb{S}^{2}\right]$ tem dois elementos. Denotamos tais elementos por $\beta_{0}$ e $\beta_{1}$, sendo $\beta_{0}$ a classe de homotopia de uma função constante. Primeiramente, seja $\beta \in\left[M, \mathbb{S}^{2}\right]$ uma classe de homotopia que não tem a propriedade de Borsuk-Ulam e vamos mostrar que valem as seguintes afirmações:

(a) Se $\theta_{\tau}(\delta)=\overline{0}$, então $\beta=\beta_{0}$;

(b) Se $\theta_{\tau}(\delta)=\overline{1}$, então $\beta=\beta_{1}$.

Pelo Lema 5.2.1, existe uma função $f: M \rightarrow \mathbb{S}^{2}$ que representa $\beta$ e que é $(\tau, A)$-equivariante. Pelo Lema 5.2.3, temos que

$$
f^{*}: \mathrm{H}^{2}\left(\mathbb{S}^{2} ; \mathbb{Z}_{2}\right) \rightarrow \mathrm{H}^{2}\left(M ; \mathbb{Z}_{2}\right) \text { é }\left\{\begin{array}{l}
\text { trivial, se } \theta_{\tau}(\delta)=\overline{0} \\
\text { isomorfismo, se } \theta_{\tau}(\delta)=\overline{1}
\end{array}\right.
$$

Lembremos que $f^{*}\left(\bar{z}_{s}\right)=d(f, 2) \bar{z}_{m}$, sendo $\bar{z}_{s}$ e $\bar{z}_{m}$ os respectivos geradores dos grupos $\mathrm{H}^{2}\left(\mathbb{S}^{2} ; \mathbb{Z}_{2}\right)$ e $\mathrm{H}^{2}\left(M ; \mathbb{Z}_{2}\right)$, os quais são cíclicos de ordem 2 , e $d(f, 2) \in \mathbb{Z}_{2}$. Lembremos ainda que $\overline{\operatorname{deg}}(\beta)=d(f, 2)$, de acordo com a notação do Teorema 5.1.1. Logo, por (5.17) temos que

$$
\overline{\operatorname{deg}}(\beta)=\left\{\begin{array}{l}
\overline{0}, \text { se } \theta_{\tau}(\delta)=\overline{0} \\
\overline{1}, \text { se } \theta_{\tau}(\delta)=\overline{1}
\end{array}\right.
$$

As afirmações $(a)$ e $(b)$ seguem de $(5.18)$ e do fato que $\overline{\operatorname{deg}}$ é bijeção, $\overline{\operatorname{deg}}\left(\beta_{0}\right)=\overline{0}$ e $\overline{\operatorname{deg}}\left(\beta_{1}\right)=\overline{1}$.

Mostremos agora que existe uma classe de homotopia $\beta$ que não tem a propriedade de BorsukUlam. Como $M$ é não orientável, pela Teorema 1.3.7, a tripla $\left(M, \tau ; \mathbb{S}^{2}\right)$ não tem a propriedade de Borsuk-Ulam, isto é, existe uma função $f: M \rightarrow \mathbb{S}^{2}$ tal que $f(\tau(x)) \neq f(x)$, para todo $x \in M$. Assim, a classe de homotopia $\beta=[f] \in\left[M, \mathbb{S}^{2}\right]$ não tem a propriedade de Borsuk-Ulam.

Até esta parte da demonstração, nós podemos concluir que existe um elemento no conjunto $\left[M, \mathbb{S}^{2}\right]$ que não tem a propriedade de Borsuk-Ulam e este elemento é único. Novamente, como o conjunto $\left[M, \mathbb{S}^{2}\right]$ tem dois elementos, então por contra-positiva, existe apenas uma classe de homotopia $\beta \in\left[M, \mathbb{S}^{2}\right]$ que tem a propriedade de Borsuk-Ulam. Segue por $(a)$ e $(b)$, novamente por contrapositiva, que são verdadeiras as afirmações 1 e 2 do enunciado do resultado.

\subsection{Os $\operatorname{casos}\left(M, \tau ; \mathbb{R P}^{2}\right)$}

O objetivo desta seção é estudar a propriedade de Borsuk-Ulam para uma certa família de funções que têm como contra-domínio $\mathbb{R} \mathbb{P}^{2}$. Mais precisamente, dada uma involução livre de pontos fixos $\tau: M \rightarrow M$, nós vamos classificar, em relação a propriedade de Borsuk-Ulam, as classes de homotopia $\alpha \in\left[M, \mathbb{R P}^{2}\right]$ tal que $\alpha$ tem um representante que induz o homomorfismo trivial a nível de grupo fundamental (notemos que neste caso qualquer representante de $\alpha$ induz o homomorfismo trivial a nível de grupo fundamental).

Vamos fazer uma construção geométrica que será usada nas demonstrações dos Teoremas 5.1.5 e 5.1.6, as quais faremos nas Subseções 5.3.1 e 5.3.2, respectivamente. Ainda nesta seção, enunciaremos e provaremos dois resultados que também serão úteis.

Lema 5.3.1. Sejam $M$ uma superfície fechada e $\tau: M \rightarrow M$ uma involução livre de pontos fixos. Suponhamos que uma classe de homotopia $\alpha \in\left[M, \mathbb{R P}^{2}\right]$ tem um representante que induz o homomorfismo trivial no nível de grupo fundamental e que a não tem a propriedade de Borsuk-Ulam. Então, existe uma função $f: M \rightarrow \mathbb{S}^{2}$ tal que

$$
f(\tau(x)) \neq f(x) \quad \text { e } \quad f(\tau(x)) \neq-f(x), \quad \text { para todo } x \in M
$$


e tal que $\alpha=\left[p_{A} \circ f\right]$, sendo $p_{A}: \mathbb{S}^{2} \rightarrow \mathbb{R P}^{2}$ o recobrimento duplo induzido pela involução antipodal $A: \mathbb{S}^{2} \rightarrow \mathbb{S}^{2}$.

Demonstração. Por hipótese, existe uma função $g: M \rightarrow \mathbb{R} \mathbb{P}^{2}$ que representa $\alpha$, tal que valem as seguintes condições:

1. $g(\tau(x)) \neq g(x)$, para todo $x \in M$;

2. $g_{\#}: \pi_{1}(M) \rightarrow \pi_{1}\left(\mathbb{R P}^{2}\right)$ é trivial.

Por 2., existe um levantamento de $g$ pelo recobrimento $p_{A}: \mathbb{S}^{2} \rightarrow \mathbb{R P}^{2}$, isto é, existe uma função $f: M \rightarrow \mathbb{S}^{2}$, tal que o seguinte diagrama é comutativo:

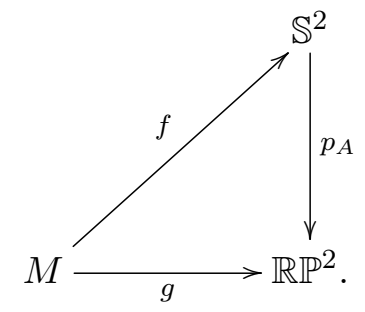

Da comutatividade deste diagrama e por 1 ., segue que para todo $x \in M$ vale

$$
f(\tau(x)) \neq f(x) \quad \text { e } \quad f(\tau(x)) \neq-f(x) .
$$

Também da comutatividade do diagrama segue que $\alpha=[g]=\left[p_{A} \circ f\right]$.

Lema 5.3.2. Sejam $M$ uma superfície fechada, $\tau: M \rightarrow M$ uma involução livre de pontos fixos $e$ $\mathcal{A}_{2}\left(\mathbb{S}^{2}\right)=\left\{(x, y) \in \mathbb{S}^{2} \times \mathbb{S}^{2} \mid x \neq \pm y\right\}$. Consideremos $\tau_{1}: \mathcal{A}_{2}\left(\mathbb{S}^{2}\right) \rightarrow \mathcal{A}_{2}\left(\mathbb{S}^{2}\right)$ a involução definida por $\tau_{1}(x, y)=(y, x)$ e $\mathcal{B}_{2}\left(\mathbb{S}^{2}\right)=\frac{\mathcal{A}_{2}\left(\mathbb{S}^{2}\right)}{\sim}$ o espaço de órbitas. Se existe uma função $f: M \rightarrow \mathbb{S}^{2}$ tal que $f(\tau(x)) \neq \pm f(x)$ para todo $x \in M$, então existe o seguinte diagrama comutativo (omitimos os pontos bases):

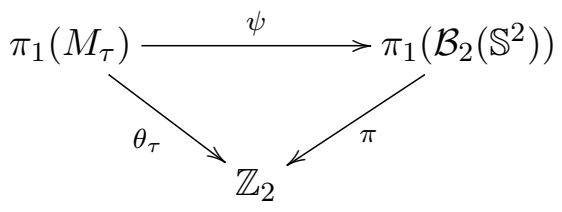

sendo $\pi: \pi_{1}\left(\mathcal{B}_{2}\left(\mathbb{S}^{2}\right)\right) \rightarrow \mathbb{Z}_{2} \cong \frac{\pi_{1}\left(\mathcal{B}_{2}\left(\mathbb{S}^{2}\right)\right)}{\left(p_{\tau_{1}}\right)_{\#}\left(\pi_{1}\left(\mathcal{A}_{2}\left(\mathbb{S}^{2}\right)\right)\right)}$ a projeção natural.

Demonstração. Suponhamos que $f: M \rightarrow \mathbb{S}^{2}$ seja uma função tal que $f(\tau(x)) \neq \pm f(x)$, para todo $x \in M$. Por este motivo, está bem definida a seguinte função:

$$
\begin{aligned}
F: M & \longrightarrow \mathcal{A}_{2}\left(\mathbb{S}^{2}\right) \\
x & \longmapsto(f(\tau(x)), f(x)) .
\end{aligned}
$$

Notemos que para cada $x \in M$, temos

$$
F(\tau(x))=(f(\tau(\tau(x))), f(\tau(x)))=(f(x), f(\tau(x)))=\tau_{1}(F(x))
$$

ou seja, $F$ é $\left(\tau, \tau_{1}\right)$-equivariante. Portanto, existe $\bar{F}: M_{\tau} \rightarrow \mathcal{B}_{2}\left(\mathbb{S}^{2}\right)$ tal que o seguinte diagrama é comutativo:

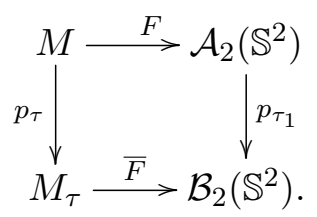

A demonstração daqui em diante segue utilizando exatamente os mesmos argumentos da primeira parte da demonstração do Lema 1.4.1, e por isso a omitimos. 
Lema 5.3.3. Sejam $M$ uma superfície fechada, $\tau: M \rightarrow M$ uma involução livre de pontos fixos e $\alpha \in\left[M, \mathbb{R P}^{2}\right]$ a classe de homotopia de uma função constante. Se $\left(M, \tau ; \mathbb{R}^{2}\right)$ não tem a propriedade de Borsuk-Ulam, então $\alpha$ não tem a propriedade de Borsuk-Ulam com respeito a $\tau$.

Demonstração. A prova é construtiva e pode ser acompanhada através da Figura 5.2. Seja $\mathbb{D}^{2}$ o disco fechado unitário de $\mathbb{R}^{2}$. Por hipótese, $\left(M, \tau ; \mathbb{R}^{2}\right)$ não tem a propriedade de Borsuk-Ulam e como $\mathbb{R}^{2}$ mergulha em $\mathbb{D}^{2}$, então o mesmo ocorre com a tripla $\left(M, \tau ; \mathbb{D}^{2}\right)$. Portanto, existe uma função $h: M \rightarrow \mathbb{D}^{2}$ tal que para todo $x \in M$ vale

$$
h(\tau(x)) \neq h(x) .
$$

Seja $j: \mathbb{D}^{2} \rightarrow \mathbb{S}^{2}$ um mergulho topológico tal que a imagem esteja contida no interior de um hemisfério de $\mathbb{S}^{2}$. Assim, a função $f=j \circ h: M \rightarrow \mathbb{S}^{2}$ é tal que para todo $x \in M$ vale

$$
f(\tau(x)) \neq \pm f(x) .
$$

Por fim, seja $g=p_{A} \circ f: M \rightarrow \mathbb{R P}^{2}$. Então, para todo $x \in M$ vale

$$
g(\tau(x)) \neq g(x) .
$$

Como $g=p_{A} \circ j \circ h$, então $g$ é homotópica a uma função constante e portanto, $g$ é um representante da classe de homotopia $\alpha$. Logo, $\alpha$ não tem a propriedade de Borsuk-Ulam.

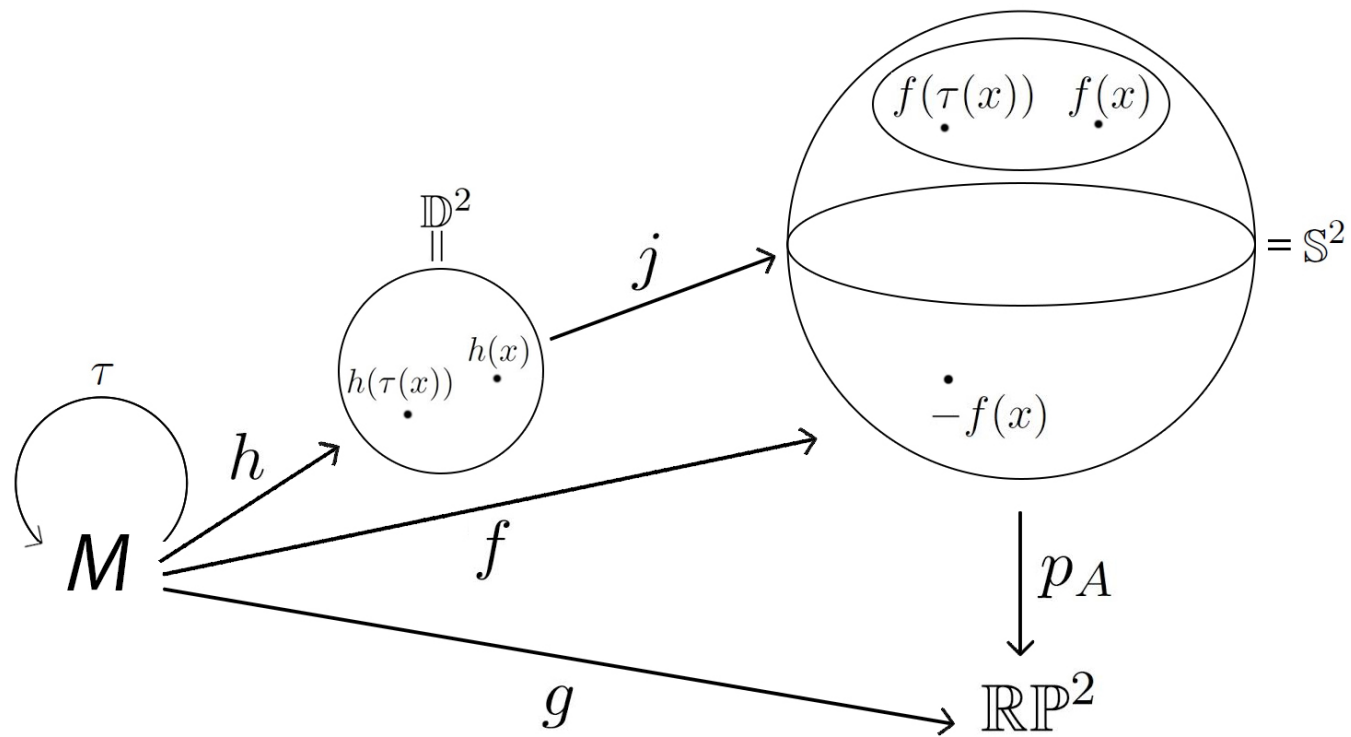

Figura 5.2: construção de $g$.

\subsubsection{Prova do Teorema 5.1.5}

Demonstração do Teorema 5.1.5. Por contra positiva, a tese do teorema pode ser reescrita do seguinte modo:

$\alpha$ não tem a propriedade de Borsuk-Ulam se, e somente se, as seguintes condições são satisfeitas:

$1^{\prime} . \alpha$ é a classe de homotopia de uma função constante;

$2^{\prime}$. o espaço de órbitas $M_{\tau}$ é orientável ou $M_{\tau}$ é não orientável e $\chi\left(M_{\tau}\right)$ é par. 
Vamos provar que estas afirmações são verdadeiras.

Suponhamos que $\alpha$ não tem a propriedade de Borsuk-Ulam. Pelo Lema 5.3.1, existe $f: M \rightarrow \mathbb{S}^{2}$ tal que $\alpha=\left[p_{A} \circ f\right]$ e tal que para todo $x \in M$ vale

$$
f(\tau(x)) \neq f(x) \quad \text { e } \quad f(\tau(x)) \neq-f(x) .
$$

Seja $\beta \in\left[M, \mathbb{S}^{2}\right]$ a classe de homotopia de $f$. Notemos que $\beta$ não tem a propriedade de BorsukUlam. Com argumentos geométricos análogos aos que foram usados na demonstração do Lema 5.2.1, podemos mostrar que estão bem definidas as homotopias $H, G: M \times[0,1] \rightarrow \mathbb{S}^{2}$, dadas por

$$
H(x, t)=\frac{(1-t) f(\tau(x))+t(-f(x))}{\|(1-t) f(\tau(x))+t(-f(x))\|} \quad \text { e } \quad G(x, t)=\frac{(1-t) f(\tau(x))+t f(x)}{\|(1-t) f(\tau(x))+t f(x)\|} .
$$

Para cada $t \in[0,1]$, sejam $h_{t}, g_{t}: M \rightarrow \mathbb{S}^{2}$ definidas por

$$
h_{t}(x)=H(x, t) \quad \text { e } \quad g_{t}(x)=G(x, t) .
$$

Notemos que

$$
A \circ f=h_{1} \simeq h_{0}=f \circ \tau=g_{0} \simeq g_{1}=f .
$$

Logo, $f$ é homotópica a $A \circ f$, ou equivalentemente, o seguinte diagrama é homotópico comutativo:

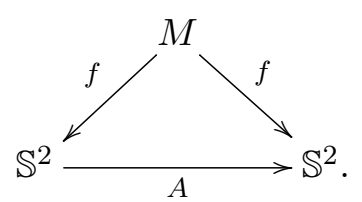

Tomando o segundo grupo de cohomologia com coeficientes inteiros, obtemos o seguinte diagrama comutativo:

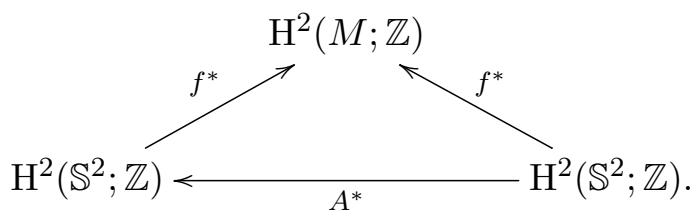

Sejam $z_{s}$ e $z_{m}$ os geradores dos grupos cíclicos $\mathrm{H}^{2}\left(\mathbb{S}^{2} ; \mathbb{Z}\right)$ e $\mathrm{H}^{2}(M ; \mathbb{Z})$, respectivamente, e $d(f) \in \mathbb{Z}$ o grau de $f$. Temos que

$$
d(f) z_{m}=f^{*}\left(z_{s}\right)=\left(f^{*} \circ A^{*}\right)\left(z_{s}\right)=f^{*}\left(-z_{s}\right)=-f^{*}\left(z_{s}\right)=-d(f) z_{m}
$$

e portanto, utilizando a notação e o resultado do Teorema 5.1.1, temos que $\operatorname{deg}(\beta)=d(f)=0$, donde segue que $\beta$ é a classe de homotopia de uma função constante. Como $\alpha=\left[p_{A} \circ f\right]$, então $\alpha$ é a classe de homotopia da função constante, ou seja, vale $1^{\prime}$. Como $M$ é orientável e $\beta$ não tem a propriedade de Borsuk-Ulam, segue dos Teoremas 5.1.2 e 5.1.3 que vale $2^{\prime}$.

Reciprocamente, suponhamos que valha $1^{\prime}$ e $2^{\prime}$. Como $\tau: M \rightarrow M$ é uma involução livre de pontos fixos, então, por [1, Capítulo 7, Problema 14], temos que $\frac{\chi(M)}{2}=\chi\left(M_{\tau}\right)$. Assim, segue da hipótese de $M$ ser orientável, por $2^{\prime}$ e pelo Teorema 1.3.6 que a tripla $\left(M, \tau ; \mathbb{R}^{2}\right)$ não tem a propriedade de Borsuk-Ulam. Como $\alpha$ é a classe de homotopia de uma função constante, segue pelo Lema 5.3.3 que $\alpha$ não tem a propriedade de Borsuk-Ulam.

\subsubsection{Prova do Teorema 5.1.6}

Demonstração do Teorema 5.1.6 item 1. Notemos que por contra positiva, o item 1 é equivalente a seguinte afirmação: 
$1^{\prime}$. Se $\theta_{\tau}(\delta)=\overline{0}$, então $\alpha$ não tem a propriedade de Borsuk-Ulam se, e somente se, $\alpha$ é a classe de homotopia de uma função constante.

Vamos provar 1'. Suponhamos que $\alpha$ não tem a propriedade de Borsuk-Ulam. Pelo Lema 5.3.1, existe $f: M \rightarrow \mathbb{S}^{2}$ tal que $\alpha=\left[p_{A} \circ f\right]$ e tal que para todo $x \in M$ vale $f(\tau(x)) \neq \pm f(x)$. Notemos que a classe de homotopia $\beta=[f] \in\left[M, \mathbb{S}^{2}\right]$ não tem a propriedade de Borsuk-Ulam. Como $M$ é não orientável, pelo Teorema 5.1.1, o conjunto $\left[M, \mathbb{S}^{2}\right]$ tem dois elementos. Como $\theta_{\tau}(\delta)=\overline{0}$, pelo Teorema 5.1.4, $\beta$ é a classe de homotopia de uma função constante e portanto, $f$ é homotópica a uma função constante. Logo, $\alpha=\left[p_{A} \circ f\right]$ é a classe de homotopia de uma função constante.

Reciprocamente, seja $\alpha$ a classe de homotopia de uma função constante. Como $M$ é não orientável e $\theta_{\tau}(\delta)=\overline{0}$, pelo Teorema 1.3.6, a tripla $\left(M, \tau ; \mathbb{R}^{2}\right)$ não tem a propriedade de Borsuk-Ulam. Segue pelo Lema 5.3.3, que $\alpha$ não tem a propriedade de Borsuk-Ulam.

Demonstração do Teorema 5.1.6 item 2. Vamos fazer a prova por contradição. Suponhamos então que $\theta_{\tau}(\delta)=\overline{1}, \alpha \in\left[M, \mathbb{R P}^{2}\right]$ seja uma classe de homotopia que tem um representante que induz o homomorfismo trivial e por absurdo, suponhamos que $\alpha$ não tem a propriedade de BorsukUlam. Pelo Lema 5.3.1, existe $f: M \rightarrow \mathbb{S}^{2}$ tal que $\alpha=\left[p_{A} \circ f\right]$ e tal que para todo $x \in M$ vale $f(\tau(x)) \neq \pm f(x)$. Logo, pelo Lema 5.3.2, temos o seguinte diagrama comutativo:

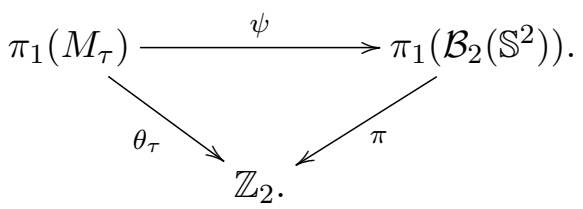

No apêndice C, Teorema C.0.22, está demonstrado que $\pi_{1}\left(\mathcal{B}_{2}\left(\mathbb{S}^{2}\right)\right)=\left\langle\sigma \mid \sigma^{4}=1\right\rangle$ e que $\pi: \pi_{1}\left(\mathcal{B}_{2}\left(\mathbb{S}^{2}\right)\right) \rightarrow \mathbb{Z}_{2}$ é definida por $\pi(\sigma)=\overline{1}$.

Se $\chi\left(M_{\tau}\right)$ é par, então

$$
\pi_{1}\left(M_{\tau}\right)=\left\langle u, v, a_{1}, a_{2}, \cdots, a_{2 n-1}, a_{2 n} \mid u v u v^{-1}\left[a_{1}, a_{2}\right] \ldots\left[a_{2 n-1}, a_{2 n}\right]=1\right\rangle \quad \text { e } \quad \delta=u .
$$

Como $\psi$ é homomorfismo, então

$$
\psi\left(u v u v^{-1}\left[a_{1}, a_{2}\right] \ldots\left[a_{2 n-1}, a_{2 n}\right]\right)=1 .
$$

Como $\pi_{1}\left(\mathcal{B}_{2}\left(\mathbb{S}^{2}\right)\right)$ é abeliano, a igualdade acima se reduz a

$$
\psi(u)^{2}=1 .
$$

Portanto, $\psi(u)=\sigma^{t}$, sendo $t=0$ ou $t=2$. Da comutatividade do diagrama acima, temos a seguinte igualdade em $\mathbb{Z}_{2}$ :

$$
\overline{1}=\theta_{\tau}(\delta)=\theta_{\tau}(u)=\pi(\psi(u))=\pi\left(\sigma^{t}\right)=t \pi(\sigma)=\bar{t}=\overline{0},
$$

o que é um absurdo. Portanto, $\alpha$ tem a propriedade de Borsuk-Ulam.

Se $\chi\left(M_{\tau}\right)$ é ímpar, então

$$
\pi_{1}\left(M_{\tau}\right)=\left\langle c, a_{1}, a_{2}, \ldots, a_{2 n-1}, a_{2 n}=1 \mid c^{2}\left[a_{1}, a_{2}\right] \ldots\left[a_{2 n-1}, a_{2 n}\right]\right\rangle \quad \text { e } \quad \delta=c .
$$

A argumentação é totalmente análoga ao caso anterior e por isso a omitimos. 


\section{Capítulo 6}

\section{A propriedade de Borsuk-Ulam para ações de $\mathbb{Z}_{p}$}

\subsection{Introdução}

Em todo este capítulo, $p$ denotará um número inteiro primo positivo.

$\mathrm{Na}$ Introdução deste trabalho, nós demos a definição da propriedade de Borsuk-Ulam para uma tripla $(M, \tau ; N)$, sendo $\tau: M \rightarrow N$ uma involução livre de pontos fixos (Definição 0.0.1). Neste último capítulo, nós vamos fazer uma generalização desta ideia para ações livres de $\mathbb{Z}_{p}$ (Definição 6.2.3). A nova definição vai coincidir com a Definição 0.0.1 no caso em que $p=2$. Vamos mostrar que diferente dos Teoremas 1.3.6 e 1.3.7, mantendo o domínio e o contra-domínio sendo superfícies fechadas, mas considerando $p \neq 2$, então nenhuma tripla terá a propriedade de Borsuk-Ulam (Teorema 6.2.5). Observemos que também neste caso $p \neq 2$, se coloca a questão de estudar o problema de classificar classes de homotopia que têm a propriedade de Borsuk-Ulam com respeito a estas ações, o qual poderá ser um tópico de uma pesquisa futura.

\subsection{Ações livre de $\mathbb{Z}_{p}$ e a propriedade de Borsuk-Ulam}

Definição 6.2.1. Dada $\varrho: \mathbb{Z}_{p} \times M \rightarrow M$ uma ação livre de $\mathbb{Z}_{p}$ em uma superfície fechada $M$, a função $\tau: M \rightarrow M$ definida por $\tau(x)=\varrho(\overline{1}, x)$ é denominada de gerador de $\varrho$.

Notemos que se $\tau: M \rightarrow M$ é o gerador de uma ação livre $\varrho: \mathbb{Z}_{p} \times M \rightarrow M$, então $\tau(x) \neq x$ para todo $x \in M$ e $\tau^{p}=I d_{M}$. Vale uma recíproca, no sentido do seguinte resultado:

Lema 6.2.2. Seja $\tau: M \rightarrow M$ uma função tal que $\tau(x) \neq x$ para todo $x \in M$ e $\tau^{p}=I d_{M}$. Então, $\tau$ define uma ação livre de $\mathbb{Z}_{p}$ em $M$ do seguinte modo:

$$
\begin{aligned}
\varrho: \mathbb{Z}_{p} \times M & \longrightarrow M \\
(\bar{k}, x) & \longmapsto \varrho(\bar{k}, x)=\tau^{k}(x) .
\end{aligned}
$$

Mais ainda, $\tau$ é o gerador de $\varrho$.

Demonstração. Primeiramente notemos que $\varrho$ é bem definida. De fato, se $k_{1} \equiv k_{2} \bmod p$, então existe $m \in \mathbb{Z}$ tal que $k_{1}=k_{2}+m p$. Assim, para cada $x \in M$, temos que

$$
\tau^{k_{1}}(x)=\tau^{k_{2}+m p}(x)=\tau^{k_{2}}\left(\tau^{m p}(x)\right)=\tau^{k_{2}}(\overbrace{\left(\tau^{p} \circ \cdots \circ \tau^{p}\right)}^{m}(x))=\tau^{k_{2}}(x)
$$

e portanto, $\varrho\left(\overline{k_{1}}, x\right)=\varrho\left(\overline{k_{2}}, x\right)$. É fácil ver que $\varrho$ é uma ação. Mostremos que de fato esta ação é livre. Para isto, notemos que é suficiente mostrar que para cada $x \in M$, o conjunto

$$
\left\{x, \tau(x), \ldots, \tau^{p-1}(x)\right\}
$$


tem $p$ elementos distintos. Suponhamos primeiramente que $\tau^{a}(x)=x$ para algum $a \in\{1, \ldots, p-1\}$. Notemos que

$$
\tau^{m a}(x)=\left(\tau^{(m-1) a} \circ \tau^{a}\right)(x)=\tau^{(m-1) a}(x),
$$

e usando indução, segue que para todo $m \in\{1, \ldots, p-1\}$ vale $\tau^{m a}(x)=x$. Como $\mathbb{Z}_{p}$ é corpo, então $m a \equiv 1 \bmod p$ para algum $m \in\{1,2, \ldots, p-1\}$. Logo, existe $i \in \mathbb{Z}$ tal que $m a=1+i p$. Assim, temos que

$$
\tau(x)=(\overbrace{\tau^{p} \circ \cdots \circ \tau^{p}}^{i} \circ \tau)(x)=\left(\tau^{i p} \circ \tau\right)(x)=\tau^{i p+1}(x)=\tau^{m a}(x)=x
$$

o que é um absurdo. Segue que

$$
\tau^{a}(x) \neq x \text { para todo } x \in M \text { e para todo } a \in\{1,2, \ldots, p-1\} .
$$

Por fim, suponhamos que $\tau^{r}(x)=\tau^{s}(x)$ para algum $x \in M$, sendo $r, s \in\{0,1, \ldots, p-1\}$ e $r<s$. Então $\tau^{s-r}(x)=x$ e $s-r \in\{1, \ldots, p-1\}$, o que é um absurdo por (6.1). Isto mostra que para todo $x \in M$ o conjunto $\left\{x, \tau(x), \ldots, \tau^{p-1}(x)\right\}$ tem $p$ pontos distintos.

Notemos que pelo Lema 6.2.2, para definirmos uma ação livre de $\mathbb{Z}_{p}$ em $M$ é suficiente definir uma função $\tau: M \rightarrow M$ que satisfaz as hipóteses deste resultado. Por este motivo, a frase "seja $\mathbb{Z}_{p}$ uma ação livre em $M$ e $\tau: M \rightarrow M$ o gerador desta ação" significa que temos uma ação $\varrho: \mathbb{Z}_{p} \times M \rightarrow M$ e $\tau: M \rightarrow M$ é o gerador desta ação. Tal terminologia coincide com a escrita usual sobre ações livres de grupos (veja por exemplo [8]).

Feitas estas considerações, generalizamos a Definição 0.0.1 do seguinte modo:

Definição 6.2.3. Seja $\mathbb{Z}_{p}$ uma ação livre em $M$ e seja $\tau: M \rightarrow M$ o gerador desta ação. Dizemos que a tripla $\left(M, \mathbb{Z}_{p} ; N\right)$ tem a propriedade de Borsuk-Ulam se para cada função $f: M \rightarrow N$, existe $x \in M$ tal que o conjunto $\left\{f(x), f(\tau(x)), \ldots, f\left(\tau^{p-1}(x)\right)\right\}$ tem menos de $p$ elementos.

Fixemos $\tau: M \rightarrow M$ uma função que define uma ação livre de $\mathbb{Z}_{p}$ em $M$, conforme o Lema 6.2.2, sendo $M$ uma superfície fechada. Em $M$ definimos a seguinte relação:

$$
x \sim y \Leftrightarrow y \in\left\{x, \tau(x), \tau^{2}(x), \ldots, \tau^{p-1}(x)\right\} .
$$

Usando o fato que $\tau^{p}=I d_{M}$, é fácil mostrar que $\sim$ é uma relação de equivalência. Denotamos por $M_{\tau}$ o espaço quociente (também chamado espaço de órbitas) e por $p_{\tau}: M \rightarrow M_{\tau}$ a projeção natural. Como $M$ é uma superfície fechada, então $M_{\tau}$ também o é e $p_{\tau}$ é um recobrimento a $p$ folhas. Logo, temos a seguinte sequência exata (omitimos os pontos bases)

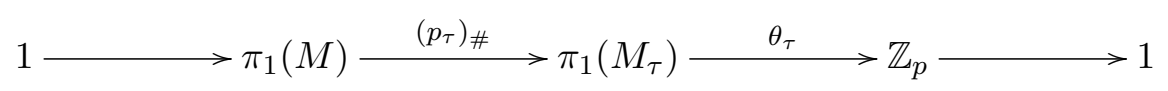

sendo que estamos identificando $\frac{\pi_{1}\left(M_{\tau}\right)}{\left(p_{\tau}\right)_{\#}\left(\pi_{1}(M)\right)} \operatorname{com} \mathbb{Z}_{p}$ e $\theta_{\tau}$ é a projeção natural.

Seja $N$ uma superfície fechada ou $\mathbb{R}^{2}$. Definimos

$$
F_{p}(N)=\left\{\left(x_{0}, x_{1}, \ldots, x_{p-1}\right) \in N \times \cdots \times N \mid x_{i} \neq x_{j} \text { se } i \neq j\right\},
$$

o qual é chamado de $p$-espaço de configuração ordenado de $N$.

Definimos $\tau_{1}: F_{p}(N) \rightarrow F_{p}(N)$ pela fórmula

$$
\tau_{1}\left(x_{0}, x_{1}, \ldots, x_{p-1}\right)=\left(x_{1}, \ldots, x_{p-1}, x_{0}\right) .
$$

É fácil ver que $\tau_{1}$ é o gerador de uma ação livre de $\mathbb{Z}_{p}$, conforme a Definição 6.2.1 e o Lema 6.2.2. Denotamos por $Q_{p}(N)$ o espaço de órbitas desta ação e por $j: F_{p}(N) \rightarrow Q_{p}(N)$ a projeção natural. Notemos que $j$ é um recobrimento a $p$ folhas. 
O grupo $\pi_{1}\left(F_{p}(N)\right)$ é denotado por $P_{p}(N)$ (não estamos nos preocupando com ponto base) e é chamado de grupo de $p$-tranças puras de $N$. Denotamos o grupo $\pi_{1}\left(Q_{p}(N)\right)$ por $G_{p}(N)$. Obtemos assim, a seguinte sequência exata

$$
1 \longrightarrow P_{p}(N) \stackrel{j_{\#}}{\longrightarrow} G_{p}(N) \stackrel{\pi}{\longrightarrow} \mathbb{Z}_{p} \longrightarrow 1
$$

sendo que estamos identificando $\frac{G_{p}(N)}{j_{\#}\left(P_{p}(N)\right)} \operatorname{com} \mathbb{Z}_{p}$ e $\pi$ é a projeção natural.

Agora, vamos fazer uma construção totalmente análoga ao que fizemos na Seção 1.4.

Suponhamos que $\left(M, \mathbb{Z}_{p} ; N\right)$ não tem a propriedade de Borsuk-Ulam. Por definição, existe uma função $f: M \rightarrow N$ tal que para todo $x \in M$ o conjunto $\left\{f(x), f(\tau(x)), \ldots, f\left(\tau^{p-1}(x)\right)\right\}$ tem $p$ pontos distintos. Logo, está bem definida a seguinte função:

$$
\begin{aligned}
F: M & \longrightarrow F_{p}(N) \\
x & \longmapsto\left(f(x), f(\tau(x)), \ldots, f\left(\tau^{p-1}(x)\right)\right) .
\end{aligned}
$$

Para todo $x \in M$ temos

$$
F(\tau(x))=\left(f(\tau(x)), f(\tau(\tau(x))), \ldots, f\left(\tau\left(\tau^{p-1}(x)\right)\right)\right)=(f(\tau(x)), f(\tau(\tau(x))), \ldots, f(x))=\tau_{1}(F(x))
$$

donde segue $F\left(\tau^{k}(x)\right)=\tau_{1}^{k}(F(x))$ para todo $k \in\{0,1, \ldots, p-1\}$. Logo, $F$ se passa ao quociente, isto é, existe $\bar{F}: M_{\tau} \rightarrow Q_{p}(N)$ tal que o seguinte diagrama é comutativo:

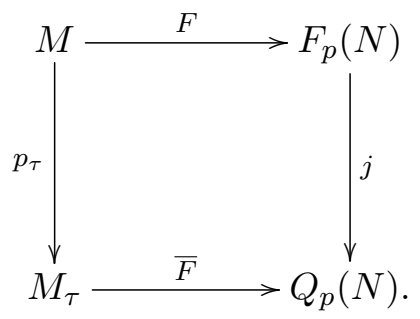

Aplicando o funtor $\pi_{1}$ neste diagrama e usando argumentos análogos aos que usamos na demonstração do Lema 1.4.1, obtemos o seguinte diagrama comutativo:

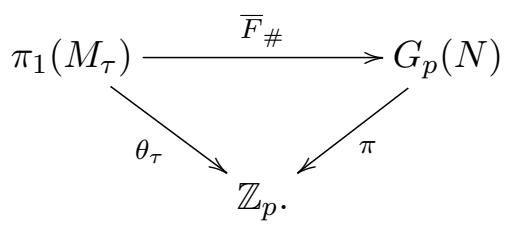

Nos casos em que $N \neq \mathbb{S}^{2}, \mathbb{R P}^{2}$, vale a recíproca. Mais precisamente, temos o seguinte resultado:

Lema 6.2.4. Sejam uma ação livre de $\mathbb{Z}_{p}$ em $M$, sendo $M$ uma superfície fechada, $\tau: M \rightarrow M$ o gerador desta ação e $N$ igual ao plano $\mathbb{R}^{2}$ ou igual a uma superfície fechada diferente de $\mathbb{S}^{2}$ ou $\mathbb{R}^{2}$. A tripla $\left(M, \mathbb{Z}_{p} ; N\right)$ não tem a propriedade de Borsuk-Ulam se, e somente se, existe um homomorfismo $\psi: \pi_{1}\left(M_{\tau}\right) \rightarrow G_{p}(N)$ tal que é comutativo o seguinte diagrama:

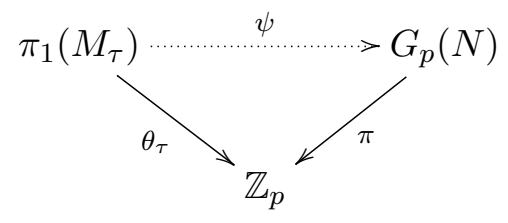

sendo $\theta_{\tau}$ como definido em (6.2) e $\pi$ como definido em (6.3).

Demonstração. A parte "somente se" já está feita. Basta tomar $\psi=\bar{F}_{\#}$, sendo $\bar{F}: M_{\tau} \rightarrow Q_{p}(N)$ 
como construída anteriormente.

Suponhamos então que temos o diagrama comutativo (6.4). Se $x \in \pi_{1}\left(M_{\tau}\right)$ é tal que $\theta_{\tau}(x)=\overline{0}$, então $(\pi \circ \psi)(x)=\theta_{\tau}(x)=\overline{0}$. Logo, por restrição, está bem definido o homomorfismo $\left.\psi\right|_{\operatorname{ker} \theta_{\tau}}: \operatorname{ker} \theta_{\tau} \rightarrow \operatorname{ker} \pi$. Pela exatidão das sequências (6.2) e (6.3), existe um homomorfismo $\varphi: \pi_{1}(M) \rightarrow P_{p}(M)$ tal que é comutativo o seguinte diagrama:

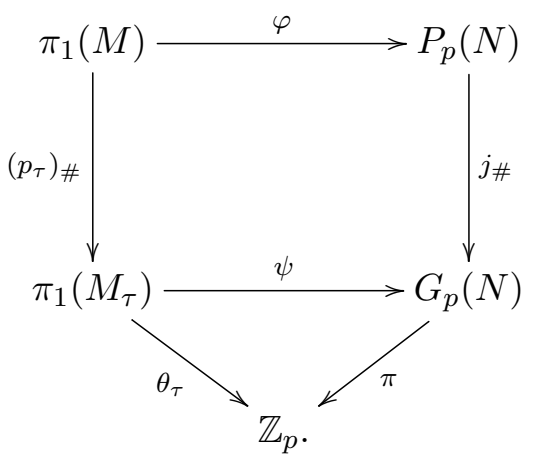

Como $N$ é igual ao $\mathbb{R}^{2}$ ou é igual a uma superfície fechada diferente de $\mathbb{S}^{2}$ ou $\mathbb{R P}^{2}$, em [13, Corolário 2.2] está demonstrado que $\pi_{i}\left(F_{p}(N)\right)$ é trivial se $i>1$. Como $j: F_{p}(N) \rightarrow Q_{p}(N)$ é um recobrimento, segue $\pi_{i}\left(Q_{p}(N)\right)$ é trivial se $i>1$. A partir daqui, a argumentação segue de maneira totalmente análoga as demonstrações do Lema 1.4.1 e do Lema Fundamental 1.4.3 e por isso vamos omitir o restante da prova.

O objetivo do restante deste capítulo é provar o seguinte resultado:

Teorema 6.2.5. Sejam $\mathbb{Z}_{p}$ uma ação livre em $M$, sendo $p>2$ e $M$ uma superfície fechada, $e$ $\tau: M \rightarrow M$ o gerador desta ação. Seja ainda $N$ igual ao plano $\mathbb{R}^{2}$ ou igual a uma superfície fechada. Existe uma função $f: M \rightarrow N$ que é homotópica a uma função constante tal que para todo $x \in M$ o conjunto $\left\{x, \tau(x), \ldots, \tau^{p-1}(x)\right\}$ tem $p$ elementos distintos, ou equivalentemente, a imagem da órbita de $x$ pela função $f$ tem $p$ pontos distintos. Portanto, $\left(M, \mathbb{Z}_{p} ; N\right)$ não tem a propriedade de Borsuk-Ulam.

Antes de provar o Teorema 6.2.5, vamos fazer algumas considerações sobre o espaço de órbitas $M_{\tau}$ e seu grupo fundamental.

Se $M_{\tau}$ é orientável, então $\pi_{1}\left(M_{\tau}\right)$ tem a seguinte presentação:

$$
\pi_{1}\left(M_{\tau}\right)=\left\langle a_{1}, a_{2}, \ldots a_{2 n-1}, a_{2 n} \mid\left[a_{1}, a_{2}\right] \cdots\left[a_{2 n-1}, a_{2 n}\right]\right\rangle .
$$

Se $M_{\tau}$ é não orientável, dependendo da característica de Euler de $M_{\tau}$, nós temos as seguintes possibilidades para presentação de $\pi_{1}\left(M_{\tau}\right)$ :

$$
\begin{aligned}
& \pi_{1}\left(M_{\tau}\right)=\left\langle u, v, a_{1}, a_{2}, a_{2 n-1}, a_{2 n} \mid u v u v^{-1}\left[a_{1}, a_{2}\right] \cdots\left[a_{2 n-1}, a_{2 n}\right]\right\rangle, \text { se } \chi\left(M_{\tau}\right) \text { é par; } \\
& \pi_{1}\left(M_{\tau}\right)=\left\langle c, a_{1}, a_{2}, a_{2 n-1}, a_{2 n} \mid c^{2}\left[a_{1}, a_{2}\right] \cdots\left[a_{2 n-1}, a_{2 n}\right]\right\rangle, \text { se } \chi\left(M_{\tau}\right) \text { é ímpar. }
\end{aligned}
$$

Lema 6.2.6. Sejam $\mathbb{Z}_{p}$ uma ação livre em $M$, sendo $M$ uma superfície fechada, e $\tau: M \rightarrow M$ um gerador desta ação. Suponhamos que $p \neq 2$ e o espaço de órbitas $M_{\tau}$ é não orientável. Valem as seguintes condiçôes, sendo $\theta_{\tau}: \pi_{1}\left(M_{\tau}\right) \rightarrow \mathbb{Z}_{p}$ como definido em (6.2):

- Se $\chi\left(M_{\tau}\right)$ é par, então $\theta_{\tau}(u)=\overline{0}$.

- Se $\chi\left(M_{\tau}\right)$ é impar, então $\theta_{\tau}(c)=\overline{0}$. 
Demonstração. Suponhamos que $\chi\left(M_{\tau}\right)$ é par. Como $\theta_{\tau}$ é homomorfismo, então

$$
\theta_{\tau}\left(u v u v^{-1}\left[a_{1}, a_{2}\right] \ldots\left[a_{2 n-1}, a_{2 n}\right]\right)=\overline{0} .
$$

Como $\mathbb{Z}_{p}$ é um grupo abeliano, a igualdade anterior se reduz a $2 \theta_{\tau}(u)=\overline{0}$. Portanto, o elemento $\theta_{\tau}(u)$ tem ordem par, mas como $p$ é um inteiro primo ímpar, não existem elementos não nulos de ordem par. Segue que $\theta_{\tau}(u)=\overline{0}$. A prova é análoga para o caso em que $\chi\left(M_{\tau}\right)$ é ímpar, e por isso a omitimos.

Demonstração do Teorema 6.2.5. Primeiramente, vamos mostrar que $\left(M, \mathbb{Z}_{p} ; \mathbb{R}^{2}\right)$ não tem a propriedade de Borsuk-Ulam. Como a sequência (6.3) é exata, então $\pi: G_{p}\left(\mathbb{R}^{2}\right) \rightarrow \mathbb{Z}_{p}$ é sobrejetora. Logo, existe

$$
b \in G_{p}\left(\mathbb{R}^{2}\right) \text { tal que } \pi(b)=\overline{1} .
$$

$1^{o}$ caso: Suponhamos que $M_{\tau}$ é orientável. Para cada gerador $a_{i} \in \pi_{1}\left(M_{\tau}\right)$, seja

$$
k_{i} \in\{0,1,2, \ldots, p-1\} \text { tal que } \theta_{\tau}\left(a_{i}\right)=\overline{k_{i}} .
$$

Definimos

$$
\begin{aligned}
\mu:\left\{a_{1}, \ldots, a_{n}\right\} & \longrightarrow G_{p}\left(\mathbb{R}^{2}\right) \\
a_{i} & \longmapsto \mu\left(a_{i}\right)=b^{k_{i}} .
\end{aligned}
$$

Notemos que

$$
\mu\left(\left[a_{1}, a_{2}\right] \cdots\left[a_{2 n-1}, a_{2 n}\right]\right)=\left[b^{k_{1}}, b^{k_{2}}\right] \cdots\left[b^{k_{2 n-1}}, b^{k_{2 n}}\right]=1,
$$

e portanto, nós temos um bem definido homomorfismo $\psi: \pi_{1}\left(M_{\tau}\right) \rightarrow G_{p}\left(\mathbb{R}^{2}\right)$, o qual no conjunto de geradores coincide com $\mu$. Mostremos que temos uma diagrama comutativo como em (6.4). Para todo $i \in\{1, \ldots, n\}$, temos

$$
(\pi \circ \psi)\left(a_{i}\right)=\pi\left(\psi\left(a_{i}\right)\right)=\pi\left(\mu\left(a_{i}\right)\right)=\pi\left(b^{k_{i}}\right)=k_{i}(\pi(b))=k_{i} \overline{1}=\overline{k_{i}}=\theta_{\tau}\left(a_{i}\right) .
$$

Segue pelo Lema 6.2.4 que $\left(M, \mathbb{Z}_{p} ; \mathbb{R}^{2}\right)$ não tem a propriedade de Borsuk-Ulam.

$2^{\circ}$ caso: Suponhamos que $M_{\tau}$ é não orientável e $\chi\left(M_{\tau}\right)$ é par. Seja

$$
k_{v} \in\{0,1,2, \ldots, p-1\} \text { tal que } \theta_{\tau}(v)=\overline{k_{v}} .
$$

Definimos

$$
\begin{aligned}
\nu:\left\{u, v, a_{1}, \ldots, a_{n}\right\} & \longrightarrow G_{p}\left(\mathbb{R}^{2}\right) \\
u & \longmapsto \nu(u)=1 \\
v & \longmapsto \nu(v)=b^{k_{v}} \\
a_{i} & \longmapsto \nu\left(a_{i}\right)=\mu\left(a_{i}\right) .
\end{aligned}
$$

sendo $\mu\left(a_{i}\right)$ como definido em (6.5). Notemos que

$$
\nu\left(u v u v^{-1}\left[a_{1}, a_{2}\right] \cdots\left[a_{2 n-1}, a_{2 n}\right]\right)=1 b^{k_{v}} 1\left(b^{k_{v}}\right)^{-1}\left[b^{k_{1}}, b^{k_{2}}\right] \cdots\left[b^{k_{2 n-1}}, b^{k_{2 n}}\right]=1,
$$

e portanto, temos um bem definido homomorfismo $\psi: \pi_{1}\left(M_{\tau}\right) \rightarrow G_{p}\left(\mathbb{R}^{2}\right)$, o qual coincide com $\nu$ no conjunto de geradores. Vamos mostrar que com esta definição, temos uma diagrama comutativo como em (6.4). De maneira análoga ao que fizemos no $1^{o}$ caso, temos que $(\pi \circ \psi)\left(a_{i}\right)=\theta_{\tau}\left(a_{i}\right)$, para todo $i \in\{1, \ldots, n\}$, e $(\pi \circ \psi)(v)=\theta_{\tau}(v)$. Pelo Lema 6.2.6, nós temos que $\theta_{\tau}(u)=\overline{0}$, e portanto temos que

$$
(\pi \circ \psi)(u)=\pi(\psi(u))=\pi(\nu(u))=\pi(1)=\overline{0}=\theta_{\tau}(u) .
$$

Segue pelo Lema 6.2.4 que $\left(M, \mathbb{Z}_{p} ; \mathbb{R}^{2}\right)$ não tem a propriedade de Borsuk-Ulam.

$3^{\circ}$ caso: Suponhamos que $M_{\tau}$ é não orientável e $\chi\left(M_{\tau}\right)$ é ímpar. Vamos omitir a demonstração deste caso, pois é totalmente análoga ao caso anterior. 
Como $\left(M, \mathbb{Z}_{p} ; \mathbb{R}^{2}\right)$ não tem a propriedade de Borsuk-Ulam, por definição, existe uma função $g: M \rightarrow \mathbb{R}^{2}$ tal que para todo $x \in M$ o conjunto $\left\{g(x), g(\tau(x)), \ldots, g\left(\tau^{p-1}(x)\right)\right\}$ tem $p$ pontos distintos. Como $N$ é uma superfície fechada ou $\mathbb{R}^{2}$, então existe um mergulho $i: \mathbb{R}^{2} \rightarrow N$. Logo, a função $f: M \rightarrow N$ definida por $f(x)=(i \circ g)(x)$ para todo $x \in M$ é homotópica a uma função constante, uma vez que $\mathbb{R}^{2}$ é contrátil, e tal que o conjunto

$$
\left\{f(x), f(\tau(x)), \ldots, f\left(\tau^{p-1}(x)\right)\right\}
$$

tem $p$ pontos distintos para todo $x \in M$. Portanto, a tripla $\left(M, \mathbb{Z}_{p} ; N\right)$ não tem a propriedade de Borsuk-Ulam.

cabeçalho para os apêndices 


\section{Apêndice A}

\section{Os grupos de tranças do Toro}

O objetivo deste apêndice é demonstrar que $P_{2}\left(\mathbb{T}^{2}\right)$ é isomorfo a $F(x, y) \oplus \mathbb{Z} \oplus \mathbb{Z}$ e mostrar como um elemento especial de $B_{2}\left(\mathbb{T}^{2}\right)$, chamado $\sigma$, atua por conjugação nesta apresentação. Este mesmo resultado poderá ser encontrado em [17].

Nós usaremos neste apêndice o fato que $\mathbb{T}^{2}$ menos uma 2-célula é homeomorfo a um disco $\mathbb{D}^{2}$ unido com 2 alças não torcidas. Tal fato está enunciado, por exemplo, em [25] e pode ser visualizado através da seguinte sequência de figuras:

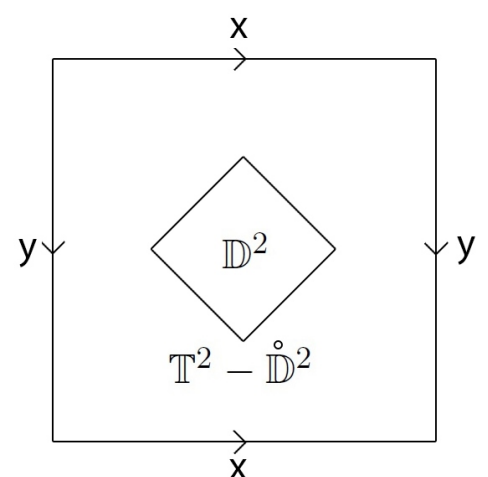

Figura A.1: Retirando um disco do Toro.

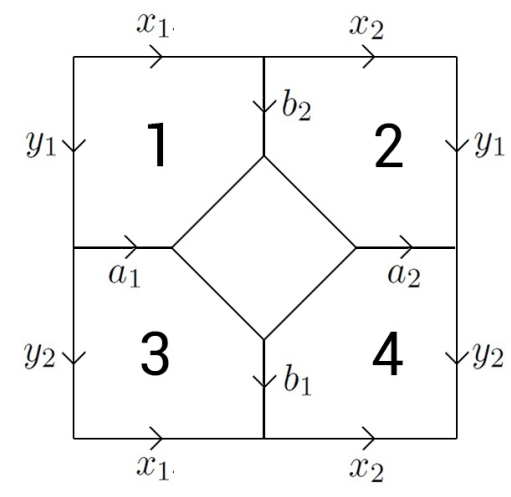

Figura A.2: "Retalhando".

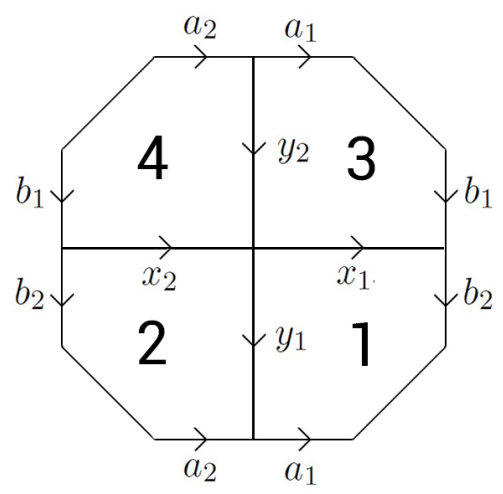

Figura A.3: "Remontando".

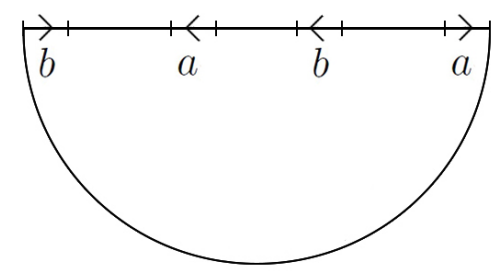

Figura A.4: "Arredondando" a figura A.3, sendo $a=a_{1} a_{2}$ $e b=b_{1} b_{2}$.

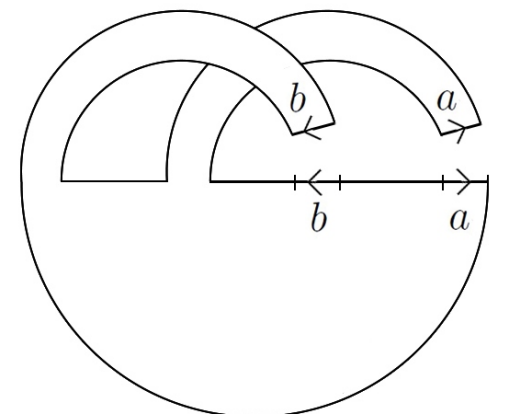

Figura A.5: "Colocando as alças".

A partir de [12, Seção 4], nós temos o seguinte resultado: 
Proposição A.0.7. $P_{2}\left(\mathbb{T}^{2}\right)$ admite a seguinte apresentação:

Geradores: $B, \rho_{1,1}, \rho_{1,2}, \rho_{2,1}, \rho_{2,2}$

Relaçôes:

(i) $\left[\rho_{1,1}, \rho_{1,2}^{-1}\right]=\left[\rho_{2,1}, \rho_{2,2}^{-1}\right]=B$;

(ii) $\rho_{2, k} \rho_{1, k} \rho_{2, k}^{-1}=B \rho_{1, k} B^{-1}$ e $\rho_{2, k}^{-1} \rho_{1, k} \rho_{2, k}=\rho_{1, k}\left[B^{-1}, \rho_{1, k}\right], k \in\{1,2\}$;

(iii) $\rho_{2,1} \rho_{1,2} \rho_{2,1}^{-1}=B \rho_{1,2}\left[\rho_{1,1}^{-1}, B\right]$ e $\rho_{2,1}^{-1} \rho_{1,2} \rho_{2,1}=B^{-1}\left[B, \rho_{1,1}\right] \rho_{1,2}\left[B^{-1}, \rho_{1,1}\right]$;

(iv) $\rho_{2,2} \rho_{1,1} \rho_{2,2}^{-1}=\rho_{1,1} B^{-1}$ e $\rho_{2,2}^{-1} \rho_{1,1} \rho_{2,2}=\rho_{1,1} B\left[B^{-1}, \rho_{1,2}\right]$.

A representação geométrica dos geradores é dada pelas figuras A.6, A.7, A.8, A.9 e A.10:

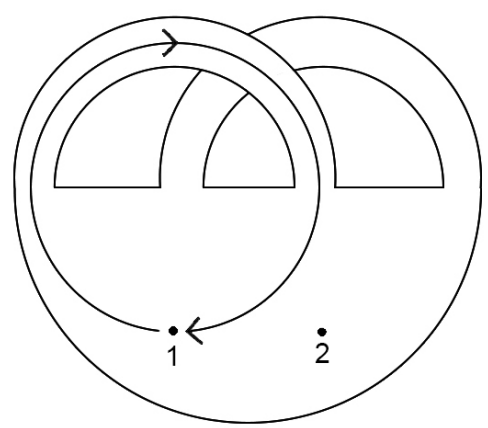

Figura A.6: gerador $\rho_{1,1}$

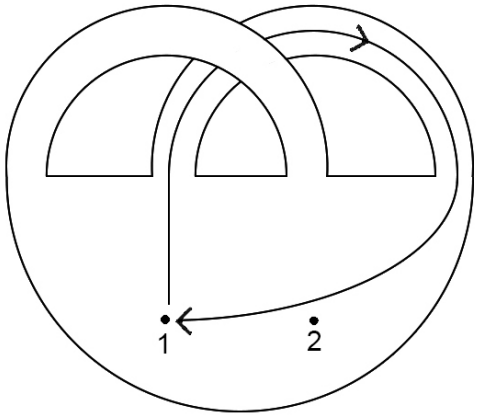

Figura A.7: gerador $\rho_{1,2}$.

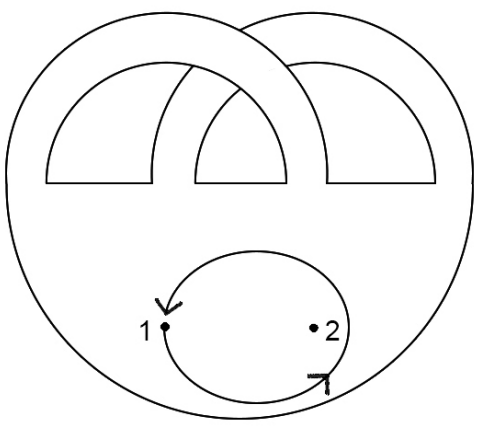

Figura A.8: gerador $B$.

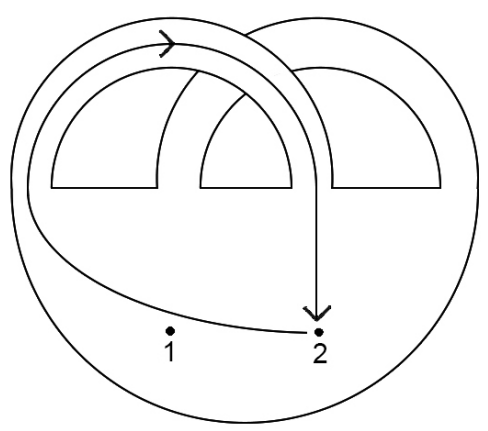

Figura A.9: gerador $\rho_{2,1}$

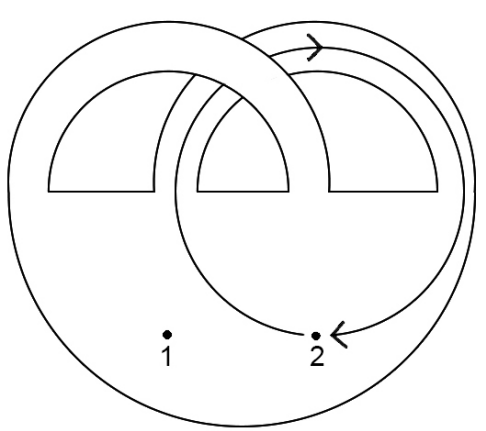

Figura A.10: gerador $\rho_{2,2}$.

Notemos que as relações descritas na Proposição A.0.7 são em termos da ação de $\rho_{2,1}$ e $\rho_{2,2}$ por conjugação nos elementos $\rho_{1,1}$ e $\rho_{1,2}$. Para facilitar certos cálculos, vamos mostrar no próximo resultado como $\rho_{2,1}$ e $\rho_{2,2}$ atuam em $B$.

Lema A.0.8. Em $P_{2}\left(\mathbb{T}^{2}\right)$ valem as seguintes relaçôes:
(a) $\rho_{2,1} B \rho_{2,1}^{-1}=B \rho_{1,1}^{-1} B \rho_{1,1} B^{-1}$;
(b) $\rho_{2,2} B \rho_{2,2}^{-1}=B \rho_{1,2}^{-1} B \rho_{1,2} B^{-1}$.

Demonstração. Vamos usar a presentação de $P_{2}\left(\mathbb{T}^{2}\right)$ para mostrar que valem as fórmulas $(a)$ e $(b)$. Temos que

$$
\begin{array}{rll}
\rho_{2,1} B \rho_{2,1}^{-1} & \stackrel{(i)}{=} & {\left[\rho_{2,1} \rho_{1,1} \rho_{2,1}^{-1}, \rho_{2,1} \rho_{1,2}^{-1} \rho_{2,1}^{-1}\right]} \\
& \stackrel{(i i),(i i i)}{=} & B \rho_{1,1} B^{-1}\left[B, \rho_{1,1}^{-1}\right] \rho_{1,2}^{-1} B^{-1} B \rho_{1,1}^{-1} B^{-1} B \rho_{1,2}\left[\rho_{1,1}^{-1}, B\right] \\
& =B \rho_{1,1} B^{-1} B \rho_{1,1}^{-1} B^{-1} \rho_{1,1} \rho_{1,2}^{-1} \rho_{1,1}^{-1} B^{-1} B \rho_{1,2} \rho_{1,1}^{-1} B \rho_{1,1} B^{-1} \\
& \stackrel{(i)}{=} & B \rho_{1,1}^{-1} B \rho_{1,1} B^{-1}
\end{array}
$$


e portanto, vale $(a)$. Agora, temos que

$$
\begin{array}{rll}
\rho_{2,2} B \rho_{2,2}^{-1} & \stackrel{(i)}{=} & {\left[\rho_{2,2} \rho_{1,1} \rho_{2,2}^{-1}, \rho_{2,2} \rho_{1,2}^{-1} \rho_{2,2}^{-1}\right]} \\
& \stackrel{(i i),(i v)}{=} & \rho_{1,1} B^{-1} B \rho_{1,2}^{-1} B^{-1} B \rho_{1,1}^{-1} B \rho_{1,2} B^{-1} \\
= & \rho_{1,1} \rho_{1,2}^{-1} \rho_{1,1}^{-1}\left(\rho_{1,2} \rho_{1,2}^{-1}\right) B \rho_{1,2} B^{-1} \\
& \stackrel{(i)}{=} & B \rho_{1,2}^{-1} B \rho_{1,2} B^{-1}
\end{array}
$$

e logo, vale (b).

No próximo resultado, vamos exibir dois elementos que pertencem ao centro de $P_{2}\left(\mathbb{T}^{2}\right)$. No decorrer do texto, ficará claro que estes elemento na verdade geram todo o centro do grupo de tranças do Toro.

Proposição A.0.9. Os elementos $\rho_{1,1} B^{-1} \rho_{2,1}$ e $\rho_{1,2} B^{-1} \rho_{2,2}$ pertencem ao centro de $P_{2}\left(\mathbb{T}^{2}\right)$.

Demonstração. Vamos usar as relações dadas na Proposição A.0.7 e no Lema A.0.8 para mostrar que os elementos $\rho_{1,1} B^{-1} \rho_{2,1}$ e $\rho_{1,2} B^{-1} \rho_{2,2}$ comutam com os geradores de $P_{2}\left(\mathbb{T}^{2}\right)$, o que é suficiente para que o resultado fique provado. Primeiramente, temos que

$$
\begin{gathered}
{\left[\rho_{1,1} B^{-1} \rho_{2,1}, \rho_{1,1}\right]=\rho_{1,1} B^{-1}\left(\rho_{2,1} \rho_{1,1} \rho_{2,1}^{-1}\right) B \rho_{1,1}^{-1} \rho_{1,1}^{-1}=\rho_{1,1} B^{-1} B \rho_{1,1} B^{-1} B \rho_{1,1}^{-1} \rho_{1,1}^{-1}=1,} \\
{\left[\rho_{1,1} B^{-1} \rho_{2,1}, \rho_{1,2}\right]=\rho_{1,1} B^{-1}\left(\rho_{2,1} \rho_{1,2} \rho_{2,1}^{-1}\right) B \rho_{1,1}^{-1} \rho_{1,2}^{-1}=\rho_{1,1} B^{-1} B \rho_{1,2}\left[\rho_{1,1}^{-1}, B\right] B \rho_{1,1}^{-1} \rho_{1,2}^{-1}} \\
=\rho_{1,1} \rho_{1,2} \rho_{1,1}^{-1} B \rho_{1,1} B^{-1} B \rho_{1,1}^{-1} \rho_{1,2}^{-1}=\rho_{1,1} \rho_{1,2} \rho_{1,1}^{-1} \rho_{1,1} \rho_{1,2}^{-1} \rho_{1,1}^{-1} \rho_{1,2} \rho_{1,1} \rho_{1,1}^{-1} \rho_{1,2}^{-1}=1, \\
{\left[\rho_{1,1} B^{-1} \rho_{2,1}, \rho_{2,1}\right]} \\
=\rho_{1,1} B^{-1} \rho_{2,1} \rho_{2,1} \rho_{2,1}^{-1} B \rho_{1,1}^{-1} \rho_{2,1}^{-1}=\rho_{1,1} B^{-1}\left(\rho_{2,1} B \rho_{2,1}^{-1}\right)\left(\rho_{2,1} \rho_{1,1} \rho_{2,1}^{-1}\right)^{-1} \\
=\rho_{1,1} B^{-1} B \rho_{1,1}^{-1} B \rho_{1,1} B^{-1} B \rho_{1,1}^{-1} B^{-1}=1
\end{gathered}
$$

e

$$
\begin{aligned}
{\left[\rho_{1,1} B^{-1} \rho_{2,1}, \rho_{2,2}\right] } & =\rho_{1,1} B^{-1} \rho_{2,1} \rho_{2,2} \rho_{2,1}^{-1} B \rho_{1,1}^{-1} \rho_{2,2}^{-1}=\rho_{1,1} B^{-1}\left(\rho_{2,2} \rho_{2,2}^{-1}\right) \rho_{2,1} \rho_{2,2} \rho_{2,1}^{-1} B \rho_{1,1}^{-1} \rho_{2,2}^{-1} \\
& =\rho_{1,1} B^{-1} \rho_{2,2} B^{-1} B \rho_{1,1}^{-1} \rho_{2,2}^{-1}=\rho_{1,1} B^{-1}\left(\rho_{2,2} \rho_{1,1}^{-1} \rho_{2,2}^{-1}\right)=\rho_{1,1} B^{-1} B \rho_{1,1}=1
\end{aligned}
$$

Agora, temos que

$$
\begin{gathered}
{\left[\rho_{1,2} B^{-1} \rho_{2,2}, \rho_{1,1}\right]=\rho_{1,2} B^{-1}\left(\rho_{2,2} \rho_{1,1} \rho_{2,2}^{-1}\right) B \rho_{1,2}^{-1} \rho_{1,1}^{-1}=\rho_{1,2} B^{-1} \rho_{1,1} B^{-1} B \rho_{1,2}^{-1} \rho_{1,1}^{-1}} \\
=\rho_{1,2} \rho_{1,2}^{-1} \rho_{1,1} \rho_{1,2} \rho_{1,1}^{-1} \rho_{1,1} \rho_{1,2}^{-1} \rho_{1,1}^{-1}=1, \\
{\left[\rho_{1,2} B^{-1} \rho_{2,2}, \rho_{1,2}\right]=\rho_{1,2} B^{-1}\left(\rho_{2,2} \rho_{1,2} \rho_{2,2}^{-1}\right) B \rho_{1,2}^{-1} \rho_{1,2}^{-1}=\rho_{1,2} B^{-1} B \rho_{1,2} B^{-1} B \rho_{1,2}^{-1} \rho_{1,2}^{-1}=1,} \\
{\left[\rho_{1,2} B^{-1} \rho_{2,2}, \rho_{2,1}\right]=\rho_{1,2} B^{-1} \rho_{2,2} \rho_{2,1} \rho_{2,2}^{-1} B \rho_{1,2}^{-1} \rho_{2,1}^{-1}=\rho_{1,2} B^{-1} \rho_{2,2} \rho_{2,1} \rho_{2,2}^{-1}\left(\rho_{2,1}^{-1} \rho_{2,2} \rho_{2,2}^{-1} \rho_{2,1}\right) B \rho_{1,2}^{-1} \rho_{2,1}^{-1}} \\
=\rho_{1,2} B^{-1} \rho_{2,2} B \rho_{2,2}^{-1} \rho_{2,1} B \rho_{1,2}^{-1} \rho_{2,1}^{-1}=\rho_{1,2} B^{-1}\left(\rho_{2,2} B \rho_{2,2}^{-1}\right)\left(\rho_{2,1} B \rho_{2,1}^{-1}\right)\left(\rho_{2,1} \rho_{1,2}^{-1} \rho_{2,1}^{-1}\right) \\
=\rho_{1,2} B^{-1} B \rho_{1,2}^{-1} B \rho_{1,2} B^{-1} B \rho_{1,1}^{-1} B \rho_{1,1} B^{-1}\left[B, \rho_{1,1}^{-1}\right] \rho_{1,2}^{-1} B^{-1} \\
=B \rho_{1,2} \rho_{1,1}^{-1} B \rho_{1,1} B^{-1} B \rho_{1,1}^{-1} B^{-1} \rho_{1,1} \rho_{1,2}^{-1} B^{-1}=1
\end{gathered}
$$


$\mathrm{e}$

$$
\begin{aligned}
{\left[\rho_{1,2} B^{-1} \rho_{2,2}, \rho_{2,2}\right] } & =\rho_{1,2} B^{-1} \rho_{2,2} \rho_{2,2} \rho_{2,2}^{-1} B \rho_{1,2}^{-1} \rho_{2,2}^{-1}=\rho_{1,2} B^{-1}\left(\rho_{2,2} B \rho_{2,2}^{-1}\right)\left(\rho_{2,2} \rho_{1,2}^{-1} \rho_{2,2}^{-1}\right) \\
& =\rho_{1,2} B^{-1} B \rho_{1,2}^{-1} B \rho_{1,2} B^{-1} B \rho_{1,2}^{-1} B^{-1}=1 .
\end{aligned}
$$

Seja $f: P_{2}\left(\mathbb{T}^{2}\right) \rightarrow \mathbb{Z} \oplus \mathbb{Z}$ definida nos geradores por

$$
f\left(\rho_{1,1}\right)=(1,0) \quad f\left(\rho_{1,2}\right)=(0,1) \quad f\left(\rho_{2,1}\right)=f\left(\rho_{2,2}\right)=(0,0) .
$$

Usando a Proposição A.0.7, é fácil ver que de fato $f$ é um bem definido homomorfismo. Notemos que pelas figuras A.1, A.2, A.3, A.4 e A.5, $f$ é, a menos de isomorfismo, o homomorfismo induzido no grupo fundamental da aplicação $p_{1}: F_{2}\left(\mathbb{T}^{2}\right) \rightarrow \mathbb{T}^{2}$, definida por $p_{1}(x, y)=x$, e que geometricamente corresponde a eliminar a segunda corda de uma trança.

Notemos que pela Proposição A.0.9, está bem definido o homomorfismo $\varphi: \mathbb{Z} \oplus \mathbb{Z} \rightarrow P_{2}\left(\mathbb{T}^{2}\right)$ que nos geradores tem os seguintes valores:

$$
\varphi(1,0)=\rho_{1,1} B^{-1} \rho_{2,1} \quad \text { e } \quad \varphi(0,1)=\rho_{1,2} B^{-1} \rho_{2,2} .
$$

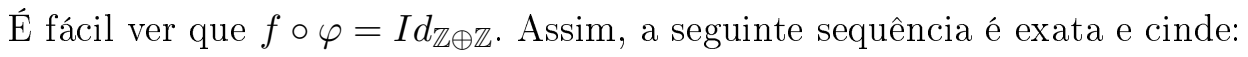

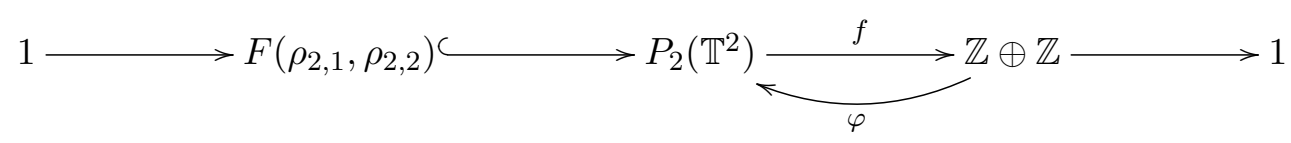

Lembremos que

$$
\begin{gathered}
F\left(\rho_{2,1}, \rho_{2,2}\right) \rtimes_{\theta}(\mathbb{Z} \oplus \mathbb{Z}) \stackrel{\lambda}{\underset{\gamma}{\rightleftarrows}} P_{2}\left(\mathbb{T}^{2}\right) \\
\lambda(b, c)=b \varphi(c) \quad \gamma(x)=\left(x \varphi\left(f\left(x^{-1}\right)\right), f(x)\right)
\end{gathered}
$$

são isomorfismos e $\lambda=\gamma^{-1}$, sendo $\theta: \mathbb{Z} \oplus \mathbb{Z} \longrightarrow \operatorname{Aut}\left(F\left(\rho_{2,1}, \rho_{2,2}\right)\right)$ definida do seguinte modo:

$$
\begin{aligned}
\theta(m, n): F\left(\rho_{2,1}, \rho_{2,2}\right) & \longrightarrow F\left(\rho_{2,1}, \rho_{2,2}\right) \\
x & \longmapsto \varphi(m, n) x \varphi(m, n)^{-1} .
\end{aligned}
$$

Novamente pela Proposição A.0.9, temos que o homomorfismo $\theta$ é trivial. Assim, nós temos os seguintes isomorfismos

$$
F\left(\rho_{2,1}, \rho_{2,2}\right) \oplus \mathbb{Z} \oplus \mathbb{Z} \underset{\gamma}{\stackrel{\lambda}{\rightleftarrows}} P_{2}\left(\mathbb{T}^{2}\right)
$$

Vamos calcular explicitamente $\lambda$ e $\gamma$ nos geradores. Afim de evitar confusões, nós denotaremos o elemento neutro de $F\left(\rho_{2,1}, \rho_{2,2}\right)$ pelo símbolo $\mathbb{1}$. Temos que

$$
\begin{aligned}
& \lambda\left(\rho_{2,1}, 0,0\right)=\rho_{2,1} \varphi(0,0)=\rho_{2,1}, \\
& \lambda\left(\rho_{2,2}, 0,0\right)=\rho_{2,2} \varphi(0,0)=\rho_{2,2}, \\
& \lambda(\mathbb{1}, 1,0)=\mathbb{1} \varphi(1,0)=\rho_{1,1} B^{-1} \rho_{2,1}, \\
& \lambda(\mathbb{1}, 0,1)=\mathbb{1} \varphi(0,1)=\rho_{1,2} B^{-1} \rho_{2,2},
\end{aligned}
$$




$$
\begin{aligned}
& \begin{aligned}
\gamma\left(\rho_{1,1}\right) & =\left(\rho_{1,1} \varphi\left(f\left(\rho_{1,1}^{-1}\right)\right), f\left(\rho_{1,1}\right)\right)=\left(\rho_{1,1} \varphi(1,0)^{-1}, 1,0\right)=\left(\rho_{1,1} \rho_{2,1}^{-1} B \rho_{1,1}^{-1}, 1,0\right) \\
& =\left(\rho_{2,1}^{-1} B \rho_{1,1}^{-1} \rho_{1,1}, 1,0\right)=\left(\rho_{2,1}^{-1} B, 1,0\right), \\
\gamma\left(\rho_{1,2}\right) & =\left(\rho_{1,2} \varphi\left(f\left(\rho_{1,2}^{-1}\right)\right), f\left(\rho_{1,2}\right)\right)=\left(\rho_{1,2} \varphi(0,1)^{-1}, 0,1\right)=\left(\rho_{1,2} \rho_{2,2}^{-1} B \rho_{1,2}^{-1}, 0,1\right) \\
& =\left(\rho_{2,2}^{-1} B \rho_{1,2}^{-1} \rho_{1,2}, 0,1\right)=\left(\rho_{2,2}^{-1} B, 0,1\right),
\end{aligned} \\
& \gamma\left(\rho_{2,1}\right)=\left(\rho_{2,1} \varphi\left(f\left(\rho_{2,1}^{-1}\right)\right), f\left(\rho_{2,1}\right)\right)=\left(\rho_{2,1} \varphi(0,0), 0,0\right)=\left(\rho_{1,1}, 0,0\right), \\
& \gamma\left(\rho_{2,2}\right)=\left(\rho_{2,2} \varphi\left(f\left(\rho_{2,2}^{-1}\right)\right), f\left(\rho_{2,2}\right)\right)=\left(\rho_{1,2} \varphi(0,0), 0,0\right)=\left(\rho_{1,2}, 0,0\right) . \\
& \text { Seja } \sigma \in B_{2}\left(\mathbb{T}^{2}\right) \text { definido geometricamente con- }
\end{aligned}
$$
forme a figura A.4.

Notemos que $\sigma^{2}=B$.

Seja agora $c_{\sigma}: P_{2}\left(\mathbb{T}^{2}\right) \rightarrow P_{2}\left(\mathbb{T}^{2}\right)$, definido por $c_{\sigma}(x)=\sigma x \sigma^{-1}$.

Geometricamente, é facil concluir que

$$
\begin{aligned}
& c_{\sigma}\left(\rho_{1,1}\right)=\rho_{2,1}, \\
& c_{\sigma}\left(\rho_{1,2}\right)=\rho_{2,2}, \\
& c_{\sigma}\left(\rho_{2,1}\right)=B \rho_{1,1} B^{-1}, \\
& c_{\sigma}\left(\rho_{2,2}\right)=B \rho_{1,2} B^{-1} .
\end{aligned}
$$

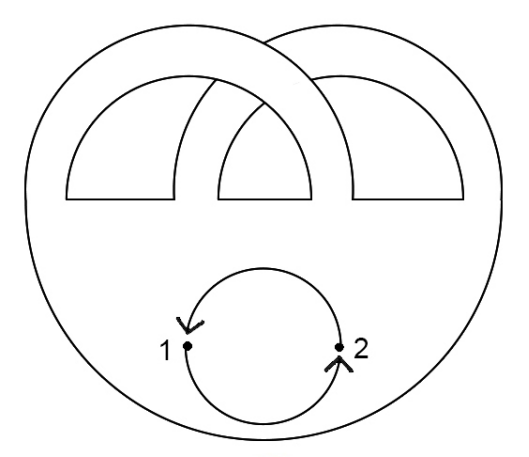

Figura A.11: $\sigma \in B_{2}\left(\mathbb{T}^{2}\right)$.

Definimos agora $l_{\sigma}: F\left(\rho_{2,1}, \rho_{2,2}\right) \oplus \mathbb{Z} \oplus \mathbb{Z} \rightarrow F\left(\rho_{2,1}, \rho_{2,2}\right) \oplus \mathbb{Z} \oplus \mathbb{Z}$ de modo que o seguinte diagrama seja comutativo

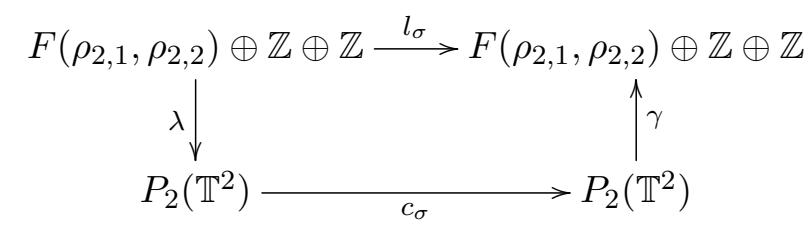

Vamos calcular $l_{\sigma}$ nos geradores de $F\left(\rho_{2,1}, \rho_{2,2}\right) \oplus \mathbb{Z} \oplus \mathbb{Z}$. Temos que

$$
\begin{aligned}
& \begin{aligned}
& l_{\sigma}\left(\rho_{2,1}, 0,0\right)=\left(\gamma \circ c_{\sigma} \gamma \lambda\right)\left(\rho_{2,1}, 0,0\right)=\left(\gamma \circ c_{\sigma}\right)\left(\rho_{2,1}\right)=\gamma\left(B \rho_{1,1} B^{-1}\right) \\
&=(B, 0,0)\left(\rho_{2,1}^{-1} B, 1,0\right)\left(B^{-1}, 0,0\right)=\left(B \rho_{2,1}^{-1}, 1,0\right), \\
& l_{\sigma}\left(\rho_{2,2}, 0,0\right)=\left(\gamma \circ c_{\sigma} \gamma \lambda\right)\left(\rho_{2,2}, 0,0\right)=\left(\gamma \circ c_{\sigma}\right)\left(\rho_{2,2}\right)=\gamma\left(B \rho_{1,2} B^{-1}\right) \\
&=(B, 0,0)\left(\rho_{2,2}^{-1} B, 0,1\right)\left(B^{-1}, 0,0\right)=\left(B \rho_{2,2}^{-1}, 0,1\right), \\
& l_{\sigma}(\mathbb{1}, 1,0)=\left(\gamma \circ c_{\sigma} \gamma \lambda\right)(\mathbb{1}, 1,0)=\left(\gamma \circ c_{\sigma}\right)\left(\rho_{1,1} B^{-1} \rho_{2,1}\right)=\gamma\left(\rho_{2,1} B^{-1} B \rho_{1,1} B^{-1}\right) \\
&=\gamma\left(\rho_{2,1} \rho_{1,1} B^{-1}\right)=\left(B \rho_{1,1}^{-1}, 1,0\right)\left(\rho_{1,1}, 0,0\right)\left(B^{-1}, 0,0\right)=(\mathbb{1}, 1,0)
\end{aligned}
\end{aligned}
$$


e

$$
\begin{aligned}
l_{\sigma}(\mathbb{1}, 0,1) & =\left(\gamma \circ c_{\sigma} \gamma \lambda\right)(\mathbb{1}, 0,1)=\left(\gamma \circ c_{\sigma}\right)\left(\rho_{1,2} B^{-1} \rho_{2,2}\right)=\gamma\left(\rho_{2,2} B^{-1} B \rho_{1,2} B^{-1}\right) \\
& =\gamma\left(\rho_{2,2} \rho_{1,2} B^{-1}\right)=\left(B \rho_{1,2}^{-1}, 0,1\right)\left(\rho_{1,2}, 0,0\right)\left(B^{-1}, 0,0\right)=(\mathbb{1}, 0,1) .
\end{aligned}
$$

Denotando $\rho_{2,1}$ por $x$ e $\rho_{2,2}$ por $y$, como resumo das informações obtidas, nós podemos fazer a seguinte observação:

Observação A.0.10. A menos de isomorfismo, $P_{2}\left(\mathbb{T}^{2}\right)$ se escreve na forma $F(x, y) \oplus \mathbb{Z} \oplus \mathbb{Z}$, sendo $F(x, y)$ o grupo livre gerado pelo conjunto $\{x, y\}$. Com respeito a esta escrita, temos que:

- $\left(p_{1}\right)_{\#}: P_{2}\left(\mathbb{T}^{2}\right) \rightarrow \pi_{1}\left(\mathbb{T}^{2}\right)=\mathbb{Z} \oplus \mathbb{Z}$ é a projeção de $F(x, y) \oplus \mathbb{Z} \oplus \mathbb{Z}$ em $\mathbb{Z} \oplus \mathbb{Z}$, isto é, $\left(p_{1}\right)_{\#}(w, m, n)=(m, n)$;

- Existe um elemento $\sigma \in B_{2}\left(\mathbb{T}^{2}\right)-P_{2}\left(\mathbb{T}^{2}\right)$ tal que $\sigma^{2}=(B, 0,0)$, sendo $B=\left[x, y^{-1}\right]$;

- O homomorfismo $l_{\sigma}: P_{2}\left(\mathbb{T}^{2}\right) \rightarrow P_{2}\left(\mathbb{T}^{2}\right)$, definido por $l_{\sigma}(b)=\sigma b \sigma^{-1}$ para todo $b \in P_{2}\left(\mathbb{T}^{2}\right)$, tem os seguintes valores nos geradores:

$$
\begin{array}{ll}
l_{\sigma}(x, 0,0)=\left(B x^{-1}, 1,0\right) & l_{\sigma}(\mathbb{1}, 1,0)=(\mathbb{1}, 1,0) \\
l_{\sigma}(y, 0,0)=\left(B y^{-1}, 0,1\right) & l_{\sigma}(\mathbb{1}, 0,1)=(\mathbb{1}, 0,1)
\end{array}
$$

sendo $\mathbb{1} \in F(x, y)$ o elemento neutro. 


\section{Apêndice B}

\section{Os grupos de tranças da garrafa de Klein}

O objetivo deste apêndice é demonstrar que $P_{2}\left(\mathbb{K}^{2}\right)$ é isomorfo a $F(u ; v) \rtimes_{\theta}(\mathbb{Z} \rtimes \mathbb{Z})$ para um homomorfismo $\theta: \mathbb{Z} \rtimes \mathbb{Z} \rightarrow \operatorname{Aut}(F(u, v))$ que será explicitado. Também para um elemento especial de $B_{2}\left(K^{2}\right)$, chamado de $\sigma$, calcularemos a acão de $\sigma$ em $P_{2}\left(K^{2}\right)$, onde esta acão será determinada usando o grupo $F(u ; v) \rtimes_{\theta}(\mathbb{Z} \rtimes \mathbb{Z})$ que foi determinado.

Nós usaremos neste apêndice o fato que $\mathbb{K}^{2}$ é homeomorfo a $\mathbb{R} \mathbb{P}^{2} \# \mathbb{R} \mathbb{P}^{2}$. Tal fato está enunciado, por exemplo, em [22, Capítulo 1, Exemplo 4.3] e pode ser visualizado através da seguinte sequência de figuras:

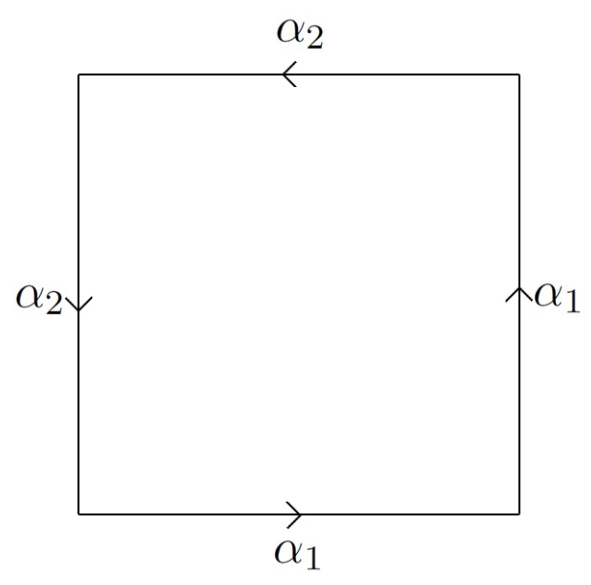

Figura B.1: $\mathbb{R} \mathbb{P}^{2} \# \mathbb{R} \mathbb{P}^{2}$.

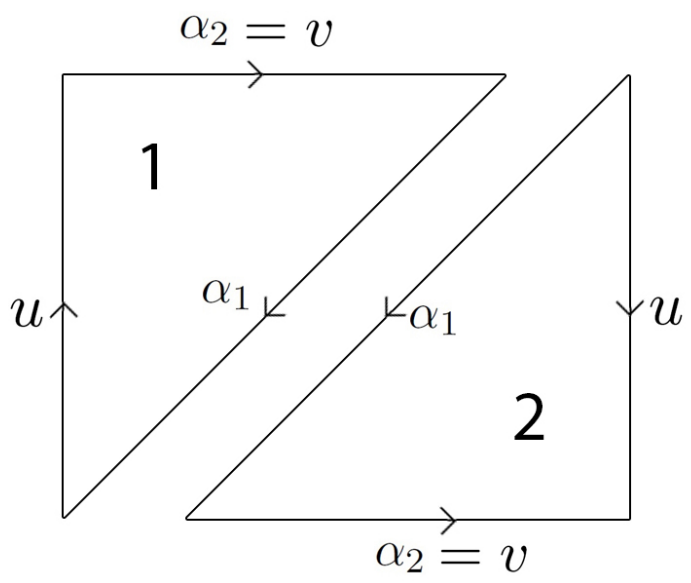

Figura B.3: "Rodando".

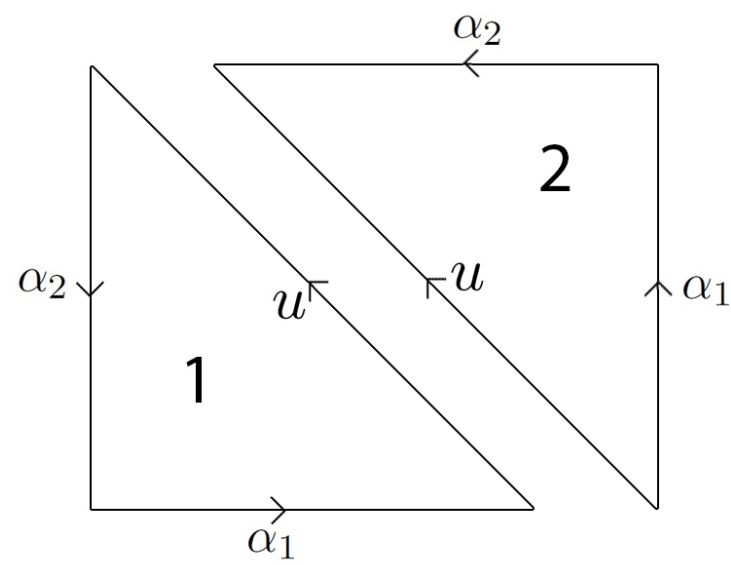

Figura B.2: "Retalhando".

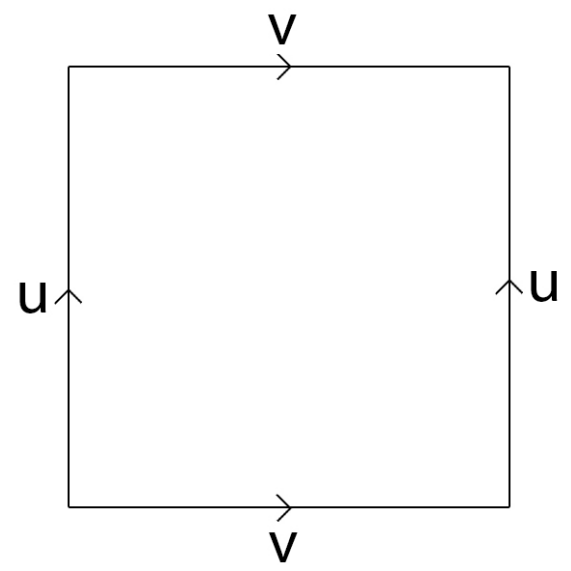

Figura B.4: $\mathbb{K}^{2}$.

Com base em [3, Teorema A.3], nós podemos enunciar o seguinte resultado: 
Proposição B.0.11. $B_{2}\left(\mathbb{K}^{2}\right)$ admite a seguinte apresentação:

Geradores: $a_{1}, a_{2}, \sigma$.

Relações:

(R2) $\sigma^{-1} a_{r} \sigma^{-1} a_{r}=a_{r} \sigma^{-1} a_{r} \sigma, r \in\{1,2\}$

(R3) $\sigma^{-1} a_{1} \sigma a_{2}=a_{2} \sigma^{-1} a_{1} \sigma$,

$(T R) a_{1}^{2} a_{2}^{2}=\sigma^{2}$.

A representação geométrica dos geradores é dada pelas figuras B.5, B.6 e B.\%.

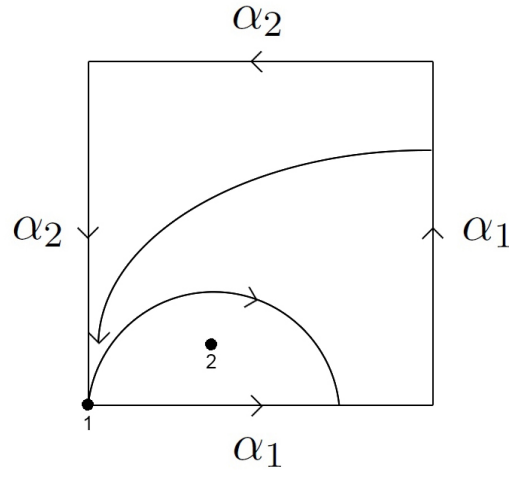

Figura B.5: gerador $a_{1}$.

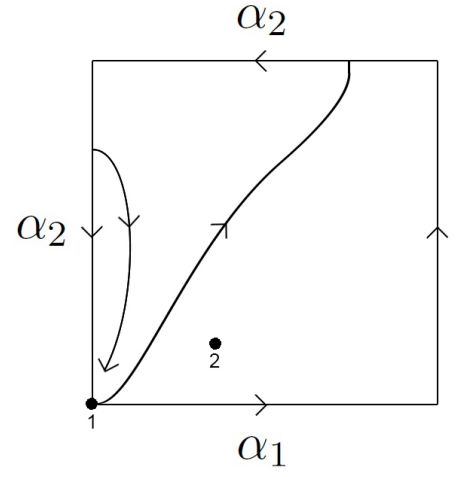

Figura B.6: gerador $a_{2}$.

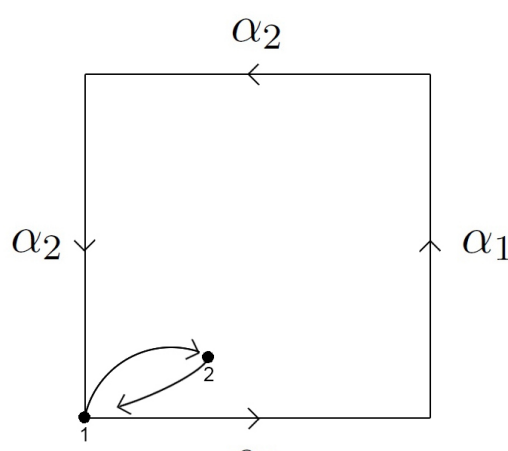

$\alpha_{1}$

Figura B.7: gerador $\sigma$.

Para fornecer uma presentação de $P_{2}\left(\mathbb{K}^{2}\right)$, nós vamos usar o método de Reidemeister-Schreier. Vamos fazer um resumo de como funciona este método, baseado em [24, Apêndice I, Seção 6]. Mais detalhes podem ser encontrados em [21].

Vamos nos restringir ao caso onde $G$ é finitamente presentado e $H$ é de indice finito para ilustrar o método, que vale em geral. Suponhamos que $G=\langle X \mid R\rangle$, sendo $X=\left\{x_{1}, \ldots, x_{n}\right\}$ e $R=\left\{r_{1}, \ldots, r_{m}\right\}$.

Seja $S=\left\{s_{1}=1, s_{2}, \ldots, s_{k}\right\} \subset G$ um conjunto de representantes de classes laterais a direita de $H$, isto é, para cada $g \in G$, existe um único $s_{i} \in S$ tal que $g \in H s_{i}$.

Dizemos que $S$ é um sistema de Schreier de $H$ se vale a seguinte condição:

$$
\begin{gathered}
\text { Se } s_{i}=x_{i_{1}}^{\epsilon_{1}} \ldots x_{i_{-1}}^{\epsilon_{l-1}} x_{i_{l}}^{\epsilon_{l}} \in S \text { está escrito na forma reduzida, isto é, } \\
\epsilon_{i} \in\{1,-1\} \text { e } x_{i_{j}}^{\epsilon_{j}} x_{i_{j+1}}^{\epsilon_{j+1}} \neq 1 \text {, então } x_{i_{1}}^{\epsilon_{1}} \ldots x_{i_{l-1}}^{\epsilon_{l-1}} \in S .
\end{gathered}
$$

Para cada $g \in G$, denotamos por $\bar{g} \in S$ o representante da classe lateral de $g$, ou seja, $g \in H \bar{g}$.

Para cada $s_{i} \in S$ definimos

$$
\varrho\left(s_{i}, g\right)=s_{i} g \overline{s i g}^{-1}
$$

Notemos que como $s_{i} g$ e $\overline{s_{i} g}$ pertencem a mesma classe lateral, então $\varrho\left(s_{i}, g\right) \in H$.

Seja $Y=\left\{\varrho\left(s_{i}, x_{j}\right) \mid s_{i} \in S, x_{j} \in X\right\}$. Se $h \in H$, então temos que

$$
\begin{aligned}
& h=x_{i_{1}}^{\epsilon_{1}} x_{i_{2}}^{\epsilon_{2}} \ldots x_{i_{p-1}}^{\epsilon_{p-1}} x_{i_{p}}^{\epsilon_{p}} \\
& =\left(x_{i_{1}}^{\epsilon_{1}} \overline{x_{i_{1}}^{\epsilon_{1}}}-1\right)\left(\overline{x_{i_{1}}^{\epsilon_{1}}} x_{i_{2}}^{\epsilon_{2}} \overline{x_{i_{1}}^{\epsilon_{1}} x_{i_{2}}^{\epsilon_{2}}}-1\right) \cdots\left(\overline{x_{i_{1}}^{\epsilon_{1}} x_{i_{2}}^{\epsilon_{2}} \cdots x_{i_{p-1}}^{\epsilon_{p-1}}} x_{i_{p}}^{\epsilon_{p}}\right) \\
& =\varrho\left(1, x_{i_{1}}^{\epsilon_{1}}\right) \varrho\left(\overline{x_{i_{1}}^{\epsilon_{1}}}, x_{i_{2}}^{\epsilon_{2}}\right) \varrho\left(\overline{x_{i_{1}}^{\epsilon_{1}} x_{i_{2}}^{\epsilon_{2}}}, x_{i_{3}}^{\epsilon_{3}}\right) \varrho\left(\overline{x_{i_{1}}^{\epsilon_{1}} x_{i_{2}}^{\epsilon_{2}} \ldots x_{i_{p-1}}^{\epsilon_{p-1}}}, x_{i_{p}}^{\epsilon_{p}}\right)=: \tau(h)
\end{aligned}
$$

Seja $W=\left\{\tau\left(s_{i} r_{k} s_{i}^{-1}\right) \mid s_{i} \in S, r_{k} \in R\right\}$. Então $W$ é um conjunto de relações de $Y$.

Em resumo, vale o seguinte resultado: 
Proposição B.0.12 (Método de Reidemeister - Schreier). Sejam $G=\left\langle x_{1}, x_{2}, \ldots, x_{n} \mid r_{1}, \ldots, r_{m}\right\rangle$ um grupo e $H$ um subgrupo de indice finito. Se $S=\left\{s_{1}, \ldots, s_{k}\right\}$ é um sistema de Schreier de $H$, então

$$
H=\left\langle\varrho\left(s_{i}, x_{j}\right) \mid \tau\left(s_{i} r_{k} s_{i}^{-1}\right), i=1, \ldots, k, j=1, \ldots, n, k=1, \ldots, m\right\rangle
$$

sendo $\varrho$ como em (B.1) e $\tau$ como em (B.2).

Agora vamos usar o método acima para calcular uma presentação de $P_{2}\left(\mathbb{K}^{2}\right)$ olhado como um subgrupo de índice dois de $B_{2}\left(\mathbb{K}^{2}\right)$. Um sistema de Schreier para $P_{2}\left(\mathbb{K}^{2}\right)$ é $\{1, \sigma\}$. Vamos calcular os geradores. Temos que

$$
\begin{array}{ll}
\varrho\left(1, a_{1}\right)=1 a_{1}{\overline{1 a_{1}}}^{-1}=a_{1} 1=a_{1}, & \varrho\left(\sigma, a_{1}\right)=\sigma a_{1} \overline{\sigma a_{1}}-1=\sigma a_{1} \sigma^{-1}=: b_{1}, \\
\varrho\left(1, a_{2}\right)=1 a_{2}{\overline{1 a_{2}}}^{-1}=a_{2} 1=a_{2}, & \varrho\left(\sigma, a_{2}\right)=\sigma a_{2} \overline{\sigma a}^{-1}=\sigma a_{2} \sigma^{-1}=: b_{2}, \\
\varrho(1, \sigma)=1 \sigma \overline{1 \sigma}^{-1}=\sigma \sigma^{-1}=1, & \varrho(\sigma, \sigma)=\sigma \sigma \overline{\sigma \sigma}-1=\sigma^{2} 1=\sigma^{2}=: B .
\end{array}
$$

Agora vamos calcular as relações. Temos que

$$
\begin{aligned}
& \tau\left(1(R 2) 1^{-1}\right)=\tau\left(\sigma^{-1} a_{r} \sigma^{-1} a_{r} \sigma^{-1} a_{r}^{-1} \sigma a_{r}^{-1}\right) \\
& =\left(\sigma^{-1}{\overline{\sigma^{-1}}}^{-1}\right)\left(\overline{\sigma^{-1}} a_{r}{\overline{\sigma^{-1} a_{r}}}^{-1}\right) \\
& \left(\overline{\sigma^{-1} a_{r}} \sigma^{-1}{\overline{\sigma^{-1} a_{r} \sigma^{-1}}}^{-1}\right) \\
& \left(\overline{\sigma^{-1} a_{r} \sigma^{-1}} a_{r} \overline{\sigma^{-1} a_{r} \sigma^{-1} a_{r}}-1\right) \\
& \left(\overline{\sigma^{-1} a_{r} \sigma^{-1} a_{r}} \sigma^{-1} \overline{\sigma^{-1} a_{r} \sigma^{-1} a_{r} \sigma^{-1}}{ }^{-1}\right) \\
& \left(\overline{\sigma^{-1} a_{r} \sigma^{-1} a_{r} \sigma^{-1}} a_{r}^{-1} \overline{\sigma^{-1} a_{r} \sigma^{-1} a_{r} \sigma^{-1} a_{r}^{-1}}{ }^{-1}\right) \\
& \left(\overline{\sigma^{-1} a_{r} \sigma^{-1} a_{r} \sigma^{-1} a_{r}^{-1}} \sigma \overline{\sigma^{-1} a_{r} \sigma^{-1} a_{r} \sigma^{-1} a_{r}^{-1} \sigma^{-1}}\right) \\
& \left(\overline{\sigma^{-1} a_{r} \sigma^{-1} a_{r} \sigma^{-1} a_{r}^{-1} \sigma} a_{r}^{-1} \overline{\sigma^{-1} a_{r} \sigma^{-1} a_{r} \sigma^{-1} a_{r}^{-1} \sigma a_{r}^{-1}}{ }^{-1}\right) \\
& =\left(\sigma^{-1} \sigma^{-1}\right)\left(\sigma a_{r} \sigma^{-1}\right)\left(\sigma \sigma^{-1} 1\right)\left(1 a_{r} 1\right)\left(1 \sigma^{-1} \sigma^{-1}\right)\left(\sigma a_{r}^{-1} \sigma^{-1}\right)(\sigma \sigma 1)\left(1 a_{r}^{-1} 1\right) \\
& =B^{-1} b_{r} a_{r} B^{-1} b_{r}^{-1} B a_{r}^{-1} \text {, } \\
& \tau\left(\sigma(R 2) \sigma^{-1}\right)=\tau\left(a_{r} \sigma^{-1} a_{r} \sigma^{-1} a_{r}^{-1} \sigma a_{r}^{-1} \sigma^{-1}\right) \\
& =\left(a_{r}{\overline{a_{r}}}^{-1}\right)\left(\overline{a_{r}} \sigma^{-1}{\overline{a_{r} \sigma^{-1}}}^{-1}\right) \\
& \left(\overline{a_{r} \sigma^{-1}} a_{r}{\overline{a_{r} \sigma^{-1} a_{r}}}^{-1}\right) \\
& \left(\overline{a_{r} \sigma^{-1} a_{r}} \sigma^{-1} \overline{a_{r} \sigma^{-1} a_{r} \sigma^{-1}}-1\right) \\
& \left(\overline{a_{r} \sigma^{-1} a_{r} \sigma^{-1}} a_{r}^{-1} \overline{a_{r} \sigma^{-1} a_{r} \sigma^{-1} a_{r}^{-1}}{ }^{-1}\right) \\
& \left(\overline{a_{r} \sigma^{-1} a_{r} \sigma^{-1} a_{r}^{-1}} \sigma{\overline{a_{r} \sigma^{-1} a_{r} \sigma^{-1} a_{r}^{-1} \sigma}}^{-1}\right) \\
& \left(\overline{a_{r} \sigma^{-1} a_{r} \sigma^{-1} a_{r}^{-1} \sigma} a_{r}^{-1} \overline{a_{r} \sigma^{-1} a_{r} \sigma^{-1} a_{r}^{-1} \sigma a_{r}^{-1}}{ }^{-1}\right) \\
& \left(\overline{a_{r} \sigma^{-1} a_{r} \sigma^{-1} a_{r}^{-1} \sigma a_{r}^{-1}} \sigma^{-1} \overline{a_{r} \sigma^{-1} a_{r} \sigma^{-1} a_{r}^{-1} \sigma a_{r}^{-1} \sigma^{-1}}{ }^{-1}\right) \\
& =\left(a_{r} 1\right)\left(1 \sigma^{-1} \sigma^{-1}\right)\left(\sigma a_{r} \sigma^{-1}\right)\left(\sigma \sigma^{-1} 1\right)\left(1 a_{r}^{-1} 1\right)\left(1 \sigma \sigma^{-1}\right)\left(\sigma a_{r}^{-1} \sigma^{-1}\right)\left(\sigma \sigma^{-1} 1\right) \\
& =a_{r} B^{-1} b_{r} a_{r}^{-1} b_{r}^{-1} \text {, }
\end{aligned}
$$




$$
\begin{aligned}
& \tau\left(1(R 3) 1^{-1}\right)=\tau\left(\sigma^{-1} a_{1} \sigma a_{2} \sigma^{-1} a_{1}^{-1} \sigma a_{2}^{-1}\right) \\
& =\left(\sigma^{-1} \overline{\sigma^{-1}}\right)\left(\overline{\sigma^{-1}} a_{1}{\overline{\sigma^{-1} a_{1}}}^{-1}\right) \\
& \left(\overline{\sigma^{-1} a_{1}} \sigma{\overline{\sigma^{-1} a_{1} \sigma}}^{-1}\right) \\
& \left(\overline{\sigma^{-1} a_{1} \sigma} a_{2}{\overline{\sigma^{-1} a_{1} \sigma a_{2}}}^{-1}\right) \\
& \left(\overline{\sigma^{-1} a_{1} \sigma a_{2}} \sigma^{-1}{\overline{\sigma^{-1} a_{1} \sigma a_{2} \sigma^{-1}}}^{-1}\right) \\
& \left(\overline{\sigma^{-1} a_{1} \sigma a_{2} \sigma^{-1}} a_{1}^{-1} \overline{\sigma^{-1} a_{1} \sigma a_{2} \sigma^{-1} a_{1}^{-1}}\right) \\
& \left(\overline{\sigma^{-1} a_{1} \sigma a_{2} \sigma^{-1} a_{1}^{-1}} \sigma{\overline{\sigma^{-1} a_{1} \sigma a_{2} \sigma^{-1} a_{1}^{-1} \sigma}}^{-1}\right) \\
& \left.\overline{\left(\sigma^{-1} a_{1} \sigma a_{2} \sigma^{-1} a_{1}^{-1} \sigma\right.} a_{2}^{-1} \overline{\sigma^{-1} a_{1} \sigma a_{2} \sigma^{-1} a_{1}^{-1} \sigma a_{2}^{-1}}{ }^{-1}\right) \\
& =\left(\sigma^{-1} \sigma^{-1}\right)\left(\sigma a_{1} \sigma^{-1}\right)\left(\sigma \sigma^{-1}\right)\left(1 a_{2} 1\right)\left(1 \sigma^{-1} \sigma^{-1}\right)\left(\sigma a_{1}^{-1} \sigma^{-1}\right)(\sigma \sigma 1)\left(1 a_{2}^{-1} 1\right) \\
& =B^{-1} b_{1} B a_{2} B^{-1} b_{1}^{-1} B a_{2}^{-1} \text {, } \\
& \tau\left(\sigma(R 3) \sigma^{-1}\right)=\tau\left(a_{1} \sigma a_{2} \sigma^{-1} a_{1}^{-1} \sigma a_{2}^{-1} \sigma^{-1}\right) \\
& =\left(a_{1}{\overline{a_{1}}}^{-1}\right)\left(\overline{a_{1}} \sigma{\overline{a_{1}}}^{-1}\right) \\
& \left(\overline{a_{1} \sigma} a_{2}{\overline{a_{1} \sigma a_{2}}}^{-1}\right) \\
& \left(\overline{a_{1} \sigma a_{2}} \sigma^{-1}{\overline{a_{1} \sigma a_{2} \sigma^{-1}}}^{-1}\right) \\
& \left(\overline{a_{1} \sigma a_{2} \sigma^{-1}} a_{1}^{-1} \overline{a_{1} \sigma a_{2} \sigma^{-1} a_{1}^{-1}}{ }^{-1}\right) \\
& \left(\overline{a_{1} \sigma a_{2} \sigma^{-1} a_{1}^{-1}} \sigma \overline{a_{1} \sigma a_{2} \sigma^{-1} a_{1}^{-1} \sigma}{ }^{-1}\right) \\
& \left.\overline{\left(a_{1} \sigma a_{2} \sigma^{-1} a_{1}^{-1} \sigma\right.} a_{2}^{-1}{\overline{a_{1} \sigma a_{2} \sigma^{-1} a_{1}^{-1} \sigma a_{2}^{-1}}}^{-1}\right) \\
& \left.\overline{\left(a_{1} \sigma a_{2} \sigma^{-1} a_{1}^{-1} \sigma a_{2}^{-1}\right.} \sigma^{-1} \overline{a_{1} \sigma a_{2} \sigma^{-1} a_{1}^{-1} \sigma a_{2}^{-1} \sigma^{-1}}{ }^{-1}\right) \\
& =\left(a_{1} 1\right)\left(1 \sigma \sigma^{-1}\right)\left(\sigma a_{2} \sigma^{-1}\right)\left(\sigma \sigma^{-1} 1\right)\left(1 a_{1}^{-1} 1\right)\left(1 \sigma \sigma^{-1}\right)\left(\sigma a_{2}^{-1} \sigma^{-1}\right)\left(\sigma \sigma^{-1} 1\right) \\
& =a_{1} b_{2} a_{1}^{-1} b_{2}^{-1} \text {, } \\
& \begin{aligned}
\tau\left(1(T R) 1^{-1}\right)= & \tau\left(a_{1} a_{1} a_{2} a_{2} \sigma^{-1} \sigma^{-1}\right) \\
= & =\left(a_{1} \overline{a_{1}}-1\right)\left(\overline{a_{1}} a_{1} \overline{a_{1} a_{1}}-1\right) \\
& \left(\overline{a_{1} a_{1}} a_{2} \overline{a_{1} a_{1} a_{2}}-1\right) \\
& \left(\overline{a_{1} a_{1} a_{2}} a_{2} \overline{a_{1} a_{1} a_{2} a_{2}}-1\right) \\
& \left(\overline{a_{1} a_{1} a_{2} a_{2}} \sigma^{-1} \overline{a_{1} a_{1} a_{2} a_{2} \sigma^{-1}}-1\right) \\
& \left(\overline{a_{1} a_{1} a_{2} a_{2} \sigma^{-1}} \sigma^{-1} \overline{a_{1} a_{1} a_{2} a_{2} \sigma^{-1} \sigma^{-1}}-1\right) \\
= & \left(a_{1} 1\right)\left(1 a_{1} 1\right)\left(1 a_{2} 1\right)\left(1 a_{2} 1\right)\left(1 \sigma^{-1} \sigma^{-1}\right)\left(\sigma \sigma^{-1} 1\right) \\
= & a_{1} a_{1} a_{2} a_{2} B^{-1},
\end{aligned}
\end{aligned}
$$




$$
\begin{aligned}
\tau\left(\sigma(T R) \sigma^{-1}\right)= & \left(\sigma a_{1} a_{1} a_{2} a_{2} \sigma^{-1} \sigma^{-1} \sigma^{-1}\right) \\
= & (\sigma \bar{\sigma}-1)\left(\bar{\sigma} a_{1} \overline{\sigma a}_{1}^{-1}\right) \\
& \left(\overline{\sigma a_{1}} a_{1} \overline{\sigma a_{1} a_{1}}-1\right) \\
& \left(\overline{\sigma a_{1} a_{1}} a_{2}{\overline{a_{1} a_{1} a_{2}}}^{-1}\right) \\
& \left(\overline{\sigma a_{1} a_{1} a_{2}} a_{2} \overline{\sigma a_{1} a_{1} a_{2} a_{2}}-1\right) \\
& \left(\overline{\sigma a_{1} a_{1} a_{2} a_{2}} \sigma^{-1} \overline{\sigma a_{1} a_{1} a_{2} a_{2} \sigma^{-1}}\right) \\
& \left(\overline{\sigma a_{1} a_{1} a_{2} a_{2} \sigma^{-1}} \sigma^{-1} \overline{\sigma a_{1} a_{1} a_{2} a_{2} \sigma^{-1} \sigma^{-1}}-1\right) \\
& \left(\overline{\sigma a_{1} a_{1} a_{2} a_{2} \sigma^{-1} \sigma^{-1}} \sigma^{-1} \overline{\sigma a_{1} a_{1} a_{2} a_{2} \sigma^{-1} \sigma^{-1} \sigma^{-1}}{ }^{-1}\right) \\
= & \left(\sigma \sigma^{-1}\right)\left(\sigma a_{1} \sigma^{-1}\right)\left(\sigma a_{1} \sigma^{-1}\right)\left(\sigma a_{2} \sigma^{-1}\right)\left(\sigma a_{2} \sigma^{-1}\right)\left(\sigma \sigma^{-1} 1\right)\left(1 \sigma^{-1} \sigma^{-1}\right)\left(\sigma \sigma^{-1} 1\right) \\
= & b_{1} b_{1} b_{2} b_{2} B^{-1} .
\end{aligned}
$$

Rearrumando a maneira de escrever as relações, nós obtemos o seguinte resultado:

Teorema B.0.13. $P_{2}\left(\mathbb{K}^{2}\right)$ admite a seguinte apresentação:

Geradores: $a_{1}, a_{2}, b_{1}, b_{2}, B$.

Relações:

(i) $b_{r} a_{r}=B a_{r} B^{-1} b_{r} B, r \in\{1,2\}$;

(ii) $\left[a_{r}, b_{r}\right]=a_{r} B a_{r}^{-1}, r \in\{1,2\}$;

(iii) $b_{1} B a_{2} B^{-1}=B a_{2} B^{-1} b_{1}$;

(iv) $\left[a_{1}, b_{2}\right]=1$;

(v) $a_{1}^{2} a_{2}^{2}=b_{1}^{2} b_{2}^{2}=B$.

Lembremos que $p_{1}: F_{2}\left(\mathbb{K}^{2}\right) \rightarrow \mathbb{K}^{2}$ é a projeção no primeiro fator, isto é, para cada $(x, y) \in F_{2}\left(\mathbb{K}^{2}\right)$, temos que $p_{1}(x, y)=x$. A partir dos modelos geométricos dos geradores $a_{1}, a_{2}, \sigma \in B_{2}\left(\mathbb{K}^{2}\right)$, segue que o homomorfismo

$$
\left(p_{1}\right)_{\#}: P_{2}\left(\mathbb{K}^{2}\right) \longrightarrow \pi_{1}\left(\mathbb{K}^{2}\right)=\left\langle\alpha_{1}, \alpha_{2} \mid \alpha_{1}^{2} \alpha_{2}^{2}=1\right\rangle
$$

é definido nos geradores do seguinte modo:

$$
\begin{array}{llrl}
\left(p_{1}\right)_{\#}\left(a_{1}\right)=\alpha_{1}^{-1} & \left(p_{1}\right)_{\#}\left(b_{1}\right)=1 & \left(p_{1}\right)_{\#}(B)=1 . \\
\left(p_{1}\right)_{\#}\left(a_{2}\right)=\alpha_{2}^{-1} & \left(p_{1}\right)_{\#}\left(b_{2}\right)=1 &
\end{array}
$$

Vamos dar uma seção para o homomorfismo $\left(p_{1}\right)_{\#}$, isto é, um homomorfismo

$$
\beta: \pi_{1}\left(\mathbb{K}^{2}\right) \rightarrow P_{2}\left(\mathbb{K}^{2}\right) \quad \text { tal que }\left(p_{1}\right)_{\#} \circ \beta=I d .
$$

Definimos

$$
\beta:\left\{\begin{array}{l}
\alpha_{1} \longmapsto a_{1}^{-1} b_{1}^{-1} \\
\alpha_{2} \longmapsto a_{2}^{-1} b_{2}^{-1} .
\end{array}\right.
$$


De fato $\beta$ define um homomorfismo, pois usando as relações do Teorema B.0.13, temos que

$$
\begin{aligned}
& \left.\beta\left(\alpha_{1}^{2} \alpha_{2}^{2}\right)=\left(a_{1}^{-1} b_{1}^{-1}\right)^{2}\left(a_{2}^{-1} b_{2}^{-1}\right)^{2}=\left(\left(b_{2} a_{2}\right)^{2}\left(b_{1} a_{1}\right)^{2}\right)\right)^{-1} \\
& =\left(b_{2} a_{2}\left(b_{2} a_{2}\right)\left(b_{1} a_{1}\right) b_{1} a_{1}\right)^{-1}=\left(b_{2} a_{2}\left[b_{2}, a_{2}\right] a_{2} b_{2}\left[b_{1}, a_{1}\right] a_{1} b_{1} b_{1} a_{1}\right)^{-1} \\
& \stackrel{(i i)}{=}\left(b_{2} a_{2} a_{2} B^{-1} a_{2}^{-1} a_{2} b_{2} a_{1} B^{-1} a_{1}^{-1} a_{1} b_{1} b_{1} a_{1}\right)^{-1}=\left(b_{2}\left(a_{2}^{2}\right) B^{-1} b_{2} a_{1} B^{-1}\left(b_{1}^{2}\right) a_{1}\right)^{-1} \\
& \stackrel{(v)}{=}\left(b_{2} a_{1}^{-2} B B^{-1} b_{2} a_{1} B^{-1} B b_{2}^{-2} a_{1}\right)^{-1}=\left(b_{2} a_{1}^{-2} b_{2} a_{1} b_{2}^{-2} a_{1}\right)^{-1} \\
& \stackrel{(i v)}{=} 1 \text {. }
\end{aligned}
$$

Mostremos que $\beta$ é de fato uma seção. Temos que

$$
\left(\left(p_{1}\right)_{\#} \circ \beta\right)\left(\alpha_{1}\right)=\left(p_{1}\right)_{\#}\left(a_{1}^{-1} b_{1}^{-1}\right)=\left(p_{1}\right)_{\#}\left(a_{1}\right)^{-1}\left(p_{1}\right)_{\#}\left(b_{1}\right)^{-1}=\left(\alpha_{1}^{-1}\right)^{-1} 1=\alpha_{1}
$$

e

$$
\left(\left(p_{1}\right)_{\#} \circ \beta\right)\left(\alpha_{2}\right)=\left(p_{1}\right)_{\#}\left(a_{2}^{-1} b_{2}^{-1}\right)=\left(p_{1}\right)_{\#}\left(a_{2}\right)^{-1}\left(p_{1}\right)_{\#}\left(b_{2}\right)^{-1}=\left(\alpha_{2}^{-1}\right)^{-1} 1=\alpha_{2} .
$$

Lembremos que as funções

$$
\left\langle\alpha_{1}, \alpha_{2} \mid \alpha_{1}^{2} \alpha_{2}^{2}=1\right\rangle \stackrel{r}{\underset{s}{\rightleftarrows}} \mathbb{Z} \rtimes \mathbb{Z}
$$

definidas, respectivamente por

$$
r:\left\{\begin{array}{l}
\alpha_{1} \longmapsto(1,-1) \\
\alpha_{2} \longmapsto(0,1)
\end{array} \quad s:\left\{\begin{array}{l}
(1,0) \longmapsto \alpha_{1}^{-1} \alpha_{2}^{-1} \\
(0,1) \longmapsto \alpha_{2}
\end{array}\right.\right.
$$

são isomorfismo e $r=s^{-1}$. Logo, a seguinte sequência é exata e cinde:

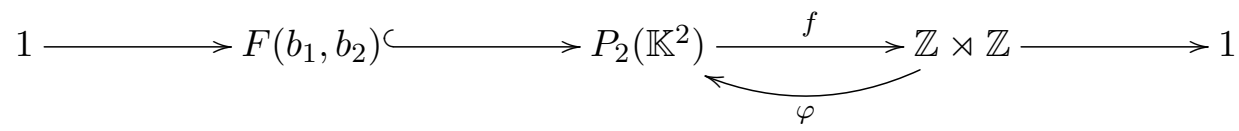

sendo $f=r \circ\left(p_{1}\right)_{\#}$ e $\varphi=\beta \circ s$.

Assim, nós temos um bem definido homomorfismo

$$
\begin{aligned}
\theta: \mathbb{Z} \rtimes \mathbb{Z} & \longrightarrow \operatorname{Aut}\left(F\left(b_{1}, b_{2}\right)\right) \\
\theta(m, n): \quad F\left(b_{1}, b_{2}\right) & \longrightarrow F\left(b_{1}, b_{2}\right) \\
x & \longmapsto \varphi(m, n) x \varphi(m, n)^{-1} .
\end{aligned}
$$

Lembremos que

$$
\begin{aligned}
& F\left(b_{1}, b_{2}\right) \rtimes_{\theta}(\mathbb{Z} \rtimes \mathbb{Z}) \underset{\gamma}{\stackrel{\lambda}{\rightleftarrows}} P_{2}\left(\mathbb{K}^{2}\right) \\
& \lambda(b, c)=b \varphi(c) \\
& \gamma(x)=\left(x \varphi\left(f\left(x^{-1}\right)\right), f(x)\right)
\end{aligned}
$$

são isomorfismos e $\lambda=\gamma^{-1}$.

Afim de calcular explicitamente quanto vale $\theta(m, n)$ em um elemento de $F\left(b_{1}, b_{2}\right)$ para cada $(m, n) \in \mathbb{Z} \rtimes \mathbb{Z}$, nós precisamos saber como os elementos $a_{1}, a_{2}$ agem em $b_{1}, b_{2}$. Porém também calcularemos explicitamente como $a_{1}, a_{2}$ agem em $B$, pois usaremos nos cálculos posteriores.

Lema B.0.14. Em $P_{2}\left(\mathbb{K}^{2}\right)$ valem as seguintes relações: 
(1) $a_{1} b_{1} a_{1}^{-1}=b_{1}^{-1} b_{2}^{-2}=b_{1}^{-1} B^{-1} b_{1}^{2}$;

(2) $a_{1} b_{2} a_{1}^{-1}=b_{2}$;

(3) $a_{1} B a_{1}^{-1}=b_{1}^{-1} b_{2}^{-2} b_{1}^{-1}=b_{1}^{-1} B b_{1}$;

(4) $a_{2} b_{1} a_{2}^{-1}=\left(b_{2}^{-1} B^{-1} b_{2}^{-1}\right) b_{1}\left(b_{2} B b_{2}\right)$;

(5) $a_{2} b_{2} a_{2}^{-1}=b_{2}^{-1} B^{-1} b_{2}^{2}$;

(6) $a_{2} B a_{2}^{-1}=b_{2}^{-1} B^{-1} b_{2}$;

(7) $a_{1}^{-1} b_{1} a_{1}=B^{-1} b_{1}$;

(8) $a_{1}^{-1} b_{2} a_{1}=b_{2}$;

(9) $a_{1}^{-1} B a_{1}=\left(b_{2}^{-2} b_{1}^{-1}\right)^{2} b_{2}^{2}=\left(B^{-1} b_{1}\right) B^{-1}\left(b_{1}^{-1} B\right)$;

(10) $a_{2}^{-1} b_{1} a_{2}=\left(B^{-1} b_{2}^{-1} b_{1}^{-2} b_{2}^{-1}\right) b_{1}\left(b_{2} b_{1}^{2} b_{2} B\right)=\left(B^{-1} b_{2} B^{-1} b_{2}\right) b_{1}^{2}\left(b_{2}^{-1} B b_{2}^{-1} B\right)$;

(11) $a_{2}^{-1} b_{2} a_{2}=B^{-1} b_{2}$;

(12) $a_{2}^{-1} B a_{2}=B^{-1} b_{2}^{-1} b_{1}^{-2} b_{2}^{-1} B=\left(B^{-1} b_{2}\right) B^{-1}\left(b_{2}^{-1} B\right)$.

Demonstração. Vamos usar as relações dadas no Teorema B.0.13 para mostrar que valem as fórmulas como no enunciado do Lema. As relações (2) e (8) seguem diretamente da relação (iv). Temos que

$$
\begin{aligned}
{\left[a_{r}, b_{r}\right] } & \stackrel{\stackrel{(i i)}{=}}{=} a_{r} B a_{r}^{-1} \\
a_{r} b_{r} a_{r}^{-1} b_{r}^{-1} & =a_{r} B a_{r}^{-1} \\
b_{r} a_{r}^{-1} b_{r}^{-1} & =B a_{r}^{-1} \\
a_{r}^{-1} b_{r}^{-1} a_{r} & =b_{r}^{-1} B \\
a_{r}^{-1} b_{r} a_{r} & =B^{-1} b_{r}
\end{aligned}
$$

e portanto, valem as igualdades (7) e (11). Agora temos que

$$
a_{1}^{-1} B a_{1} \stackrel{(v)}{=} a_{1}^{-1} b_{1}^{2} b_{2}^{2} a_{1} \stackrel{(i v)}{=} a_{1}^{-1} b_{1}^{2} a_{1} b_{2}^{2}=\left(a_{1}^{-1} b_{1} a_{1}\right)^{2} b_{2}^{2} \stackrel{(7)}{=}\left(B^{-1} b_{1}\right)^{2} b_{2}^{2} \stackrel{(v)}{=}\left(b_{2}^{-2} b_{1}^{-2} b_{1}\right)^{2} b_{2}^{2}=\left(b_{2}^{-2} b_{1}^{-1}\right)^{2} b_{2}^{2}
$$

e logo, vale (9). Mostremos que vale (1).

$$
\begin{aligned}
{\left[a_{1}, b_{1}\right] } & \stackrel{(i i)}{=} a_{1} B a_{1}^{-1} \\
a_{1} b_{1} a_{1}^{-1} b_{1}^{-1} & =a_{1} B a_{1}^{-1} \\
a_{1} b_{1} a_{1}^{-1} & =a_{1} B a_{1}^{-1} b_{1} \\
a_{1} b_{1} a_{1}^{-1} & \stackrel{(v)}{=} a_{1} b_{1}^{2} b_{2}^{2} a_{1}^{-1} b_{1} \\
a_{1} b_{1} a_{1}^{-1} & \stackrel{(i v)}{=} a_{1} b_{1}^{2} a_{1}^{-1} b_{2}^{2} b_{1} \\
a_{1} b_{1} a_{1}^{-1} & =\left(a_{1} b_{1} a_{1}^{-1}\right)^{2} b_{2}^{2} b_{1} \\
b_{1}^{-1} b_{2}^{-1} & =a_{1} b_{1} a_{1}^{-1} .
\end{aligned}
$$

Mostremos agora que vale (3).

$a_{1} B a_{1}^{-1} \stackrel{(v)}{=} a_{1} b_{1}^{2} b_{2}^{2} a_{1}^{-1} \stackrel{(i v)}{=} a_{1} b_{1}^{2} a_{1}^{-1} b_{2}^{2}=\left(a_{1} b_{1} a_{1}^{-1}\right)^{2} b_{2}^{2} \stackrel{(1)}{=}\left(b_{1}^{-1} b_{2}^{-2}\right)^{2} b_{2}^{2}=b_{1}^{-1} b_{2}^{-2} b_{1}^{-1} b_{2}^{-2} b_{2}^{2}=b_{1}^{-1} b_{2}^{-2} b_{1}^{-1}$. 
Temos também que

$$
\begin{aligned}
b_{2} a_{2} & \stackrel{(i)}{=} B a_{2} B^{-1} b_{2} B \\
B^{-1} b_{2} a_{2} B^{-1} b_{2}^{-1} B a_{2}^{-1} & =1 \\
B^{-1} b_{2}\left(a_{2} B a_{2}^{-1}\right)^{-1}\left(a_{2} b_{2} a_{2}^{-1}\right)^{-1}\left(a_{2} B a_{2}^{-1}\right) & =1 \\
B^{-1} b_{2}\left[a_{2}, b_{2}\right]^{-1}\left(a_{2} b_{2} a_{2}^{-1}\right)^{-1}\left[a_{2}, b_{2}\right] & \stackrel{(i i)}{=} 1 \\
B^{-1} b_{2} b_{2}\left(a_{2} b_{2}^{-1} a_{2}^{-1}\right)\left(a_{2} b_{2} a_{2}^{-1}\right)^{-1}\left(a_{2} b_{2} a_{2}^{-1}\right) b_{2}^{-1} & =1 \\
B^{-1} b_{2}^{2}\left(a_{2} b_{2}^{-1} a_{2}^{-1}\right) b_{2}^{-1} & =1 \\
a_{2} b_{2}^{-1} a_{2}^{-1} & =b_{2}^{-2} B b_{2} \\
a_{2} b_{2} a_{2}^{-1} & =b_{2}^{-1} B^{-1} b_{2}^{2} \\
&
\end{aligned}
$$

e portanto, vale (5). Mostremos que vale (6).

$$
\begin{aligned}
{\left[a_{2}, b_{2}\right] } & \stackrel{(i i)}{=} a_{2} B a_{2}^{-1} \\
a_{2} b_{2} a_{2}^{-1} b_{2}^{-1} & =a_{2} B a_{2}^{-1} \\
b_{2}^{-1} B^{-1} b_{2}^{2} b_{2}^{-1} & \stackrel{(5)}{=} a_{2} B a_{2}^{-1} \\
b_{2}^{-1} B^{-1} b_{2} & =a_{2} B a_{2}^{-1} .
\end{aligned}
$$

Vamos mostrar que vale (4).

$$
\begin{aligned}
b_{1} B a_{2} B^{-1} & \stackrel{(i i i)}{=} B a_{2} B^{-1} b_{1} \\
b_{1} B a_{2} B^{-1} b_{1}^{-1} B a_{2}^{-1} & =B \\
b_{1} B\left(a_{2} B a_{2}^{-1}\right)^{-1}\left(a_{2} b_{1} a_{2}^{-1}\right)^{-1}\left(a_{2} B a_{2}^{-1}\right) & =B \\
b_{1} B b_{2}^{-1} B b_{2}\left(a_{2} b_{1} a_{2}^{-1}\right)^{-1} b_{2}^{-1} B^{-1} b_{2} & \stackrel{(6)}{=} B \\
\left(a_{2} b_{1} a_{2}^{-1}\right)^{-1} & =b_{2}^{-1} B^{-1} b_{2} B^{-1} b_{1}^{-1} B b_{2}^{-1} B b_{2} \\
\left(a_{2} b_{1} a_{2}^{-1}\right)^{-1} & \stackrel{(v)}{=} b_{2}^{-1} B^{-1} b_{2} b_{2}^{-2} b_{1}^{-2} b_{1}^{-1} b_{1}^{2} b_{2}^{2} b_{2}^{-1} B b_{2} \\
\left(a_{2} b_{1} a_{2}^{-1}\right)^{-1} & =b_{2}^{-1} B^{-1} b_{2}^{-1} b_{1}^{-1} b_{2} B b_{2} \\
a_{2} b_{1} a_{2}^{-1} & =b_{2}^{-1} B^{-1} b_{2}^{-1} b_{1} b_{2} B b_{2} . \\
&
\end{aligned}
$$

Agora temos que

$$
\begin{aligned}
a_{2} B a_{2}^{-1} & \stackrel{(6)}{=} b_{2}^{-1} B^{-1} b_{2} \\
b_{2} a_{2} B a_{2}^{-1} b_{2}^{-1} & =B^{-1} \\
a_{2}^{-1} b_{2} a_{2} B a_{2}^{-1} b_{2}^{-1} a_{2} & =a_{2}^{-1} B^{-1} a_{2} \\
B^{-1} b_{2} B b_{2}^{-1} B & \stackrel{(11)}{=} a_{2}^{-1} B^{-1} a_{2} \\
a_{2}^{-1} B a_{2} & =B^{-1} b_{2} B^{-1} b_{2}^{-1} B \\
a_{2}^{-1} B a_{2} & \stackrel{(v)}{=} B^{-1} b_{2} b_{2}^{-2} b_{1}^{-2} b_{2}^{-1} B \\
a_{2}^{-1} B a_{2} & =B^{-1} b_{2}^{-1} b_{1}^{-2} b_{2}^{-1} B
\end{aligned}
$$


e portanto, vale (12). Por fim, mostremos que vale (10), o que encerra a demonstração.

$$
\begin{aligned}
& b_{1} B a_{2} B^{-1} \stackrel{(i i i)}{=} B a_{2} B^{-1} b_{1} \\
&\left(a_{2}^{-1} b_{1} a_{2}\right)\left(a_{2}^{-1} B a_{2}\right) B^{-1}=\left(a_{2}^{-1} B a_{2}\right) B^{-1} b_{1} \\
& a_{2}^{-1} b_{1} a_{2}=\left(a_{2}^{-1} B a_{2}\right) B^{-1} b_{1} B\left(a_{2}^{-1} B a_{2}\right)^{-1} \\
& a_{2}^{-1} b_{1} a_{2} \stackrel{(12)}{=} \\
& a_{2}^{-1} b_{1} a_{2} B^{-1} b_{2}^{-1} b_{1}^{-2} b_{2}^{-1} B B^{-1} b_{1} B B^{-1} b_{2} b_{1}^{2} b_{2} B \\
& B^{-1} b_{2}^{-1} b_{1}^{-2} b_{2}^{-1} b_{1} b_{2} b_{1}^{2} b_{2} B .
\end{aligned}
$$

Vamos calcular explicitamente $f$ nos geradores de $P_{2}\left(\mathbb{K}^{2}\right)$ e $\varphi$ nos geradores de $\mathbb{Z} \rtimes \mathbb{Z}$.

$$
\begin{gathered}
f\left(a_{1}\right)=\left(r \circ\left(p_{1}\right)_{\#}\right)\left(a_{1}\right)=r\left(\alpha_{1}^{-1}\right)=r\left(\alpha_{1}\right)^{-1}=(1,-1)^{-1}=(1,1) \\
f\left(a_{2}\right)=\left(r \circ\left(p_{1}\right)_{\#}\right)\left(a_{2}\right)=r\left(\alpha_{2}^{-1}\right)=r(\alpha)_{2}^{-1}=(0,1)^{-1}=(0,-1) \\
f\left(b_{i}\right)=\left(r \circ\left(p_{1}\right)_{\#}\right)\left(b_{i}\right)=r(1)=(0,0), i \in\{1,2\} \\
\varphi(1,0)=(\beta \circ s)(1,0)=\beta\left(\alpha_{1}^{-1} \alpha_{2}^{-1}\right)=\beta\left(\alpha_{1}\right)^{-1} \beta\left(\alpha_{2}\right)^{-1} \\
=\left(a_{1}^{-1} b_{1}^{-1}\right)^{-1}\left(a_{2}^{-1} b_{2}^{-1}\right)^{-1}=b_{1} a_{1} b_{2} a_{2} \stackrel{(i v)}{=} b_{1} b_{2} a_{1} a_{2} \\
\varphi(0,1)=(\beta \circ s)(0,1)=\beta\left(\alpha_{2}\right)=a_{2}^{-1} b_{2}^{-1}=b_{2}^{-1} B a_{2}^{-1} .
\end{gathered}
$$

Agora vamos calcular $\theta(1,0)$ e $\theta(0,1)$ nos geradores de $F\left(b_{1}, b_{2}\right)$. Usaremos o Teorema B.0.13 e o Lema B.0.14

$$
\begin{aligned}
\theta(1,0)\left(b_{1}\right) & =\quad(1,0) b_{1} \varphi(1,0)^{-1}=b_{1} a_{1} b_{2}\left(a_{2} b_{1} a_{2}^{-1}\right) b_{2}^{-1} a_{1}^{-1} b_{1}^{-1} \\
& \stackrel{(4)}{=} \quad b_{1} a_{1} b_{2} b_{2}^{-1} B^{-1} b_{2}^{-1} b_{1} b_{2} B b_{2} b_{2}^{-1} a_{1}^{-1} b_{1}^{-1} \\
& \stackrel{(i v)}{=} \quad b_{1}\left(a_{1} B a_{1}^{-1}\right)^{-1} b_{2}^{-1}\left(a_{1} b_{1} a_{1}^{-1}\right) b_{2}\left(a_{1} B a_{1}^{-1}\right) b_{1}^{-1} \\
& \stackrel{(1,3)}{=} \quad\left(b_{1} b_{1} b_{2}^{2}\right) b_{1} b_{2}^{-1} b_{1}^{-1} b_{2}^{-2} b_{2} b_{1}^{-1}\left(b_{2}^{-2} b_{1}^{-1} b_{1}^{-1}\right) \\
& \stackrel{(v)}{=} \quad B b_{1} b_{2}^{-1} b_{1}^{-1} b_{2}^{-1} b_{1}^{-1} B^{-1} \\
\theta(1,0)\left(b_{2}\right) & =\varphi(1,0) b_{2} \varphi(1,0)^{-1}=b_{1} a_{1} b_{2}\left(a_{2} b_{2} a_{2}^{-1}\right) b_{2}^{-1} a_{1}^{-1} b_{1}^{-1} \\
& \stackrel{(5)}{=} \quad b_{1} a_{1} b_{2} b_{2}^{-1} B^{-1} b_{2}^{2} b_{2}^{-1} a_{1}^{-1} b_{1}^{-1} \stackrel{(i v)}{=} b_{1}\left(a_{1} B a_{1}^{-1}\right)^{-1} b_{2} b_{1}^{-1} \\
& \stackrel{(3)}{=} \quad\left(b_{1} b_{1} b_{2}^{2}\right) b_{1} b_{2} b_{1}^{-1} \stackrel{(v)}{=} B b_{1} b_{2} b_{1}^{-1} \\
& \\
\theta(1,0)(B) & =l^{=}(1,0) B \varphi(1,0)^{-1}=b_{1} a_{1} b_{2}\left(a_{2} B a_{2}^{-1}\right) b_{2}^{-1} a_{1}^{-1} b_{1}^{-1} \\
& \stackrel{(6)}{=} b_{1} a_{1} b_{2} b_{2}^{-1} B^{-1} b_{2} b_{2}^{-1} a_{1}^{-1} b_{1}^{-1}=b_{1}\left(a_{1} B a_{1}^{-1}\right)^{-1} b_{1}^{-1} \\
& =b_{1} b_{1} b_{2}^{2} b_{1} b_{1}^{-1} \stackrel{(v)}{=} B
\end{aligned}
$$




$$
\begin{aligned}
& \theta(0,1)\left(b_{1}\right)=\varphi(0,1) b_{1} \varphi(0,1)^{-1}=a_{2}^{-1} b_{2}^{-1} b_{1} b_{2} a_{2} \\
& =\left(a_{2}^{-1} b_{2} a_{2}\right)^{-1}\left(a_{2}^{-1} b_{1} a_{2}\right)\left(a_{2}^{-1} b_{2} a_{2}\right) \\
& \stackrel{(10,11)}{=} b_{2}^{-1} B B^{-1} b_{2}^{-1} b_{1}^{-2} b_{2}^{-1} b_{1} b_{2} b_{1}^{2} b_{2} B B^{-1} b_{2} \\
& =\left(b_{2}^{-2} b_{1}^{-2}\right) b_{2}^{-1} b_{1} b_{2}\left(b_{1}^{2} b_{2}^{2}\right) \stackrel{(v)}{=} B^{-1} b_{2}^{-1} b_{1} b_{2} B \\
& \theta(0,1)\left(b_{2}\right)=\varphi(0,1) b_{2} \varphi(0,1)^{-1}=a_{2}^{-1} b_{2}^{-1} b_{2} b_{2} a_{2}=a_{2}^{-1} b_{2} a_{2} \stackrel{(11)}{=} B^{-1} b_{2} \\
& \theta(0,1)(B)=\varphi(0,1) B \varphi(0,1)^{-1}=a_{2}^{-1} b_{2}^{-1} B b_{2} a_{2} \\
& =\left(a_{2}^{-1} b_{2} a_{2}\right)^{-1}\left(a_{2}^{-1} B a_{2}\right)\left(a_{2}^{-1} b_{2} a_{2}\right) \\
& \stackrel{(11,12)}{=} b_{2}^{-1} B B^{-1} b_{2}^{-1} b_{1}^{-2} b_{2}^{-1} B B^{-1} b_{2} \\
& =b_{2}^{-2} b_{1}^{-2} \stackrel{(v)}{=} B^{-1} \text {. }
\end{aligned}
$$

Afim de simplificar as expressões de $\theta$, vamos fazer a seguinte mudança de base no grupo $F\left(b_{1}, b_{2}\right)$ :

$$
F(u, v) \rightleftarrows F\left(b_{1}, b_{2}\right)
$$

$$
\left\{\begin{array} { l } 
{ u \longmapsto b _ { 1 } b _ { 2 } } \\
{ v \longmapsto b _ { 2 } ^ { - 1 } . }
\end{array} \quad \left\{\begin{array}{l}
b_{1} \longmapsto u v \\
b_{2} \longmapsto v^{-1} .
\end{array}\right.\right.
$$

Notemos que o elemento $B=b_{1}^{2} b_{2}^{2}$ se escreve como $u v u v^{-1}$, o qual também denotaremos por $B$. Vamos calcular $\theta(1,0)$ e $\theta(0,1)$ nessa nova base.

$$
\begin{gathered}
\begin{aligned}
\theta(1,0)(u)= & \theta(1,0)\left(b_{1} b_{2}\right)=\theta(1,0)\left(b_{1}\right) \theta(1,0)\left(b_{2}\right) \\
= & B b_{1} b_{2}^{-1} b_{1}^{-1} b_{2}^{-1} b_{1}^{-1} B^{-1} B b_{1} b_{2} b_{1}^{-1} \\
= & B b_{1} b_{2}^{-1} b_{1}^{-2}=B b_{1} b_{2}\left(b_{2}^{-2} b_{1}^{-2}\right)=B b_{1} b_{2} B^{-1} \\
= & B u v v^{-1} B^{-1}=B u B^{-1}
\end{aligned} \\
\begin{aligned}
\theta(1,0)(v)=\theta(1,0)\left(b_{2}^{-1}\right)=\theta(1,0)\left(b_{2}\right)^{-1}=b_{1} b_{2}^{-1} b_{1}^{-1} B^{-1} \\
=u v v v^{-1} u^{-1} B^{-1}=u v u^{-1} B^{-1}=u v\left(u v^{-1} v u^{-1}\right) u^{-1} B^{-1} \\
=\left(u v u v^{-1}\right) v u^{-1} u^{-1} B^{-1}=B v u^{-2} B^{-1}
\end{aligned} \\
\begin{aligned}
\theta(0,1)(u) & =\theta(0,1)\left(b_{1} b_{2}\right)=\theta(0,1)\left(b_{1}\right) \theta(1,0)\left(b_{2}\right) \\
& =B^{-1} b_{2}^{-1} b_{1} b_{2} B B^{-1} b_{2}=B^{-1} b_{2}^{-1} b_{1}^{-1} b_{1}^{2} b_{2}^{2} \\
& =B^{-1} v v^{-1} u^{-1} B=B^{-1} u^{-1} B
\end{aligned} \\
\theta(0,1)(v)=\theta(0,1)\left(b_{2}^{-1}\right)=\theta(0,1)\left(b_{2}\right)^{-1}=b_{2}^{-1} B=v B .
\end{gathered}
$$


Afim de explicitar o automorfismo $\theta(m, n)$ para todo $(m, n) \in \mathbb{Z} \rtimes \mathbb{Z}$, vamos mostrar dois resultados preliminares.

Lema B.0.15. Para todo $(m, 0) \in \mathbb{Z} \rtimes \mathbb{Z}$, temos que

$$
\theta(m, 0):\left\{\begin{array}{l}
u \longmapsto B^{m} u B^{-m} \\
v \longmapsto B^{m} v u^{-2 m} B^{-m} \\
B \longmapsto B .
\end{array}\right.
$$

Demonstração. Para $m=0$ e $m=1$ o resultado é verdadeiro. Vamos proceder por indução em $m$. Suponhamos o resultado verdadeiro para $m \geq 1$. O símbolo $*$ significa que estamos usando a hipótese de indução. Temos que

$$
\begin{gathered}
\begin{aligned}
\theta(m+1,0)(u) & =\theta(m, 0)(\theta(1,0)(u))=\theta(m, 0)\left(B u B^{-1}\right) \\
& =\theta(m, 0)(B) \theta(m, 0)(u) \theta(m, 0)(B)^{-1} \\
& \stackrel{*}{=} B B^{m} u B^{-m} B^{-1}=B^{m+1} u B^{-(m+1)}
\end{aligned} \\
\begin{aligned}
& \theta(m+1,0)(v)= \theta(m, 0)(\theta(1,0)(v))=\theta(m, 0)\left(B v u^{-2} B^{-1}\right) \\
&= \theta(m, 0)(B) \theta(m, 0)(v) \theta(m, 0)(u)^{-2} \theta(m, 0)(B)^{-1} \\
& \stackrel{*}{=} B B^{m} v u^{-2 m} B^{-m}\left(B^{m} u B^{-m}\right)^{-2} B^{-1} \\
&= B^{m+1} v u^{-2(m+1)} B^{-(m+1)} \\
& \theta(m+1,0)(B)=\theta(m, 0)(\theta(1,0)(B))=\theta(m, 0)(B) \stackrel{*}{=} B .
\end{aligned}
\end{gathered}
$$

Se $m<0$, então $-m>0$ e já sabemos $\theta(-m, 0)$. Assim, temos

$$
B=\theta(0,0)(B)=\theta(m, 0)(\theta(-m, 0)(B))=\theta(m, 0)(B)
$$

Temos também

$$
\begin{aligned}
u & =\theta(m, 0)(\theta(-m, 0)(u))=\theta(m, 0)\left(B^{-m} u B^{m}\right) \\
& =\theta(m, 0)(B)^{-m} \theta(m, 0)(u) \theta(m, 0)(B)^{m} \\
& =B^{-m} \theta(m, 0)(u) B^{m}
\end{aligned}
$$

e

$$
\begin{aligned}
v & =\theta(m, 0)(\theta(-m, 0)(v))=\theta(m, 0)\left(B^{-m} v u^{2 m} B^{m}\right) \\
& =\theta(m, 0)(B)^{-m} \theta(m, 0)(v) \theta(m, 0)(u)^{2 m} \theta(m, 0)(B)^{m} \\
& =B^{-m} \theta(m, 0)(v)\left(B^{m} u B^{-m}\right)^{2 m} B^{m} \\
& =B^{-m} \theta(m, 0)(v) B^{m} u^{2 m},
\end{aligned}
$$

e portanto, segue que $\theta(m, 0)(u)=B^{m} u B^{-m}$ e $\theta(m, 0)(v)=B^{m} v u^{-2 m} B^{-m}$.

Lema B.0.16. Para todo $(0, n) \in \mathbb{Z} \rtimes \mathbb{Z}$, temos que

$$
\theta(0, n):\left\{\begin{array}{l}
u \longmapsto B^{-\delta_{n}} u^{(-1)^{n}} B^{\delta_{n}} \\
v \longmapsto v B^{\delta_{n}} \\
B \longmapsto B^{(-1)^{n}}
\end{array}\right.
$$


sendo $\delta_{n}=\left\{\begin{array}{l}0, \text { se } n \text { é par } \\ 1, \text { se } n \text { é ímpar. }\end{array}\right.$

Demonstração. Para $n=0$ e $n=1$ o resultado é verdadeiro. Vamos proceder por indução em $n$. Suponhamos o resultado verdadeiro para $n \geq 1$. O símbolo $*$ significa que estamos usando a hipótese de indução. Assim, temos que

$$
\begin{aligned}
\theta(0, n+1)(u) & =\theta(0,1)(\theta(0, n)(u)) \stackrel{*}{=} \theta(0,1)\left(B^{-\delta_{n}} u^{(-1)^{n}} B^{\delta_{n}}\right) \\
& =\theta(0,1)(B)^{-\delta_{n}} \theta(0,1)(u)^{(-1)^{n}} \theta(0,1)(B)^{\delta_{n}} \\
& =\left(B^{-1}\right)^{-\delta_{n}}\left(B^{-1} u^{-1} B\right)^{(-1)^{n}}\left(B^{-1}\right)^{\delta_{n}} \\
& =B^{\delta_{n}} B^{-1} u^{(-1)^{n+1}} B B^{-\delta_{n}}=B^{-\delta_{(n+1)}} u^{(-1)^{n+1}} B^{\delta_{(n+1)}} \\
\theta(0, n+1)(v) & =\theta(0,1)(\theta(0, n)(v)) \stackrel{*}{=} \theta(0,1)\left(v B^{\delta_{n}}\right) \\
& =\theta(0,1)(v) \theta(0,1)(B)^{\delta_{n}}=v B\left(B^{-1}\right)^{\delta_{n}} \\
& =v B^{\delta_{(n+1)}} \\
\theta(0, n+1)(B)= & \theta(0,1)(\theta(0, n)(B)) \stackrel{*}{=} \theta(0,1)\left(B^{(-1)^{n}}\right) \\
= & \theta(0,1)(B)^{(-1)^{n}}=\left(B^{-1}\right)^{(-1)^{n}}=B^{(-1)^{(n+1)}} .
\end{aligned}
$$

Se $n<0$, então $-n>0$ e já sabemos $\theta(0,-n)$. Assim, temos que

$$
B=\theta(0,0)(B)=\theta(0, n)(\theta(0,-n)(B))=\theta(0, n)\left(B^{(-1)^{-n}}\right)=\theta(0, n)(B)^{(-1)^{n}},
$$

e portanto, segue que $\theta(0, n)(B)=B^{(-1)^{n}}$. Temos também que

$$
u=\theta(0, n)(\theta(0,-n)(u))=\theta(0, n)\left(B^{\delta_{-n}} u^{(-1)^{-n}} B^{-\delta_{n}}\right)
$$

e

$$
\begin{aligned}
v & =\theta(0, n)(\theta(0,-n)(v))=\theta(0, n)\left(v B^{\delta_{-n}}\right) \\
& =\theta(0, n)(v) \theta(0, n)(B)^{\delta_{n}}=\theta(0, n)(v)\left(B^{(-1)^{n}}\right)^{\delta_{n}} .
\end{aligned}
$$

Se $n$ é par, então $u=\theta(0, n)(u)$ e $v=\theta(0, n)(v)$ e portanto, para este caso, valem as fórmulas. Se $n$ é ímpar, então temos que

$$
\begin{aligned}
u & =\theta(0, n)\left(B^{-1} u^{-1} B\right) \\
& =\theta(0, n)(B)^{-1} \theta(0, n)(u)^{-1} \theta(0, n)(B) \\
& =\left(B^{-1}\right)^{-1} \theta(0, n)(u)^{-1} B^{-1}
\end{aligned}
$$

e

$$
v=\theta(0, n)(v) B,
$$

e portanto, temos que $\theta(0, n)=B^{-1} u^{-1} B$ e $\theta(0, n)=v B$. Logo, valem as fórmulas para este caso, o que encerra a demonstração.

Vamos agora explicitar o automorfismo $\theta(m, n)$ e o isomorfismo entre $F(u, v) \rtimes_{\theta}(\mathbb{Z} \rtimes \mathbb{Z})$ e $P_{2}\left(\mathbb{K}^{2}\right)$.

Para não carregar a notação, nós denotaremos um elemento $(w,(m, n)) \in F(u, v) \rtimes_{\theta}(\mathbb{Z} \rtimes \mathbb{Z})$ por $(w ; m, n)$. Para evitar confusão, denotaremos por $\mathbb{1}$ o elemento neutro de $F(u, v)$ 
Teorema B.0.17. O homomorfismo $\theta: \mathbb{Z} \rtimes \mathbb{Z} \rightarrow \operatorname{Aut}(F(u, v))$ é definido por:

$$
\theta(m, n):\left\{\begin{array}{l}
u \longmapsto B^{m-\delta_{n}} u^{(-1)^{n}} B^{-m+\delta_{n}} \\
v \longmapsto B^{m} v u^{-2 m} B^{-m+\delta_{n}} \\
B \longmapsto B^{(-1)^{n}}
\end{array} \quad \delta_{n}=\left\{\begin{array}{l}
0, \text { se } n \text { é par; } \\
1, \text { se n é ímpar. }
\end{array}\right.\right.
$$

Os isomorfismos

$$
F(u, v) \rtimes_{\theta}(\mathbb{Z} \rtimes \mathbb{Z}) \underset{\gamma}{\stackrel{\lambda}{\rightleftarrows}} P_{2}\left(\mathbb{K}^{2}\right)
$$

são definidos por

$$
\lambda:\left\{\begin{array}{l}
(u ; 0,0) \longmapsto b_{1} b_{2} \\
(v ; 0,0) \longmapsto b_{2}^{-1} \\
(B ; 0,0) \longmapsto B \\
(\mathbb{1} ; 1,0) \longmapsto b_{1} a_{1} b_{2} a_{2} \\
(\mathbb{1} ; 0,1) \longmapsto a_{2}^{-1} b_{2}^{-1}
\end{array} \quad \gamma:\left\{\begin{array}{l}
a_{1} \longmapsto\left(v^{-1} u^{-1} ; 1,1\right) \\
a_{2} \longmapsto(v ; 0,-1) \\
b_{1} \longmapsto(u v ; 0,0) \\
b_{2} \longmapsto\left(v^{-1} ; 0,0\right) \\
B \longmapsto(B ; 0,0) .
\end{array}\right.\right.
$$

Demonstração. Vamos usar os Lemas B.0.15 e B.0.16 para explicitar o homorfismo $\theta(m, n): F(u, v) \rightarrow F(u, v)$. Assim, temos que

$$
\begin{aligned}
& \theta(m, n)(B)=\theta(m, 0) \circ \theta(0, n)(B)=\theta(m, 0)\left(B^{(-1)^{n}}\right)=\theta(m, 0)(B)^{(-1)^{n}}=B^{(-1)^{n}} \\
& \begin{aligned}
\theta(m, n)(u)= & \theta(m, 0) \circ \theta(0, n)(u)=\theta(m, 0)\left(B^{-\delta_{n}} u^{(-1)^{n}} B^{\delta_{n}}\right) \\
= & \theta(m, 0)(B)^{-\delta_{n}} \theta(m, 0)(u)^{(-1)^{n}} \theta(m, 0)(B)^{\delta_{n}} \\
= & B^{-\delta_{n}}\left(B^{m} u B^{-m}\right)^{(-1)^{n}} B^{\delta_{n}} \\
= & B^{m-\delta_{n}} u^{(-1)^{n}} B^{-m \delta_{n}} \\
& \\
\theta(m, n)(v) & =\theta(m, 0) \circ \theta(0, n)(v)=\theta(m, 0)\left(v B^{\delta_{n}}\right) \\
& =\theta(m, 0)(v) \theta(m, 0)(B)^{\delta_{n}} \\
& =B^{m} v u^{-2 m} B^{-m} B^{\delta_{n}} \\
& =B^{m} v u^{-2 m} B^{-m \delta_{n}}
\end{aligned}
\end{aligned}
$$

Agora vamos explicitar os valores dos isomorfismos $\lambda$ e $\gamma$.

$$
\begin{gathered}
\lambda(u ; 0,0)=\lambda\left(b_{1} b_{2} ; 0,0\right)=b_{1} b_{2} \varphi(0,0)=b_{1} b_{2} \\
\lambda(v ; 0,0)=\lambda\left(b_{2}^{-1} ; 0,0\right)=b_{2}^{-1} \varphi(0,0)=b_{2}^{-1} \\
\lambda(B ; 0,0)=\lambda\left(u v u v^{-1} ; 0,0\right)=\lambda\left(b_{1}^{2} b_{2}^{2},(0,0)\right)=b_{1}^{2} b_{2}^{2} \varphi(0,0)=B \\
\lambda(\mathbb{1} ; 1,0)=1 \varphi(1,0)=b_{1} a_{1} b_{2} a_{2} \\
\lambda(\mathbb{1} ; 0,1)=1 \varphi(0,1)=a_{2}^{-1} b_{2}^{-1}
\end{gathered}
$$




$$
\begin{aligned}
& \gamma\left(a_{1}\right)=\left(a_{1} \varphi\left(f\left(a_{1}^{-1}\right)\right) ; f\left(a_{1}\right)\right)=\left(a_{1} \varphi(1,1)^{-1} ; 1,1\right)=\left(a_{1}(\varphi(1,0) \varphi(0,1))^{-1} ; 1,1\right) \\
&=\left(a_{1}\left(b_{1} a_{1} b_{2} a_{2} a_{2}^{-1} b_{2}^{-1}\right)^{-1} ; 1,1\right)=\left(b_{1}^{-1} ; 1,1\right)=\left(v^{-1} u^{-1} ; 1,1\right) \\
& \gamma\left(a_{2}\right)=\left(a_{2} \varphi\left(f\left(a_{2}^{-1}\right)\right) ; f\left(a_{2}\right)\right)=\left(a_{2} \varphi(0,-1)^{-1} ; 0,-1\right)=\left(a_{2} \varphi(0,1) ; 0,-1\right) \\
&=\left(a_{2} a_{2}^{-1} b_{2}^{-1} ; 0,-1\right)=\left(b_{2}^{-1} ; 0,-1\right)=(v ; 0,-1) \\
& \gamma\left(b_{1}\right)=\left(b_{1} \varphi\left(f\left(b_{1}^{-1}\right)\right) ; f\left(b_{1}\right)\right)=\gamma\left(b_{1} \varphi(0,0) ; 0,0\right)=\gamma(u v ; 0,0) \\
& \gamma\left(b_{2}\right)=\left(b_{2} \varphi\left(f\left(b_{2}^{-1}\right)\right) ; f\left(b_{2}\right)\right)=\gamma\left(b_{2} \varphi(0,0) ; 0,0\right)=\gamma\left(v^{-1} ; 0,0\right) .
\end{aligned}
$$

Vamos calcular $f \circ \lambda$ nos geradores de $F(u, v) \rtimes_{\theta}(\mathbb{Z} \rtimes \mathbb{Z})$. Temos que

$$
\begin{gathered}
(f \circ \lambda)(u ; 0,0)=f\left(b_{1} b_{2}\right)=(0,0) \\
(f \circ \lambda)(v ; 0,0)=f\left(b_{2}^{-1}\right)=(0,0) \\
(f \circ \lambda)(\mathbb{1} ; 1,0)=f\left(b_{1} a_{1} b_{2} a_{2}\right)=(0,0)(1,1)(0,0)(0,-1)=(1,0) \\
(f \circ \lambda)(\mathbb{1} ; 0,1)=f\left(a_{2}^{-1} b_{2}^{-1}\right)=(0,-1)^{-1}(0,0)=(0,1) .
\end{gathered}
$$

Geometricamente, é fácil ver que $r$ é induzido do homeomorfismo descrito nas figuras B.1, B.2, B.3 e B.4. Assim, abusaremos da notação e denotaremos $f \circ \lambda$ por $\left(p_{1}\right)_{\#}$. Deste modo, resumimos as informações de $P_{2}\left(\mathbb{K}^{2}\right)$ até aqui encontradas na seguinte observação:

Observação B.0.18 (Presentação de $P_{2}\left(\mathbb{K}^{2}\right)$ ). A menos de isomorfismo, $P_{2}\left(\mathbb{K}^{2}\right)$ se escreve na forma $F(u, v) \rtimes_{\theta}(\mathbb{Z} \rtimes \mathbb{Z})$, sendo $F(u, v)$ o grupo livre gerado por $\{u, v\}$ e $\theta: \mathbb{Z} \rtimes \mathbb{Z} \rightarrow \operatorname{Aut}(F(u, v))$ definida do seguinte modo:

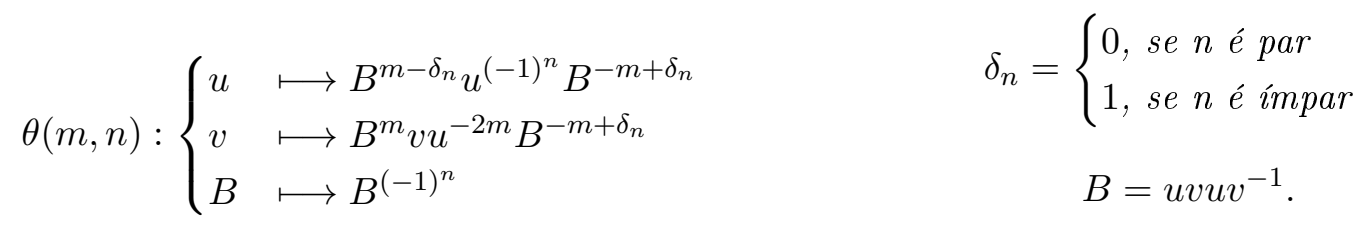

Com respeito a esta escrita, temos que $\left(p_{1}\right)_{\#}: P_{2}\left(\mathbb{K}^{2}\right) \rightarrow \pi_{1}\left(\mathbb{K}^{2}\right)=\mathbb{Z} \rtimes \mathbb{Z}$ é a projeção de $F(u, v) \rtimes_{\theta}(\mathbb{Z} \rtimes \mathbb{Z})$ em $\mathbb{Z} \rtimes \mathbb{Z}$, isto é, $\left(p_{1}\right)_{\#}(w ; r, s)=(r, s)$.

Vamos agora estudar como a conjugação por $\sigma$ em $P_{2}\left(\mathbb{K}^{2}\right)$ se comporta em $F(u, v) \rtimes_{\theta}(\mathbb{Z} \rtimes \mathbb{Z})$. Comecemos definindo a notação. Seja

$$
\begin{aligned}
c_{\sigma}: P_{2}\left(\mathbb{K}^{2}\right) & \longrightarrow P_{2}\left(\mathbb{K}^{2}\right) \\
x & \longmapsto \sigma x \sigma^{-1} .
\end{aligned}
$$

Temos pela presentação de $P_{2}\left(\mathbb{K}^{2}\right)$ que

$$
\begin{array}{lll}
c_{\sigma}\left(a_{1}\right)=b_{1} & c_{\sigma}\left(b_{1}\right)=B a_{1} B^{-1} & c_{\sigma}(B)=B . \\
c_{\sigma}\left(a_{2}\right)=b_{2} & c_{\sigma}\left(b_{2}\right)=B a_{2} B^{-1} &
\end{array}
$$


Seja agora $l_{\sigma}: F(u, v) \rtimes_{\theta}(\mathbb{Z} \rtimes \mathbb{Z}) \rightarrow F(u, v) \rtimes_{\theta}(\mathbb{Z} \rtimes \mathbb{Z})$ definido de modo que o seguinte diagrama seja comutativo:

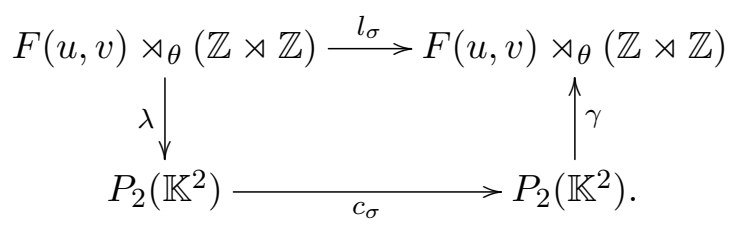

Vamos calcular $l_{\sigma}$ nos geradores de $F(u, v) \rtimes_{\theta}(\mathbb{Z} \rtimes \mathbb{Z})$. Temos que

$$
\begin{aligned}
& l_{\sigma}(u ; 0,0)=\left(\gamma \circ c_{\sigma} \circ \lambda\right)(u ; 0,0)=\left(\gamma \circ c_{\sigma}\right)\left(b_{1} b_{2}\right)=\gamma\left(B a_{1} B^{-1} B a_{2} B^{-1}\right) \\
& =\gamma(B) \gamma\left(a_{1}\right) \gamma\left(a_{2}\right) \gamma(B)^{-1}=(B ; 0,0)\left(v^{-1} u^{-1} ; 1,1\right)(v ; 0,-1)(B, 0,0)^{-1} \\
& =\left(B v^{-1} u^{-1} \theta(1,1)(v) \theta(1,0)\left(B^{-1}\right) ; 1,0\right)=\left(B v^{-1} u^{-1} B v u^{-2} B^{-1} ; 1,0\right) \\
& =\left(B v^{-1} u^{-1} u v u v^{-1} v u^{-2} B^{-1} ; 1,0\right)=\left(B u^{-1} B^{-1} ; 1,0\right) \\
& l_{\sigma}(v ; 0,0)=\left(\gamma \circ c_{\sigma} \circ \lambda\right)(v ; 0,0)=\left(\gamma \circ c_{\sigma}\right)\left(b_{2}^{-1}\right)=\gamma\left(B a_{2}^{-1} B^{-1}\right) \\
& =\gamma(B) \gamma\left(a_{2}\right)^{-1} \gamma(B)^{-1}=(B ; 0,0)(v ; 0,-1)^{-1}(B ; 0,0)^{-1} \\
& =(B ; 0,0)\left(\theta(0,1)(v)^{-1} ; 0,1\right)\left(B^{-1} ; 0,0\right)=\left(B(v B)^{-1} \theta(0,1)(B)^{-1} ; 0,1\right) \\
& =\left(B B^{-1} v^{-1} B ; 0,1\right)=\left(v^{-1} B ; 0,1\right) \\
& l_{\sigma}(B, 0,0)=\left(\gamma \circ c_{\sigma} \circ \lambda\right)(B, 0,0)=\left(\gamma \circ c_{\sigma}\right)(B)=\gamma(B)=(B ; 0,0) \\
& l_{\sigma}(\mathbb{1} ; 1,0)=\left(\gamma \circ c_{\sigma} \circ \lambda\right)(\mathbb{1} ; 1,0)=\left(\gamma \circ c_{\sigma}\right)\left(b_{1} a_{1} b_{2} a_{2}\right)=\gamma\left(B a_{1} B^{-1} b_{1} B a_{2} B^{-1} b_{2}\right) \\
& =\gamma(B) \gamma\left(a_{1}\right) \gamma(B)^{-1} \gamma\left(b_{1}\right) \gamma(B) \gamma\left(a_{2}\right) \gamma(B)^{-1} \gamma\left(b_{2}\right) \\
& =(B ; 0,0)\left(v^{-1} u^{-1} ; 1,1\right)(B ; 0,0)^{-1}(u v ; 0,0)(B ; 0,0)(v ; 0,-1)(B ; 0,0)^{-1}\left(v^{-1} ; 0,0\right) \\
& =\left(B v^{-1} u^{-1} \theta(1,1)(B)^{-1} \theta(1,1)(u) \theta(1,1)(v) \theta(1,1)(B) \theta(1,1)(v) \theta(1,0)(B)^{-1} \theta(1,0)(v)^{-1} ; 1,0\right) \\
& =\left(B v^{-1} u^{-1} B u^{-1} B v u^{-2} B^{-1} B v u^{-2} B^{-1}\left(B v u^{-2} B^{-1}\right)^{-1} ; 1,0\right) \\
& =\left(B v^{-1} u^{-1} B u^{-1} B v u^{-2} B^{-1} B v u^{-2} B^{-1} B u^{2} v^{-1} B^{-1} ; 1,0\right) \\
& =\left(B v^{-1} u^{-1} B u^{-1} B v u^{-2} B^{-1} ; 1,0\right) \\
& =\left(u v u v^{-1} v^{-1} u^{-1} u v u v^{-1} u^{-1} u v u v^{-1} v u^{-2} v u^{-1} v^{-1} u^{-1} ; 1,0\right)=(\mathbb{1} ; 1,0) \\
& l_{\sigma}(\mathbb{1} ; 0,1)=\left(\gamma \circ c_{\sigma} \circ \lambda\right)(\mathbb{1} ; 0,1)=\left(\gamma \circ c_{\sigma}\right)\left(a_{2}^{-1} b_{2}^{-1}\right) \\
& =\gamma\left(b_{2}^{-1} B a_{2} B^{-1}\right)=\gamma\left(b_{2}\right)^{-1} \gamma(B) \gamma\left(a_{2}\right) \gamma(B)^{-1} \\
& =\left(v^{-1} ; 0,0\right)^{-1}(B ; 0,0)(v ; 0,-1)^{-1}(B ; 0,0)^{-1} \\
& =(v ; 0,0)(B ; 0,0)\left(\theta(0,1)(v)^{-1} ; 0,1\right)\left(B^{-1} ; 0,0\right) \\
& =\left(v B(v B)^{-1} \theta(0,1)(B)^{-1} ; 0,1\right)=(B ; 0,1)
\end{aligned}
$$

Vamos agora explicitar o automorfismo $l_{\sigma}$ em potências dos geradores de $F(u, v) \rtimes_{\theta}(\mathbb{Z} \rtimes \mathbb{Z})$. Mais precisamente, vamos mostrar o seguinte resultado:

Lema B.0.19. Para cada $r, s, m, n \in \mathbb{Z}$, valem as seguintes expressões para o automorfismo $l_{\sigma}$ : 

1. $l_{\sigma}\left(u^{r} ; 0,0\right)=\left(\left(B u^{-1}\right)^{r} B^{-r} ; r, 0\right)$;
2. $l_{\sigma}\left(v^{s} ; 0,0\right)=\left((u v)^{-s}(u B)^{\delta_{s}} ; 0, s\right)$;
3. $l_{\sigma}(\mathbb{1} ; m, 0)=(\mathbb{1} ; m, 0)$;
4. $l_{\sigma}(\mathbb{1} ; 0, n)=\left(B^{\delta_{n}} ; 0, n\right)$.

Demonstração. Primeiramente vamos mostrar que $l_{\sigma}\left(u^{r}, 0,0\right)=\left(\left(B u^{-1}\right)^{r} B^{-r}, r, 0\right)$. Vamos proceder por indução em $r$. Para $r=0$ é óbvio e já mostramos para $r=1$. Suponhamos o resultado verdadeiro para $r \geq 1$. O símbolo $*$ significa que estamos usando a hipótese de indução.

$$
\begin{aligned}
l_{\sigma}\left(u^{r+1} ; 0,0\right) & =l_{\sigma}(u ; 0,0) l_{\sigma}\left(u^{r} ; 0,0\right) \stackrel{*}{=}\left(B u^{-1} B^{-1} ; 1,0\right)\left(\left(B u^{-1}\right)^{r} B^{-r} ; r, 0\right) \\
& =\left(B u^{-1} B^{-1}\left(\theta(1,0)(B) \theta(1,0)(u)^{-1}\right)^{r} \theta(1,0)(B)^{-r} ; r+1,0\right) \\
& =\left(B u^{-1} B^{-1}\left(B\left(B u B^{-1}\right)^{-1}\right)^{r} B^{-r} ; r+1,0\right) \\
& =\left(B u^{-1} B^{-1}\left(B B u^{-1} B^{-1}\right)^{r} B^{-r} ; r+1,0\right) \\
& =\left(B u^{-1} B^{-1} B\left(B u^{-1}\right)^{r} B^{-1} B^{-r} ; r+1,0\right) \\
& =\left(\left(B u^{-1}\right)^{r+1} B^{-(r+1)} ; r, 0\right) .
\end{aligned}
$$

Se $r<0$, então $-r>0$ e já sabemos $l_{\sigma}\left(u^{-r} ; 0,0\right)$. Assim, temos que

$$
\begin{aligned}
l_{\sigma}\left(u^{r} ; 0,0\right) & =l_{\sigma}\left(u^{-r} ; 0,0\right)^{-1}=\left(\left(B u^{-1}\right)^{-r} B^{r} ;-r, 0\right)^{-1}=\left(\left(\theta(r, 0)\left(\left(B u^{-1}\right)^{-r} B^{r}\right)\right)^{-1} ; r, 0\right) \\
& =\left(\left(\left(\theta(r, 0)(B) \theta(r, 0)(u)^{-1}\right)^{-r} \theta(r, 0)(B)^{r}\right)^{-1} ; r, 0\right) \\
& =\left(\left(\left(B\left(B^{r} u B^{-r}\right)^{-1}\right)^{-r} B^{r}\right)^{-1} ; r, 0\right)=\left(\left(\left(B B^{r} u^{-1} B^{-r}\right)^{-r} B^{r}\right)^{-1} ; r, 0\right) \\
& =\left(\left(B^{r}\left(B u^{-1}\right)^{-r} B^{-r} B^{r}\right)^{-1} ; r, 0\right)=\left(\left(B u^{-1}\right)^{r} B^{-r} ; r, 0\right) .
\end{aligned}
$$

Agora vamos mostrar que $l_{\sigma}\left(v^{s}, 0,0\right)=\left((u v)^{-s}(u B)^{\delta_{s}} ; 0, s\right)$. Primeiramente suponhamos $s$ par. Neste caso temos que $s=2 k$, para algum $k \in \mathbb{Z}$, e queremos mostrar que

$$
l_{\sigma}\left(v^{2 k} ; 0,0\right)=\left((u v)^{-2 k} ; 0,2 k\right) .
$$

Para $k=1$, temos que

$$
\begin{aligned}
l_{\sigma}\left(v^{2} ; 0,0\right) & =l_{\sigma}(v ; 0,0) l_{\sigma}(v ; 0,0)=\left(v^{-1} B ; 0,1\right)\left(v^{-1} B ; 0,1\right) \\
& =\left(v^{-1} B \theta(0,1)(v)^{-1} \theta(0,1)(B) ; 0,2\right)=\left(\left(v^{-1} B(v B)^{-1} B^{-1} ; 0,2\right)\right. \\
& =\left(v^{-2} B^{-1} ; 0,2\right)=\left(v^{-2} v u^{-1} v^{-1} u^{-1} ; 0,2\right)=\left((u v)^{-2} ; 0,2\right) .
\end{aligned}
$$

Portanto, para $k=0$ e $k=1$ a fórmula é verdadeira. Vamos proceder por indução em $k$. Suponhamos o resultado verdadeiro para algum $k \geq 1$. O símbolo $*$ significa que estamos usando a hipótese de indução. Assim, temos que

$$
\begin{aligned}
l_{\sigma}\left(v^{2(k+1)} ; 0,0\right) & =l_{\sigma}\left(v^{2 k} ; 0,0\right) l_{\sigma}\left(v^{2} ; 0,0\right) \stackrel{*}{=}\left((u v)^{-2 k} ; 0,2 k\right)\left((u v)^{-2} ; 0,2\right) \\
& =\left((u v)^{-2 k}(\theta(0,2 k)(u) \theta(0,2 k)(v))^{-2} ; 0,2 k+2\right) \\
& =\left((u v)^{-2 k}(u v)^{-2} ; 0,2(k+1)\right)=\left((u v)^{-2(k+1)} ; 0,2(k+1)\right) .
\end{aligned}
$$

Se $k<0$, então $-k>0$, e já sabemos $l_{\sigma}\left(v^{-2 k}, 0,0\right)$. Assim, temos

$$
\begin{aligned}
l_{\sigma}\left(v^{2 k} ; 0,0\right) & =l_{\sigma}\left(v^{-2 k} ; 0,0\right)^{-1}=\left((u v)^{2 k} ; 0,-2 k\right)^{-1}=\left(\left(\theta(0,2 k)\left((u v)^{2 k}\right)\right)^{-1} ; 0,2 k\right) \\
& =\left(\left((\theta(0,2 k)(u) \theta(0,2 k)(v))^{2 k}\right)^{-1} ; 0,2 k\right)=\left((u v)^{-2 k} ; 0,2 k\right) .
\end{aligned}
$$


Agora suponhamos $s$ ímpar. Seja $k \in \mathbb{Z}$ tal que $s=2 k+1$. Queremos mostrar que

$$
l_{\sigma}\left(v^{2 k+1} ; 0,0\right)=\left((u v)^{-2 k-1} u B ; 0,2 k+1\right) .
$$

Já sabemos a fórmula para $l_{\sigma}\left(v^{2 k} ; 0,0\right)$. Assim, temos que

$$
\begin{aligned}
l_{\sigma}\left(v^{2 k+1} ; 0,0\right) & =l_{\sigma}\left(v^{2 k} ; 0,0\right) l_{\sigma}(v ; 0,0)=\left((u v)^{-2 k} ; 0,2 k\right)\left(v^{-1} B ; 0,1\right) \\
& =\left((u v)^{-2 k} \theta(0,2 k)(v)^{-1} \theta(0,2 k)(B) ; 0,2 k+1\right)=\left((u v)^{-2 k} v^{-1} B ; 0,2 k+1\right) \\
& =\left((u v)^{-2 k}(u v)^{-1}(u v) v^{-1} B ; 0,2 k+1\right)=\left((u v)^{-2 k-1} u B, 0,2 k+1\right) .
\end{aligned}
$$

A fórmula 3 decorre facilmente, pois temos que

$$
l_{\sigma}(\mathbb{1} ; m, 0)=l_{\sigma}(\mathbb{1} ; 1,0)^{m}=(\mathbb{1} ; 1,0)^{m}=(\mathbb{1} ; m, 0) .
$$

Para mostra a fórmula 4 , notemos que para cada $n \in \mathbb{Z}$, existe um único $k \in \mathbb{Z}$ tal que $n=2 k+\delta_{n}$. Assim, temos que

$$
\begin{aligned}
l_{\sigma}(\mathbb{1} ; 0, n) & =l_{\sigma}(\mathbb{1} ; 0,1)^{n}=(B ; 0,1)^{n}=(B ; 0,1)^{2 k}(B ; 0,1)^{\delta_{n}} \\
& =((B ; 0,1)(B ; 0,1))^{k}\left(B^{\delta_{n}} ; 0, \delta_{n}\right) \\
& =(B \theta(0,1)(B) ; 0,2)^{k}\left(B^{\delta_{n}}, 0, \delta_{n}\right) \\
& =\left(B B^{-1} ; 0,2\right)^{k}\left(B^{\delta_{n}} ; 0, \delta_{n}\right)=(\mathbb{1} ; 0,2 k)\left(B^{\delta_{n}} ; 0, \delta_{n}\right) \\
& =\left(\theta(0,2 n)(B)^{\delta_{n}} ; 0,2 k+\delta_{n}\right)=\left(B^{\delta_{n}} ; 0, n\right) .
\end{aligned}
$$

Seja $g: F(u, v) \rightarrow \mathbb{Z} \rtimes \mathbb{Z}$ definida nos geradores por

$$
g(u)=(1,0) \quad \text { e } \quad g(v)=(0,1) .
$$

Notemos que pelo Lema B.0.19, o seguinte diagrama é comutativo:

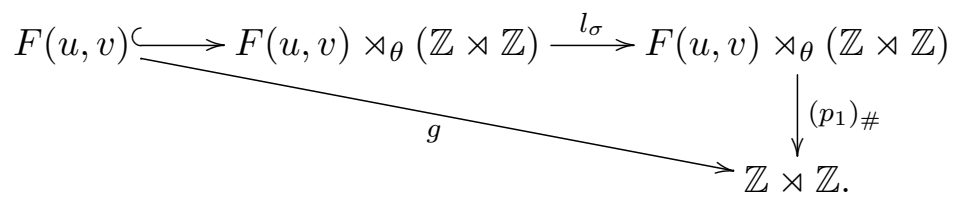

Notemos ainda que se $w \in F(u, v)$ e $g(w)=(r, s)$, então $v^{-s} u^{-r} w \in \operatorname{ker} g$. Logo, $w=u^{r} v^{s} x$ para algum $x \in \operatorname{ker} g$. Por estas observações, vamos obter uma presentação para o grupo ker $g$ utilizando o método de Reidemeister-Schreier, que também se aplica no caso de um subgrupo de índice não finito.

Afirmamos que um sistema de Schreier de ker $g$ é $S=\left\{v^{s} u^{r}\right\}_{r, s \in \mathbb{Z}}$. De fato, temos que

$$
g\left(v^{s} u^{r}\right)=(0,1)^{s}(1,0)^{r}=(0, s)(r, 0)=\left(r(-1)^{s}, s\right),
$$

o que implica que $\left.g\right|_{S}: S \rightarrow \mathbb{Z} \rtimes \mathbb{Z}$ é uma bijeção. Afim de facilitar o cálculo dos geradores de ker $g$, notemos que

$$
\begin{aligned}
g\left(v^{s} u^{r} v\right) & =(0,1)^{s}(1,0)^{r}(0,1)=(0, s)(r, 0)(0,1)=\left(r(-1)^{s}, s+1\right) \\
& =\left((-r)(-1)^{s+1}, s+1\right)=(0, s+1)(-r, 0)=(0,1)^{s+1}(1,0)^{-r}=g\left(v^{s+1} u^{-r}\right)
\end{aligned}
$$

Calculemos os geradores: 


$$
\begin{aligned}
& v^{s} u^{r} u{\overline{v^{s} u^{r} u}}^{-1}=v^{s} u^{r} u{\overline{v^{s} u^{r+1}}}^{-1}=v^{s} u^{r} u\left(v^{s} u^{r+1}\right)^{-1}=1 \\
& v^{s} u^{r} v{\overline{v^{s} u^{r} v}}^{-1}=v^{s} u^{r} v\left(v^{s+1} u^{-r}\right)^{-1}=v^{s} u^{r} v u^{r} v^{-1} v^{-s}=: \Gamma_{s, r} .
\end{aligned}
$$

Notemos que $\Gamma_{s, r}=\mathbb{1} \Leftrightarrow r=0$. Uma vez que $F(u, v)$ é livremente gerado por $\{u, v\}$, segue pelo método de Reidemeister-Schreier que ker $g$ é livremente gerado pelo conjunto $\left\{\Gamma_{s, r}\right\}_{s, r \in \mathbb{Z}, r \neq 0}$.

Seja agora $B_{s, r}:=v^{s} u^{r} B u^{-r} v^{-s} \in F(u, v)$. Notemos que $B_{s, r} \in \operatorname{ker} g$ e que $B_{0,0}=B$. Vamos

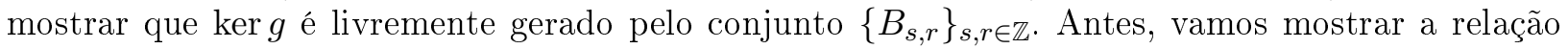
entre $\Gamma_{s, r}$ e $B_{s, r}$.

Lema B.0.20. Em ker $g$ valem as seguintes relaçôes:

1. $\Gamma s, r=\left\{\begin{array}{l}\prod_{i=0}^{r-1} B_{s, r-1-i}, \text { se } r \geq 1 \\ \prod_{i=0}^{-r-1} B_{s, r+i}^{-1}, \text { se } r \leq-1\end{array}\right.$

2. $B_{s, r}=\Gamma_{s, r+1} \Gamma_{s, r}^{-1}$, sendo $\Gamma_{s, 0}=\mathbb{1}$.

Demonstração. Primeiro vamos mostrar que o item 1 do enunciado do Lema é verdadeiro para $s=0$. Temos que

$$
\Gamma_{0,1}=u v u v^{-1}=B_{0,0}
$$

Suponhamos por indução que a fórmula do item 1 é verdadeira para algum $r \geq 1$ e $s=0$ e vamos mostrar que a fórmula é verdadeira para $r+1$. O símbolo $*$ significa que estamos usando a hipótese de indução.

$$
\begin{aligned}
\Gamma_{0, r+1} & =u^{r+1} v u^{r+1} v^{-1}=u u^{r} v u^{r}\left(v^{-1} u^{-1} u v\right) u v^{-1} \\
& =u\left(u^{r} v u^{r} v^{-1}\right) u^{-1}\left(u v u v^{-1}\right) \\
& =u \Gamma_{0, r} u^{-1} \Gamma_{0,1} \stackrel{*}{=} u\left(\prod_{i=0}^{r-1} B_{0, r-1-i}\right) u^{-1} B_{0,0} \\
& =\left(\prod_{i=0}^{r-1} B_{0, r-i}\right) B_{0,0}=\prod_{i=0}^{(r+1)-1} B_{0,(r+1)-1-i} .
\end{aligned}
$$

Agora temos que

$$
\Gamma_{0,-1}=u^{-1} v u^{-1} v^{-1}=u^{-1} v u^{-1} v^{-1}\left(u^{-1} u\right)=u^{-1}\left(u v u v^{-1}\right)^{-1} u=u^{-1} B_{0,0}^{-1} u=B_{0,-1}^{-1} .
$$

Suponhamos por indução que a fórmula do item 1 é verdadeira para $r \leq-1$ e $s=0$ e vamos mostrar que a fórmula é verdadeira para $r-1$. O símbolo $*$ significa que estamos usando a hipótese de indução.

$$
\begin{aligned}
\Gamma_{0, r-1} & =u^{r-1} v u^{r-1} v^{-1}=u^{-1} u^{r} v u^{r}\left(v^{-1} u u^{-1} v\right) u^{-1} v^{-1}\left(u^{-1} u\right) \\
& =u^{-1}\left(u^{r} v u^{r} v^{-1}\right) u u^{-1}\left(u v u v^{-1}\right)^{-1} u \\
& =u^{-1} \Gamma_{0, r} u B_{0,-1}^{-1} \stackrel{*}{=} u^{-1}\left(\prod_{i=0}^{-r-1} B_{0, r+i}^{-1}\right) u B_{0,-1}^{-1} \\
& =\left(\prod_{i=0}^{-r-1} B_{0, r-1+i}^{-1}\right) B_{0,-1}^{-1}=\prod_{i=0}^{-(r-1)-1} B_{0,(r-1)+i}^{-1} .
\end{aligned}
$$

O caso geral é obtido do anterior notando que $\Gamma_{s, r}=v^{s} \Gamma_{0, r} v^{-s}$ e $B_{s, r}=v^{s} B_{0, r} v^{-s}$. A fórmula 2 . $B_{s, r}=\Gamma_{s, r+1} \Gamma_{s, r}^{-1}$ é de verificação direta e por isso omitimos os cálculos. 
Teorema B.0.21. O conjunto $\left\{B_{s, r}=v^{s} u^{r} B u^{-r} v^{-s}\right\}_{s, r \in \mathbb{Z}}$ é uma base de $\operatorname{ker} g$.

Demonstração. Notemos que pelo Lema B.0.20, o conjunto $\left\{B_{s, r}\right\}$ gera ker $g$. Para mostrar que este conjunto de fato é uma base, basta mostrar que só existem relações triviais entre esses geradores. Vamos fazer a prova por contradição. Suponhamos que exista uma palavra

$$
w=B_{s_{1}, r_{1}}^{\epsilon_{1}} B_{s_{2}, r_{2}}^{\epsilon_{2}} \cdots B_{s_{k}, r_{k}}^{\epsilon_{k}}=\mathbb{1}, \operatorname{com} \epsilon_{i} \in\{-1,1\} \text { e } B_{s_{i}, r_{i}}^{\epsilon_{i}} B_{s_{i+1}, r_{i+1}}^{\epsilon_{i+1}} \neq 1
$$

Seja $S$ o conjunto dos índices $\left\{s_{1}, s_{2}, \ldots, s_{k}\right\}$. Fixemos $s \in S$. Seja $R_{s}$ o conjunto dos índices $r_{j}$ tal que o gerador $B_{s, r_{j}}$ aparece na palavra $w$. Sejam $r_{m}$ e $r_{M}$ o elemento minimal e o elemento maximal de $R_{s}$, respectivamente. Definimos os subconjuntos $B_{s}$ e $\Gamma_{s}$ de acordo com as seguintes possibilidades:

- $B_{s}=\left\{B_{s, 0}, \ldots, B_{s, r_{m}}, \ldots, B_{s, r_{M}}\right\}$ e $\Gamma_{s}=\left\{\Gamma_{s, 1} \ldots, \Gamma_{s, r_{M}}, \Gamma_{s, r_{M}+1}\right\}$, se $0 \leq r_{m}$

- $B_{s}=\left\{B_{s, r_{m}}, \ldots, B_{s,-1}, B_{s, 0}, B_{s, 1}, \ldots, B_{s, r_{M}}\right\}$ e $\Gamma_{s}=\left\{\Gamma_{s, r_{m}}, \ldots, \Gamma_{s,-1}, \Gamma_{s, 1}, \ldots, \Gamma_{s, r_{M}+1}\right\}$, se $r_{m}<0<r_{M}+1$

- $B_{s}=\left\{B_{s, r_{m}}, \ldots, B_{s, r_{M}}\right\}$ e $\Gamma_{s}=\left\{\Gamma_{s, r_{m}}, \ldots, \Gamma_{s, r_{M}}\right\}$, se $r_{M}+1=0$

- $B_{s}=\left\{B_{s, r_{m}}, \ldots, B_{s, r_{M}}, B_{s, r_{M}+1}\right\}$ e $\Gamma_{s}=\left\{\Gamma_{s, r_{m}}, \ldots, \Gamma_{s, r_{M}}, \Gamma_{s, r_{M}+1}\right\}$, se $r_{M}+1<0$

Notemos que em todos os casos, $B_{s}$ e $\Gamma_{s}$ tem a mesma cardinalidade e ambos geram um mesmo subgrupo de ker $g$, de acordo com o Lema B.0.20.

Agora notemos que se $s, s^{\prime} \in S$, com $s \neq s^{\prime}$, então $B_{s} \cap B_{s^{\prime}}=\emptyset=\Gamma_{s} \cap \Gamma_{s^{\prime}}$. Assim, temos que os conjuntos $C_{B}=\bigcup_{s \in S} B_{s}$ e $C_{\Gamma}=\bigcup_{s \in S} \Gamma_{s}$ tem a mesma cardinalidade finita, e geram um mesmo subgrupo $C$. Como $C_{\Gamma}$ é base de $C$, segue $C_{B}$ também o é. Notemos que a palavra $w$ está escrita em termos dos geradores $C_{B}$, o que é um absurdo, pois $w=\mathbb{1}$. Segue a conclusão. 
124 APÊNDICE B 


\section{Apêndice C}

\section{O grupo fundamental dos espaços de configuração $\mathcal{A}_{2}\left(\mathbb{S}^{2}\right)$ e $\mathcal{B}_{2}\left(\mathbb{S}^{2}\right)$} fixos

Seja $\mathcal{A}_{2}\left(\mathbb{S}^{2}\right)=\left\{(x, y) \in \mathbb{S}^{2} \times \mathbb{S}^{2} \mid x \neq \pm y\right\}$. Neste espaço, definimos a involução livre de pontos

$$
\begin{aligned}
\tau_{1}: \mathcal{A}_{2}\left(\mathbb{S}^{2}\right) & \longrightarrow \mathcal{A}_{2}\left(\mathbb{S}^{2}\right) \\
(x, y) & \longmapsto(y, x)
\end{aligned}
$$

e denotamos por $\mathcal{B}_{2}\left(\mathbb{S}^{2}\right)$ o espaço de órbitas.

O objetivo deste apêndice é mostrar que $\pi_{1}\left(\mathcal{A}_{2}\left(\mathbb{S}^{2}\right)\right)$ e $\pi_{1}\left(\mathcal{B}_{2}\left(\mathbb{S}^{2}\right)\right)$ são cíclicos de ordem 2 e 4 , respectivamente. Consideremos os elementos $B \in \pi_{1}\left(\mathcal{A}_{2}\left(\mathbb{S}^{2}\right)\right)$ e $\sigma \in \pi_{1}\left(\mathcal{B}_{2}\left(\mathbb{S}^{2}\right)\right)$, que são as classes de homotopia dos laços representados nas Figuras C.1 e C.2, respectivamente. Mais especificamente, mostraremos que $\pi_{1}\left(\mathcal{A}_{2}\left(\mathbb{S}^{2}\right)\right)=\left\langle B \mid B^{2}=1\right\rangle$ e $\pi_{1}\left(\mathcal{B}_{2}\left(\mathbb{S}^{2}\right)\right)=\left\langle\sigma \mid \sigma^{4}=1\right\rangle$.

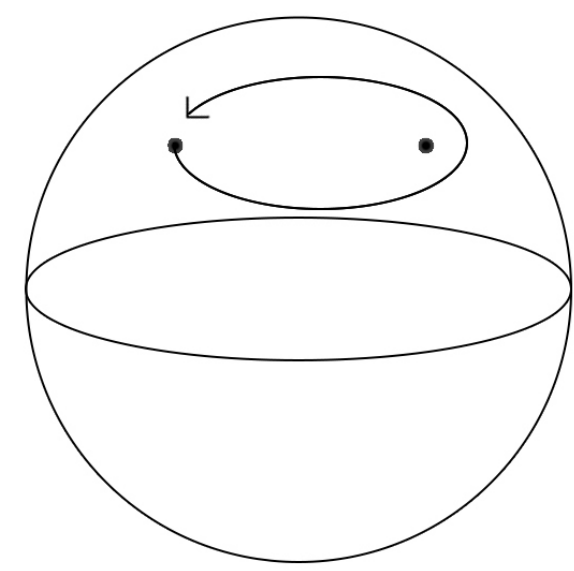

Figura C.1: $B \in \pi_{1}\left(\mathcal{A}_{2}\left(\mathbb{S}^{2}\right)\right)$.

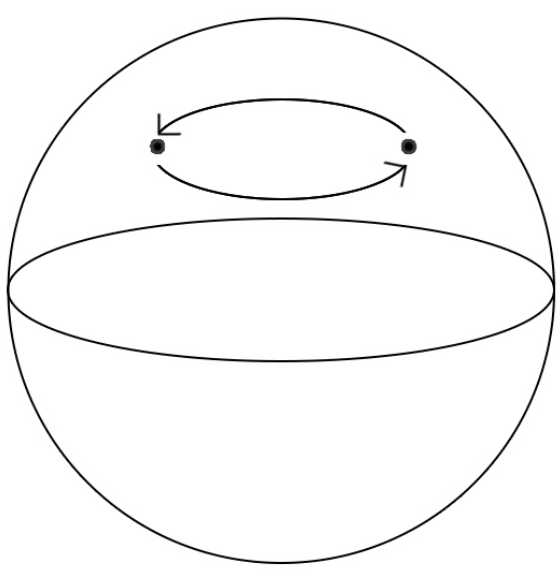

Figura C.2: $\sigma \in \pi_{1}\left(\mathcal{B}_{2}\left(\mathbb{S}^{2}\right)\right)$.

Seja $p_{A}: \mathbb{S}^{2} \rightarrow \mathbb{R P}^{2}$ o recobrimento duplo induzido da função antipodal $A: \mathbb{S}^{2} \rightarrow \mathbb{S}^{2}$, e seja $p=p_{A} \times p_{A}: \mathbb{S}^{2} \times \mathbb{S}^{2} \rightarrow \mathbb{R P}^{2} \times \mathbb{R P}^{2}$ o recobrimento produto.

Notemos que

$$
F_{2}\left(\mathbb{R} \mathbb{P}^{2}\right)=\mathbb{R} \mathbb{P}^{2} \times \mathbb{R P}^{2}-\Delta \text { e } p^{-1}\left(F_{2}\left(\mathbb{R} \mathbb{P}^{2}\right)\right)=\mathcal{A}_{2}\left(\mathbb{S}^{2}\right) .
$$

sendo $\Delta=\left\{(x, x) \mid x \in \mathbb{R} \mathbb{P}^{2}\right\}$ e $F_{2}\left(\mathbb{R P}^{2}\right)$ como definido na Seção 1.4.

Por restrição, nós temos que $j=\left.p\right|_{\mathcal{A}_{2}\left(\mathbb{S}^{2}\right)}: \mathcal{A}_{2}\left(\mathbb{S}^{2}\right) \rightarrow F_{2}\left(\mathbb{R P}^{2}\right)$ é uma aplicação de recobrimento. Logo, é injetor o homomorfismo

$$
j_{\#}: \pi_{1}\left(\mathcal{A}_{2}\left(\mathbb{S}^{2}\right)\right) \rightarrow P_{2}\left(\mathbb{R} \mathbb{P}^{2}\right)=\pi_{1}\left(F_{2}\left(\mathbb{R} \mathbb{P}^{2}\right)\right) .
$$

Em [6, Seção VI], está demonstrado que $j_{\#}(B)$ é não trivial, e portanto $B \in \pi_{1}\left(\mathcal{A}_{2}\left(\mathbb{S}^{2}\right)\right)$ é não 
trivial.

Consideremos a variedade de Stiefel $V_{3,2}=\left\{\left(x_{1}, x_{2}\right) \in \mathbb{R}^{3} \times \mathbb{R}^{3} \mid\left\langle x_{i}, x_{j}\right\rangle=\delta_{i j}\right\}$, sendo $\delta_{i j}$ a função delta de Kronecker.

Em [11, Teorema 2.4], está demonstrado que $\mathcal{A}_{2}\left(\mathbb{S}^{2}\right)$ tem o mesmo tipo de homotopia que $V_{3,2}$. Em [27, Capítulo IV, Teorema 10.13], está demonstrado que $\pi_{1}\left(V_{3,2}\right)$ é isomorfo a $\mathbb{Z}_{2}$. Portanto, $\pi_{1}\left(\mathcal{A}_{2}\left(\mathbb{S}^{2}\right)\right)$ é cíclico de ordem 2 e como $B$ é não trivial, então

$$
\pi_{1}\left(\mathcal{A}_{2}\left(\mathbb{S}^{2}\right)\right)=\left\langle B \mid B^{2}=1\right\rangle .
$$

Tal qual nós fizemos na Seção 1.3, nós temos a seguinte sequência exata:

$$
1 \longrightarrow \pi_{1}\left(\mathcal{A}_{2}\left(\mathbb{S}^{2}\right)\right)=\left\langle B \mid B^{2}=1\right\rangle \stackrel{\left(p_{\tau_{1}}\right)_{\#}}{\longrightarrow} \pi_{1}\left(\mathcal{B}_{2}\left(\mathbb{S}^{2}\right)\right) \stackrel{\theta_{\tau_{1}}}{\longrightarrow} \mathbb{Z}_{2} \longrightarrow 1 .
$$

Logo, nós temos que ou $\pi_{1}\left(\mathcal{B}_{2}\left(\mathbb{S}^{2}\right)\right)$ é isomorfo a $\mathbb{Z}_{4}$ ou a $\mathbb{Z}_{2} \oplus \mathbb{Z}_{2}$. Geometricamente, é fácil ver que $\left(p_{\tau_{1}}\right)_{\#}(B)=\sigma^{2}$ e como $B$ é o gerador de $\pi_{1}\left(\mathcal{A}_{2}\left(\mathbb{S}^{2}\right)\right)$, então $\sigma^{2}$ não é o elemento trivial de $\pi_{1}\left(\mathcal{B}_{2}\left(\mathbb{S}^{2}\right)\right)$. Logo, $\pi_{1}\left(\mathcal{B}_{2}\left(\mathbb{S}^{2}\right)\right)$ é isomorfo a $\mathbb{Z}_{4}$ e $\sigma$ é o gerador.

Nós podemos resumir os fatos provados neste apêndice no seguinte resultado:

Teorema C.0.22. Os grupos $\pi_{1}\left(\mathcal{A}_{2}\left(\mathbb{S}^{2}\right)\right)$ e $\pi_{1}\left(\mathcal{B}_{2}\left(\mathbb{S}^{2}\right)\right)$ são cíclicos de ordem 2 e 4 , respectivamente, e temos a seguinte sequência exata

$$
1 \longrightarrow \pi_{1}\left(\mathcal{A}_{2}\left(\mathbb{S}^{2}\right)\right)=\left\langle B \mid B^{2}=1\right\rangle \stackrel{\left(p_{\tau_{1}}\right)_{\#}}{\longrightarrow} \pi_{1}\left(\mathcal{B}_{2}\left(\mathbb{S}^{2}\right)\right)=\left\langle\sigma \mid \sigma^{4}=1\right\rangle \stackrel{\theta_{\tau_{1}}}{\longrightarrow} \mathbb{Z}_{2} \longrightarrow 1
$$

sendo $\left(p_{\tau_{1}}\right)_{\#}(B)=\sigma^{2}$ e $\theta_{\tau_{1}}(\sigma)=\overline{1}$. 


\section{Referências Bibliográficas}

[1] M. A. Armstrong, Basic Topology, Undergraduate Texts in Mathematics, Springer - Verlag, (1983). 91

[2] E. Artin, Theorie der Zöpfe, Abhandlungen aus dem Mathematischen Seminar der Universität Hamburg 4 (1925), 47-72. 3

[3] P. Bellingeri, On presentations of surface braid groups, Journal of Algebra 274 (2004) 543-563. 105

[4] J. S. Birman, Braids, Links, and Mapping Class Groups, Annals of Mathematics Studies 82, Princeton University Press, (1974). 13, 15

[5] K. Borsuk, Drei Sätze über die n-dimensionale Euklidische Sphäre, Fundamenta Mathematicae 20 (1933) 177-190. 1

[6] J. V. Buskirk, Braid Groups of Compact 2-Manifolds with Elements of Finite Order, Transactions of the American Mathematical Society $122 \mathrm{n}^{0} 1$ (1966) 81-97. 125

[7] P. E. Conner, E. E. Floyd, Differentiable periodic maps, Springer - Verlag, (1964).

[8] T. tom Dieck, Transformation Groups, de Gruyter studies in mathematics 8, (1987). 87, 94

[9] A. Dold, Lectures on Algebraic Topology, Springer - Verlag, (1972). 86

[10] D. B. A. Epstein, The degree of a map, Proccedings of the London Mathematical Society s3-16 (1966) 369-383. 82

[11] E. Fadell, Homotopy groups of configuration spaces and the string problem of Dirac, Duke Mathematical Journal 29 (1962) 231-242. 126

[12] E. Fadell, S. Husseini, The Nielsen number on surfaces, Contemporary Mathematics 21 (1983) 59-99. 99

[13] E. Fadell, L Neuwirth, Configuration Spaces, Mathematica Scandinavica 10 (1962) 111-118. 13,96

[14] R. H. Fox, L. Neuwirth, The braid groups, Mathematica Scandinavica 10 (1962), 119-126. 3

[15] D. L. Gonçalves, The Borsuk-Ulam theorem for surfaces, Quaestiones Mathematicae 29 (2006), no. $1,117-123.1,2,5,7,8$

[16] D. L. Gonçalves, J. Guaschi, The Borsuk-Ulam for maps into a surface, Topology and its Applications 157 (2010) 1742 - 1759. 1, 2, 5, 7, 8

[17] D. L. Gonçalves, J. Guaschi, The fixed points of multimaps on surface with application to the torus - a Braid approach, preprint. 99

[18] D. L. Gonçalves, M. R. Kelly, Wecken type problems for self-maps of the Klein bottle, Fixed Point Theory and Applications (2006), 1 -15. 33 
[19] D. L. Gonçalves, M. R. Kelly, Coincidence Properties for Maps from the Torus to the Klein Bottle, Chinese Annals of Mathematics 29B (2008), 425-440. 39

[20] V. L. Hansen, Braids and Coverings: selectd topics, London Mathematical Society Student Texts 18, Cambridge University. Press, (1989). 13

[21] W. Magnus, A. Karras, D. Solitar, Combinatorial Group Theory: Presentations of groups in terms of generators and relations, Interscience Publishers, 1966. 106

[22] W. S. Massey, Algebraic Topology: An Introduction, Graduate Texts in Mathematics 56, Springer - Verlag, (1967). 105

[23] J. Matoušek, Using the Borsuk-Ulam Theorem, Universitext, Springer - Verlag, (2002). 1

[24] K. Murasugi, B. I. Kurpita, A Study of Braids, Mathematics and Its Aplications 484, Kluwer Academic Publishers, (1999). 13, 106

[25] G. P. Scott, Braid groups and the group of homeomorphisms of a surface, Mathematical Proceedings of the Cambridge Philosophical Society 68 (1970), 605-617. 99

[26] J. W. Vick, Homology Theory: An Introduction to Algebraic Topology, Graduate Texts in Mathematics 145, Springer - Verlag, (1994). 16

[27] G. W. Whitehead, Elements of homotopy theory, Graduate Texts in Mathematics 61, Springer - Verlag, (1978). 6, 11, 79, 126 